\title{
Extracellular matrix derived peptides and mesenchymal stem cell motility
}

Sandi Grainne Dempsey

School of Biological Sciences

Department of Science

Victoria University of Wellington

This dissertation is submitted for the degree of Doctor of Philosophy September 2016 


\section{Declaration}

This dissertation is the result of my own work and includes nothing which is the outcome of work done in collaboration except where specifically indicated in the text. It has not been previously submitted, in part or whole, to any university or institution for any degree, diploma, or other qualification.

In accordance with the departmental guidelines, this thesis is does not exceed 100,000 words, and it contains less than 150 figures.

Signed:

Date:

Sandi Grainne Dempsey 


\begin{abstract}
Biomaterials derived from decellularised extracellular matrices have shown promise as tools in tissue regeneration and wound healing. Such materials display biocompatibility as well as inherent bioactivity, promoting constructive remodelling in healing tissues. In this study, the bioactivity of ovine forestomach matrix (a decellularised extracellular matrix biomaterial) is assessed based on its ability to affect the proliferation and migration of wound healing cells.

This material supported cell attachment and proliferation, but did not allow cell infiltration in vitro. Enzymatic digestion of the material rendered soluble components that were able to induce proliferation and migration of some cell types. Cell-mediated processing of the material generated a protein or proteins with chemotactic activity for mesenchymal stem cells in vitro. Mass spectrometry analysis indicated the bioactive component consisted of the proteoglycan decorin, or fragments thereof.

Decorin has not previously been shown to induce mesenchymal stem cell motility, and these findings may add to what is known about decorin and its role in constructive remodelling. Furthermore, this cell-mediated approach for ECM breakdown could lead to the discovery of other bioactive peptides involved in ECM remodelling and wound healing.
\end{abstract}




\section{Acknowledgements}

I'd like to acknowledge my supervisors, Dr Darren Day and Dr Barnaby May. You have shared your support, patience and ingenuity and I have learned so much from you both. Thank you for the drive to write something I am proud of.

A number of scientists at Victoria University, Callaghan Innovation and Aroa Biosurgery have made their time and expertise available to me during this project. I'd like to extend my gratitude to Dr Ryan Steel, Dr Jonathan Dunne, Dr Leonardo Negron, Stanley Lun, Dr Nathaniel Dasyam, Danyl McLauchlan, Derek Heath and Sushila Pillai. Essential cell lines in this work were isolated by Dr Jun Jia at VUW and Dr Sarah Gunningham at Otago University in Christchurch. I'd also like to acknowledge Matthew Fitzhenry, Xiaomin Song and your team at the Australian Proteome Analysis Facility; you went out of your way to help me problem solve. Thank you Dr Shahista Nisa and Dr Susan Burgess for your editing skills.

I am eternally grateful to my amazing friends and canine walking companions, you literally kept me going. Thanks for the moral support and free pats; Dave, Kailey, Ian F, Marianne, James, Christian, Sha, Katy, Na, Luke, Otis, Amanda, Paulie, Varun, Zlatka, Josh, Jeremy O, Ian B, Alana, Soph, Del, Matt W, Miri, Pat, Blue, Patch, Pepper, Rosa, Rufus, Nala, Charlie, Naevey, Bo, Rocket and Rua.

Dr Christopher Miller, I couldn't decide which section to put you because you have been an honorary supervisor, peer and friend all at once. So you get your own section. Thanks buddy.

Finally, my beautiful growing whanau; Mum, Dad, Rachel, Geoff, Sophia, Emily, Rufus, Candice, Ani, Paddy, Liz. Thank you for your love, support and patience. 


\section{Contents}

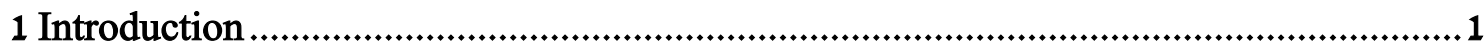

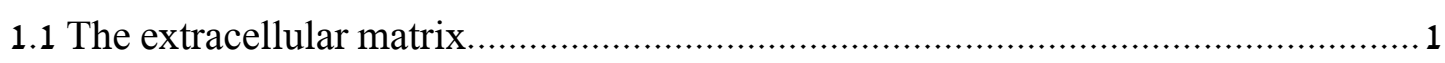

1.1.1 The composition of the extracellular matrix ...............................................

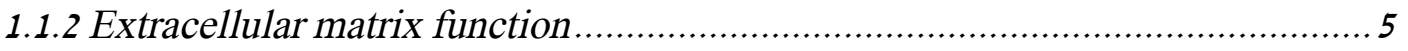

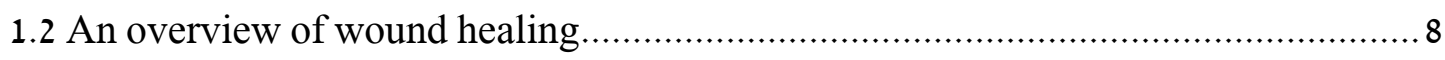

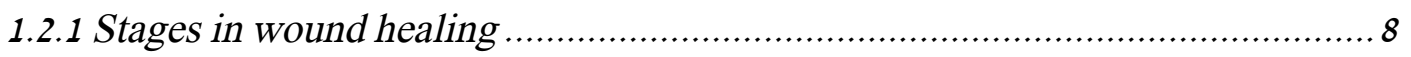

1.3 Important contributors to the wound healing process..................................... 10

1.3.1 Extracellular matrix components in wound healing ................................ 10

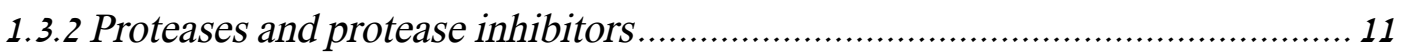

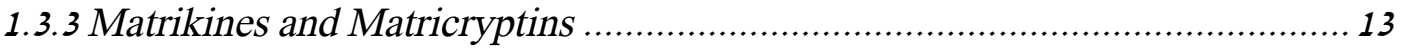

1.3.4 Cells, cytokines, chemokines and growth factors in wound healing .............. 14

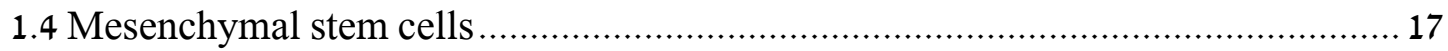

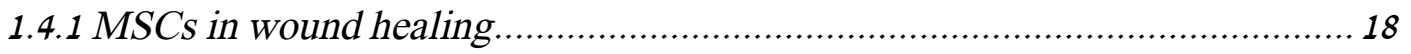

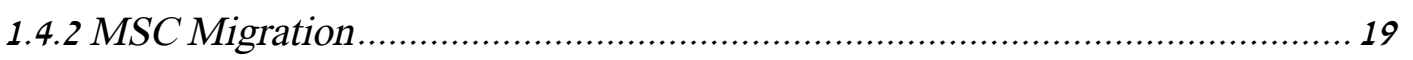

1.5 Wound healing, scar formation and tissue regeneration..................................... 20

1.5.1 The importance of the immune response during healing ........................... 21

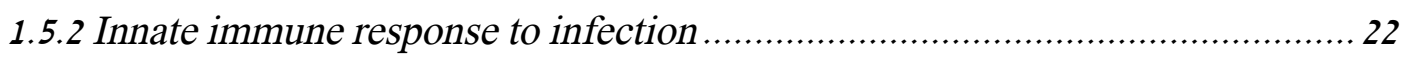

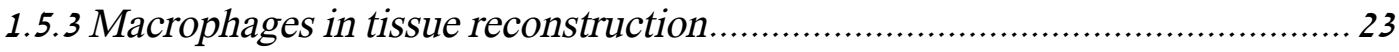

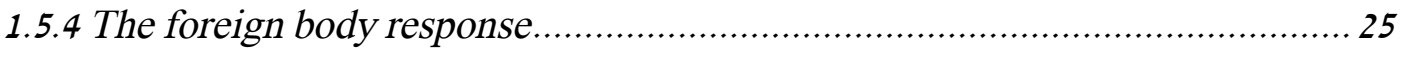

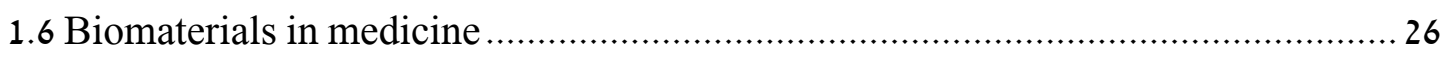

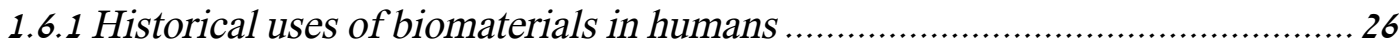

1.6.2 The scope of modern day biomaterials ................................................2

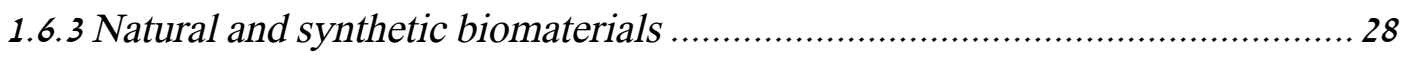

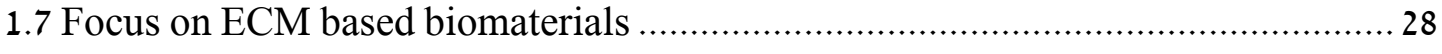

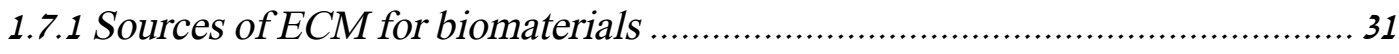

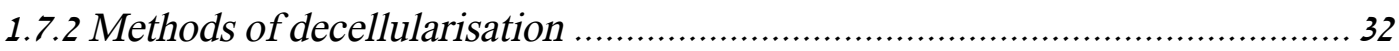

1.7.3 The behaviour of ECM materials during tissue regeneration ....................... 35

1.7.4 Evidence of bioactive moieties in dECM biomaterials .............................. 37

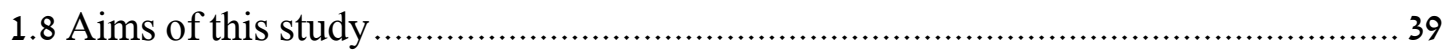




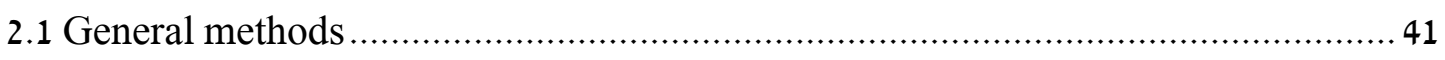

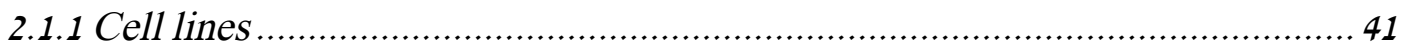

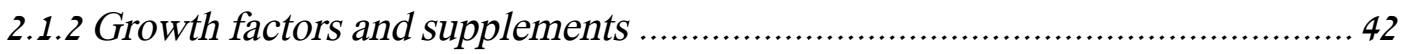

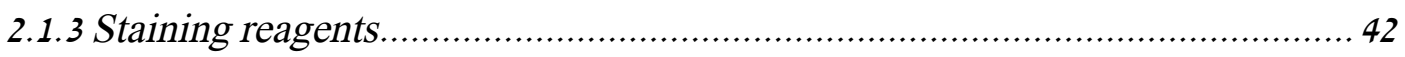

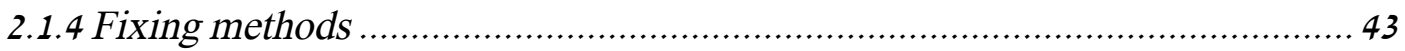

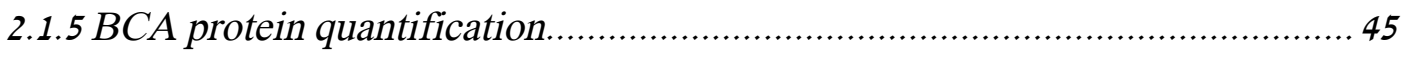

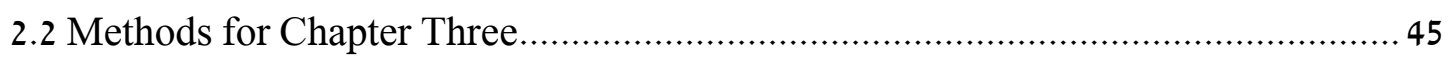

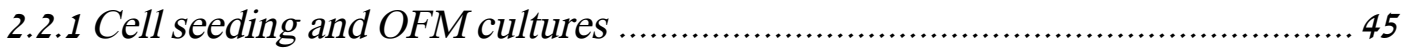

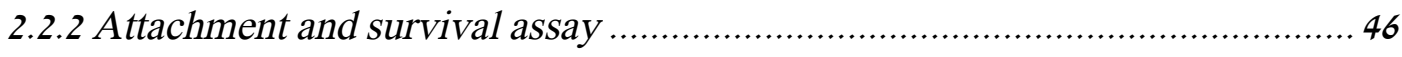

2.2.3 MSC and FB proliferation and infiltration of OFM............................... 47

2.2.4 Automated image processing and data analysis..................................... 48

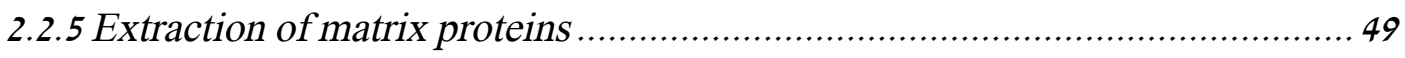

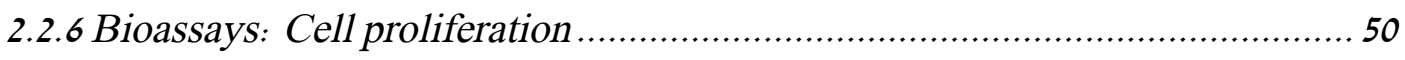

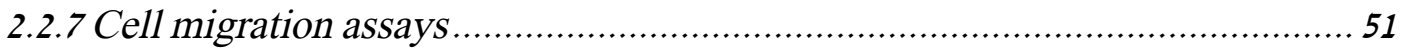

2.2.8 Preparation of dECM material from other tissue ..................................... 54

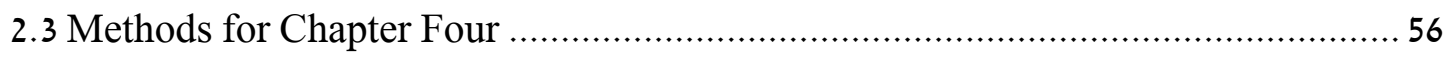

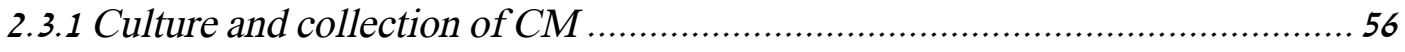

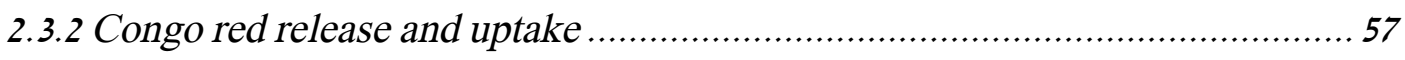

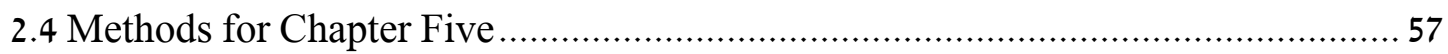

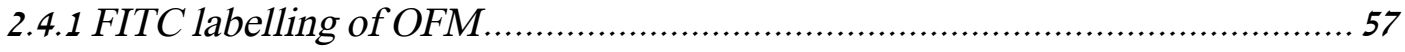

2.4.2 Scale up of CM sample collection ..................................................... 58

2.4.3 Imaging of macrophages after CM collection ...........................................58

2.4.4 Separation of macrophage and OFM derived proteins by SDS-PAGE ..........59

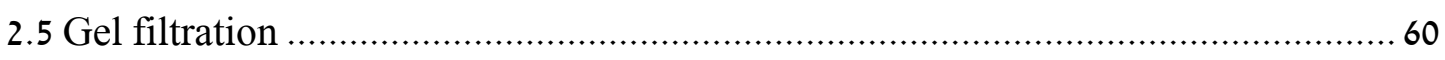

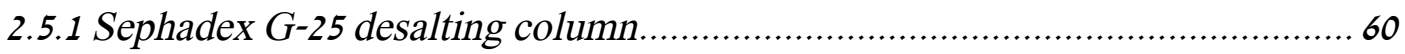

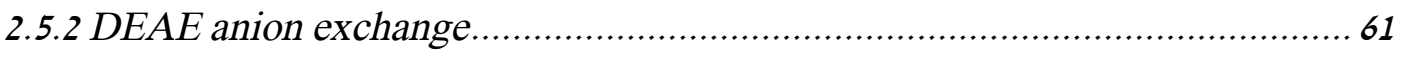

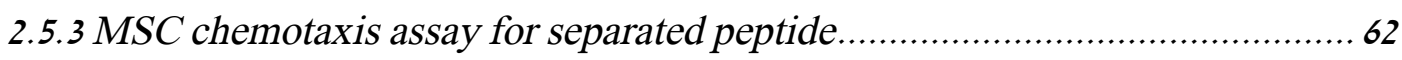

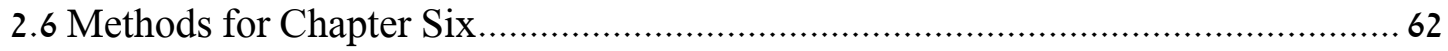

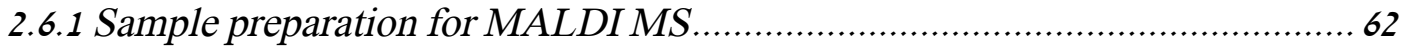




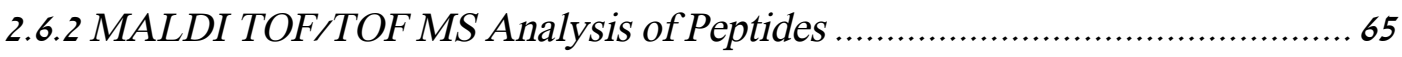

2.6.3 Protein matching with Protein Pilot and MASCOT ….................................65

2.6.4 Sample preparation for MALDI and ESI at APAF .................................66

2.6.5 Theoretical digestion of protein candidate using MEROPS database ............. 66

3 Dynamic reciprocity between wound healing cells and the extracellular matrix ...........67

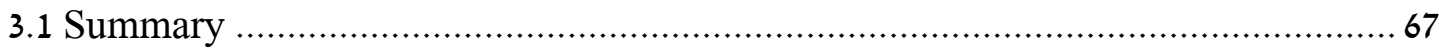

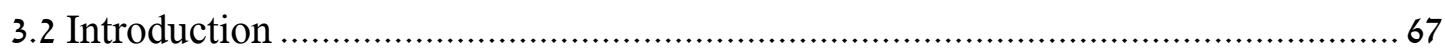

3.2.1 Modelling an ECM culture of wound healing cells in vitro..........................68

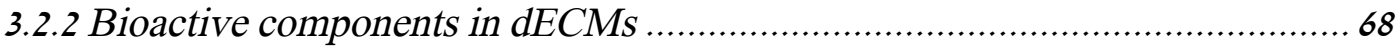

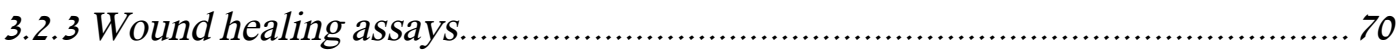

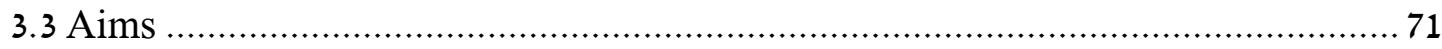

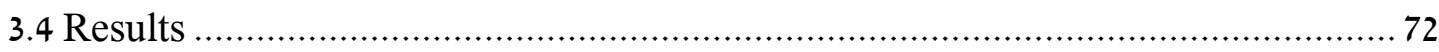

3.4.1 Cell attachment on OFM in vitro .............................................................. 72

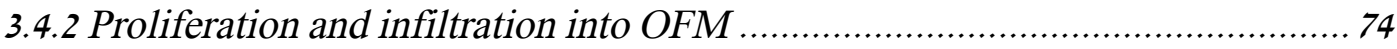

3.4.3 Bioactivity of OFM proteins in solution: cell proliferation.......................... 76

3.4.4 Bioactivity of OFM proteins in solution: cell migration ............................ 78

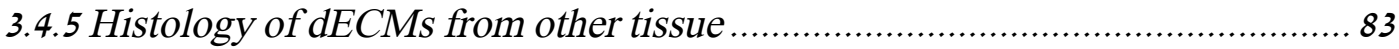

3.4.6 Comparison of bioactivity in different ECM tissue ................................... 84

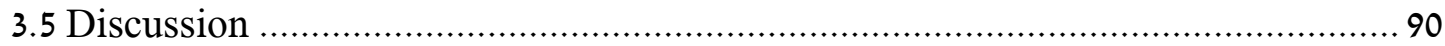

3.5.1 Attachment, proliferation and infiltration of OFM by cells .........................90

3.5.2 The use of OFM as a three dimensional cell culture substrate...................... 92

3.5.3 Bioactivity of soluble and pepsin digested components of OFM .................. 95

3.5.4 Bioactivity of dECMs derived from different source tissue ........................97

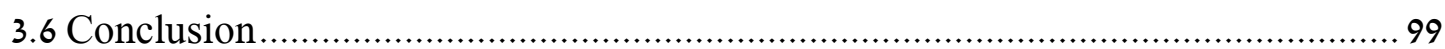

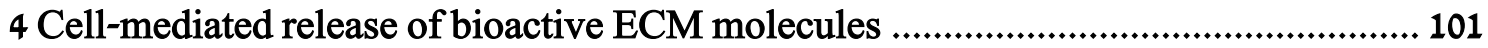

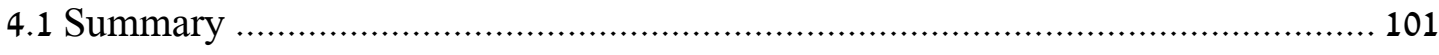

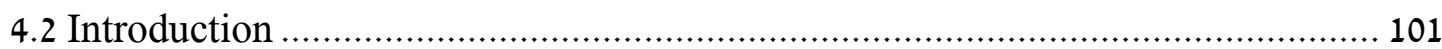

4.2.1 Protease expression by cells in wound healing.................................... 102

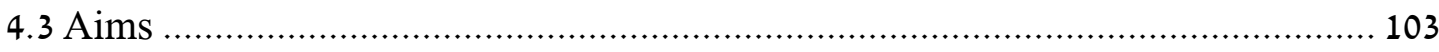

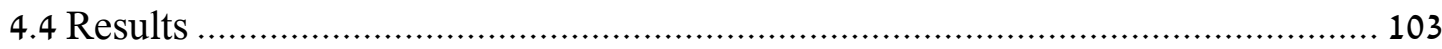




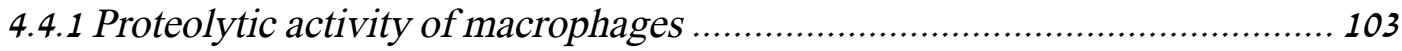

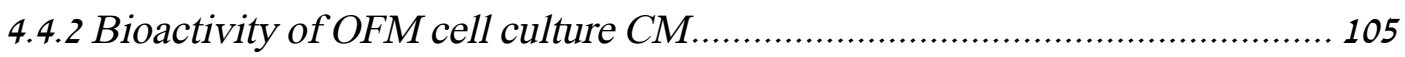

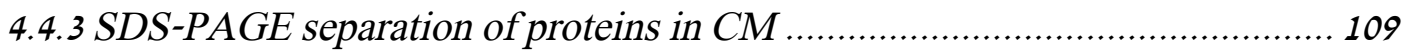

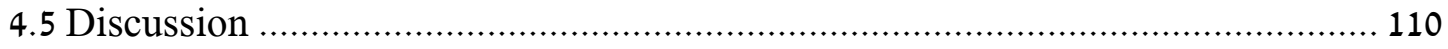

4.5.1 Macrophage-mediated processing of OFM ....................................... 110

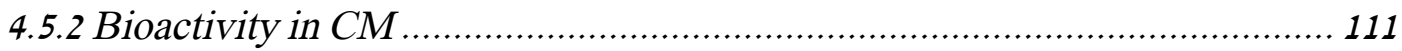

4.5.3 Macrophages grown on OFM recruit MSCs in vitro ............................. 111

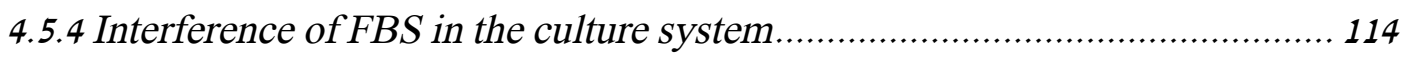

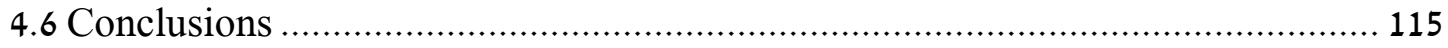

5 Macrophage-mediated release of an ECM derived protein ...................................... 117

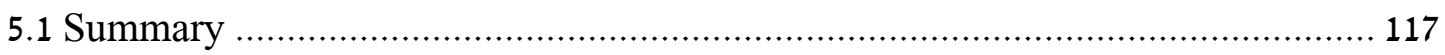

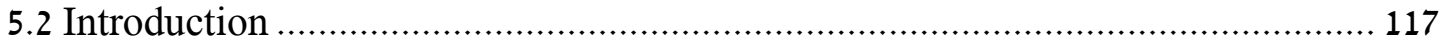

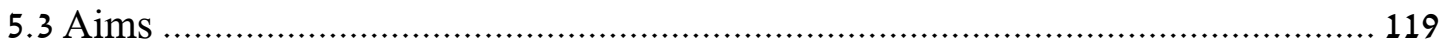

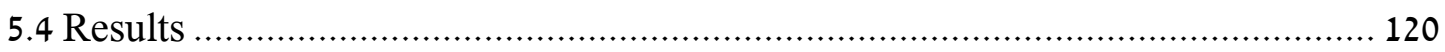

5.4.1 Macrophage uptake of FITC labelled protein ..................................... 120

5.4.2 SDS-PAGE analysis of FITC labelled protein....................................... 121

5.4.3 Improving resolution of SDS-PAGE separation.................................... 123

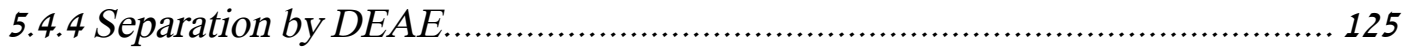

5.4.5 MSC chemotaxis using enriched peptide A........................................ 129

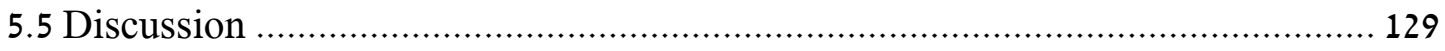

5.5.1 A distinct $12 \mathrm{kDa}$ OFM derived peptide may be cleaved by macrophages... 130

5.5.2 Enrichment of peptide $A$ by DEAE anion exchange ............................... 131

5.5.3 Bioactivity of peptide A in MSC migration assay .................................. 131

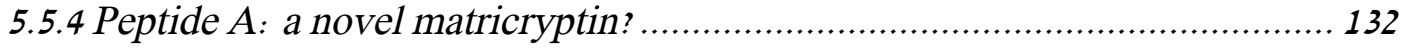

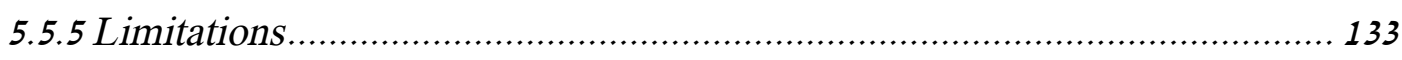

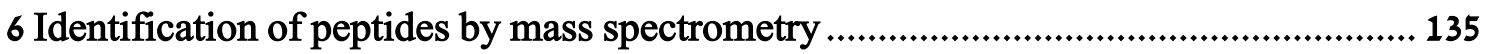

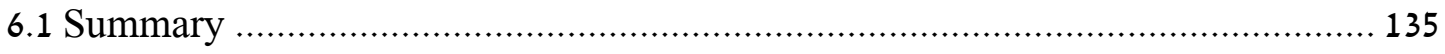

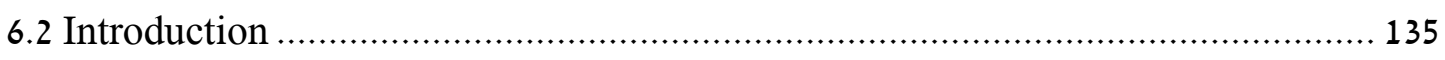

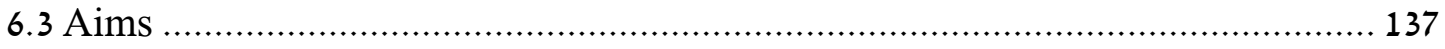

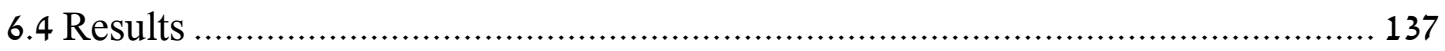


6.4.1 MALDI analysis of proteins in MO-CM sample.................................... 137

6.4.2 MALDI TOF/TOF MS analysis of gel band ...................................... 138

6.4.3 LC ESI MS/MS analysis of dried sample and gel band .......................... 141

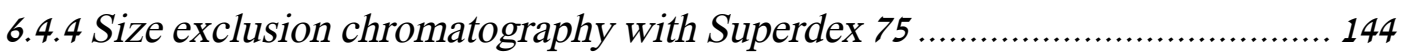

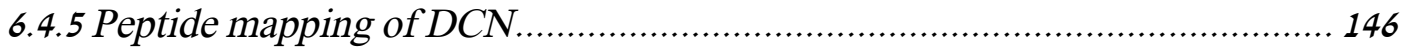

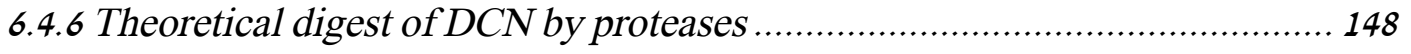

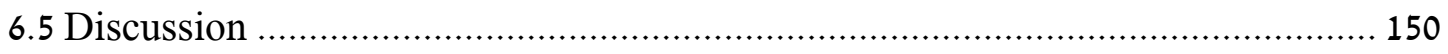

6.5.1 Evidence for macrophage processing of DCN...................................... 150

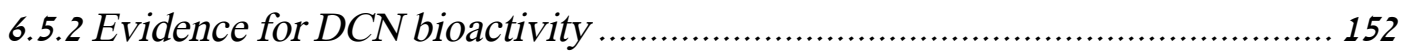

6.5.3 Potential use for protein as a mediator of cell migration ........................... 154

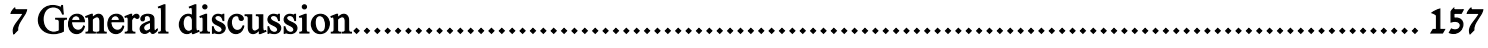

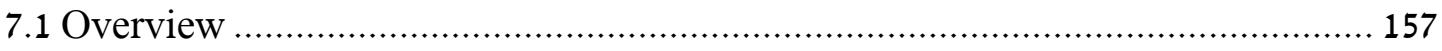

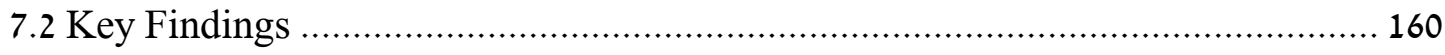

7.2.1 Proteolytic digestion of dECM biomaterials in wound healing ................... 160

7.2.2 Decorin as a cryptic peptide in biomaterial facilitated wound healing ......... 161

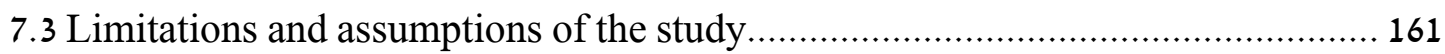

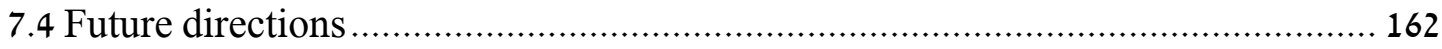

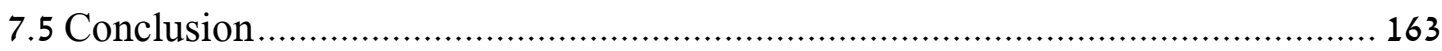

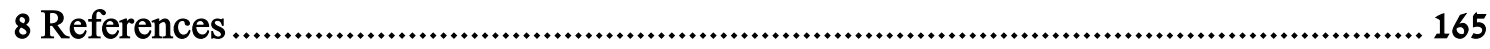

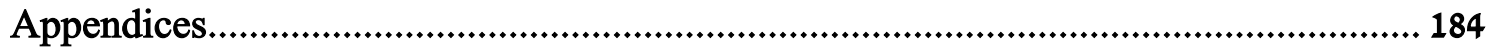

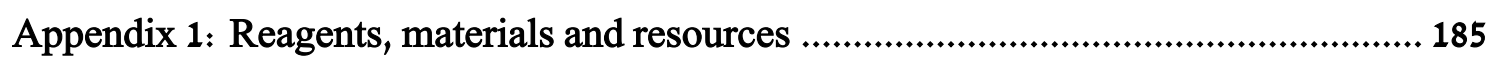

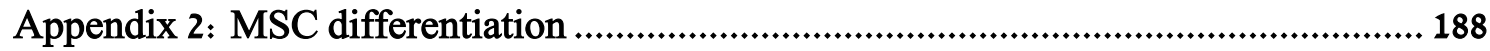

Appendix 3: Automated counting of cell-OFM culture cross sections ........................... 190

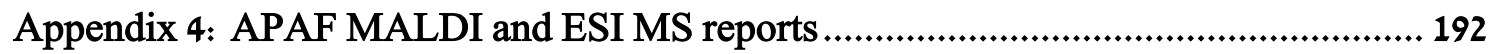

Appendix 5: A gradient based chemotaxis assay for mammalian cell motility .............. 209 


\section{List of Tables}

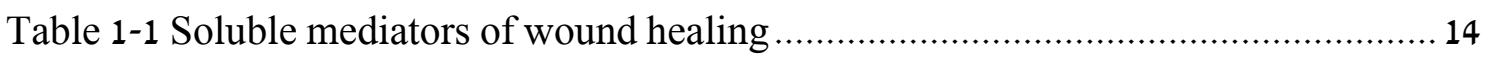

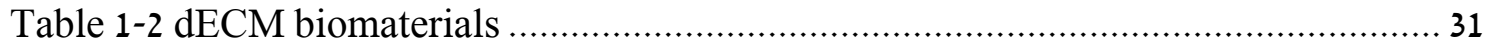

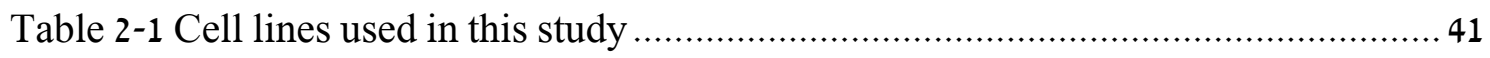

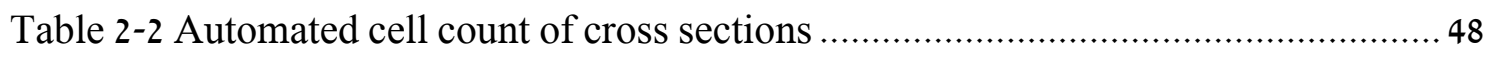

Table 2-3 Image J settings for automated counting of sections ........................................48

Table 3-1 Summary of proliferation activity of OFM extracts .................................... 78

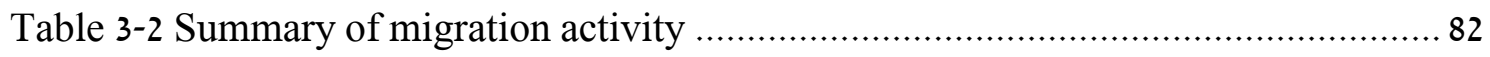

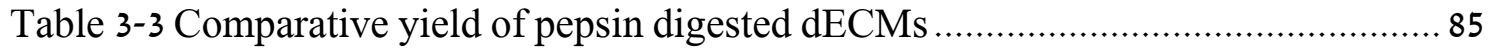

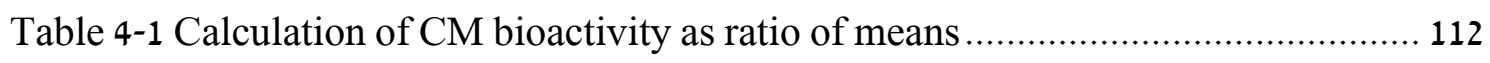

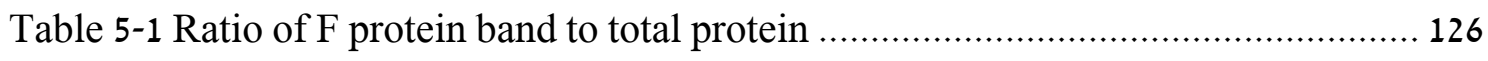

Table 6-1 MASCOT results for peptides generated by MALDI ................................ 138

Table 6-2 MASCOT protein matches of undiluted MO-CM ….................................. 142

Table 6-3 MASCOT protein matches of gel excised MO-CM................................... 143

Table 6-4 MASCOT matches of LCMS MO-CM with Ovis aries database. .................. 146

Table 6-5 Peptide matches of DCN protein using trypsin and GluC ............................ 147 


\section{List of Figures}

Figure 1-1 The extracellular matrix surrounding a cell .................................................

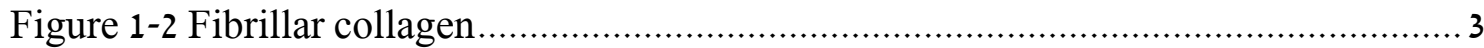

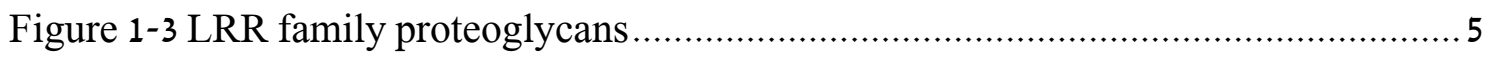

Figure 1-4 Integrins connect the cell to ECM proteins ...................................................6

Figure 1-5 Macrophage phenotypes in wound repair and inflammation........................ 24

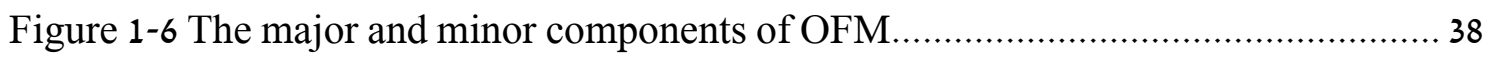

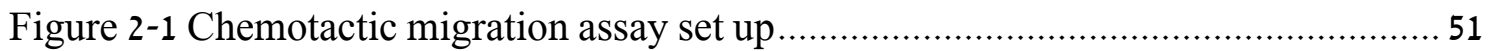

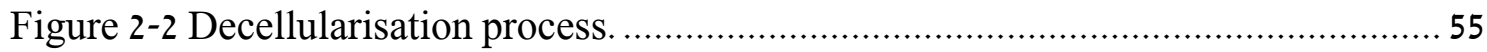

Figure 2-3 Collection of cell-CM, OFM-CM and cell-OFM-CM .................................... 56

Figure 3-1 FB attachment to conditioned discs of OFM in vitro ................................... 73

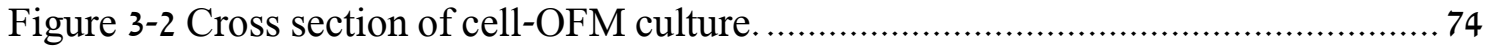

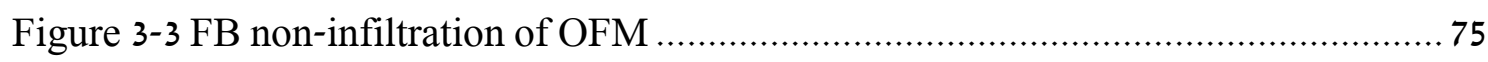

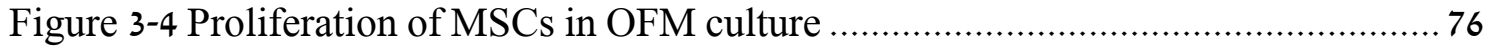

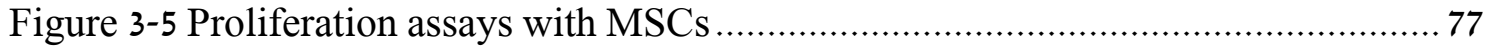

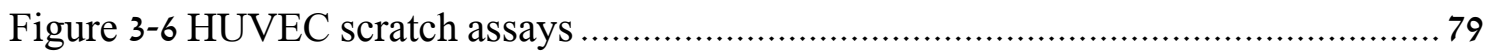

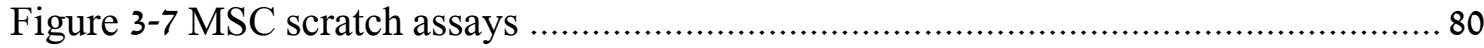

Figure 3-8 Chemotaxis assays with pepsin digested OFM........................................ 81

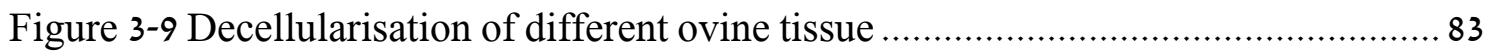

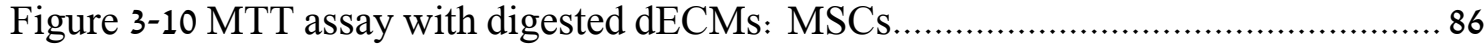

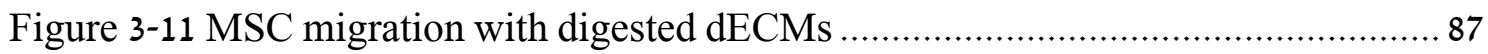

Figure 3-12 HUVEC migration with digested dECMs .................................................. 89

Figure 4-1 Congo red release by seeded macrophages.............................................. 104

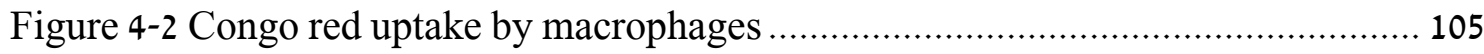

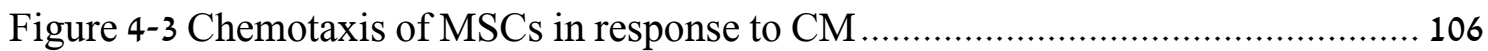

Figure 4-4 Chemotaxis of FBs in response to CM ................................................. 107

Figure 4-5 Chemotaxis of HUVECs in response to CM.............................................. 108

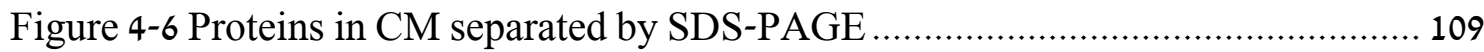




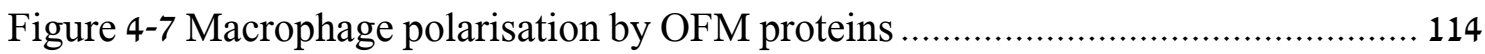

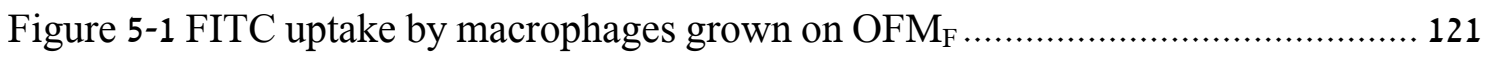

Figure 5-2 Labelled and unlabelled CM on 20 \% SDS-PAGE ..................................... 122

Figure 5-3 Labelled and unlabelled CM on $16 \%$ Tricine SDS-PAGE ......................... 124

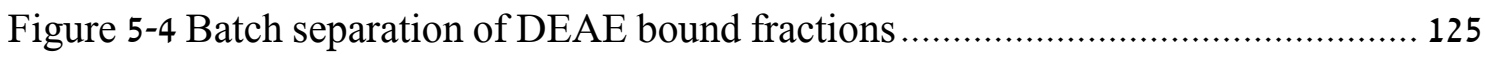

Figure 5-5 DEAE step gradient column separation of $\mathrm{MO}_{\mathrm{F}}$ sample............................ 127

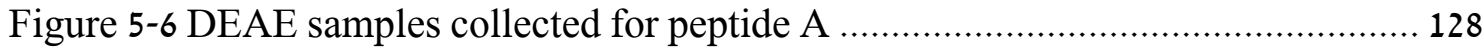

Figure 5-7 Chemotaxis of enriched fraction of peptide A …...................................... 129

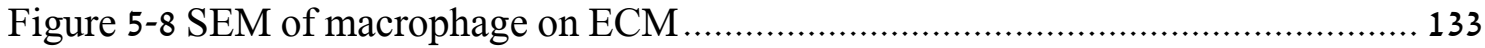

Figure 6-1 Gel extraction of proteins in the MO-CM sample ...................................... 137

Figure 6-2 MALDI spectra of enriched fraction of MO-CM 1D ............................... 139

Figure 6-3 MS spectrum of peptides generated by MALDI-TOF by APAF ................. 140

Figure 6-4 Size exclusion chromatogram MO-CM sample....................................... 145

Figure 6-5 The distribution of DCN derived peptides identified by ESI ....................... 148

Figure 6-6 DCN fragments generated from known proteolytic cleavage sites.............. 149

Figure 6-7 Phenotype associated MMP expression of macrophages ........................... 151

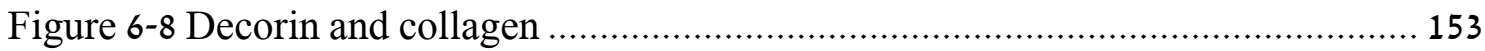




\section{List of Abbreviations and Acronyms}

$\mathrm{ACN} \quad$ acetonitrile

ADAM a disintegrin and metalloprotease

ADAMTS a disintegrin and metalloprotease with thrombospondin motifs

ATCC American Type Culture Collection

CAM chorioallantoic membrane

CCL chemokine (C-C motif) ligand

CCR chemokine (C-C motif) receptor

CHCA $\quad \alpha$-cyano-4-hydroxycinnamic acid

CM conditioned media

CXCR chemokine (C-X-C motif) receptor

D551 Detroit 551 (foetal dermal fibroblast cell line)

DAPI 4',6-diamidino-2-phenylindole

$\mathrm{ddH}_{2} \mathrm{O} \quad$ double distilled water

DCN decorin

DDR discoidin domain receptor

dECM decellularised extracellular matrix

DMEM Dulbecco's modified eagle medium

DMSO dimethyl sulfoxide

DNA deoxyribonucleic acid

DTT dithiothreitol

ECGS endothelial cell growth supplement

ECM extracellular matrix

EGF endothelial growth factor

ELISA enzyme-linked immunosorbent assay

EOMA endothelioma cell

ESI electrospray ionisation

FB fibroblast

FBS foetal bovine serum 


\begin{tabular}{|c|c|}
\hline FGF & fibroblast growth factor \\
\hline FITC & fluorescein isothiocyanate \\
\hline G-CSF & granulocyte-colony stimulating factor \\
\hline GAG & glycosaminoglycan \\
\hline HS & horse serum \\
\hline HSC & hematopoietic stem cell \\
\hline HUVEC & human umbilical vein endothelial cell \\
\hline IA & iodoacetamide \\
\hline IFN & interferon \\
\hline IL & interleukin \\
\hline LRR & leucine rich repeat \\
\hline MALDI & matrix-assisted laser desorption/ionisation \\
\hline MMP & matrix metalloprotease \\
\hline MS & mass spectrometry \\
\hline MSC & mesenchymal stem cell \\
\hline MSF & migration stimulating factor \\
\hline MT-MMP & membrane type matrix metalloprotease \\
\hline MTT & 3-(4,5-dimethylthiazol-2-yl)-2,5-diphenyltetrazolium bromide \\
\hline OCT & optimal cutting temperature \\
\hline OFM & ovine forestomach matrix \\
\hline PBS & phosphate buffered saline \\
\hline $\mathrm{PC} 12$ & pheochromocytoma cell line (from rat adrenal medulla) \\
\hline PDGF & platelet derived growth factor \\
\hline PMSF & phenylmethylsulfonyl fluoride \\
\hline rpm & revolutions per minute \\
\hline RPMI & Roswell Park Memorial Institute 1640 medium \\
\hline SDF1 & stromal cell derived factor 1 \\
\hline SDS & sodium dodecyl sulphate \\
\hline SIS & small intestinal submucosa \\
\hline TFA & trifluoroacetic acid \\
\hline
\end{tabular}


TGF

transforming growth factor

TIMP tissue inhibitor of matrix metalloprotease

TOF time of flight 


\section{List of Appendices}

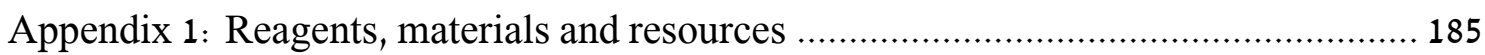

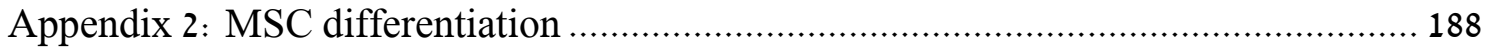

Appendix 3: Automated counting of cell-OFM culture cross sections ......................... 190

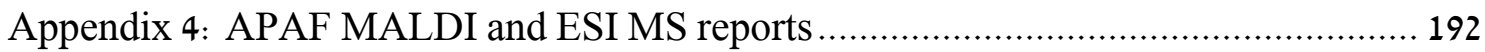

Appendix 5: A gradient based chemotaxis assay for mammalian cell motility ............ 209 



\section{Introduction}

In living tissue, the extracellular matrix (ECM) has many vital roles. Most cells require a supportive ECM, be they fat cells in adipose tissue or epithelial cells anchored to a basement membrane. The ECM modifies cell function, survival and homeostasis. Cells in turn are continually remodelling the arrangement of structural proteins and growth factors of their ECM. Extracellular matrix derived biomaterials are biochemically and structurally similar to native tissue, rendering them biocompatible and intrinsically bioactive. Evidence has suggested that these materials play an important role in the constructive remodelling of injured tissue ${ }^{1}$. As such, they can be used for tissue repair and regenerative applications. This study explores the relationship between such a material and mesenchymal stem cells, an important type of wound healing cell.

This chapter begins with a description of major ECM components and their functions in living tissue. Following this, an overview of the wound healing process is presented with an emphasis on ECM components and proteases that direct the cells in this process. A description of mesenchymal stem cells and their function in wound healing and tissue regeneration is given, before introducing the role of the immune system in constructive remodelling. The foreign body response to injury is descriped, linking wound healing to the field of biomaterials. A brief history of the field is given, with particular reference to ECM based biomaterials. Finally, the evidence for "bioactivity" within ECM biomaterials is presented along with the aims of this study.

\subsection{The extracellular matrix}

Most living tissue exists in a gel-like state where different cells assemble within an ECM to execute their function. Figure 1-1 shows a falsely coloured scanning electron micrograph of a corneal stromal cell within a collagen ECM, published by Wilson et al. ${ }^{2}$. The ECM appears as a mesh of collagen fibres with many points of attachment to the cell. 

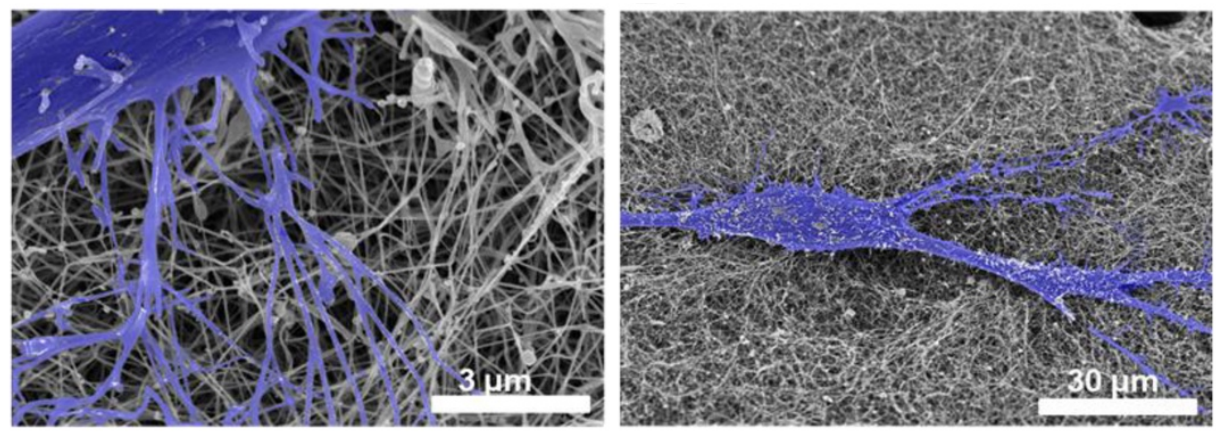

Figure 1-1 The extracellular matrix surrounding a cell.

\section{A corneal stromal cell (coloured) surrounded by an extracellular matrix which appears} as a mesh of collagen fibres reproduced from Wilson et al'.

Extracellular matrices can be interstitial connective tissue or basement membranes, defined by Keatch et al. as "interacting fibrous structures" compared with an "amorphous ground substance" respectively ${ }^{3}$. The former fills the space surrounding parenchymal cells, while the latter is a dense layer of ECM proteins separating the epithelium from the stroma. Perhaps the most important property of the matrix is its ability to withstand the many forces acting upon it including gravity, external mechanical forces and internal cell tension; whilst remaining elastic enough to provide tissue integrity and support for cell movement ${ }^{4}$.

\subsubsection{The composition of the extracellular matrix}

The ECM is composed of cell-secreted proteins assembled to form the foundation of each tissue. Hynes et al. compiled a "matrisome" of over 300 different proteins that make up the $\mathrm{ECM}^{5}$. ECM components include large structural proteins such as collagen, elastin and fibronectin and smaller functional moieties such as proteoglycans and growth factors.

\section{Collagens}

Collagen is the most abundant protein in the ECM and this protein gives the matrix its strength $^{6-8}$. There are 43 mammalian collagen subunits arranged into different tertiary structures in different tissues ${ }^{5,8}$. Collagens are expressed primarily by myofibroblasts as 
long repeating units of Gly-X-Y, where $\mathrm{X}$ is usually proline and $\mathrm{Y}$ is 4-hydroxyproline. The repeating pattern of the procollagen can be up to 1000 amino acids long, forming stable trimeric coils. Procollagens are clipped at the amino and carboxy terminal domains by procollagen peptidases. The long, chain-like collagen proteins associate with each other during fibril formation and are cross-linked by enzymes produced by fibroblasts (FB) and other cells. Multiple fibrils form fibres that are arranged into distinct structures in different tissue. Fibrillar collagens are found as tightly twisted right-handed helices in muscle, skin, bone and tendons. In basement membranes collagen fibres are arranged as laminar sheets. As shown in Figure 1-2, collagen fibres can form long, strong bundles giving the ECM the string-like appearance seen in Figure 1-1.

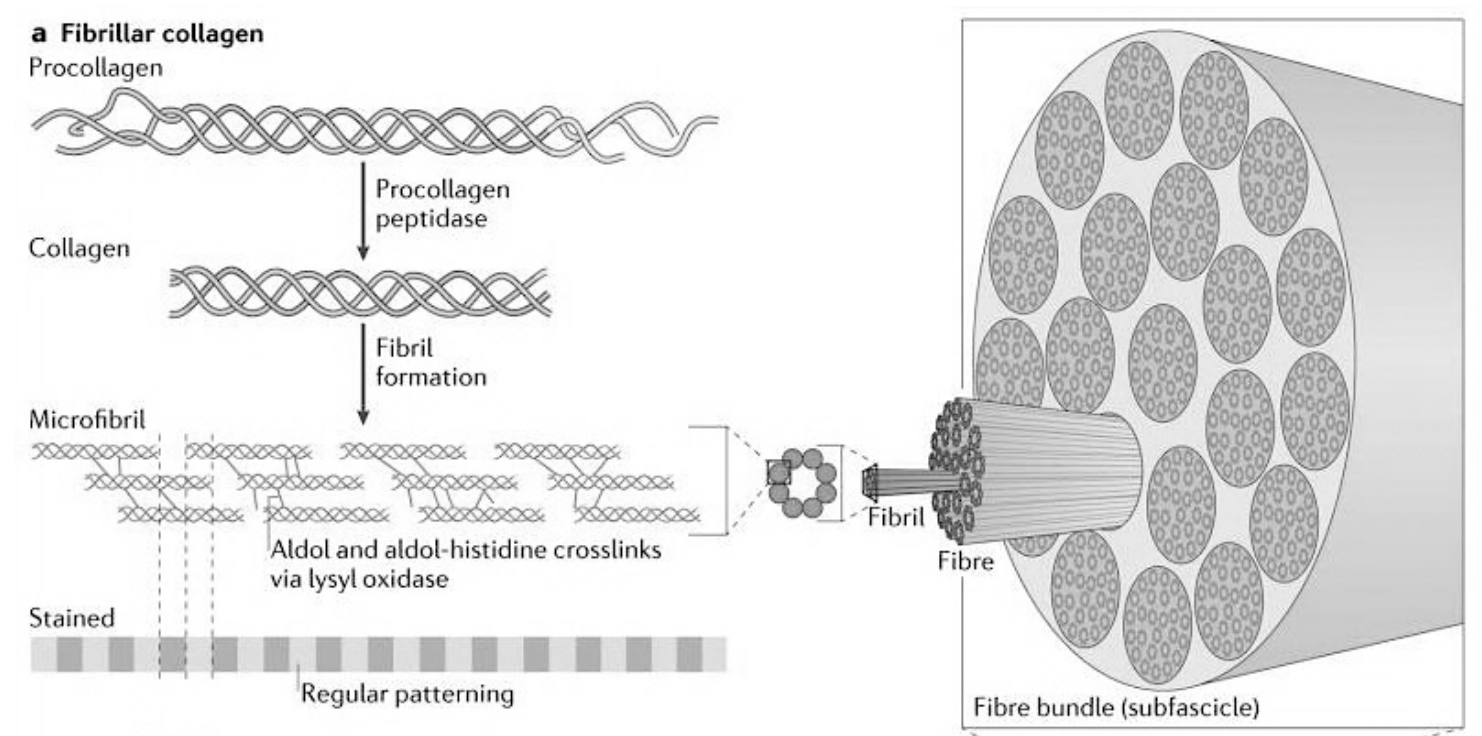

Figure 1-2 Fibrillar collagen

Myofibroblasts secrete long chains of procollagen which forms a triple helix. After amino and carboxyl domains are processed by endopeptidases, crosslinking enzymes form fibrils of multiple collagen helices. Fibrils assemble into strong fibres. Reproduced with permission from Mouw et al.

\section{Glycoproteins}

Over 200 distinct glycoproteins occur in the ECM that confer functionality to the matrix in different tissues ${ }^{5,8}$. Glycoproteins contain domains that interact with other ECM 
components during ECM assembly, they also possess domains that interact with cells. Major glycoproteins include elastin, laminin, fibronectin and tenascins. Elastin allows tissue to stretch and reform after movement, an important property in the ECM of veins and arteries ${ }^{9}$. Laminin and fibronectin are major components of the basal lamina. These glycoproteins make the ECM adhesive; binding to other ECM proteins such as collagen, and interacting with cells via integrins ${ }^{10,11}$.

Important vascular and blood glycoproteins include fibrinogen, vitronectin and vonWillebrand factor ${ }^{5,8}$. Fibrinogen is an important component of the granulation tissue during wound healing ${ }^{12,13}$. Vitronectin promotes cell adhesion and spreading, as well as controlling the plasmin system during clot formation ${ }^{14}$. VonWillebrand factor is an essential component of the blood clot which controls haemostasis and initiates the wound healing process ${ }^{15}$.

Nervous system glycoproteins include agrin, cochlin and netrins ${ }^{5,8}$. Agrins are involved in the development of neuromuscular junctions during embryogenesis. Cochlins form a major part of the inner ear matrix and netrins are involved in axon guidance.

\section{Proteoglycans}

Proteoglycans are glycoproteins with glycosaminoglycan (GAG) side chains ${ }^{8}$. Proteoglycans aggregate around collagen fibres where they sequester water and growth factors required by surrounding cells. GAGs are linear, charged polysaccharides consisting of repeating pairs of sugars, one of which is an amino sugar. The negatively charged GAGs act as space fillers within the ECM. Around 40 proteoglycans are found in the matrisome, which are divided into families based on their structure.

Leucine rich repeat (LRR) proteoglycans, such as decorin and biglycan, bind to collagens and growth factors ${ }^{16-19}$. As shown in Figure 1-3, LRR family proteoglycans contain GAG side chains and a repeating leucine rich sequence. The repeating sequence causes biglycan and decorin to have an arching structure. Decorin has only one GAG side chain of either dermatan or chondroitin sulphate, biglycan has two ${ }^{8}$. 

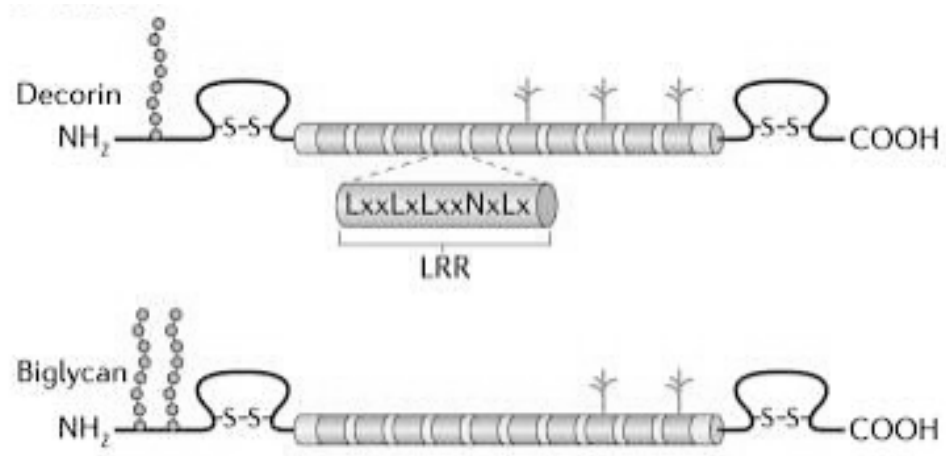

Figure 1-3 LRR family proteoglycans

Leucine rich repeat proteoglycans decorin and biglycan contain GAG side chains at the amino terminal end followed by a number of leucine rich repeats. Reproduced with permission from Mouw et al. .

\section{ECM modulators}

ECM-associated molecules such as enzymes and sequestered growth factors have important functions within the $\mathrm{ECM}^{5,8}$. Enzymes are fundamental for matrix assembly and disassembly. Hydroxylases and transglutamases are required for collagen cross-linking, while proteases such as procollagen peptidases, matrix metalloproteases (MMPs) and elastases are required for ECM break down. Growth factors bind to ECM proteins such as fibronectin and GAGs. They can act as chemokine gradients for important cells, or be presented to cells in order to initiate the activation of signalling molecules.

\subsubsection{Extracellular matrix function}

In some tissue, cells exist in a state of "anchorage dependence", in that they require the ECM for survival and proliferation ${ }^{3}$. This suggests that the function of the ECM is not limited to structural support and in many cases the ECM regulates cellular processes in a phenomenon known as "dynamic reciprocity" ",20,21. The phrase was first used by Bornstein et al. in 1982, the same year Bissel et al. proposed a model of ECM-cell interactions by transmembrane receptors ${ }^{21-23}$. The characterisation of integrins has demonstrated that a 
carefully regulated interplay occurs between cells, ECM and soluble factors, allowing tissue to withstand mechanical forces, maintain homeostasis and support cellular proliferation, migration and protein expression ${ }^{24-26}$. In describing the roles of the ECM it is useful to consider the interplay between the ECM, cells and soluble factors.

\section{Cell-ECM interactions}

The ECM can modify the phenotype of the cell via integrins ${ }^{23}$. Integrins are transmembrane receptors involved in both cell-to-cell and cell-to-ECM signalling ${ }^{24,26}$. Integrins communicate using a number of pathways to modulate migration, proliferation and gene expression. There are 24 known integrins, all of which are composed of $\alpha \beta$ heterodimers. Integrins bind to multiple ligands, primarily on collagen and laminin, linking the ECM to the cell cytoskeleton and intracellular signalling pathways (Figure 1-4).

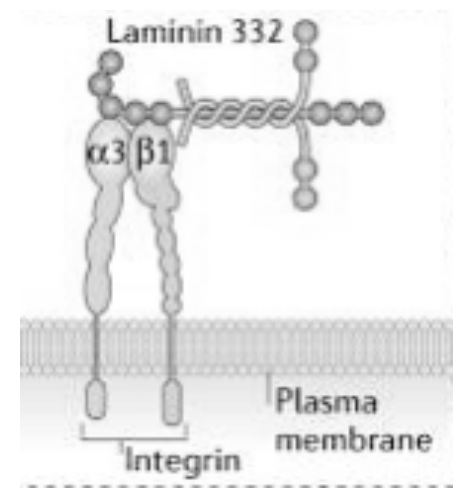

\section{Figure 1-4 Integrins connect the cell to ECM proteins}

Integrins are membrane bound dimers with binding sites for ECM proteins.

\section{Reproduced with permission from Mouw et al. ${ }^{8}$.}

The physical composition of the matrix can induce changes in surrounding cells. Holst et al. describe the "tensegrity architecture model", where calcium-mediated cell signalling is dependent on the tension interaction between integrins and cells ${ }^{27}$. Cells also sense disruption to the ECM; FBs are known to respond by expressing higher levels of migration stimulation factor in a "wound-like" matrix of denatured collagen and fibronectin, compared with intact collagen ${ }^{3}$. 
Conversely, cells can control the matrix around them. FBs synthesise collagen and other ECM proteins that then assemble into fibres ${ }^{28-30}$. The FBs remain intimately involved in controlling fibre contraction and matrix stiffness in a continuous feedback loop. Other cells are also involved in the degradation and re-assembly of the matrix; inflammatory cells, such as macrophages and mast cells release proteases to breakdown the matrix after injury. Infiltrating endothelial cells, keratinocytes and FBs release proteases to clear their path during tissue remodelling. Degraded matrix proteins will then guide the migration and proliferation of wound healing cells $\mathrm{s}^{31-33}$.

\section{Soluble factors-ECM interactions}

Cytokines, chemokines and growth factors are soluble regulatory molecules secreted by cells $^{34-36}$. The ECM is a vault of stored cytokines, including platelet derived growth factor (PDGF), transforming growth factor (TGF), endothelial growth factor (EGF) and fibroblast growth factor $(\mathrm{FGF})^{25,37,38}$. ECM proteins, especially GAGs and proteoglycans store growth factors and prevent them from binding to cell receptors until they are required. These factors are released when cells or proteases break down the ECM resulting in cellular activation or deactivation.

Some soluble factors are only effective if they are presented to a cell that is attached to a matrix. For example, FB chemotaxis towards an increasing concentration of a chemokine can depend on the substrate the FBs are growing on. A Transwell® chamber assay showed FBs growing on plastic migrated towards a gradient of PDGF, TG $\beta 1$, TGF $\beta 2$ and TGF $\beta 3$, while a sandwich assay indicated that FBs migrated towards only PDGF and TG $\beta 3$. The main difference in these methods is the presence of an ECM substrate in the sandwich assay. The investigators suggested that the attachment of either the cells or the growth factors to the ECM in the sandwich assay inhibited the chemotactic response to TGF $\beta 1$ and TGF $\beta 2^{3}$.

Proteases and protease inhibitors are associated with the ECM and control ECM synthesis, contraction and degradation. A great deal of research explores the interactions between 
proteases and the ECM during organ development, morphogenesis, normal tissue homeostasis, wound healing and cancer ${ }^{4,39-42}$. Some of the major proteases in wound healing will be discussed in relation to their roles in wound healing in section 1.3.

\subsection{An overview of wound healing}

The wound healing process can be divided into three overlapping stages: the inflammation stage (day 1-10), the formation of granulation tissue (day 3 to 15 ) and finally matrix remodelling (day $10-100$ ). A summary of these stages will follow, using information from a number of resources ${ }^{15,43-46}$.

\subsubsection{Stages in wound healing}

\section{Inflammation}

The immediate response to injury is to reform a protective barrier and prevent further blood loss ${ }^{15}$. Proteolytic cleavage of signalling molecules leads to the extravasation of blood vessels and the aggregation of platelets forming a fibrin clot. Platelet adsorption onto collagen is required for aggregation during "intrinsic coagulation" whilst "extrinsic coagulation" is activated by cell damage inducing the thrombin cascade. The fibrin clot recruits a number of cell types to the wounded area. Neutrophils and macrophages arrive first, their primary role being to phagocytose bacteria, debris and older neutrophils. They also play several important roles in the initiation of granulation tissue formation via the release of bioactive substances.

\section{Granulation Tissue}

Inflammation leads to granulation tissue formation as macrophages accumulate in the wound, FBs infiltrate from the wound's edge and endothelial cells start to form new vessels $^{47}$. The term "granulation tissue" refers to the granular appearance of the provisional matrix as it is populated by small blood vessels within the loose connective tissue. The 
presence of macrophages, FBs and new blood vessels are the result of signalling via both cellular and matrix related mechanisms, which lead to angiogenesis and fibroplasia ${ }^{48}$.

Re-epithelialisation at the wound's edge is activated by chemokines and growth factors released from wound activated cells, as well as the loss of cell-to-cell contact at the wound's edge and changes in structure of the ECM proteins ${ }^{49}$. The uncoiling of collagen fibrils reveals bioactive molecules within its structure and uncovers tri-peptide Arg-GlyAsp (RGD) binding sites that are essential for cell attachment during the re-colonisation of the granulation tissue by keratinocytes ${ }^{50,51}$.

Fibroplasia and wound contraction involves FB infiltration to the site of injury ${ }^{30,48}$. FBs deposit a fibronectin network that acts as an adhesive between other matrix components and incoming cells. Cells are able to move around and manipulate fibres in the loose fibronectin matrix more readily than in normal tissue. Growth factors stored in the ECM are released by cells and proteases, leading to FB recruitment, infiltration and fibronectin synthesis $^{12}$. Wound contraction is achieved by the conversion of FBs to myofibroblasts as they populate the granulation tissue and align with the collagen fibres at the edge of the wound. These actin rich cells use pseudopodia to attach to collagen and effectively pull the fibres at the edges of the wound closer together ${ }^{52}$.

Angiogenesis involves the sprouting of capillaries from surrounding blood vessels to provide the healing wound with essential nutrients and oxygen ${ }^{48,53}$. Endothelial cells at the tip of the sprouting edge of a new vessel are directed towards low oxygen, matrix fragments, growth factors and chemokines at the wound site, while cells at the border continue to proliferate. Both chemotaxis and proliferation of endothelial cells is essential in angiogenesis, but the direction of movement and the control of proliferation is tightly regulated. Angiogenesis during wound healing happens at the same time as fibroplasia and is dependent upon the process. Endothelial cells forming new blood vessels rely on directional cues provided by the granulation tissue matrix. Platelet and matrix derived factors such as heparin are involved in chemotaxis, proliferation and substrate support during the radial outgrowth of endothelial cells.

\section{Matrix remodelling}


Matrix remodelling is a constant cycle of collagen synthesis and assembly by FBs and myofibroblasts, versus collagen breakdown by enzymes, neutrophils, macrophages and other cells ${ }^{52,54}$. The transitional matrix of the granulation tissue is composed of fibronectin and hydrated hyaluronic acid. Durable collagen bundles replace the fibronectin, whilst non-sulphated hyaluronic acid is gradually replaced by sulphated GAGs containing a protein core that provides the new matrix with greater tensile strength. Myofibroblasts and FBs use the malleable transitional matrix to facilitate wound contraction by collagen fibrillation. Collagen units can be polymerised side-by-side and end-to-end during remodelling, resulting in long, strong fibres. Type I collagen generally makes up the fibril core, with types III and V collagen at the periphery of the bundles. Cells move around the collagen fibres forming larger stronger bundles, altering the intermolecular cross-links of the matrix and synthesising other ECM proteins.

Collagen begins to replace the granulation tissue around five days after injury ${ }^{15}$. Depending on the size and health of the wound, the resulting scar will only retain $70 \%$ the strength of the normal tissue and take three weeks to form. Collagen metabolism does not return to normal until 6 to 12 months after an injury ${ }^{55}$.

\subsection{Important contributors to the wound healing process}

Using histological and immunohistological methods, it is possible to identify important contributors to the wound healing process. Other methods, such as gene deletion mutants in mammal and zebrafish models, have demonstrated essential components in healthy wound healing ${ }^{56-60}$.

\subsubsection{Extracellular matrix components in wound healing}

In resting tissue, resident cells control the synthesis and deposition of ECM. The switch to a wounded state involves several changes relating to the overall matrix tension, exposure of bioactive ligands and the release of growth factors and cytokines ${ }^{61-65}$. Matrix and matrix associated proteins have several important roles. 
Fragments of ECM proteins, such as collagen and elastin, can promote migration, differentiation and proliferation of wound healing cells. The disruption of the basement membrane also breaks adhesion molecules between keratinocytes and proteins such as fibronectin, collagen IV and laminin, activating the keratinocytes ${ }^{51}$.

The breakdown of the provisional matrix proteins, especially GAGs and proteoglycans, can release signalling molecules which alter fibroplasia, angiogenesis and also the inflammatory response $e^{48,58,66,67}$. Heparin sulphate is broken down by heparinase to produce lower molecular weight fragments that promote the activity of $\mathrm{FGF}_{2}{ }^{68}$.

Hyaluronic acid promotes fibroplasia and angiogenesis in the granulation tissue in a number of ways $^{69-73}$. Hyaluronic acid promotes FB proliferation and stimulates the synthesis of matrix proteins. This protein also facilitates the motility of cells as they can easily attach to and detach from this component of the matrix as they move around ${ }^{74}$. Hyaluronic acid is also a useful space filler in the provisional matrix, as it can become hydrated. Finally, degradation products of hyaluronic acid have been shown to induce angiogenesis using a chick chorioallantoic membrane (CAM) model ${ }^{75-77}$.

\subsubsection{Proteases and protease inhibitors}

Many of the cells in wound healing express proteases that function to clear space, remove debris and remodel the $\mathrm{ECM}^{39,78,79}$. Epithelial and stromal cells express MMPs, serine proteases and cysteine proteinases (or cathepsins) to breakdown the ECM. The existence of 25 distinct MMPs is known in mammals. These include collagenases, gelatinases, stromelysins, matrisylins, metalloelastases, membrane type (MT-MMPs) and other MMPs $^{80-82}$. MMPs are expressed as pro-MMPs by keratinocytes, FBs, endothelial cells and inflammatory cells in response to cytokines, hormones and changes in contact with the ECM. Cleavage of the pro-MMP at the cysteine residue in the pro-domain consensus sequences PRCGVPD, leads to their activation. MMP activation is also regulated by TIMPS (tissue specific inhibitors of MMPs), other MMPs, serine proteases and plasma proteinase inhibitors. 
In addition to matrix proteins, MMPs interact with a number of other substrates including growth factors, cell adhesion molecules, receptors, cytokines other MMPs and other proteases $^{4,83}$. Wound healing proteinases can aid the wound healing process in the following ways:

- Degrade bulky ECM proteins

- Create space for infiltrating cells

- Cleave fragments to generate or reveal bioactive molecules

- Remove inter-ECM and inter-cellular junctions

- Modify cell-cell and cell-ECM signals

MMP-1 is expressed at the wound healing edge by FBs and activated keratinocytes ${ }^{84}$. MMP- 1 expression is instigated by the interaction between the ECM and the $\alpha 2 \beta 1$ integrin receptors of the keratinocytes after injury. Neutrophils express MMP-9, which is one of the most abundant MMPs in the cutaneous wound ${ }^{85}$. MMP-12 is expressed by wound healing macrophages and detected around blood vessels in the healing wound.

The expression of MMPs is tightly controlled in the healing wound as MMP-1 overexpression is a hallmark of chronic inflammation. An imbalance in the activity of MMPs and TIMPs results in a destructive, chronic wound environment. Factors such as age, diabetes and the presence of persistent leukocytes may contribute to the uncontrolled expression of MMP-1 in chronic wounds ${ }^{78,86}$.

Adamalysins, also known as "shreddases", include ADAMs (a disintegrin and metalloprotease) and ADAMTs (a disintegrin and metalloprotease with thrombospondin motifs). These enzymes have important roles in ECM control including the release of cell receptors, adhesion molecules and growth factors via cleavage of transmembrane protein ectodomains $^{4,87}$. Meprins regulate ECM remodelling by activating other proteases as well as cleaving basement membrane proteins such as collagen IV and fibronectin. 


\subsubsection{Matrikines and Matricryptins}

Another role of MMPs is the release of bioactive fragments of ECM proteins involved in tissue remodelling and angiogenesis ${ }^{88-92}$. These are known as matrikines and matricryptins; ligands derived from subdomains of matrix proteins, capable of modulating cell signalling. The difference between matrikines and matricryptins is in their bioavailability. Matrikines are specific regions of ECM proteins that alters cell behaviour after ligand-receptor binding. Matricryptins must be released from their parent proteins by proteolytic cleavage, cellular processing or after a conformational change in the matrix proteins, thus releasing or exposing the ligand that was previously unavailable to cells.

These ligands are distinct from soluble growth factors and cytokines ${ }^{90-92}$. They often act with a lower binding affinity, in the $\mathrm{mM}$ range, compared with cytokines (which usually act in the $\mathrm{nM}$ range). This is compensated for by the fact that the ligand can occur as a tandem repeating sequence and because it is not internalised or depleted by the target cell. In addition, cells attached to the ECM are often in close proximity to the matrikine or matricryptin, unlike growth factors which form gradients from a distance.

The discoidin domain receptor (DDR) binding sequence of collagen is a well characterised matrikine ${ }^{88,91,93}$. The binding of this ligand to the DDR-1 in epithelial cells leads to smooth muscle cell migration. Binding of the same consensus to DDR-2 in mesenchymal stem cells induces the production of MMP-1, leading to the degradation of nearby fibrillar collagen. Another example of a matrikine is the leucine rich repeat (LLR) domain in decorin, which binds to the EGF receptor (EGFR) supressing EGFR expression and EGFR auto phosphorylation. This is thought to act as a "quiescent signal" decreasing migration and proliferation in the mature wound.

Matricryptins, require modifications to their ECM parent protein before their ligand is available to cell receptors ${ }^{90-93}$. EGF-like repeats are present on laminin 5, but this ligand must be made available by the action of MT1-MMP and MMP-2 before it will apply its biological function. Other examples of matricryptins include endostatin, released from Collagen XVIII by elastase, cathepsins and MMPs; tumstatin, released from Collagen IV 
by MMP-9; and the XGXXPG consensus of elastin, released by MMP-2, MMP-9, MMP-7 and MMP-12.

1.3.4 Cells, cytokines, chemokines and growth factors in wound healing

The change from resting to injured tissue involves the activation of cells and the ECM to respond to the change in the environment. This is often achieved by the release of signalling molecules. Numerous cytokines and growth factors are involved in important wound healing processes including angiogenesis, chemotaxis, proliferation, collagen synthesis and inflammation ${ }^{34-36}$. These soluble molecules are expressed by cells in the wound and surrounding tissue and can also be stored by the ECM.

\begin{tabular}{|c|c|c|c|}
\hline MEDIATOR & SOURCE & CELL RESPONSE & IMPACT \\
\hline EGF & $\mathrm{K}, \mathrm{M}, \mathrm{P}$ & $\begin{array}{l}\text { PROLIFERATION (FB, EC) } \\
\text { CHEMOTAXIS (EC) }\end{array}$ & $\begin{array}{l}\text { ANGIOGENESIS, RE- } \\
\text { EPITHELIALISATION, } \\
\text { COLLAGEN SYNTHESIS }\end{array}$ \\
\hline PDGF & $\mathrm{P}$ & $\begin{array}{l}\text { PROLIFERATION (FB) } \\
\text { CHEMOTAXIS (MONO, FB) }\end{array}$ & $\begin{array}{l}\text { INFLAMMATION, RE- } \\
\text { EPITHELIALISATION, } \\
\text { COLLAGEN DEPOSITION } \\
\text { TISSUE REMODELLING }\end{array}$ \\
\hline FGF & $\mathrm{K}, \mathrm{FB}, \mathrm{EC}$ & $\begin{array}{l}\text { PROLIFERATION (MONO, } \\
\text { FB, EC) } \\
\text { CHEMOTAXIS (FB, FB) }\end{array}$ & $\begin{array}{l}\text { ANGIOGENESIS, } \\
\text { INFLAMMATION, } \\
\text { COLLAGEN SYNTHESIS }\end{array}$ \\
\hline TGFB & $\mathrm{FB}, \mathrm{K}, \mathrm{M}, \mathrm{P}$ & $\begin{array}{l}\text { PROLIFERATION (EC, FB) } \\
\text { CHEMOTAXIS (MONO, FB) }\end{array}$ & $\begin{array}{l}\text { INFLAMMATION, } \\
\text { ANGIOGENESIS, } \\
\text { COLLAGEN SYNTHESIS }\end{array}$ \\
\hline VEGF & $\mathrm{K}, \mathrm{FB}, \mathrm{M}, \mathrm{EC}$ & $\begin{array}{l}\text { PROLIFERATION (EC) } \\
\text { CHEMOTAXIS (EC) }\end{array}$ & $\begin{array}{l}\text { INFLAMMATION, } \\
\text { ANGIOGENESIS }\end{array}$ \\
\hline IL-1 & $\mathrm{M}, \mathrm{L}, \mathrm{K}, \mathrm{FB}$ & $\begin{array}{l}\text { PROLIFERATION (FB) } \\
\text { CHEMOTAXIS (MONO) }\end{array}$ & $\begin{array}{l}\text { INFLAMMATION, } \\
\text { ANGIOGNENESIS, } \\
\text { COLLAGEN SYNTHESIS, } \\
\text { TISSUE REMODELLING }\end{array}$ \\
\hline
\end{tabular}

Table 1-1 Soluble mediators of wound healing

Key: FB- fibroblast, EC- endothelial cell, K-keratinocyte, Mono-monocyte, Mmacrophage, L-leukocytes, P-platelet 
Table 1-1 summarises a few of the most important mediators of wound healing, using information from Barrientos et al. and Behm et al. ${ }^{34,35}$. The first column gives the name of the growth factor, cytokine or chemokine, the following columns show the cells which produce the mediator, the target cell or cells and the biological consequence with regard to wound healing.

\section{Platelets and endothelial cells}

Platelets begin the wound healing process when they form a clot to reduce blood loss. This clot contains platelets, chemokines and growth factors. The normal platelets are activated by this aggregation to form spidery granulated cells that release "wound" signals in the form of growth factors and cytokines including PDGF and TGF $\beta^{15,58,94}$.

Endothelial and endothelial progenitor cells are activated by Interleukin 1 (IL-1) and interferon gamma (IFN $\gamma$ ) in the capillaries surrounding the wound area. This decreases blood flow and activates key adhesive molecules, P-selectin and E-selectin, along with the intercellular adhesion molecule (ICAM), to trap circulating neutrophils that migrate through the capillary wall to the wound area ${ }^{53,94}$.

\section{Leukocytes}

Neutrophils arrive to the site early, peaking in number between 24 and 48 hours after injury $^{15,58,95}$. These cells release free radicals, reactive oxygen species, cationic peptides, eicosanoids, and histone deoxyribonucleic acid (DNA) nets to combat pathogens. They also begin to clear the area of microbes and debris by phagocytosis ${ }^{94}$. Although this part of the process is very important for the immediate response to infection, if uncontrolled it can be detrimental to the surrounding tissue, as seen in chronic wounds. One notable aspect of foetal healing is the decrease in neutrophil recruitment by endothelial cells to the wound healing site ${ }^{96}$. In the 1960 s a model of wound healing using neutropenic mice showed there was no delay in wound closure, indicating that these cells may not be essential to the wound healing process ${ }^{97}$. Similarly, mast cells, which are derived from circulating 
basophils, have an important role in the generation of pro-inflammatory mediators and cytokines that induce changes in vascularity. However, their effect on wound healing, in the absence of infection is debatable $\mathrm{e}^{32,98}$.

Macrophages arrive later than neutrophils from circulating monocytes and surrounding tissue $^{99}$. Fragments of collagen, elastin and fibronectin, as well as enzymatically active thrombin recruit macrophages. Macrophages are activated by growth factors released from the blood clot and fragments of ECM proteins. Neutrophils, platelets and FBs at the wound site, which have begun to breakdown ECM proteins, generate a chemotactic gradient of bioactive molecules that attract resident tissue macrophages. Macrophages arriving at the wound site are termed "mature" tissue macrophages, having an altered phenotype. Mature macrophages begin phagocytosis and antigen presentation, but also amplify the wound healing signals by producing cytokines, growth factors and angiogenic factors. Many of the growth factors and cytokines released by the macrophages induce FBs to proliferate, migrate and synthesise ECM proteins. Macrophages and monocytes also interact with progenitor cells during the formation of fibrovascular bundles ${ }^{100}$. Another important role of the macrophage is to clean up the site of cell debris such as apoptotic neutrophils and matrix fragments. The role of macrophages will be discussed in more detail in section 1.5.

\section{Keratinocytes}

The relatively static function of the normal keratinocyte is to maintain tissue integrity while providing a protective barrier from physical trauma, temperature and chemical damage $^{15,51}$. Sheets of keratinocytes are disrupted in a cutaneous injury causing detachment from the ECM proteins that ordinarily hold them in place. This induces activation of the keratinocytes into a proliferative and migratory phenotype ${ }^{51}$. Changes in integrin expression allows keratinocytes to extend lamellipodia across the provisional matrix, as they are no longer tethered to a basement membrane. Leading edge cells migrate over the provisional matrix and those behind proliferate, such that the cells are dragged as a sheet to close the gap, manufacturing new basement membrane proteins as they go. Leading edge keratinocytes release plasmin to clear the fibrin clot ahead of them ${ }^{101}$. 
Fibroblasts, myofibroblasts and pericytes

FBs exist in a quiescent form in resting tissue ${ }^{30,54}$. Their main role is to synthesise ECM proteins, remodel the ECM with proteases and control the tension of collagen fibres. Resting FBs are activated by the interaction between FBs and keratinocytes, the change in mechanical tension of the environment and the presence of platelet derived growth factors. During wound healing, FBs from surrounding tissue, resident precursors such as pericytes and bone marrow derived MSCs differentiate into myofibroblasts. These cells are responsible for the construction of granulation tissue, which they achieve by expressing ECM proteins, protease inhibitors and fibrogenic mediators such as FGF $^{102}$. Myofibroblasts contract the wound during scar formation by forming strong bonds to the new collagen fibrils. They use actin rich networks to form focal adhesions, and an integrin based system to connect cells with ECM. The late stages of wound repair are marked by changes in protease expression, growth factor induction, mechanical tension and cell density, causing a decrease in myofibroblast numbers as they are depleted via apoptosis. This process has been modelled in vitro using free-floating collagen gel lattice FB cultures $^{28,29,103,104}$. The FB phenotype can be pro-migratory or pro-contractile; a rapid shrinkage of the gel is observed relative to the the gel stiffness and cell density. Growth factors can also control the FB phenotype; PDGF inducing a migratory response while lipoprotein A (LPA) and foetal bovine serum (FBS) induce contraction of the gel by $\mathrm{FBs}^{105}$. Vascular pericytes are derived from committed precursors or recruited by endothelial cells to the wound site. They are activated to proliferate and migrate towards the leading edge endothelial cells and the PDGF gradient from the wound. They wrap around endothelial cells during angiogenesis, giving strength and movement to the new vessel ${ }^{106}$. Pericytes are thought to be a possible source of mesenchymal stem cells in tissue ${ }^{107,108}$.

\subsection{Mesenchymal stem cells}

The concept of stem cells in mammalian tissue is concerned with "plasticity" of different cell types or their ability to renew and differentiate. This was first observed in the 
hematopoietic stem cell (HSC), which is a common progenitor for blood cells ${ }^{109,110}$. Research from the late 1950s and early 1960s demonstrated the first sign of a nonhematopoietic stem cell ${ }^{110}$. After world war II, scientists became interested in treatments for radiation involving the transplantation of bone marrow from untreated mice to radiated mice $^{111}$. The first human bone marrow transplant was performed in 1957 by Thomas et al. ${ }^{112}$. In 1961, Freidenstein isolated rodent bone marrow cells that were fibroblast-like in appearance, adherent to plastic, formed colonies in methyl cellulose medium, and were capable of osteogenic differentiation ${ }^{113,114}$. In the following years, it was shown that these bone marrow stromal cells were capable of differentiating into multiple lineages and thus the mesenchymal stem cell was identified ${ }^{115}$. Mesenchymal stem cells are so named because they differentiate into mesoderm lineages. The mesenchymal stem cell (MSC) was defined in 1991 as a "putative stem cell for skeletal tissue found in bone marrow stroma" or, "stem cells for non-hematopoietic tissue"

It soon became clear that multi-potent cells could be found in other tissue such as fat, skin, synovial membrane, dental pulp and the umbilical $\operatorname{cord}^{107,115,116}$. The significant variations in gene, cytokine and receptor expression observed between populations of MSCs led to the establishment of a minimum criteria for MSC identification in $2006^{117}$ :

- Adherence to plastic

- Express CD105, CD73, CD90

- Do not express CD45, CD4, CD14, CD11b, CD79, CD19 HLA-DR

- Capable of differentiating into osteoblasts, adipocytes and chondroncytes in vitro.

\subsubsection{MSCs in wound healing}

In the adult bone marrow, MSCs are a pool of regenerative cells that are capable of selfrenewal and play an important role in tissue repair ${ }^{118-120}$. Firstly, they are capable of differentiating into cells that are required at the site of injury. They support haematopoiesis by maintaining the HSC niche. They release cytokines that modulate the inflammatory 
response and trophic factors that promote healing processes such as cell recruitment, angiogenesis and collagen synthesis.

MSCs, along with FBs, fibrocytes and pericytes can differentiate into myofibroblast progenitors that are stimulated by cell matrix interactions, matrix stiffness and mechanical stress to become myofibroblasts - the primary matrix producing cells in wound healing ${ }^{102}$. A study using labelled donor bone marrow derived stem cells showed that after wound healing, labelled cells had formed pericytes, endothelial cells and keratinocytes ${ }^{119}$.

As well as being able to differentiate into the type of cells required at the site of injury, MSCs have important roles in the regulation of other wound healing cells ${ }^{120}$. MSCs increase the rate of FB migration, proliferation and collagen synthesis as well as endothelial cell tube formation. During the inflammatory stage of wound healing, MSCs regulate the immune response by blocking T-cell proliferation, producing IL-10 and IL-4. During the proliferation stage they release paracrine signals to recruit keratinocytes, dermal FBs and other nearby stem cells. They secrete important wound healing growth factors such as keratinocyte growth factor (KGF), VEGF and PDGF. Finally, during remodelling, MSCs regulate the expression of MMPs and collagen deposition.

\subsubsection{MSC Migration}

MSCs migrate from the bone marrow and other healthy tissue to the site of injury via chemotactic signalling. MSCs exit the bone marrow into the blood stream via the SDF1/CXCR4 system ${ }^{121,122}$. SDF1 (stromal cell derived factor 1) is a chemokine that is expressed in bone marrow and injured tissue. The MSC receptor $\mathrm{CXCR} 4$ (C-X-C motif) binds to SDF1 and recognises the tissue injury homing signal, allowing detachment from other MSCs and the bone marrow stroma by MT 1-MMP expression, releasing the MSCs into the blood stream. Signals from the injured tissue recruit the stem cells to the injury site. Ratajctak et al. published a review describing several "priming" factors that appear to increase the sensitivity of the SDF-1 gradient ${ }^{123}$. These factors are derived from wound 
"signals" such as fragments of complement cascade peptides, antimicrobial peptides secreted by myeloid cells, hyaluronic acid and fibronectin.

MSCs find the injured tissue by passive homing, active homing or active arrest ${ }^{122,124}$. Passive homing occurs when MSCs naturally decelerate in capillaries due to their size in relation to the narrowing capillary. During active deceleration, MSCs recognise inflammatory signals from surrounding tissue and migrate by tethering to these on the vessel wall or rolling by the attachment of VCAM (vascular cell adhesion molecule) and Pselectin. Active arrest occurs when the MSC flattens and spreads on the endothelium at the site of injury. Whichever means the MSC uses to recognise the injury site, the cells then transmigrate through the endothelium wall into the tissue, a process mediated by MMP activity.

\subsection{Wound healing, scar formation and tissue regeneration}

The process described is an overview of the stages of normal wound healing in humans and animals. In reality, the process can be more complex. To start with, different organs in the body are divergent in terms of their ability to heal after injury. The regenerative capacity of an organ is related to a number of factors including the proximity of stem cells $^{116,125}$. Blood is replenished at regular intervals and blood loss, to a certain extent, is tolerated as the body is able to replenish its supplies in a matter of weeks. Liver tissue will regenerate after the removal of up to $50 \%$ of its mass ${ }^{126,127}$. Damage to the myocardium leads to fibrotic tissue that is not as strong as healthy tissue and this can lead to further complications with age, such as blockages ${ }^{128}$. Neural tissue is practically non-regenerative, extensive damage to the spinal cord for instance cannot be repaired and tissue function is rarely restored after damage. The ability to repair muscle, joint, skin and bone tissue in mammals depends on a number of factors, including the extent of damage as well as the age and health of the animal $1^{125,127}$.

In cutaneous tissue, wound healing occurs as described in the absence of infection and other interfering pathologies such as diabetes ${ }^{45,129,130}$. Small burns, cuts and abrasions heal completely leaving a normal regenerated dermis and epidermis, although without 
appendages such as hair follicles. Larger wounds, second and third degree burns for instance, can results in scar formation by fibrosis. Fibrosis can occur in most tissue as the result of prolonged chronic inflammation, causing excess deposition of ECM components by the myofibroblasts ${ }^{131}$. The fibrotic scar tissue is not as strong or elastic as the original tissue $^{127}$.

A breach in cutaneous tissue leads to the exposure of environmental pathogens, leading to a risk of infection if the wound is not sealed quickly ${ }^{12,63}$. Extensive burns wounds, especially, can be complicated by infection preventing the normal constructive remodelling to occur. Uncontrolled infection can lead to sepsis and death, but when the infection is contained to the wound site the host response to infection becomes the priority over constructive wound healing.

Another barrier for cutaneous wound healing is the existence of other pathologies. Diabetic ulcers are particularly difficult to treat when they fall into a "chronic" wound status as the body is unable to heal the wound on its own. The non-healing wound becomes overrun with immune cells secreting uncontrolled levels of proteases leading to a disruption of normal collagen synthesis and matrix remodelling ${ }^{129,130,132}$.

Another complication to normal tissue regeneration is the age of the individual. For a number of reasons, including the build-up of fibrotic tissue from previous damage, the regenerative capacity of most tissue will decline with age ${ }^{131}$. Recently, studies in wound healing have shown that foetal wounds do not lead to scar formation and heal completely. This may be due to differences in the balance between constructive regeneration and the immune response, amongst other factors ${ }^{95,96,98}$.

\subsubsection{The importance of the immune response during healing}

In mammals the skin is a suitable barrier to infection, but cutaneous injury results in exposure to a plethora of microbes ${ }^{94,133}$. The rapid response of the body described in section 1.2 is a constructive mechanism that first prevents blood loss and then rebuilds the damaged tissue. In parallel, the body mounts an immune response to the presence of 
microbes and other foreign particles or debris. Wound closure requires a balance between these processes.

\subsubsection{Innate immune response to infection}

Leukocytes in the blood stream are activated by injury to provide an immune response. Vaday et al. describe them as being in a state of "constant surveillance" ready to facilitate a response $^{38}$. When tissue is compromised, changes in the ECM promote the extravasation of leukocytes from the bloodstream to the affected area.

Activated leukocytes at the site of injury are able to mount an inflammatory response to capture, neutralise and remove foreign microbes $^{44,45}$. Neutrophils, mast cells and macrophages are activated and use different means of first destroying the foreign antigens, and then starting a signal cascade to recruit other immune cells. An infection that can be dealt with swiftly by leukocytes leaves FB and endothelial cells free to begin building the granulation tissue and initiate matrix remodelling. A wound free of neutrophils five days after injury indicates that any infection has been cleared and inflammation controlled ${ }^{133}$. When the wound is occupied by foreign material, debris, bacteria and dead cells, the immune response continues to amplify. This can set back the rebuilding process for an extended period of time and lead to even greater destruction of the tissue.

In response to injury, the ECM regulates the transition of integrins to a state of high binding affinity, allowing greater leukocyte adhesion in the area. The interaction between integrins and leukocytes promotes the release of pro-inflammatory cytokines and $\operatorname{proteases}^{47,134}$.

An important role of leukocytes in wound healing is the degradation of the ECM. As previously discussed, a number of matricryptins with alternate activity from their parent protein exist in the ECM. The digestion of the ECM by leukocyte proteases can expose active sites that are required for tissue remodelling. Peptides from laminin, elastin, collagen and hyaluronic acid can lead to monocyte and neutrophil recruitment, cytokine production and protease production ${ }^{92}$. A number of other inflammatory mediators are 
bound to ECM components and released by injury or proteolysis. Decorin bound TFG $\beta$ acts on $\mathrm{T}$ cells, $\mathrm{B}$ cells, endothelial cells, macrophages and $\mathrm{FBs}^{135,136}$. Heparin sulphate binds growth factors and chemokines including CCL5, which acts on memory T cells, eosinophils, basophils, natural killer cells and dendritic cells ${ }^{38,137}$.

\subsubsection{Macrophages in tissue reconstruction}

The role of leukocytes in wound healing as pro-inflammatory mediators is well established $^{133}$. It was previously understood that the primary role of the macrophage in wound healing is to neutralise pathogens and clear debris, such as dead neutrophils, erythrocytes and fibrin. Studies in the 1970s demonstrated that macrophage depleted mice displayed delayed wound closure ${ }^{138,139}$. The key finding in these studies demonstrated that macrophages also had an impact on FB remodelling of the wound matrix, linking the distinct processes of inflammation and constructive remodelling. Macrophage depleted wounds led to a delay in FB recruitment and a reduction in FB proliferation. Later it was shown that macrophages were key producers of PDGF and other "fibrogenic cytokines" and they modulated FB collagen synthesis ${ }^{99,138}$. Macrophages were also shown to control recruitment and proliferation of other wound healing cells, such as vascular cells ${ }^{100}$.

All of these studies described the wound healing macrophages as "activated macrophages" compared with circulating monocytes and resting tissue macrophages because it was clear that their response to injury involved a change in phenotype. Later studies showed that macrophages could be activated in different ways, known as classical (M1) or alternative (M2) activation 99,140 . Classically activated macrophages (M1) are induced by LPS, IFNץ, TH1 cells and other M1 macrophages. They release superoxide, hydrogen peroxide, Il-12 and Il-33 to kill bacteria; as well as cytokines to amplify the inflammatory response. The M2 macrophages are activated by Il-4 and IL-13 expressed by mast cells and TH2 cells. They contribute to constructive remodelling by recruiting FBs, endothelial cells and MSCs and promoting collagen synthesis as summarised in Figure 1-5 ${ }^{141}$. 


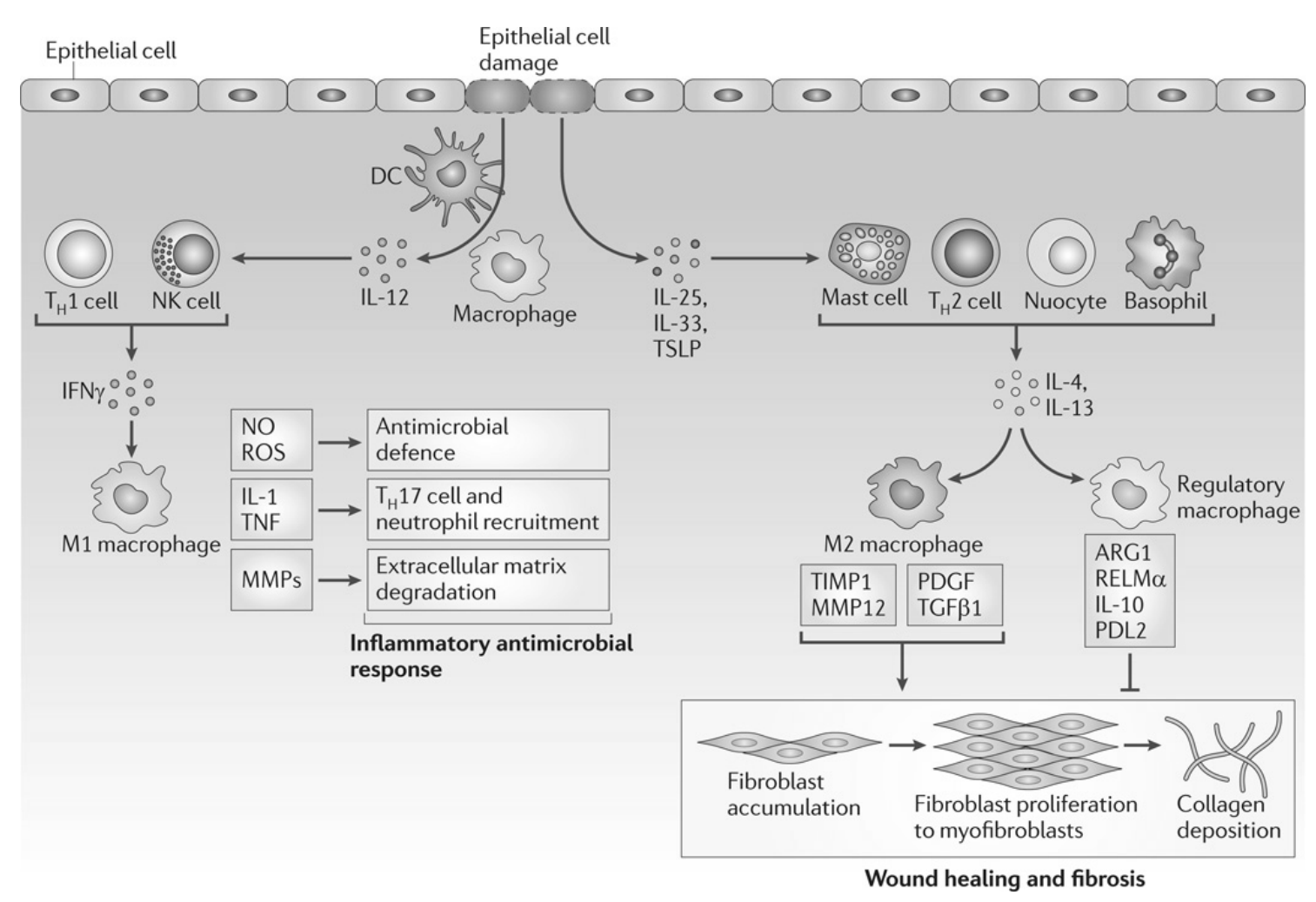

Figure 1-5 Macrophage phenotypes in wound repair and inflammation

Tissue damage triggers activation of macrophages into an M1 or M2 phenotype. Dendritic cells (DC), Helper T cells (Th1) and natural killer cells (NK) release IFNץ which promotes an inflammatory response in the macrophage. Mast cells, Th2 and basophils release IL-4 and IL-13 promoting an M2 phenotype. M2 macrophages express growth factors to recruit FBs and promote collagen synthesis. Image used with permission from Murray et al. ${ }^{141}$.

The dichotomy of M1 and M2 macrophage activation has given way to a more complex model that accounts for the heterogeneity seen within the macrophage population ${ }^{140,142-145}$. Alternatively activated macrophages now fall into one of three classes (M2a, M2b or M2c) depending on how they are activated. IL-4 and IL-13 induce the M2a phenotype, immune complexes and TLR ligands lead to $\mathrm{TH} 2$ activation promoting an M2b phenotype, and finally, IL-10 leads to the M2c phenotype. These three subsets promote tissue remodelling by releasing the deactivating cytokines IL-1o and TFG $\beta$, and by producing lower levels of inflammatory cytokines. The relative levels of M1 to M2 macrophages in the population dictate the response within the wound as either pro-inflammatory or anti-inflammatory; 
being destructive, or constructive, respectively. The activated macrophage profile has become a useful way to determine the health of a wound. During chronic inflammation, the majority of macrophages are of the M1 type, while in a healthy healing wound, M2 macrophages prevail ${ }^{130,132,141}$. This is consistent with studies showing that the majority of the macrophages in the foetal healing wound are M2 activated and express the constructive phenotype $\mathrm{p}^{146,147}$

\subsubsection{The foreign body response}

As well as fighting infection, immune cells respond to materials that they recognise as "non-self" 148,149 . This can be debris caught up in the wound or foreign objects such as bullets and blades. In addition, medical devices used to replace diseased or damaged tissue will illicit a foreign body response.

When a foreign object is introduced into tissue, the entire surface is immediate adsorbed by proteins, such as fibrin, fibronectin, vitronectin, opsonins, albumin and complement activated fragment $\mathrm{C} 3 \mathrm{~b}^{148,149}$. Inflammatory cells from the surrounding tissue, including monocytes, platelets and neutrophils adhere to the proteins and release pro-inflammatory signals to facilitate the removal of foreign bodies from the tissue.

Neutrophils, which would normally phagocytose microbes and then be cleared away within two days, now remain in the vicinity longer; producing proteases and oxygen free radicals. Macrophage cells, responding to a foreign body much larger than the microbes they typically engulf, fuse with each other to form giant cells as they attempt to neutralise the foreign body. The appearance of such giant cells in tissue and the extended presence of neutrophils are signs of chronic inflammation. FBs that are unable to penetrate the foreign body, will eventually form a 50-200 $\mu \mathrm{m}$ thick fibrotic layer around the material, similar to scar tissue. When biomaterials are introduced to the body in any capacity the intensity of the foreign body response will dictate the successful remodelling of the injured tissue ${ }^{148}$. 


\subsection{Biomaterials in medicine}

\subsubsection{Historical uses of biomaterials in humans}

A biomaterial is defined as "a non-viable material used in a medical device, intended to interact with biological systems" ${ }^{150}$. Although this term was established in the 1960s, there are many examples of biomaterials that are thousands of years old. The subject is explored thoroughly by Ratner et al., who traced some of the oldest examples of biomaterials known to mankind ${ }^{150}$. Dental implants made from seashells, gold, ivory and other metals have been dated as far back as the Mayan era. Sutures made from catgut, were used in Europe during the middle ages and biting ants were used to help close wounds in India and South Africa.

In the 1880 s, experiments with metals and alloys showed that some materials were "tolerated" better by the human body than others and the concept of biocompatibility was established ${ }^{148-150}$. After World War II, the use of different plastics in devices emerged as the materials became available. Harold Ridley noted that pilots who sustained eye injuries from shattered windscreens demonstrated a "tolerance" to the material embedded in their eyes. This led Ridley to use this plastic (Perspex) as an artificial lens in patients after cataract surgery, a procedure that is still used today ${ }^{151}$. Other surgeons at the time were using different materials to replace diseased or damage tissue; such as ceramics as hip implants and metals and alloys for replacement heart valves.

As scientist and doctors have begun to understand the underlying processes involved in wound healing, tissue regeneration and the "foreign body response", the field of biomaterials has grown to include devices designed to replace or repair almost every tissue type in humans $3,148,150$. 


\subsubsection{The scope of modern day biomaterials}

The ever-growing field of biomaterials now encompasses a vast array of devices for tissue replacement and rebuilding ${ }^{150}$. While there is no ideal biomaterial, different classes of biomaterials have qualities that render them suitable for particular uses. Ultimately, success or failure of a biomaterial is determined by two factors ${ }^{148,152}$;

- Biocompatibility: it must not illicit a detrimental foreign body response.

- Usability: it must have the appropriate physical attributes to replace the diseased tissue, be it strength, elasticity or transparency.

Tolerance or biocompatibility is essential as a foreign body will trigger a host immune response that can retard normal healing ${ }^{148}$. Although the mechanisms were not understood initially, it was clear to pioneers in this field that some materials were tolerated by the body better than others. Surgeons of the time attributed the differences to the presence of leachable impurities.

Some inert metals, such as gold, were not readily available or did not possess the desired physical attributes to make an appropriate biomaterial. Some natural materials, such as catgut, were tolerated well compared to others. However, effective methods of sterilisation were not implemented until the last century with the result that relatively well-tolerated materials contaminated with bacterial and viral particles could trigger an immune response $^{148,150}$.

The physical attributes of the material are important for tissue repair or replacement ${ }^{149,153}$. Replacing hard tissue such as bone, teeth and joints requires a strong, durable scaffold made of metal, plastic, ceramic or minerals such as hydroxyapatite. Hydrogels, polymers and sponges are more suitable for use in soft tissue repair such as skin and ocular lenses.

There are now many different biomaterials used in medicine, the most common are catheters, hip and knee prostheses, breast implants, intraocular lenses, contact lenses, heart valves and stents, pacemakers, renal dialysers and synthetic skin materials. 


\subsubsection{Natural and synthetic biomaterials}

Biomaterials are composed of natural or synthetic materials. Natural biomaterials include proteins (such as collagen, fibrin or silk) and polysaccharides (such as hyaluronic acid, chitosan, alginate and agarose). Synthetic materials can be organic polymers such as polyethylene glycol, or inorganic materials such as ceramics and metals. Some biomaterials are composites, for example collagen coated metal stents. The metal is strong enough to prevent vascular collapse, while the collagen reduces the foreign body response $\mathrm{e}^{150,153}$.

Synthetic materials can be easily honed and manipulated for a desired purpose. Using modern three dimensional printing methods, materials can be generated to topographically imitate the surface properties of normal tissue ${ }^{154}$. In addition, strategies have been employed to render synthetic materials more tolerant in the body. These include surface modifications such as non-fouling surfaces that deter protein adsorption and specific RGD binding sites to promote cell attachment ${ }^{155}$.

Natural biomaterials can be made from many different proteins, including proteins that are derived from ECM tissue such as collagen and fibrin ${ }^{7,156}$. Natural biomaterials range from polymers to complex matrices reminiscent of normal $\mathrm{ECM}^{3}$. One of the most attractive features of natural materials is that they will be incorporated into normal tissue without eliciting a detrimental foreign body response ${ }^{148}$. In addition, there is growing evidence that some materials play a positive role in the normal healing process, above that of a support for cellular infiltration. Given the many ways in which ECM proteins are involved in wound healing, it stands to reason that a biomaterial composed of ECM proteins will contribute to the constructive remodelling process ${ }^{3,152,157}$.

\subsection{Focus on ECM based biomaterials}

One way to consider biomaterial design for tissue regeneration is to establish a top down or bottom up approach. A bottom up approach uses the surface science, mechanics and chemistry of living tissue to develop biomimetic materials with properties that fit a 
specific design. In a top down approach, intact donor tissue which is already equipped for constructive regeneration, is modified to suit a medical application.

The first biological materials used in medical treatment were made from allogeneic or xenogeneic tissue ${ }^{150}$. Amniotic membrane and cadaver skin were used to cover serious burns as a barrier from infection and external forces. The first kidney transplant was carried out in 1954 using an identical twin donor ${ }^{149,150}$. Since then, the understanding of tissue typing has demonstrated that there are barriers that prevent the transplantation of tissue from one individual to another based on the recognition of immunoglobulins by host cells. Bone marrow transplants, both autologous and allogeneic, were trialled in the $1950 \mathrm{~s}$ and 1960s, and established a cornerstone for what was to become "regenerative medicine" 110 . Unknown at the time, this was the first "stem cell transplant" in which damaged tissue was rebuilt from progenitor cells and their ECM. Tissue engineering products began to emerge on the market; TransCyte in 1997 and Apligraft in 1998 were matrices pre-populated with cells ${ }^{158}$. Cartice was intended for use with autologous cells for cartilage repair. These materials combine matrices and cells in order to rebuild tissue, however the practice of propagating and storing live or cryopreserved cells adds a great cost and risk to these devices.

Acellular or decellularised tissue is easily tolerated by the host and is more easily stored and sterilised than ECM-cell composite materials. As previously mentioned catgut is a natural biomaterial that has been used for centuries. Catgut (an abbreviation of cattle gut) is a cord made from animal intestines, usually sheep or goat (not generally cats) ${ }^{150}$. The method of preparing catgut is essentially a basic "decellularisation" involving the scraping away of external membranes and soaking the remaining tissue in water and potassium hydroxide. Since the material is composed of proteins similar to normal tissue, the body can resorb catgut sutures. Modern catgut sutures can be treated with chromium salts that make them last longer in the body.

In 1979, Chvapil et al. injected different sponges into rabbits to test the toxicity of synthetic materials versus a collagen based sponge. They found that the collagen sponge induced a less destructive immune response than the other polymers tested ${ }^{159}$. There are many 
reasons why a polymer will induce a foreign body reaction, including its surface properties, chemistry and the presence of leachable material ${ }^{148}$. This use of collagen showed that the biological material did not lead to a rejection in rabbits. The body will mount an aggressive response to donor tissue, but this study suggested that collagen alone did not. Another early example of a collagen-based biomaterial was the use of a bovine cornea to replace ocular tissue $e^{2,160}$.

The use of decellularised ECM (dECM) materials began with porcine intestinal submucosa (SIS) as a tissue replacement in $\operatorname{dogs}^{161-164}$. The researchers described the material as an acellular collagen structure. It has since been shown that the material contains a number of other ECM proteins including GAGs and growth factors ${ }^{157}$. In generating SIS, the tissue is treated in such a way that all cells are removed but their ECM remains intact. The absence of cells makes the material tolerant to the recipient's immune system. The porcine material is the basis for a number of medical devices used in soft tissue reconstruction and the first SIS based medical device was cleared by the Food and Drug Administration in 1998.

Decellularised ECMs can be generated from a number of tissues and the yield and quality of the resulting material will depend on many factors. Structural organs, such as muscles and tendons, offer a greater proportion of ECM per cell when compared with organs such as the kidney and liver that have a high number of parenchymal cells relative to the amount of $\mathrm{ECM}^{165-167}$. The submucosa and dermis present a dense layer of ECM within a basement membrane running parallel to a layer of epithelial cells. These structures are well vascularised and rich in GAGs and growth factors. These organs are generally the largest in an animal, meaning that the yield of tissue per animal is higher than other organs.

A large number of ECM based biomaterials are currently available for wound healing and tissue regeneration. They are composed of biocompatible proteins that already possess desirable properties for cell attachment, infiltration and manipulation. Details of some commonly used materials are shown in Table 1-2, modified from Swineheart et al. ${ }^{1}$. Some ECM materials are modified by chemical crosslinking, to improve physical properties for a particular use. 
The success of these materials in surgical applications can be attributed to methods developed for the removal of cellular particles which induce a foreign body response and also to modern sterilisation techniques.

\begin{tabular}{|c|c|c|c|}
\hline le & $\begin{array}{l}\text { TISSUE } \\
\text { SOURCE }\end{array}$ & SPECIES & DEVICE NAME AND MANUFACTURER \\
\hline $1-2$ & DERMIS & HUMAN & ALLODERM (LIFECELL) \\
\hline $\mathrm{dE}$ & & & ALLOMAXTM (BARD DAVOL), GRAFTJACKET \\
\hline $\begin{array}{l}\mathrm{CM} \\
\text { bio }\end{array}$ & PERICARDIUM & BOVINE, EQUINE & $\begin{array}{l}\text { DERMIS BOVINE TISSUEMEND (STRYKER), } \\
\text { VERITAS (SYNOVIS) }\end{array}$ \\
\hline $\begin{array}{l}\text { mat } \\
\text { eria }\end{array}$ & $\begin{array}{l}\text { SMALL } \\
\text { INTESTINAL } \\
\text { SUBMUCOSA }\end{array}$ & PORCINE & $\begin{array}{l}\text { Porcine Surgisis (COOK BIOTECH), } \\
\text { RESTORE (DePuy OrTHOPAEDICS), }\end{array}$ \\
\hline ls & $\begin{array}{l}\text { URINARY } \\
\text { BLADDER } \\
\text { MATRIX }\end{array}$ & BOVINE & MATRISTEM (ACELL) \\
\hline our & $\begin{array}{l}\text { S OVINE } \\
\text { FORESTOMACH }\end{array}$ & OVINE & ENDOFORM (AROA) \\
\hline
\end{tabular}

ECM-based biomaterials can be sourced from a range of tissues and there are a number of factors that will influence the successful engineering of these biomaterials.

\section{Allogeneic or xenogeneic origin}

ECM biomaterials have been made from both human and animal tissue, with the major considerations being safety and $\operatorname{cost}^{165,168,169}$. The use of human and animal tissue poses the risk of viral or prion contamination from donor to recipient. Some patients may refuse the use of human allogeneic grafts on ethical or religious grounds. Xenogeneic ECM materials, especially from cattle are more easily available, bringing the cost of these down, however some patients may object to the use of certain animal species.

Tissue type 
Decellularised tissue has been generated from a large number of tissues and whole organs constituting a diverse array of biochemical and structural features ${ }^{168,169}$. The characteristics of a biomaterial have a large impact on the cost, ease of decellularisation, tensile strength, thickness and elasticity of the final product. Foetal animal tissue, such as amnion and placenta, are more difficult to source than waste or by products such as stomach and intestinal tissue. Additionally, dermal tissue requires an extra processing step during decellularisation because of its keratin layer. Tissue from larger animals such as cows, give a much thicker stronger ECM than the equivalent material isolated from a sheep or pig. Different tissue ECMs will also behave differently in vivo; a study comparing implantation of intestinal and dermal ECM in mice found that the dermal ECM took longer to breakdown ${ }^{170}$.

\section{Age of tissue}

The ability the regenerate slows with age, thus the age of the tissue used to derive the $\mathrm{dECM}$ is a contributing factor to the wound healing capacity of the resultant biomaterial ${ }^{168}$. In a study by Sicari et al. older and younger animals were used to generate different materials $^{171}$. They found that foetal and neonatal ECM contains a greater proportion of immature collagen with fewer crosslinks that is more easily degraded and remodelled. A comparison of constructive remodelling in AECM biomaterials sourced from young and old tissue showed that the implantation of the AECM from young tissue led to greater progenitor cell chemotaxis, a high GAG content and a dominant M2 response culminating in a more effective constructive remodelling response. The difficulty in sourcing material from placental tissue and younger animals must be weighed against the potential benefits.

\subsubsection{Methods of decellularisation}

Decellularised ECM biomaterials are made of proteins that naturally interact with cells in living tissue, as long as the native ECM composition and architecture is retained. The 
method used to remove the molecules that cause an immune response must therefore not interfere too greatly with the structure of the material.

A large number of methods are available to remove cells from a desired tissue $e^{1,165,167-169}$. Ideally, the resulting material will be terminally sterile, immunologically inert and retain biological and structural features of the intact ECM. Decellularisation is a multi-step process that involves:

1. Mechanical treatment of tissue; this step removes unwanted cellular membranes (for instance removal of basement membrane from sub mucosa in the intestinal tract)

2. Removal of cells; generally using ionic solution, detergent or enzymatic digestion and can include EDTA/EGTA to aid cell detachment. Alternatively, freeze thaw cycles can be used

3. Rinsing step; in water or saline to remove cellular debris

4. Chemical sterilisation; using acids such as paracetic acid

5. Rinsing step; to remove chemicals

6. Drying, packaging and terminal sterilisation

Tissues that form the basis for topical dermal biomaterials are best sourced from organs such as pericardium, amnion, bladder, stomach, forestomach and other tissue that form large sheet of ECM. These can then be further manipulated to form materials for a particular purpose. Tissue such as epithelium has a basement membrane component of dense ECM attached to an epithelial layer that can be removed by delamination. Thicker tissue requires a greater number of washes or length of incubations during processing. Other features of the source tissue can make the process more difficult, for example, skin requires a salt wash step to remove the thick keratin layer. Lipid solvents are used to remove fat from some tissues, such as adipose, brain and pancreas. Muscle tissue can be treated with latrunculinB, which disrupts actin and enables the removal of cells. Whole organ decellularisation of lung, liver and heart requires perfusion pumps allowing salts, detergents or enzymes to permeate the entire structure. 
Successful decellularisation is measured by the absence of cells within the tissue and the removal of $\mathrm{DNA}^{168}$. This step is vital as it reduces the immunogenicity of the final products. It has been suggested that the amount of foreign DNA must be less than 50 $\mathrm{ng} / \mathrm{mg}$ of material and DNA fragments should also be smaller than 200 base pairs in size ${ }^{168}$. Another contaminant that must be removed is endotoxin, otherwise the device will induce a pyrogenic response in the recipient. This is particularly important when processing tissue with high levels of natural flora, such as gut and intestine.

The maintenance of key ECM components is very important to the regenerative potential of the device. As such, a successfully decellularised tissue will have collagen structures intact. Comparison of the structure of the tissue before and after decellularisation has shown that there are distinct changes exacerbated by certain decellularisation methods. Freeze thaw cycles, for instance lead to crystal formation and disrupts the collagen $\operatorname{architecture}^{168}$.

The internal ECM structure of a dECM is not the only characteristic that makes it an ideal substrate for cell attachment. Many bioactive molecules are retained within the collagen fibres and GAGs that can be released and utilised by the colonising cells ${ }^{1}$. From attachment zones to cryptic peptides, a mine of bioactives is present in the dECM that wound healing cells can use in the rebuilding of damaged tissue. Other ECM components such as proteoglycans, elastin, fibronectin and growth factors can be measured in the dECM. The procedures of decellularisation must aim to remove cells without destroying underlying, labile secondary components. Harsh decellularisation steps, such as long detergent washes, can reduce the GAG and water content significantly. The presence of growth factors, as measured by ELISA (enzyme-linked immunosorbent assay), is another way to determine whether the process has robbed the ECM of active components.

The "bioactivity" of the material, or its ability to promote wound healing, is more difficult to define. Although many of the components that are bioactive within living tissue may be found in a dECM biomaterial, they may not behave in the same manner after the decellularisation process. Some cell based assays such as the PC12 (pheochromocytoma cell) differentiation and HUVEC (human umbilical vein endothelial cell) migration assays 
may indicate a change in the bioactivity of the material as a result of chemical treatment ${ }^{172,173}$.

Additional processing such as cross-linking can generate materials that are more resilient to degradation and last longer in the body ${ }^{174}$. Some studies have shown that the degree of cross-linking, or other alterations to the native ECM structure are more likely to generate a pro-inflammatory response to the material, as shown by the presence of M1 macrophages $^{168}$.

\subsubsection{The behaviour of ECM materials during tissue regeneration}

Materials used in cutaneous wound healing are hydrated in sterile saline, then placed on a clean, debrided wound or ulcer before the area is loosely covered with a bandage ${ }^{152,172}$. Animal studies with these materials show that the dECM material is infiltrated by FBs, neutrophils and masts cells from the wound edge within days ${ }^{172,173}$. Within weeks, endothelial cells infiltrate the implant and form new microvasculature. The material is completely absorbed in 1 to 6 months, as the wound cells remodel the biomaterial into the host tissue. ECM materials have also been used in abdominal wall repair, bladder and vaginal reconstruction, musculotendinous reconstruction and reconstructive breast surgery amongst others ${ }^{2,128,165,166,175}$.

One of the major benefits of dECMs and other resorbable materials is their ability to promote constructive remodelling rather than a foreign body response. It has been suggested that all biomaterials illicit an immune response after implantation, and the key to success or failure of a device comes down the type and magnitude of that response $\mathrm{e}^{1,3,148,176}$. Historically, biocompatible materials were seen as inert in tissue, however, any sort of injury, cell death or foreign material is met with an immune response. The consequences of that response place materials on a continuum between proinflammatory response and constructive remodelling.

During constructive remodelling, the host is able to completely degrade and replace the material ${ }^{1}$. Similar to normal wound healing, a resorbable biomaterial is infiltrated by 
neutrophils and macrophages, by day 7 the material is completely infiltrated by monocytes. This leads to ECM degradation, the recruitment of stem cells and an appropriate ratio of M1 to M2 macropahges. The foreign body response is an aberrant immune reaction to a material that it cannot properly break down, resulting in dense scar tissue, encapsulation or chronic inflammation. In terms of dECM biomaterials, the immune response to implantation is related to:

1. The efficacy of decellularisation

2. The maintenance of ECM structure

3. Appropriate use of the material

In dECMs, measurable levels of DNA and DAMPS (damage associated molecular patterns) left over from dead cells during decellularisation can enhance a pro-inflammatory response $^{177}$. DAMPS are released by dead cells to warn the surrounding tissue of injury and start of an immune response, using the same toll-like receptor recognition patterns as pathogens. Changes in the ECM structure occur when chemicals are used to crosslink and sterilise the materials. The reason for crosslinking a material is usually to give it greater strength for a particular application. Physical properties of the material must be taken into account, for instance the load bearing and elastic properties of the device ${ }^{165}$. Importantly, the dECM must be applied so that it provides access between healthy tissue and injured tissue so that cells are able to infiltrate and remodel.

Synthetic materials and hydrogels can also be manufactured to act as resorbable scaffolds ${ }^{150}$. Often these materials replicate the ECM in order to promote cell adhesion. If these materials do not illicit a pro-inflammatory immune response they will act as a "scaffold" for healthy cell movement into the injured area. This begs the question: do dECM based biomaterials improve wound healing by acting as a solid support that cells can easily attach to, giving the cells greater access to wound extremities? Alternatively, is the material modifying the behaviour of the wound healing cells and promoting the normal wound healing process?

Extracellular matrix proteins are apposite to the wound healing process; they provide a physical support but they also manipulate the proliferative and migratory behaviour of 
cells. The roles of wound healing cells, ECM proteins and ECM fragments are interdependent. Research into aberrant wound healing and tissue repair seen in aging or diseased tissue has highlighted the balance between the inflammation response and the regeneration response. One reason that is often cited in favour of natural materials is their inherent bioactivity in terms of the promotion of wound healing. Many tissues heal via mechanisms relating to ECM-cell interactions. If $\mathrm{dECM}$ biomaterials retain bioactive molecules in the same way that the ECM does, it is possible that these materials promote wound healing by means of a slow release of bioactive molecules as the matrices are broken down in the body.

\subsubsection{Evidence of bioactive moieties in dECM biomaterials}

\section{Biochemical composition}

One approach to exploring the innate bioactivity of a dECM material is to look at the biochemical composition of the material compared to the source tissue. Ovine forestomach matrix (OFM) is a dECM biomaterial generated from sheep rumen tissue ${ }^{172}$. The tissue is decellularised, dried, cut to size and terminally sterilised giving a flat sheet suitable for use in a number of clinical applications including the treatment of chronic wounds, split thickness skin grafts and rotator cuff repair ${ }^{178-180}$. A biochemical characterisation of the material and the source tissue was carried out by Lun et al. ${ }^{172}$. As shown in Figure 1-6, many important ECM proteins are retained in the decellularised material.

Many ECM components and fragments thereof are responsible for the control of important processes in wound healing such as cell migration, proliferation, and angiogenesis. Growth factors are also vital in the wound healing process and as shown in Figure 1-6, OFM retains $43 \%$ of the FGF2 of the tissue. A number of the growth factors are found in measurable amounts in other materials such as VEGF in porcine $\mathrm{SIS}^{181}$. 


\section{Table 1A}

Major biochemical components of the ovine forestomach and OFM.

\begin{tabular}{lcc}
\hline & $\begin{array}{c}\text { Ovine forestomach } \\
(\mathrm{mg} / \mathrm{g} \pm \mathrm{SE})\end{array}$ & OFM $(\mathrm{mg} / \mathrm{g} \pm \mathrm{SE})$ \\
\hline Total collagen & $142.7 \pm 9.7$ & $821.0 \pm 9.0$ \\
Collagen I & $81.2 \pm 6.6$ & $738.8 \pm 63.3$ \\
Collagen III & $61.3 \pm 0.7$ & $196.8 \pm 6.9$ \\
Collagen IV & $5.4 \pm 1.1$ & $9.7 \pm 2.1$ \\
Elastin & $30.9 \pm 6.4$ & $27.5 \pm 4.5$ \\
Total GAGs & $3.9 \pm 0.1$ & $0.74 \pm 0.01$ \\
Heparan sulfate & $\mathrm{N} . \mathrm{D}$. & $0.31 \pm 0.01$ \\
Hyaluronic acid & $1.95 \pm 0.02$ & $0.4 \pm 0.1$ \\
DNA & $9.0 \pm 0.5$ & $1.7 \pm 0.5$ \\
Lipid & $50.2 \pm 2.9$ & $59.0 \pm 5.2$ \\
\hline
\end{tabular}

Table 1B

Minor biochemical components of the ovine forestomach and OFM.

\begin{tabular}{lcc}
\hline & $\begin{array}{l}\text { Ovine forestomach } \\
(\mu \mathrm{g} / \mathrm{g} \pm \mathrm{SE})\end{array}$ & OFM $(\mu \mathrm{g} / \mathrm{g} \pm \mathrm{SE})$ \\
\hline Fibronectin & $15.30 \pm 1.17$ & $13.67 \pm 1.64$ \\
Laminin & $6.30 \pm 0.24$ & $5.87 \pm 2.16$ \\
FGF2 & $1.70 \pm 1.38$ & $0.74 \pm 0.09$ \\
\hline
\end{tabular}

Errors represent standard error from at least triplicate experiments.

\section{Figure 1-6 The major and minor components of OFM}

taken from Lun et al. ${ }^{132}$.

\section{Cell and animal based models using dECM biomaterials}

Angiogenesis is an essential process in wound healing. Studies have shown that extracts of OFM have a pro-angiogenic effect using the HUVEC scratch assay, rat aortic ring assay and CAM vessel formation ${ }^{173}$. Furthermore, animal models showed that the material promotes the formation of new vessels in an animal wound healing model. The HUVEC migration assay demonstrates the capacity of an extract to generate wound closure on an interrupted monolayer of HUVECs. The aortic ring model shows a change in the number of new vessels growing out of thin slices of aortic tissue embedded in collagen. Similarly, the CAM assay shows branches of new vessels growing on the chorioallantoic membrane in developing chicks. 
PC12 differentiation has been demonstrated using saline extracts of powdered $\mathrm{OFM}^{173}$. In this assay, the number of processes per cell is counted in wells after the extract is added to show any change in the cell phenotype. The results showed that PC12 cells responded to the extracts using FGF2 as a control. It was hypothesised that this was due to the presence of FGF2 in the material. An antibody used to block FGF2 activity in the extract resulted in reduced activity. However, extracts were still able to illicit a response in this assay, suggesting than the FGF2 was not completely blocked by the antibody or that other components in the extract, may be responsible for the activity.

A cryptic peptide isolated from SIS was found to promote osteogenic differentiation in mesenchymal stem cells by Agrawal et al. ${ }^{182}$. Using both in vitro and in vivo models, the researchers found a short peptide derived from collagen which altered the osteogenic differentiation of cells and bone growth in a digit amputation model.

Progenitor cell recruitment as a result of dECM implantation has been demonstrated using a number of in vivo studies, outlined in a review by Swineheart et al. ${ }^{1}$ Importantly, bone marrow derived cells were shown to be recruited to the site of injury after dECM biomaterial implantation ${ }^{183}$. Other progenitors were shown to be recruited to the site of dECM biomaterial implantation including muscle progenitor cells and perivascular cells ${ }^{184}$.

\subsection{Aims of this study}

Biomaterials have been used to promote wound healing and tissue regeneration for many years. Although the mechanisms involved are not fully understood, evidence indicates that the application of a dECM to an area of tissue deficit will promote the healing process ${ }^{1}$. Studies have suggested that biomaterials act as a scaffold, allowing cell infiltration and proliferation around the wound edge, giving the cells necessary traction for wound healing. There is evidence that this improvement in wound healing is not simply due to a physical surface on which the process can occur, but because the material is composed of bioactive molecules that interact with wound healing cells ${ }^{3}$. 
The primary material used in this study is OFM and other ovine-derived ECMs. Previous studies have demonstrated this material as an inducer of angiogenesis and a promoter of wound healing in animal models and clinical studies ${ }^{172,173,178-180}$. Important wound healing cells include: FBs, endothelial cells and mesenchymal stem cells. These cells interact with and breakdown the biomaterial during constructive remodelling. Immune cells are also important during ECM breakdown, as they clear debris and start the organised remodelling process by recruiting neighbouring FBs, stem cells and endothelial progenitor cells. Given that these cells are able to breakdown ECM proteins and release bioactive components from normal tissue, can they mediate the release of bioactive components from a dECM? The specific aims of this study are as follows:

- To describe the interactions between a dECM material and wound healing cells in vitro.

- To determine whether or not a dECM material, or extracts of the material, alter the behaviour of wound healing cells, particularly mesenchymal stem cells, in terms of proliferation and migration.

- To explore the use of cell-mediated processing of the dECM to extract bioactive components from the material. 


\section{Methods}

\subsection{General methods}

A comprehensive list of materials, equipment and manufacturers can be found in Appendix 1 rather than in the text.

\subsubsection{Cell lines}

A list of cell lines and culture conditions is shown in Table 2.1. Unless otherwise stated cells were maintained in DMEM (Dulbecco's modified eagle medium) with FBS during cell propagation.

\begin{tabular}{l|lll} 
CELL & SOURCE & MEDIA & SUPPLEMENTS \\
\hline FB (D551) & ATTC CCL-110 & DMEM & FBS (10\%) \\
$\begin{array}{l}\text { MACROPHAGE } \\
\text { (RAW261) }\end{array}$ & ATTC TIB-71 & DMEM & FBS (5\%) \\
MSC & $\begin{array}{l}\text { ISOLATED FROM } \\
\text { HAEMANGIOMA }\end{array}$ & DMEM & FBS (2 \%) \\
HUVEC & $\begin{array}{l}\text { ISOLATED FROM } \\
\text { HUMAN UMBILICAL }\end{array}$ & RPMI & FBS (10 \%) \\
VEIN & ATTC & DMEM & FCGS (50 $\mu \mathrm{G} / \mathrm{ML})$ \\
EOMA & & & FBS (5 \%)
\end{tabular}

\section{Table 2-1 Cell lines used in this study}

Cells were cultured in a humidified incubator at $37{ }^{\circ} \mathrm{C}$ with $5 \% \mathrm{CO}_{2}$. Human dermal FB cells were obtained from American Type Culture Collection (ATCC, D551- Detroit 551 (foetal dermal fibroblast cell line, identifier CCL-110), and cultured in DMEM supplemented with $100 \mathrm{U} / \mathrm{mL}$ penicillin and $100 \mu \mathrm{g} / \mathrm{mL}$ streptomycin with $10 \%$ FBS. 
Macrophage cells were obtained from ATCC (RAW261, identifier TIB-71) and cultured in DMEM media supplemented with $100 \mathrm{U} / \mathrm{mL}$ penicillin and $100 \mu \mathrm{g} / \mathrm{mL}$ streptomycin with 5 $\%$ FBS.

Human mesenchymal stem cells (MSCs) were isolated from haemangioma tissue at Victoria University of Wellington (Wellington, NZ) by Dr Jun Jia, as previously described $^{185}$. MSCs were able to differentiate down three lineages using osteogenic, adipogenic and chondrogenic differentiation conditions. Images of differentiated and non differentiated MSCs are shown in Appendix 2.

Isolated human umbilical vein endothelial cells (HUVECs) were provided by Dr Sarah Gunningham at Otago University in Christchurch (Christchurch, NZ) and cultured in RPMI media supplemented with $50 \mu \mathrm{g} / \mathrm{mL}$ endothelial cell growth supplemented (ECGS) with $100 \mathrm{U} / \mathrm{mL}$ penicillin and $100 \mu \mathrm{g} / \mathrm{mL}$ streptomycin with $10 \% \mathrm{FBS}$. Culture flasks and plates were treated with $0.5 \%$ gelatine for 1 hour prior to seeding HUVECs.

\subsubsection{Growth factors and supplements}

Platelet derived growth factor (PDGF) was used as a chemo-attractant for FBs and MSCs. The growth factor was diluted in DMEM at a range of $0-25 \mathrm{ng} / \mathrm{mL}$. Vascular Endothelial Growth Factor (VEGF) was diluted in RPMI supplemented with $50 \mu \mathrm{g} / \mathrm{mL}$ ECGS and 1\% FBS at a range of $0-25 \mathrm{ng} / \mathrm{mL}$ as a chemo-attractant for HUVECs.

\subsubsection{Staining reagents}

Stains were diluted in media or buffers, then filtered using a Millipore $0.2 \mu \mathrm{m}$ syringe driven filter. Trypan blue stock solution was prepared in PBS (phosphate buffered saline) at $0.4 \%$ weight per volume (w/v), filtered and stored at room temperature. Trypan blue was added to liquid cell cultures at a ratio of 1:1 before cell counting. Dead cells were identified by dye uptake and live cells excluded trypan blue. 
Neutral red stain was freshly prepared as a $0.008 \%(\mathrm{w} / \mathrm{v})$ solution in cell culture media with $25 \mathrm{mM}$ HEPES buffer and filtered before use. Viable cells were stained by adding an equal volume of neutral red solution to the volume of cell culture media for three hours.

Congo red was prepared as a 100x concentrated stock solution of $10 \mathrm{mM}$ in distilled water $\left(\mathrm{ddH}_{2} \mathrm{O}\right)$ and stored at room temperature. A working solution of Congo red was freshly prepared by adding $100 \mu \mathrm{L}$ of the stock solution to $10 \mathrm{~mL}$ of DMEM media and then filtering, giving a final concentration of $0.1 \mathrm{mM}$.

Toluidine blue solution was prepared in PBS at a final concentration of $0.1 \%(\mathrm{w} / \mathrm{V})$ and stored at room temperature. Alcian Blue stain was prepared as a $1 \%(\mathrm{w} / \mathrm{v})$ solution in $\mathrm{ddH}_{2} \mathrm{O}$ containing $3 \%$ acetic acid volume per volume (v/v). Oil red $\mathrm{O}$ was prepared no more than 2 hours before staining. Oil red O stock was prepared by adding $0.35 \mathrm{~g}$ to 100 $\mathrm{mL}$ of $60 \%$ isopropanol in water $(\mathrm{v} / \mathrm{v})$ to give a $0.35 \%$ solution that was left at room temperature for 10 minutes and then filtered. Alizarin red was prepared in distilled water as a $2 \%$ solution (w/v) then adjusted to a $\mathrm{pH}$ of 4.2. DAPI (4',6-diamidino-2-phenylindole) was used to stain cell nuclei fluorescent blue. This dye was present in ProLong ${ }^{\circledR}$ Gold mounting reagent.

Haematoxylin and Eosin staining was carried out at Hutt Hospital, Lower Hutt according to established protocols. Briefly, deparaffinised slides were stained in Harris' Haematoxylin Solution for 2 minutes then rinsed in running tap water. Slides were dipped in a differentiating solution of $0.3 \%$ (v/v) hydrochloric acid in $70 \%$ ethanol (v/v). Slides were rinsed in running tap water again and then stained in Eosin solution for 2 minutes.

\subsubsection{Fixing methods}

Tissue and decellularised tissue were fixed using $10 \%$ formalin prepared in PBS (v/v). Cultures of cells and OFM were fixed in cold $4 \%$ paraformaldehyde in PBS (for paraffin embedding) or cold methanol acetone solution (1:1) then washed twice in PBS (for cryostat sections). 


\section{Cell monolayers}

Cells grown on coverslips were fixed in cold methanol: acetone solution (1:1), followed by two PBS washes. With the exception of the adipogenic staining of oil red $\mathrm{O}$, in which case coverslips were fixed with $4 \%$ paraformaldehyde (w/v).

\section{Cryostat}

OFM-cell culture samples were placed in a specimen jar containing optimal cutting temperature (OCT) medium, then another layer of OCT was added on top to sandwich the disc before freezing at $-80{ }^{\circ} \mathrm{C}$. To cut sections, the base of the specimen jar was warmed slightly in hand until the specimen and OCT media could be removed with tweezers and placed on the cryostat. Discs were cut into $7 \mu \mathrm{m}$ sections using a Leica CM3050S Cryostat with the following temperature setting: $\mathrm{OT}=-22, \mathrm{CT}=-20$.

\section{Paraffin embedding}

Samples for paraffin embedding were fixed in a solution of $10 \%$ formaldehyde in PBS for two hours before paraffin embedding, sectioning and staining at a core facility (Lower Hutt Hospital, Wellington).

\section{Microscopy}

Minor adjustments to images, such as the correction of brightness and contrasts were made in Adobe Photoshop. Text labels and scale bars were added in Adobe illustrator. Measurements were made using ImageJ software (National Institute of Health).

Live cells were imaged on an inverted CKX41 microscope (Olympus, Japan) and images were captured using a DP12 camera (Olympus, Japan).

Fixed cells and cultures on slides were imaged on an Olympus AX70 photomicroscope using normal light, DAPI or FITC filters. Images were captured using a DP70 CCD camera. 


\subsubsection{BCA protein quantification}

Protein quantification was carried out using a Pierce ${ }^{\mathrm{TM}}$ BCA protein quantification kit using the micro titre plate procedure outlined in the manufacturers' instructions. Bovine serum albumin (BSA) was used as a protein standard, diluted in the same buffer as the protein sample (PBS) using a range of 5 to $250 \mu \mathrm{g} / \mathrm{mL}$. Samples and standards were added to a 96 well plate in triplicate and the kit "working reagent" was added to give a ratio of sample to reagent of $1: 8$. The plate was incubated at $37^{\circ} \mathrm{C}$ for 30 minutes and the absorbance at $562 \mathrm{~nm}$ of each well was read using a Perkin Elmar Enspire plate reader. A standard curve was generated and if $\mathrm{R}^{2}$ was greater than 0.95 the curve was used to calculate the concentration of the samples.

\subsection{Methods for Chapter Three}

Unless otherwise stated, experiments were carried out with OFM; a dECM biomaterial made from ovine rumen tissue that has been decellularised, freeze dried and sterilised according to established procedures ${ }^{172}$. OFM was provided dry and sterile as flat sheets of $4 \times 4 \mathrm{~cm}$ or discs of $16 \mathrm{~mm}$ diameter approximately $1 \mathrm{~mm}$ in thickness.

\subsubsection{Cell seeding and OFM cultures}

\section{Preparation of OFM for cell seeding}

Discs of OFM ( $16 \mathrm{~mm}$ diameter) were used in 24 well cell culture dishes. Flat sheets were hole punched to give circles with a $5 \mathrm{~mm}$ diameter for 96 well plates. These were incubated in $1 \mathrm{~mL}$ or $100 \mu \mathrm{L}$ of DMEM respectively, with or without $10 \% \mathrm{FBS}$ at $37^{\circ} \mathrm{C}$ overnight. To remove excess FBS, discs were then rinsed in DMEM without serum three times before cell seeding. 
Cells were seeded onto $16 \mathrm{~mm}$ diameter $\mathrm{dECM}$ discs at a high density, $(1,000,000$ cells per $\mathrm{mL})$ in a low volume of media $(100 \mu \mathrm{L})$ and allowed 30 minutes to attach before $1 \mathrm{~mL}$ of cell culture media was added to the 24 well cell culture dish. For long-term experiments, half of the DMEM (500 $\mu \mathrm{L})$ was replaced every 48 hours.

\section{Culture cell seeding methods: $5 \mathrm{~mm}$ discs}

Cells were seeded onto 5mm diameter dECM discs at a high density, $(1,000,000$ cells per $\mathrm{mL})$ in a low volume of media $(50 \mu \mathrm{L})$ to give a final number of 50,000 cells per well. Cells were allowed 30 minutes to attach before $100 \mu \mathrm{L}$ of cell culture media was added to the 96 well cell culture dish.

\subsubsection{Attachment and survival assay}

OFM discs $(5 \mathrm{~mm})$ were prepared for cell seeding by conditioning overnight with four solutions:

- Media (DMEM)

- Media with $10 \%$ FBS (DMEM+10 \%FBS)

- Foetal bovine serum (FBS)

- Horse serum (HS)

Discs were placed in $15 \mathrm{~mL}$ tubes containing $5 \mathrm{~mL}$ of each solution and incubated overnight at $37^{\circ} \mathrm{C}$. Discs were then rinsed in DMEM and placed in separate wells of a 96 well plate. 50,000 cells were seeded onto each disc and incubated for three hours at $37{ }^{\circ} \mathrm{C}$. Discs were removed from cell culture dishes using tweezers and gently placed in tubes containing $1 \mathrm{~mL}$ of warmed media. These tubes were gently shaken by inverting the tube before discs were removed and placed into $50 \mu \mathrm{L}$ of media in a new 96 well plate. A working solution of $10 \mathrm{mg} / \mathrm{mL}$ MTT (3-(4,5-dimethylthiazol-2-yl)-2,5-diphenyltetrazolium bromide) was prepared in DMEM and filtered before $50 \mu \mathrm{L}$ was added to each well. This plate was incubated at $37{ }^{\circ} \mathrm{C}$ for one hour. Discs were imaged using an inverted microscope and then MTT crystals were solubilised by pipetting $120 \mu \mathrm{L}$ of MTT 
solubilisation buffer (DMSO: isopropanol, 1:1) into each well. After 5 minutes, $100 \mu \mathrm{L}$ of the liquid from each well was moved to a new 96 well plate and the absorbance of the solution was measured at $550 \mathrm{~nm}$ using a plate reader.

\subsubsection{MSC and FB proliferation and infiltration of OFM}

MSCs and FBs were seeded on $16 \mathrm{~mm}$ OFM discs that had been pre-conditioned with DMEM and 10\% FBS for 24 hours. Cell-OFM cultures were incubated in 24 well plates with $1 \mathrm{~mL}$ of DMEM with $10 \%$ FBS. In each well, half of the media $(500 \mu \mathrm{L})$ was refreshed every 48 hours.

Discs were removed from culture at predetermined time points and gently washed with pre-warmed sterile PBS (500 $\mu \mathrm{L}$ ). A $1 \mathrm{~mL}$ solution of fixative (cold methanol: acetone, 1: 1) was added and the sample frozen at $-20{ }^{\circ} \mathrm{C}$ for 5 minutes. The fixative was then removed and discs washed with PBS $(1 \mathrm{~mL})$. The discs were embedded in OCT embedding media (approximately $3 \mathrm{~mL}$, and frozen at $-80^{\circ} \mathrm{C}$.

Samples were orientated to give a cross section of the disc. In order to estimate the cell number and density on a disc, multiple cross sections were visualised. Sections were taken at intervals of $100 \mu \mathrm{m}$ to give at least five cross sections of the whole disc.

Sections of OFM were placed on Superfrost ${ }^{\mathrm{TM}}$ Slides, dried for 5 minutes at $45{ }^{\circ} \mathrm{C}$ before the OCT was removed by placing slides in a Coplin jar of PBS $(80 \mathrm{~mL})$ for 15 minutes. Slides were stained for 1 minute in $0.1 \%$ toluidine blue to reduce background auto fluorescence, and then rinsed in PBS for 5 minutes. Slides were left to dry and then mounted under cover slips using ProLong Gold® antifade with DAPI.

Slides were visualised using an Olympus AX70 photomicroscope with a DAPI filter. Images of the entire length of a section were captured with an Olympus DP70 CCD camera. 


\subsubsection{Automated image processing and data analysis}

Multiple images were counted using an Image J macro to determine the number of cells per image on a cross section of OFM. Normal tissue and decellularised tissue sections were used as controls to determine the optimal particle sizes for cells using the 10x, 20x and 40x objective lenses of the microscope. The frequency distribution histogram generated in Prism was used to determine the particle size of cells within $25^{\text {th }}$ and $75^{\text {th }}$ percentile of the median. This data is shown in Appendix 3. A copy of the commands in this macro are shown in Table 2-2.

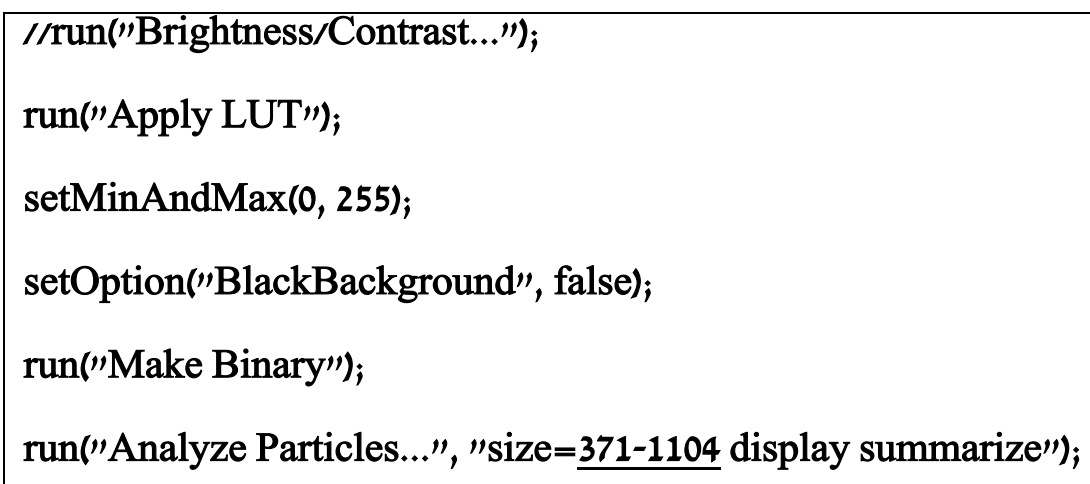

Table 2-2 Automated cell count of cross sections

The commands above were used to manipulate images of OFM cross sections in ImageJ and count the number of cells in each image. The particle size (underlined) was adapted for different magnifications; images captured using 20x magnification measures particles between 371 and 1104 pixels. Images captured at 10x and 40x used a measured a different range of particle sizes as shown in Table 2-3.

\begin{tabular}{ll} 
MAGNIFICATION & PARTICLE SIZE COUNTED \\
\hline $10 \mathrm{X}$ & $75-203$ \\
$20 \mathrm{X}$ & $371-1104$ \\
$40 \mathrm{X}$ & $1345-5321$
\end{tabular}

Table 2-3 ImageJ settings for automated counting of sections 


\subsubsection{Extraction of matrix proteins}

Sheets of dried ECM were cut with a guillotine to give pieces approximately 1 to $2 \mathrm{~mm}$ in diameter. Unless otherwise stated, extractions were carried out with a mass to volume ratio of $1 \mathrm{~g}$ of OFM pieces per $10 \mathrm{~mL}$ of extraction buffer or enzyme solution. After extraction, the solutions were filtered using a syringe driven filter with a $0.2 \mu \mathrm{m}$ pore size and stored at $-20{ }^{\circ} \mathrm{C}$.

\section{Soluble extraction in PBS, culture media and bicarbonate}

Phosphate buffered saline extracts were generated by incubated OFM pieces in PBS at a ratio of $1 \mathrm{~g}$ per $10 \mathrm{~mL}$ at $37^{\circ} \mathrm{C}$, for 16 hours with shaking. The tubes were centrifuged to allow the OFM to settle before the extract was removed, filtered and stored at $-20{ }^{\circ} \mathrm{C}$. The protein concentration was measured using a BCA (section 2.1.5). The PBS extract could be added to a monolayer as third of the final volume of cell culture media in each well.

For cell culture media extractions, OFM was incubated in cell culture media overnight at $37{ }^{\circ} \mathrm{C}$ with shaking before the media was removed, filtered and stored at $-20{ }^{\circ} \mathrm{C}$. Protein concentration of media extracts was not measured by BCA, but estimated from the PBS extracts. Cell culture media extracts could be added directly to a cell monolayer without dilution.

For sodium bicarbonate extracts, OFM was extracted by stirring the pieces at room temperature for 16 hours in an autoclaved solution of sodium bicarbonate $\left(\mathrm{NaHCO}_{3} 0.1 \mathrm{M}\right)$ with a ratio of $10 \mathrm{~g}$ in $500 \mathrm{~mL}$. The solution was filter sterilised and stored. The protein concentration was measured using a BCA. This bicarbonate extract could be added to a monolayer as third of the final volume of cell culture media in each well.

\section{Enzyme digestion of dECM with pepsin and collagenase}

OFM pieces were digested with a solution of pepsin $\left(0.5 \mathrm{mg} / \mathrm{mL}\right.$ pepsin in $\mathrm{ddH}_{2} \mathrm{O}$ with 50 $\mathrm{mM} \mathrm{HCl}$ at $\mathrm{pH}$ 3), using a ratio of $1 \mathrm{~g}$ per $50 \mathrm{~mL}$ pepsin solution. Digestion occurred at 37 ${ }^{\circ} \mathrm{C}$ in a shaking incubator at $100 \mathrm{rpm}$ (revolutions per minute), samples of digested OFM 
were removed at 2 and 4 hours. At the end of the digestion (48 hours), pepsin was inactivated by increasing the $\mathrm{pH}$ of the extracts to $>9.0$ with $1 \mathrm{M} \mathrm{NaOH}$ and incubating at room temperature for 30 minutes. Samples were then neutralised to $\mathrm{pH} 7.0$ with $1 \mathrm{M} \mathrm{HCl}$. Particulate was removed by centrifugation at $300 \mathrm{rpm}$ for 20 minutes, then moving the supernatant to a fresh tube. Pepsin digested OFM could not be filtered in the standard way, so the sterility of the samples was tested by inoculated $500 \mu \mathrm{L}$ on Luria broth agar plates overnight, which showed no growth. The BCA assay was used to determine the protein concentration of each sample. Extracts were stored at $-20^{\circ} \mathrm{C}$ until use.

OFM pieces were digested with a solution of $0.1 \mathrm{mg} / \mathrm{mL}$ collagenase in tris $\mathrm{CaCl}_{2}$ buffer $\left(50 \mathrm{mM}\right.$ tris, $0.36 \mathrm{mM} \mathrm{CaCl}_{2}$ at $\mathrm{pH}$ 7.4) with a ratio of $1 \mathrm{~g}$ OFM per $50 \mathrm{~mL}$ protease solution. Digests were incubated in a shaking incubator at $37{ }^{\circ} \mathrm{C}$ at $100 \mathrm{rpm}$ for 24 hours. The digested solution was filtered and collagenase was denatured with phenylmethanesulfonyl fluoride (PMSF) at a final concentration of $0.1 \mathrm{mM}$ for 1 hour at room temperature. The BCA assay was used to determine the protein concentration of each sample. Extracts were stored at $-20^{\circ} \mathrm{C}$ until use.

\subsubsection{Bioassays: Cell proliferation}

$M T T$

Cells were seeded in 96 well plates at 2000 cells per well in DMEM with $10 \%$ FBS and incubated for 24 hours to settle. Serum starvation was carried out, by replacing the media with DMEM (no FBS) for a further 24 hours. Extracts and controls (media + solvent) were added to the plate and incubated for 48 hours. Extracts and media were removed and $50 \mu \mathrm{L}$ of filtered MTT working solution (10 $\mu \mathrm{g} / \mathrm{mL}$ in DMEM) was added to wells 1 hour. MTT was removed and the intracellular crystals were solubilised in cold dimethyl sulfoxide (DMSO: isopropanol, 1:1) at a final volume of $50 \mu \mathrm{L}$ per well. The absorbance of each well was measured at $550 \mathrm{~nm}$ using a Perkin Almar plate reader. Proliferation was expressed relative to the media control wells. 
Trypan Blue cell count

Cells were seeded at 5000 cells per well in 24 well plates and allowed 24 hours to attach in DMEM with $10 \%$ FBS. Serum starvation was carried, by replacing the media with DMEM (no FBS) for a further 24 hours. Extracts and controls were added for 48 hours. Monolayers were rinsed with PBS and then trypsinised to new $1.5 \mathrm{~mL}$ tubes. Trypan blue (0.4\% in PBS) was added to an equal volume and cells were counted using a cytometer chamber.

\subsubsection{Cell migration assays}

\section{Agarose Plug Migration Assay}

A manuscript describing this methodology can be found in Appendix 5. To generate a chemotactic gradient a layer of agarose was allowed to set above a cell monolayer with a "plug" removed from the centre. A sample mixed with agarose was added to the plug and allowed to set. The procedure is outlines in Figure 2-1.

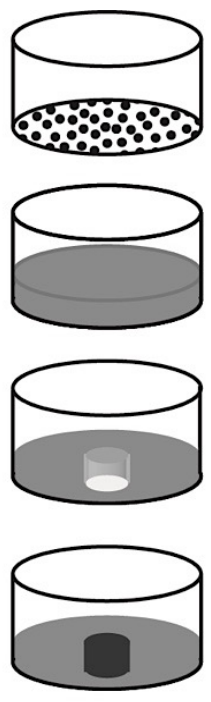

Monolayer of cells established

Molten agarose layer set up and allowed to set.

Central area of cells and agarose removed with biopsy punch

Inner "plug" made of molten agarose mixed with sample, added to centre

\section{Figure 2-1 Chemotactic migration assay set up}

The agarose layer was prepared by mixing equal volumes of cell culture media with $2 \%$ FBS and an autoclaved solution of $3 \%$ low melting temperature agarose (Nuseive ${ }^{\circledR}$ GTG ${ }^{\circledR}$ agarose) in PBS, to give a final concentration of $1.5 \%(\mathrm{w} / \mathrm{v})$ agarose in media. All reagents prepared immediately prior to use and maintained in a molten state at $40^{\circ} \mathrm{C}$ in a water bath. 
Pipette tips and tubes were stored in a $40{ }^{\circ} \mathrm{C}$ before use to prevent rapid cooling and setting of the agarose.

Cells were seeded onto 96 well plates at a density of 10,000 cells per well in DMEM with $10 \%$ FBS and incubated for 24 hours. Serum starvation was carried, by replacing the media with DMEM (no FBS) for a further 24 hours. Media was removed and $100 \mu \mathrm{L}$ of the molten agarose plus media mixture was gently added to each well and allowed to set at room temperature for 20 minutes in a laminar flow hood.

Holes to form the central plug were made in each agarose plug using a $2 \mathrm{~mm}$ diameter biopsy punch attached to a vacuum line to remove a circular section of agarose and underlying cells from the middle of each well. Media $(50 \mu \mathrm{L})$ was added to each well and then removed using the vacuum line to ensure all cells were removed from the centre of the well.

A fresh aliquot of molten agarose plus growth media was prepared containing a known amount of growth factor or sample in a warm tube. The hole in the agarose layer was filled by adding $10 \mu \mathrm{L}$ of the molten solution using a pre-warmed pipette tip. When set, $50 \mu \mathrm{L}$ of media was added to the cover the agarose plug.

Plates containing cells, agarose layer and central plug containing samples and controls were incubated to allow the cells to migrate into the cleared region. The incubation period and the method of image analysis was optimized for each cell line. FBs migrated rapidly into the space and could be counted after 12 hours' incubation with the test sample, while HUVECs and MSCs took longer requiring 24 and 48 hours respectively.

To facilitate visualisation of the cells, viable cells were stained by adding $50 \mu \mathrm{L}$ of neutral red $(0.008 \% \mathrm{w} / \mathrm{v})$ for three hours. For FBs, a large number of cells migrated into the well area in the absence of added growth factor. To improve the sensitivity of the assay when using highly migratory cells, the number of cells in a smaller area of the central well was counted as this requires the cells to migrate a greater distance from the edge of the well.

\section{Scratch Test}


Cells were seeded onto 96 well plates at a density of 20,000 cells per well and incubated for 24 hours, until $90 \%$ confluent). A scratch was made in the monolayer with a pipette tip, perpendicular to a pen line drawn on the underside of the plate before cell seeding. The intersection of the scratch and the pen line was used as the position to image the scratch. Dislodged cells were removed by gentle washing with cell culture media. Media (100 $\mu \mathrm{L})$ containing a dilution series of known chemo-attractants, PDGF (for FBs and MSCs) or VEGF (for HUVECs), was then added to the scratched monolayer. Each well was imaged using a DP12 camera attached to an inverted CKX41 microscope before and after the incubation period. ImageJ was used to determine the denuded area of the monolayer in images of the scratch before and after incubation. The change in area, or "scratch closure" was expressed as a percentage of the original scratch size.

\section{Statistical analysis of bioassays}

The biological read out of the proliferation and migration assays was compared with a media control. A one-way ANOVA test (using $\mathrm{p}<0.05$ ) was use to demonstrate a significant increase in metabolic activity of cells (MTT) and total cell count (Trypan blue stained cells). Similarly, a one-way ANOVA was used to demonstrate a significant increase in "scratch closure" (measured by the percent reduction in the denuded area of the scratch) and the cell count of migrated cells in the chemotaxis assay.

Experiments used different $\mathrm{n}$ values, the MTT assay had a $\mathrm{n}$ of six wells in a 96 well plate (technical replicates). Cell count for proliferation used an $\mathrm{n}$ of three wells of a 24 well plate (technical replicates). Scratch closure was measured as an average of three wells in a 96 well plate and for the chemotaxis assay the cell count was measured in six wells of a 96 well plate. Assays that showed a significant change in cell activity were repeated at least three times (biological replicates). Data in this study is represented as mean values, with error bars calculated by standard error (SE). 


\subsubsection{Preparation of dECM material from other tissue}

Ovine tissue samples were obtained from a meat processing facility (Taylor Preston, Ngaio

Wellington). Samples of ovine rumen, intestine, pericardium were rinsed and the delaminated. Amnion, chorionic and umbilical vein and artery samples were separated from foetal tissue and processed in the same way. Decellularisation of tissue was achieved by rinsing in a series of $1 \mathrm{~L}$ solutions in an orbital shaker at room temperature. ECMs were then flattened and freeze dried overnight on boards before digestion. This process is shown in Figure 2-2.

The following solutions were used for decellularisation:

- TritonX-200 detergent solution - tritonX-200 (1 \% v/v), EDTA (0.1\% w/v) and tris $(0.2 \% \mathrm{w} / \mathrm{v})$ in $\mathrm{ddH}_{2} \mathrm{O}, \mathrm{pH}$ adjusted to 7 .

- SDS solution - $0.1 \% \mathrm{SDS}$ in $\mathrm{ddH}_{2} \mathrm{O}$ (w/v).

- $\quad \mathrm{PBS}$ (for $1 \mathrm{~L}$ ) - $\mathrm{NaCl}(8 \mathrm{~g}), \mathrm{KCl}(0.2 \mathrm{~g}), \mathrm{KH}_{2} \mathrm{PO}_{4}$ (o.2g), $\mathrm{Na}_{2} \mathrm{HPO}_{4}$ (1.15g), adjusted to $\mathrm{pH} 7$ and autoclaved 


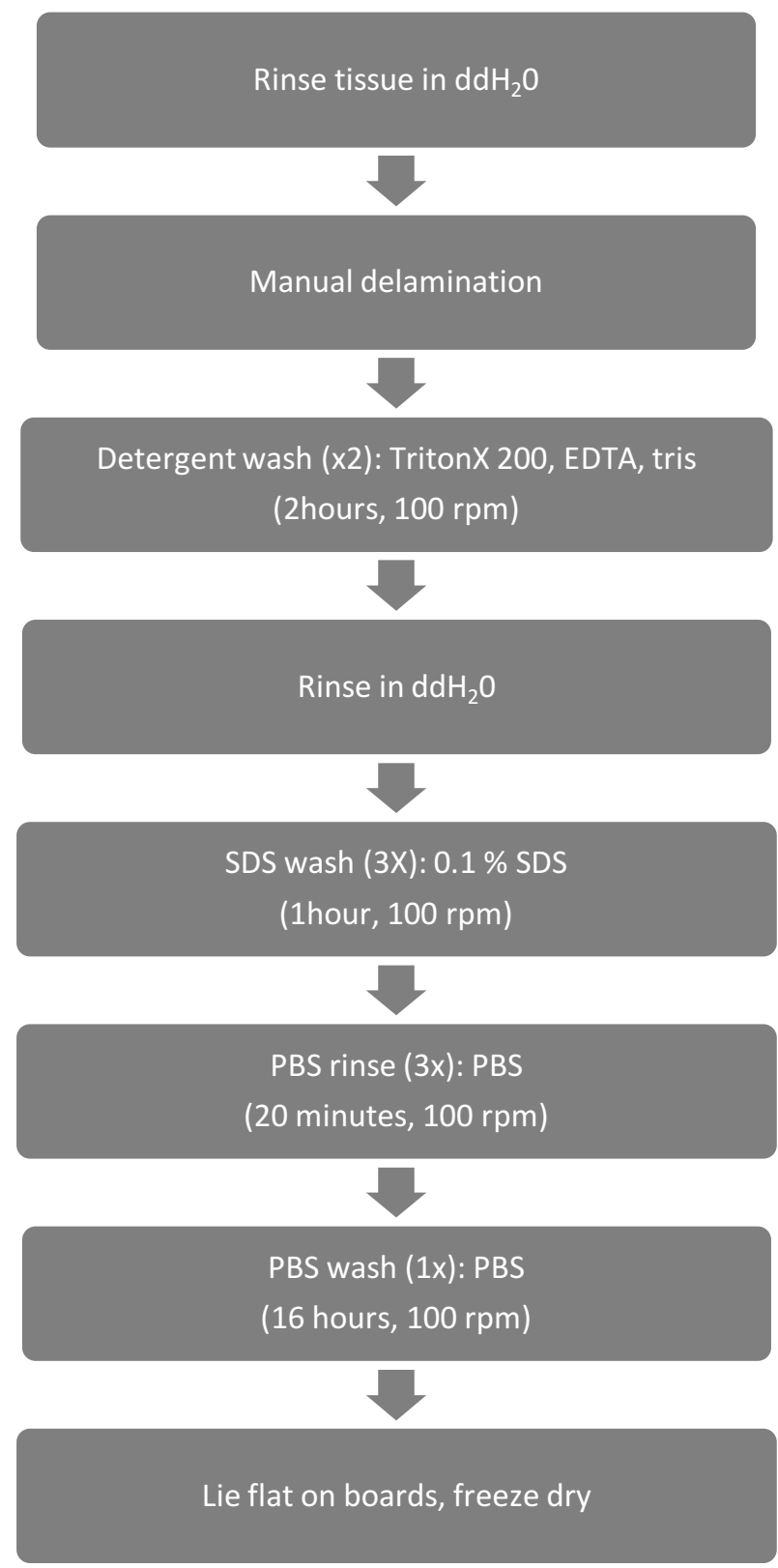

\section{Figure 2-2 Decellularisation process.}

Tissue decellularisation was accomplished by a number of detergent washes. Materials were then rinsed in PBS to remove excess detergent, then freeze dried.

Samples of tissue $\left(1 \mathrm{~cm}^{2}\right)$ were fixed in $10 \%$ formalin in PBS, before and after decellularisation. Samples stained with $\mathrm{H}$ and $\mathrm{E}$ to establish the removal of nuclear bodies within the collagen matrices.

Dermal tissues required an additional salt wash step in order to remove the epidermis from the collagen basement membrane. An additional salt step was added before the detergent washes of $1 \mathrm{M} \mathrm{NaCl}$ for 1 hour after delamination. 
Samples were digested with pepsin for bioactivity screening as described in section 2.2.5.

\subsection{Methods for Chapter Four}

In this chapter ECM proteins were extracted for bioactivity assays using cell-mediated processing of OFM by macrophages and FBs. This required the culture of cells on OFM discs for 48 hours and collection of conditioned media (CM).

\subsubsection{Culture and collection of CM}

OFM discs $(16 \mathrm{~mm})$ were prepared before cell seeding as described in section 2.2 .1 , by preconditioning in DMEM with $5 \%$ FBS. Using a 24 well plate, cultures were setup up in wells containing cells alone, OFM alone and cell-OFM cultures made up in DMEM with 5 $\%$ FBS, as shown in Figure 2-3.

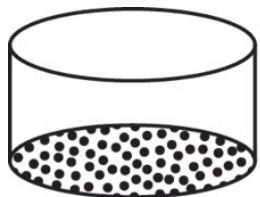

Cell CM (M-CM, D-CM)

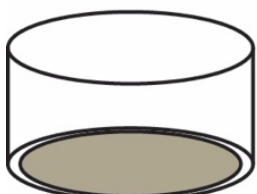

OFM CM (O-CM)

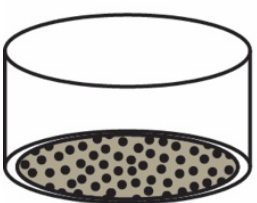

\section{Cell-OFM (MO-CM, DO-CM)}

Figure 2-3 Collection of cell-CM, OFM-CM and cell-OFM-CM

CM was collected after incubating cells, OFM or cell seeded OFM in DMEM for 48 hours.

These extracts are referred to in this study as $\mathrm{CM}$ derived from the following cultures:

- $\mathrm{M}-\mathrm{CM}$ - macrophage conditioned media

- $\quad$ D-CM - FB (D551) conditioned media

- $\mathrm{O}-\mathrm{CM}$ - OFM conditioned media 
- $\mathrm{MO}-\mathrm{CM}$ - macrophage seeded OFM conditioned media

- $\quad$ DO-CM - fibroblast seeded OFM conditioned media

$\mathrm{CM}$ was removed from wells, filtered with $0.22 \mu \mathrm{m}$ pore (to remove any loose cells) and stored at $-20^{\circ} \mathrm{C}$. CM extracts were assayed for bioactivity using proliferation and migration assays described in sections 2.2 .6 and 2.2.7.

\subsubsection{Congo red release and uptake}

Discs were stained by incubating overnight with a solution of $0.1 \mathrm{mM}$ Congo red in phenol red free cell culture media with $10 \%$ FBS. Excess dye was removed by three washed with PBS before seeding the cells onto the stained discs in phenol red free cell culture media with no FBS. The amount of stain released was measured by removing $100 \mu \mathrm{L}$ of media into a 96 well plate and reading the absorbance at $550 \mathrm{~nm}$ using a plate reader.

Cells were gently scraped off the OFM discs using a plastic cell scraper. The disc was removed from the well and the remaining media was gently centrifuged for 2 minutes at $100 \mathrm{rpm}$. The cell pellet was resuspended in fresh media and seeded in a new culture dish. Cells were allowed to attach overnight before wells were gently rinsed in PBS to remove unattached or dead cells and then imaged.

\subsection{Methods for Chapter Five}

\subsubsection{FITC labelling of OFM}

A stock solution of fluorescein isothiocyanate (FITC) was made up at $10 \mathrm{mg} / \mathrm{mL}$ in DMSO and kept as aliquots at $-20^{\circ} \mathrm{C}$ in a light proof container. A fresh solution of $1 \mathrm{mg} / \mathrm{mL}$ FITC was made up fresh in $0.1 \mathrm{M} \mathrm{NaHCO}_{3}, \mathrm{pH}$ 9.3, this was diluted to give a final working concentration of $10 \mu \mathrm{g} / \mathrm{mL}$ in $10 \mathrm{mLs}$.

OFM (16 mm discs or $4 \times 4 \mathrm{~cm}$ squares) were stained by incubation with a $10 \mu \mathrm{g} / \mathrm{mL}$ FITC solution in $\mathrm{NaHCO}_{3}$ in a light proof box on a gentle shaker for 16 hours at $4{ }^{\circ} \mathrm{C}$. Unbound 
FITC was removed by three washes with $10 \mathrm{~mL}$ PBS, for 20 minutes with shaking ( $50 \mathrm{rpm}$ ). OFM was conditioned in cell culture media with 1\% FBS for 16 hours, and washed a further three times in PBS to remove as much FBS as possible in preparation for cell seeding.

\subsubsection{Scale up of CM sample collection}

Macrophage cells were washed in PBS to remove any FBS and spun down to a density of 1 million cells in $1 \mathrm{~mL}$. Cells in $1 \mathrm{~mL}$ were seeded onto $4 \times 4 \mathrm{~cm}$ squares of OFM and incubated in petri dishes for 30 minutes to allow for cell attachment, then $10 \mathrm{~mL}$ of serum free media was added to each dish. The culture was incubated for 48 hours, before CM was collected and filtered using $0.22 \mu \mathrm{m}$ syringe driven filters and stored at $4{ }^{\circ} \mathrm{C}$. Samples are referred to in the text as follows:

- M-CM: macrophage conditioned media

- O-CM: OFM conditioned media

- $\mathrm{O}_{\mathrm{F}}-\mathrm{CM}$ : FITC labelled OFM conditioned media

- MO-CM: macrophage-OFM co culture conditioned media

- $\mathrm{MO}_{\mathrm{F}}-\mathrm{CM}$ : macrophage-FITC labelled OFM co culture conditioned media

Where appropriate, undiluted samples were concentrated using a Centricon $5 \mathrm{kDa}$ cut off centrifugal filter or by spinning in a speed vacuum. Protein concentration was calculated using a BCA as outlined in section 2.1.5.

\subsubsection{Imaging of macrophages after $\mathrm{CM}$ collection}

Macrophages were detached after incubation with FITC labelled OFM in the same way that cells were collected from Congo Red labelled OFM (section 2.3.1). Cell were then imaged under a FITC filter using an inverted microscope with FITC filter. 


\subsubsection{Separation of macrophage and OFM derived proteins by SDS-PAGE}

SDS-PAGE gels were visualised on a fluoroscanner before staining with Coomassie brilliant blue. Stained gels were visualised on a gel scanner.

\section{Sample preparation}

Protein samples were mixed with an equal volume of loading buffer with the reducing agent 2-mercaptoethanol (BME) at $10 \%$. Samples were heated for 10 minutes at $95{ }^{\circ} \mathrm{C}$ and then left on ice before samples were loaded into the gel.

\section{Tris-glycine SDS-PAGE}

Tris-glycine polyacrylamide gels were made up with a $4 \%$ acrylamide stacking gel and a $10 \%$ or $20 \%$ acrylamide resolving gel using a glycine-SDS running buffer. The resolving gel was made up according according to established procedures and poured between glass plate in a BioRad gel assembly system and allowed to set for 1 hour with $1 \mathrm{~mL}$ of distilled water on top of the gel to prevent drying, as per manufacturers' instructions. The stacking gel ( $4 \%$ acrylamide) was poured on top of the stacking gel. The sample loading comb was inserted to allow loading space. The stacking gel was allowed to set for 20 minutes.

The gel was placed in the BioRad gel system with running buffer. A total volume of $15 \mu \mathrm{L}$ of each sample was added per well and $8 \mu \mathrm{L}$ of protein standard ladder (Precision plus protein standards). Glycine gels were run for 1 hour at $100 \mathrm{v}$ after which gels were removed from their glass plates and rinsed in tap water.

\section{Tris-tricine SDS-PAGE}

Tris-tricine polyacrylamide gels were used to resolved smaller proteins according to Schagger et al. ${ }^{186}$. Tricine gels were made with a $4 \%$ acrylamide stacking gel and a $16 \%$ acrylamide resolving gel. Gels were run using a cathode buffer $(100 \mathrm{mM}$ tris, $100 \mathrm{mM}$ tricine, $0.1 \% \mathrm{SDS}(\mathrm{w} / \mathrm{v})$ at $\mathrm{pH} 8.25)$ and an anode buffer $(100 \mathrm{mM}$ tris anode buffer at $\mathrm{pH}$ 8.9). The $16 \%$ resolving gel was made up according according to the established protocol 
and poured between glass plate in a BioRad gel assembly system and allowed to set for 1 hour with $1 \mathrm{~mL}$ of distilled water on top of the gel to prevent drying. The $4 \%$ stacking gel was made up according to established procedures and poured on top of the stacking gel. The sample loading comb was inserted to allow loading space. The stacking gel was allowed to set for 20 minutes.

The gel was placed in the BioRad gel system with running buffers in appropriate chambers. A total volume of $15 \mu \mathrm{L}$ of each sample was added per well and $8 \mu \mathrm{L}$ of protein standard ladder (Precision plus BioRad). Tricine gels were run for two hours at $60 \mathrm{~V}$ on ice after which gels were removed from their glass plates and rinsed in tap water.

\subsection{Gel filtration}

\subsubsection{Sephadex G-25 desalting column}

A desalting column was assembled by rehydrating $1 \mathrm{~g}$ of Sephadex G-25 matrix in $40 \mathrm{~mL}$ of PBS buffer overnight. The matrix was packed into a plastic column to give a $5 \mathrm{~mL}$ column volume which allowed a $0.1 \mathrm{~mL}$ loading volume. Columns were stored at $4{ }^{\circ} \mathrm{C}$ in a solution of $20 \%$ ethanol in $\mathrm{ddH}_{2} \mathrm{O}$ when not in use. Columns were rinsed in $20 \mathrm{~mL}$ PBS before each use.

A sample was added $(0.1 \mathrm{~mL})$ to the top of the column and allowed to sink into the Sephadex matrix, after which $1 \mathrm{~mL}$ volumes of buffer were added sequentially whilst collecting $1 \mathrm{~mL}$ fractions at the other end of the column.

The columns were calibrated with cytochrome $\mathrm{C}$ and bromophenol blue to show the range of fractions that collect protein. The fluorescence of the collected fraction was measured by transferring $50 \mu \mathrm{L}$ volume of each fraction to a plate reader to determine the fractions which contained the protein of interest. 


\subsubsection{DEAE anion exchange}

The buffer for anion exchange used in this study was $10 \mathrm{mM}$ tris $\mathrm{pH} 8.4$ or "DEAE buffer". Diethylaminoethyl (DEAE) beads were swollen in $10 \mathrm{mM}$ tris and $1 \mathrm{~mL}$ of beads were added to a $15 \mathrm{~mL}$ tube using a pipette with the end cut off. Beads were washed three times by filling the tube with $10 \mathrm{mM}$ tris and then centrifuging the beads for ten minutes at 200 rpm, replacing $10 \mathrm{~mL}$ of tris buffer with each wash.

\section{DEAE batch separation}

A $0.5 \mathrm{~mL}$ sample of protein solution was added to $1 \mathrm{~mL}$ of DEAE beads. The tube was vortexed for 2 minutes and then left at room temperature for 5 minutes to allow protein binding. The tube was spun for 2 minutes and the supernatant was collected containing unbound proteins. The same volume of DEAE buffer $(0.5 \mathrm{~mL}$ of $10 \mathrm{mM}$ tris $\mathrm{pH} \mathrm{8.4)}$ was added to the DEAE-sample mixture and the tube was vortexed for 1 minute. The tube was centrifuged for 2 minutes and the supernatant was collected containing wash buffer and unbound proteins. A $0.4 \mathrm{~mL}$ solution of $10 \mathrm{mM}$ tris with $150 \mathrm{mM} \mathrm{NaCl}$ was added to the beads and the tube was vortexed for 2 minutes and then left at room temperature for 5 minutes to allow protein elution. The beads were centrifuged for 2 minutes and the supernatant was collected containing the $150 \mathrm{mM}$ salt eluate. This step was repeated with a solution of $10 \mathrm{mM}$ tris with $400 \mathrm{mM} \mathrm{NaCl}$ to collected the $400 \mathrm{mM}$ salt eluate.

For each collected fraction the fluorescence was measured to give a fluorescent index in relation to the initial sample and relative to the volume of the collected fraction.

The samples were separated by tricine SDS-PAGE as undiluted samples and concentrated to tenth the original volume using a speed vacuum.

\section{DEAE column gradient separation}

A $10 \mathrm{~mL}$ column was packed with DEAE beads, to give a column volume of $5 \mathrm{~mL}$. To prepare the column, two column volumes $(10 \mathrm{~mL})$ of $1 \mathrm{M} \mathrm{NaOH}$ with $0.5 \mathrm{M} \mathrm{NaCl}$ was run through the column. The column was equilibrated with three column volumes $(15 \mathrm{~mL})$ of 
DEAE buffer, followed by a salt wash with $10 \mathrm{~mL}$ of $10 \mathrm{mM}$ tris with $0.5 \mathrm{M} \mathrm{NaCl}$, followed by an acid salt wash of $0.1 \mathrm{M} \mathrm{HCl}$ with $0.5 \mathrm{M} \mathrm{NaCl}$. The column was then rinsed with 4 column volumes $(20 \mathrm{~mL})$ of distilled water and a final $60 \mathrm{~mL}$ of tris buffer until the $\mathrm{pH}$ of the collected buffer returned to $\mathrm{pH} 8.4$.

Samples of $0.5 \mathrm{~mL}$ were loaded into the DEAE column, followed by three column volumes (15 mL) of DEAE buffer. Salt elution was carried out by adding $1 \mathrm{~mL}$ of tris buffer with an increasing salt concentration ranging from $100 \mathrm{mM}$ to $500 \mathrm{mM}$ and a final $1 \mathrm{M}$ step. Fractions were collected in separate $1 \mathrm{~mL}$ tubes as the buffer eluted from the column. The fractions were labelled in the order that they were collected and $0.1 \mathrm{~mL}$ of each was removed to a 96 well plate to measure the fluorescence of the fraction and the protein concentration by BCA.

DEAE column separation was carried out using labelled and unlabelled protein samples side by side. The fractions of interest were pooled to give protein A (unlabelled) and protein B (FITC labelled). The final concentration of the pooled samples was calculated by BCA.

\subsubsection{MSC chemotaxis assay for separated peptide}

Chemotaxis assay was carried out as previously described (section 2.2.7) using the DEAE separated protein A (unlabelled). PDGF was included as a control. Samples were diluted in media using a range of protein concentrations between 1 and $100 \mathrm{ng}$ (final protein concentration).

\subsection{Methods for Chapter Six}

\subsubsection{Sample preparation for MALDI MS}

Gel extraction of proteins

The following solutions were used for MALDI MS preparation. 
- Gel sample wash buffer: 1: 1 acetonitrile (ACN): $100 \mathrm{mM} \mathrm{NH}_{4} \mathrm{HCO}_{3}$

- DTT solution: $10 \mathrm{mM}$ dithiothreitol in $100 \mathrm{mM} \mathrm{NH}_{4} \mathrm{HCO}_{3}$

- IAA solution: $55 \mathrm{mM}$ iodoacetamide (IAA) in $100 \mathrm{mM} \mathrm{NH}_{4} \mathrm{HCO}_{3}$

- Trypsin solution: $1 \mathrm{ng} / \mu \mathrm{L}$ of trypsin in $100 \mathrm{mM} \mathrm{NH}_{4} \mathrm{HCO}_{3}$

- Extraction solution: $0.1 \% \mathrm{TFA}(\mathrm{v} / \mathrm{v})$ in $50 \% \mathrm{ACN}(\mathrm{v} / \mathrm{v})$ in $\mathrm{ddH}_{2} \mathrm{O}$

Protein bands were removed from SDS-PAGE gels by excising the band of interest. A small piece of the gel was also removed from a region with no protein band as a control. Excised gel pieces were cut into smaller cubes no larger than $1 \mathrm{~mm}^{2}$ in an Eppendorf tube using a small spatula. Gel pieces were rinsed by adding $1 \mathrm{~mL}$ distilled water to each tube, vortexing the tube, briefly spinning the contents to the bottom in a bench top centrifuge and then removing the water. Gel pieces were rinsed in a $1 \mathrm{~mL}$ of MALDI wash solution.

Gel pieces were dehydrated in acetonitrile (ACN) by adding enough to completely cover the pieces $(100 \mu \mathrm{L})$ to the tube, removing the liquid and adding fresh $\mathrm{ACN}$ until the pieces turned white. The liquid was removed and the tubes dried for 5 minutes under speed vacuum.

The gel pieces were then swollen in $50 \mu \mathrm{L}$ DTT (dithiothreitol) solution, then incubated for 30 minutes at $56{ }^{\circ} \mathrm{C}$. The tubes were removed from the heating block and the DTT solution replaced by $\mathrm{ACN}$ and the dehydration step was repeated.

The gel pieces were then swollen in $50 \mu \mathrm{L}$ of $55 \mathrm{mM}$ IAA solution and incubated in the dark for 40 minutes at room temperature. The IA solution was replaced with $1 \mathrm{~mL}$ of 100 $\mathrm{mM} \mathrm{NH}_{4} \mathrm{HCO}_{3}$ to rinse the gel pieces, which were then dehydrated in $\mathrm{ACN}$.

Gel pieces were swollen in $50 \mu \mathrm{L}$ of a trypsin solution to give a final mass of $50 \mathrm{ng}$ of trypsin per tube. Tubes were left on ice for 30 minutes after which another $20 \mu \mathrm{L}$ of 100 $\mathrm{mM} \mathrm{NH} \mathrm{NHCO}_{3}$ was added to each tube. Trypsin digestion of proteins occurred within tubes overnight in a water bath set to $37^{\circ} \mathrm{C}$.

The trypsin reaction was halted by adding $5 \mu \mathrm{L}$ of a solution of $5 \%$ TFA (v/v) (trifluoroacetic acid) in distilled water to each tube. The supernatant from the trypsin reaction, containing trypsin digested peptides was collected and moved to a fresh tube. 
Tryptic peptides were extracted from gel pieces by adding $50 \mu \mathrm{L}$ of extraction solution to each tube and vortexing the contents for 30 minutes. Tubes were briefly centrifuged on a bench top centrifuge and the supernatant from each tube collected and combined with the previous trypsin digested peptides from the trypsin reaction. Another $50 \mu \mathrm{L}$ of extraction solution was added to the tubes containing the gel pieces and this process was repeated a further three times. The combined supernatants from these steps were dried in a speed vacuum down to collected down to a volume of less than $20 \mu \mathrm{L}$.

\section{Purification of Tryptic Peptides with ZipTip® salt removal}

The following solutions were used for salt removal:

- Wetting solution: $0.4 \% \mathrm{TFA}(\mathrm{v} / \mathrm{v})$ in $50 \% \mathrm{ACN}(\mathrm{v} / \mathrm{v})$ in $\mathrm{ddH}_{2} \mathrm{O}$

- Equilibration solution: $0.4 \%$ TFA (v/v) in $\mathrm{ddH}_{2} \mathrm{O}$

- Rinsing solution: $0.4 \%$ TFA (v/v) in $\mathrm{ddH}_{2} \mathrm{O}$

- Eluting solution: $0.4 \%$ TFA (v/v) in $70 \% \mathrm{ACN}(\mathrm{v} / \mathrm{v})$ in $\mathrm{ddH}_{2} \mathrm{O}$

ZipTips ${ }^{\circledR}$ (Millipore) were used to remove salts from the trypsin digested peptides. Tips were prepared in wetting solution by gently aspirating and dispensing the solution to waste three times. Tips were equilibrated in equilibration solution by gently aspirating and dispensing the solution to waste three times. Proteins were bound to ZipTips ${ }^{\circledR}$ by repeatedly aspirating and dispensing the peptide solution 20 times. The tips and bound peptides were rinsed using a rinsing solution by gently aspirating and dispensing the solution to waste three times. Peptides were eluted from the ZipTips ${ }^{\circledR}$ by repeatedly aspirating and dispensing $5 \mu \mathrm{L}$ of the eluting solution into a clean tube.

\section{Matrix preparation}

Matrix preparation for MALDI (matrix-assisted laser desorption/ionisation) was carried out by generating a saturated solution of $10 \mathrm{mg} / \mathrm{mL} \alpha$-cyano-4-hydroxycinnamic acid (CHCA) in $0.1 \%$ TFA (v/v), $50 \% \mathrm{ACN}(\mathrm{v} / \mathrm{v})$ in $\mathrm{ddH}_{2} \mathrm{O}$. The solution was briefly spun on a bench top centrifuge and the supernatant was removed to a new tube. Gel extracted, 
trypsin digested, desalted peptides were mixed with matrix solution to give a volume ratio of 1: 9 (peptides: matrix) by mixing $1 \mu \mathrm{L}$ of peptides and $9 \mu \mathrm{L}$ of CHCA matrix. Calibration Mixture 1 for MALDI was mixed with CHCA as a ratio of 1:99 by mixing $1 \mu \mathrm{L}$ of calibration peptides and $99 \mu \mathrm{L}$ of CHCA. The peptide matrix solution was spotted onto a MALDI plate using $2 \mu \mathrm{L}$ per spot.

\subsubsection{MALDI TOF/TOF MS Analysis of Peptides}

MALDI TOF/TOF analysis at Victoria University of Wellington was carried out using an Applied Biosystems/MDS SCIEX 4800 MALDI TOF/TOF analyser. Unknown peptides and calibration mixtures prepared in CHCA matrix were spotted onto a MALDI TOF plate and attached to the magnetic plate holder. The plate was placed on the loading chamber and automatically loaded into the analyser.

The acquisition method was created using the "MS Reflector Positive" operating mode and saved. The signal of the calibration mixture spots was observed by manually selecting a region of the spot using the spectrum viewer.

Two processing methods were created "MS Internal" for calibration spots and "MS default" for unknowns. MS internal processing method was used to calibrate the machinery and update the calibration standards for the acquisition method. MS default was then used to detect peaks in the unknown peptide samples. The spectra generated by individual spots was saved.

\subsubsection{Protein matching with Protein Pilot and MASCOT}

Protein matches were performed using protein pilot software to match peptide masses with known proteins in the protein database downloaded from UniProtKB or NCBI protein databases of Ovis aries or Mus muscularis organisms.

Spectra were opened in protein pilot and search parameters were set to include the following mass modifications: 
- Fixed modification: carbamidomethyl of cysteinc by ioadoacetamide

- Variable modifications: oxidation of methionine

Searches were carried out using the following databases:

- NCBI eukaryote nonredundant protein database (NCBInr, 34397589 sequences, 20131128)

- UniprotKB Mus muscularis protein database (16714 sequences, 20141106)

- UniprotKB Ovis aries protein database ( 31121 sequences,20131204)

\subsubsection{Sample preparation for MALDI and ESI at APAF}

Samples were sent to the Australian Proteome Analysis Facility (APAF) for analysis by MALDI and ESI (electrospray ionisation) MS.

Samples were sent for analysis as "in solution" samples and "gel excised" peptides. In solution samples were collected as described in section 2.3.1 and desalted using a Sephadex G-25 column (as described in section 2.5.1). $20 \mu \mathrm{L}$ of the protein solution was dried to a pellet using a speed vacuum to give a solid containing $2 \mu \mathrm{g}$ total protein. Gel excised samples were prepared by removing the band of interest from an SDS-PAGE gel. MALDI and ESI MS reports sent by APAF are attached as Appendix 4.

\subsubsection{Theoretical digestion of protein candidate using MEROPS database}

The MEROPS database (http: //merops.sanger.ac.uk/) was used to search for known MMP substrates within the protein of interest decorin $(\mathrm{DCN})^{187}$. The sequence of the amino acids in decorin (accession number AAF00585) obtained from NCBI was used to map theoretical protease cleavage sites within the protein. Peptide masses were calculated based on the amino acid sequence using the online tool from the peptide synthetics website (http://www.peptidesynthetics.co.uk) so the map of fragments could be drawn to scale. These were compared with peptides identified by ESI MS analysis of conditioned media using peptides generate by trypsin and GluC digestion of the sample. 


\section{Dynamic reciprocity between wound healing cells and the extracellular}

\section{matrix}

\subsection{Summary}

Extracellular matrix proteins such as collagen, laminin, fibronectin and GAGs are an essential part of the wound healing process; they act as a physical support but they also manipulate the proliferative and migratory behaviour of cells ${ }^{63}$. In this chapter dECMs and dECM-derived moieties are explored in relation to their interaction with relevant wound healing cells in vitro. Firstly, a dECM material is used as a substrate for cell proliferation and infiltration. Secondly, extracts of the material are investigated as a source of wound healing cell mitogens and motogens.

\subsection{Introduction}

For hundreds of years, mankind's capacity to self-heal has been aided by foreign materials that interact with the healing tissue ${ }^{150}$. ECM based materials, specifically tissue that has been decellularised leaving the rudimentary ECM components, act as a scaffold for wound healing cells as they migrate, proliferate and synthesise a new ECM during wound healing. One such material is "ovine forestomach matrix", which is derived from sheep rumen tissue $^{172}$. 


\subsubsection{Modelling an ECM culture of wound healing cells in vitro}

ECM-derived products can be used as scaffolds for in vitro cell culture models. Hydrogels and acellular matrices such as Matrigel ${ }^{\circledR}$ have been used to support cells in three dimensional cultures for a number of applications including drug screening and angiogenesis $^{188-190}$. Many researchers are promoting the use of three dimensional culture models as a means to more accurately model cell behaviour in vitro. Three dimensional models are more reminiscent of living tissue than cell monolayers because they encompass cell-matrix interactions that influence factors such as drug bioavailability and cell signalling.

DECM biomaterials have not been explored in this capacity, despite ample evidence that they interact with a number of cells types during wound healing and tissue generation. To use a material such as OFM in this way, cells would need to be able to adhere to, proliferate on and infiltrate the material. An OFM-cell culture could be used as a model system for FB matrix remodelling, stem cell differentiation and vessel formation by endothelial cells. In this chapter the feasibility of using OFM as a support for the three dimensional cell culture of wound healing cells is explored.

\subsubsection{Bioactive components in dECMs}

Previous studies have shown that dECMs are "bioactive" in relation to the constructive remodelling process of wound healing and tissue repair. This topic has been explored in relation to the biochemical composition of the materials ${ }^{172,181}$, cell based assays ${ }^{173,182}$ and human and animal studies ${ }^{161,179,184}$ using dECM materials. In this study, the relationship between $\mathrm{dECMs}$ and wound healing is explored, with an emphasis on mesenchymal stem cells.

During wound healing, proliferation and migration are two processes that are vital and highly regulated in a number of cell types. In this chapter, dECMs are examined for the 
presence of mitogens and motogens, components that promote cell proliferation and motility respectively.

\section{Other materials used as dECMs}

Extracellular matrix biomaterials can be generated from a large number of tissues. In different tissues, cells control the biochemical make up of their ECM for the particular functions of that tissue. Since different tissues display different regenerative capacities, dECMs from different tissues may vary in their bioactivity. The primary biomaterial used in this study, OFM, has been subject to an in-depth analysis of its biochemical makeup, biophysical properties, angiogenic properties and clinical use ${ }^{172,173,178-180}$. For this study, dECM materials were generated from other ovine tissue including intestine, pericardium, amnion, dermis, stomach and placental vein and artery tissue.

\section{Cells used in OFM cultures}

During wound healing, cells, proteins and proteases are responsible for concerting the overlapping processes. Many cell types are activated or switched from a "resting" state to a "wound healing" state when subject to injury ${ }^{63}$. For cells involved in wound healing, this requires an increase in the number of cells at the wound site by migration, chemotaxis and invasion followed by proliferation and protein expression. The primary cells responsible for the synthesis of ECM proteins are myofibroblasts, derived from FBs and MSCs. Endothelial cells and endothelial progenitor cells generate a network of new blood vessels to provide oxygen to the healing tissue.

FBs and myofibroblasts in living tissue are responsible for maintaining the integrity of the matrix and cause contractions of the collagen fibres ${ }^{52,54}$. They are also the primary matrix building cells in most ECMs, expressing procollagen strands that can then assemble into fibres. In wound healing, FBs switch to an active state during which they are highly proliferative and are actively recruited to the site of injury where they begin collagen synthesis. 
Meirelles et al. define MSCs as a heterogeneous population of multipotent mesenchymal stromal cells that will, in theory, give rise to all connective tissue ${ }^{107}$. In practice, they can differentiate into bone, cartilage and adipose tissue in vitro. MSCs in wound healing migrate from bone marrow and surrounding tissue, giving rise to keratinocytes, endothelial cells, macrophages and pericytes. The MSCs used in this study represent a readily available source of adult mesenchymal stem cells. They are a heterogeneous mixture of bone marrow derived cells; including endothelial progenitors, alpha-smooth muscle actin expressing progenitors, and fibroblast-like cells. They express mesenchymal markers and are able to differentiate into multiple lineages making them an effective model line ${ }^{185}$. Endothelial-ECM three dimensional cultures are a useful way to model angiogenesis and tube formation. Endothelial cells are often grown on an ECM-base substrate such as gelatine, collagen or Matrigel ${ }^{\circledR}$, this allows the researcher to observe endothelial cell migration, organisation and even tube formation under the right conditions ${ }^{191,192}$. These cells model the capillary-mobilised and blood circulating endothelial progenitors that play a role in angiogenesis and vascularisation of granulation tissue in the healing wound.

\subsubsection{Wound healing assays}

\section{Proliferation assays}

Many of the cells involved in wound healing are activated by tissue injury to migrate to the site of injury and proliferate. Proliferation assays using tetrazolium dyes, are a way to measure the effect a sample may have on cell proliferation. MTT (4,5-dimethlthiazol) is a yellow salt that is reduced to purple formazan by oxidoreductase enzymes in living cells. In a growth analysis, cells are incubated with extracts to an endpoint, then incubated with MTT. Purple formazan crystals form in viable cells, the crystals are solubilised and the absorbance of the cell culture media is measured to indicate the number of cells in each well. Some extract may alter the activity of cellular nitro reductase enzymes, so this method is often validated with another proliferation assay. 


\section{Migration assays}

Cell migration is a vital process in wound healing. There are a variety of cytokines and matrikines that recruit cells to the site of wound healing and measuring these in vitro does not take into account the many intricacies of dynamic reciprocity that have been discussed. Cells respond to interruptions in cell-to-cell and cell-to-matrix interactions by increasing their motility. The scratch assay measures the closure of a gap in a cell monolayer. Cells are cultured to a high confluence, then the monolayer is interrupted with a "scratch" and cells at the leading edge migrate inwards to seal the gap. This assay can be used to identify materials that promote or inhibit chemokinesis.

A major disadvantage of this assay is that it measures cell movement but does not account for the direction of movement. In chemotaxis assays, cells respond to a chemotactic gradient of an extract. The ability for cells to move from a low to a high concentration of a chemokine is essential in wound healing. Cells are able to sense their way to the site of injury because cytokines bind to specific receptors that signal the required direction of movement to the cytoskeleton. To test the chemokinetic activity of an extract, an assay must generate a low and high concentration of a chemokine and measure the number of cells that migrate from the former to the later.

\subsection{Aims}

This chapter will focus on how dECMs and AECM-derived components interact with important cells in the wound healing process in vitro.

I. Firstly, in vivo models show that FBs and endothelial cells infiltrate dECMs during constructive remodelling. Do wound healing cells attach to, proliferate on and infiltrate the OFM material in vitro as they do in living tissue? If so, can dECMs be used for cell research as well as wound healing applications?

II. Secondly, are there proteins, peptides or other molecules derived from OFM that interact with wound healing cells? Specifically, do OFM components promote wound healing cell proliferation and migration? 
III. Finally, if there is an effect on cell proliferation and migration from OFM components, is that mirrored in dECMs sourced from other ovine tissue?

\subsection{Results}

\subsubsection{Cell attachment on OFM in vitro}

Ovine forestomach matrix (OFM) is a freeze dried dECM material, therefore it must be rehydrated before use. For cell culture, OFM discs are conditioned in cell culture media for 12 hours, before cells will adhere to the material in vitro. In this experiment, FBs were seeded on OFM after the discs had been conditioned in PBS, cell culture media (DMEM), DMEM with $10 \%$ FBS, FBS alone and horse serum (HS) alone. After gently rinsing away unattached cells, a solution of MTT was added to the culture. Live FBs that had attached to the discs, developed purple formazan crystals, making them visible under an inverted microscope. Cell attachment was quantified by dissolving the crystals and collecting the culture media to measure the absorbance.

Figure 3-1 shows images of OFM discs with FBs attached after the discs have been conditioned in different ways; PBS, DMEM, HS, FBS, DMEM and $10 \%$ FBS. Below this, the average absorbance of each culture after MTT dissolution is shown, normalised to the absorbance in wells with an OFM disc with no cells $(n=3)$. The images show that cells are able to attach to the OFM, but greater attachment is achieved if discs are pre-conditioned with media and FBS, FBS and HS. The absorbance of the culture media shows that a significantly higher concentration of formazan is dissolved from cultures that have been conditioned in DMEM with $10 \%$ FBS and FBS than media alone. This indicates that there are a greater number of cells attached to these cultures. The difference between cell attachment in $10 \%$ and $100 \%$ FBS pre-conditioning is not as great as the difference between $0 \%$ (DMEM alone) and $10 \%$ FBS pre-conditioning of the OFM discs.

72 Dynamic reciprocity between wound healing cells and the extracellular matrix 

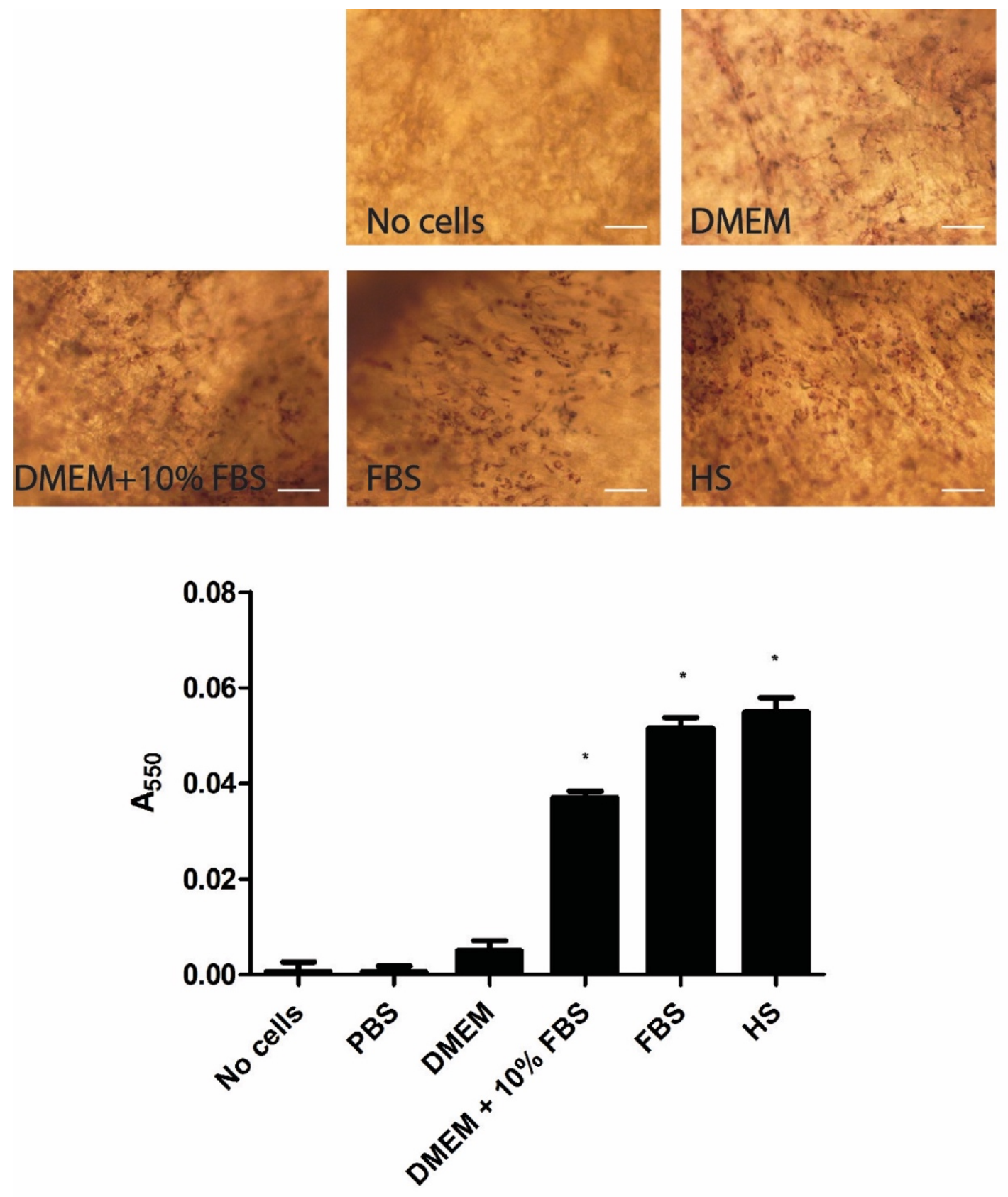

Figure 3-1 FB attachment to conditioned discs of OFM in vitro

Discs were conditioned in PBS, DMEM, DMEM with $10 \%$ FBS, FBS and HS before cell seeding. Cells attachment was quantified by MTT. Representative images of cellseeded discs are shown in images. Scale bar (white) is $100 \mu \mathrm{m}$. The discs conditioned with DMEM + $10 \%$ FBS, FBS and HS showed significantly higher cell attachment as measured by MTT, compared with discs conditioned with PBS and DMEM. Quantifications are averages of three independent cultures, error bars indicating SE. 
Statistical significance $(\mathrm{p}<0.05)$ was calculates using ANOVA and Dunnetts multiple comparison test.

\subsubsection{Proliferation and infiltration into OFM}

In order to visualise cell infiltration on OFM, cells were seeded onto one side of discs preconditioned in DMEM with $10 \%$ FBS. Cell-OFM cultures were incubated, with $50 \%$ of the culture media refreshed every 48 hours. The number of cells and level of infiltration was observed following a gentle rinse to remove unattached or dead cells and fixation of the whole discs. Discs were mounted and sectioned giving five evenly spaced cross sections spanning the diameter of the discs.
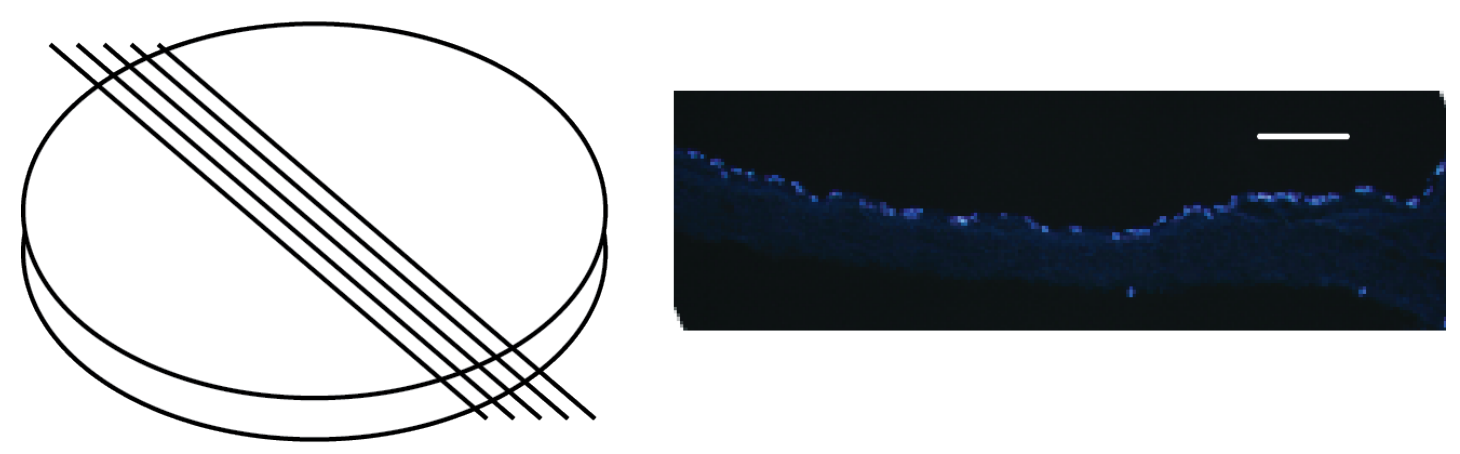

Figure 3-2 Cross section of cell-OFM culture.

Schematic of OFM disc cross sections and example of cross sectional image of OFM and cell culture. OFM discs are $16 \mathrm{~mm}$ in diameter and approximately $100 \mu \mathrm{m}$ thick. Five sections $(7 \mu \mathrm{m}$ thick) were cut across the centre of the disc. A cross sectional image of OFM is shown after cell seeding, showing nuclei stained blue by DAPI on the seeded surface. Scale bar (white) is $100 \mu \mathrm{m}$.

As shown in Figure 3-2 sections of OFM-cell cultures collected this way give an indication of the number of cells on each disc and whether they remain on the seeded surface or infiltrate the material. As the image of a cross section shows, the cells distributed across 
the section and do not infiltrate the material. This behaviour was observed using FBs, endothelial cells (HUVECs), EOMAs and MSCs (data not shown).

\section{FB non-infiltration on OFM}

A number of modifications were made to the OFM FB culture, including the seeding density and incubation time. In addition, OFM discs that had been perforated were used. None of these conditions resulted in a change in the level of cell infiltration of the material. As shown in Figure 3-3, cells remained on the seeded side of the disc after 1 month in culture.

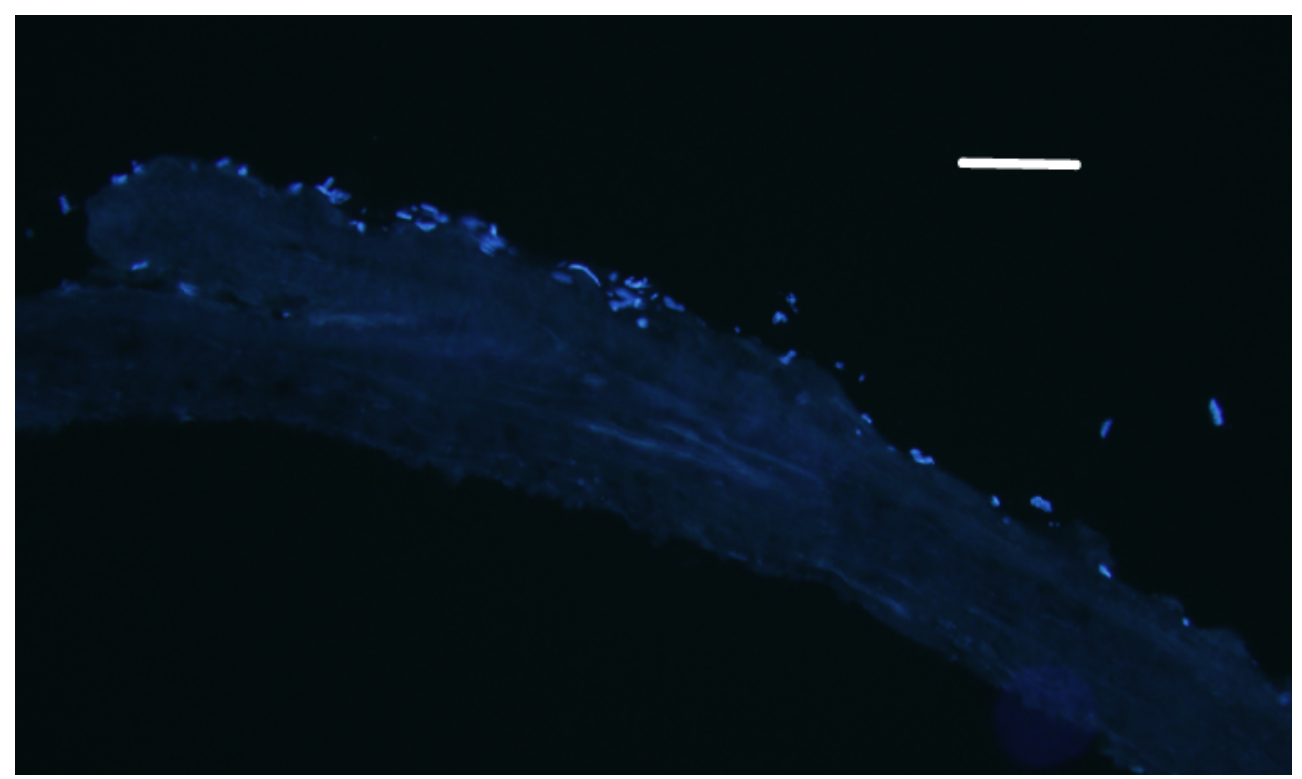

Figure 3-3 FB non-infiltration of OFM

Cells seeded on OFM discs and cultured for 1 month did not lead to a change in infiltration of the OFM material. Scale bar (white) is $100 \mu \mathrm{m}$

\section{Proliferation of MSC in OFM co culture}

Cell proliferation in the culture was measured by counting the number of cells attached to each cross section. A total number of cells on three sections per disc were counted and an average of cells on three separate cultures were calculated for each time point. ImageJ software was used to automate the counting of cells grown on OFM discs (Appendix 3). Figure 3-4 shows the number of cells on each section and the number of hours in culture. 


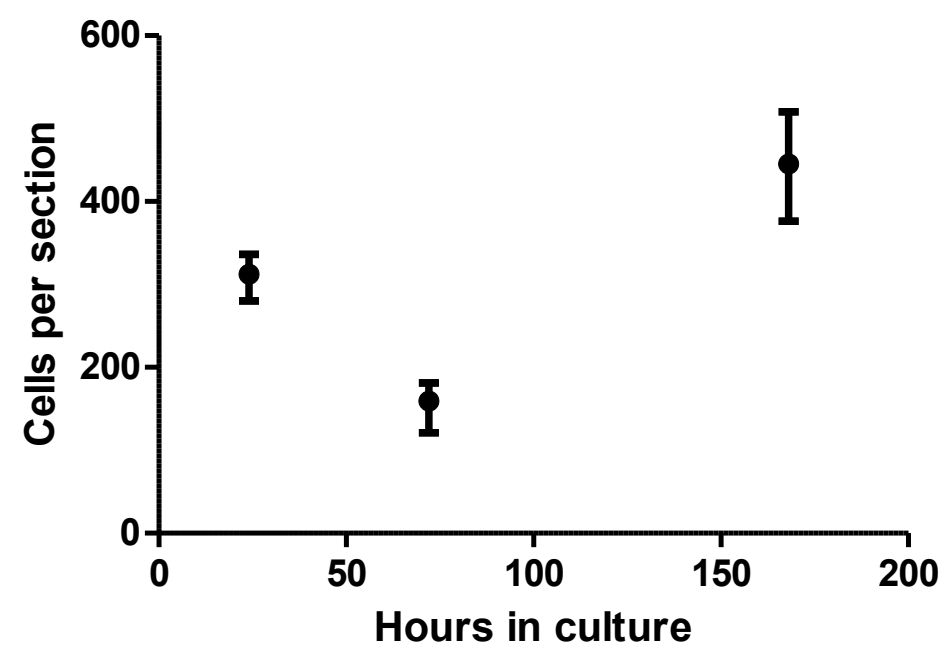

Figure 3-4 Proliferation of MSCs in OFM culture

MSCs were seeded and grown on OFM for up to 7 days (172 hours). The average number of cells in three sections from three separate discs was calculated to determine cell proliferation in culture.

Figure 3-4 shows that the number of MSCs seeded onto OFM initially goes down in the first two days of culture and then rises to greater than 400 cells per section after a week in culture.

\subsubsection{Bioactivity of OFM proteins in solution: cell proliferation}

OFM extracts were collected after incubating the material in PBS, cell culture media and sodium bicarbonate. OFM digests were collected using proteolytic enzyme pepsin. These samples were tested on MSC and FB cell monolayers to assess bioactivity in relation to in vitro cell proliferation using an MTT assay. The change in absorbance after a 48-hour incubation with each extract or digest was expressed relative to the media control. The soluble extracts of OFM using PBS, DMEM and bicarbonate led to no significant increase in cell proliferation by MTT (this data is not shown). Digested extracts led to an increase in cell proliferation for MSCs but not FBs.

Figure 3-5 (A) shows the change in absorbance of MSC monolayers after incubation with pepsin digested dECM proteins. Extracts of pepsin digested OFM were prepared using

76 Dynamic reciprocity between wound healing cells and the extracellular matrix 
digestion times of 2, 4 and 48 hours; samples A, B and C respectively. All samples were normalised to a final concentration of $300 \mu \mathrm{g} / \mathrm{mL}$ per well, with six replicate wells. This data is representative of three separate experiments. There is a significantly higher number of cells in wells incubated with pepsin-digested OFM relative to media controls. Sample $\mathrm{C}$, which had been digested for the longest, gave the largest increase in MTT metabolism compared with the media control. The experiment was repeated using sample $\mathrm{C}$ in a cell count, to confirm the MTT result (Figure 3-5 (B). The change in cell number is significantly greater than the number of cells incubated in media alone using t-test $(p<0.05)$.
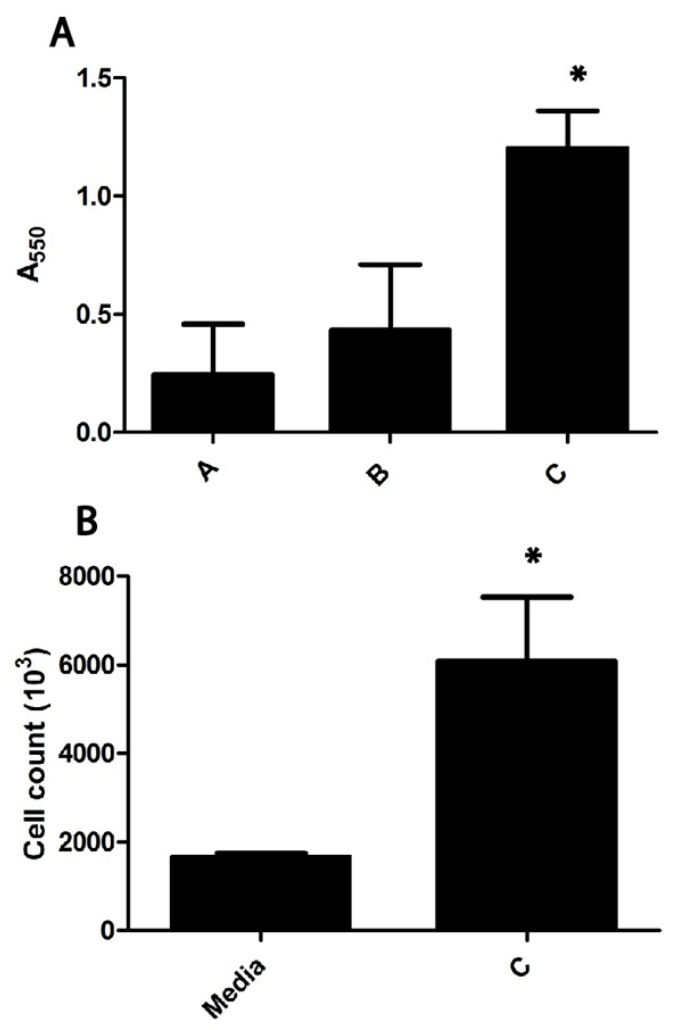

Figure 3-5 Proliferation assays with MSCs

(A) MSC monolayers were incubated with samples A B and C, OFM digested with pepsin for 2, 4 and 48 hours respectively. Samples were diluted with PBS to a final protein concentration of $300 \mu \mathrm{g} / \mathrm{mL}$ per well. After a 48-hour incubation the absorbance of each well was measured using the MTT assay. The graph above shows the absorbance, normalised to a control of media with PBS. Error bars indicate the SE 
between six wells. This data is a representative result of three independent experiments. (B) The change in cell number between wells with a PBS control and sample C at a final concentration of $100 \mu \mathrm{g} / \mathrm{mL}$. Error bars represent the SE between three wells. Significant increase in proliferation compared with media controls (*) was calculated by t-test $(\mathrm{p}<0.05)$.

\section{Summary of proliferation activity}

Table 3-1 shows that using these methods, the only significant change in cell proliferation was observed using MSCs and pepsin digested OFM. The extracts of OFM collected by enzyme digestion had a significant effect on MSCs but not FB proliferation. Extracts collected by solvent extraction in media, PBS or sodium bicarbonate, did not lead to a change in cell proliferation. Importantly, the FBs used in this assay cannot be cultured in very low serum cultures, the addition of FBS in these proliferation assays may have supressed the activity of extracts cultured from these cells. For this reason, HUVECs were not tested for proliferation, as they must be cultured in media supplemented with FBS and ECGS.

\begin{tabular}{|c|c|c|c|}
\hline & FB & MSC & HUVEC \\
\hline PEPSIN DIGEST & $\begin{array}{l}\text { NO CHANGE } \\
\text { (DATA NOT SHOWN) }\end{array}$ & $\begin{array}{l}\text { SIGNIFICANT INCREASE IN } \\
\text { PROLIFERATION AS TESTED } \\
\text { BY CELL COUNT AND MTT } \\
\text { (FIG 3-5) }\end{array}$ & No DATA \\
\hline PBS EXTRACT & $\begin{array}{l}\text { NO CHANGE } \\
\text { (DATA NOT SHOWN) }\end{array}$ & $\begin{array}{l}\text { No CHANGE } \\
\text { (DATA NOT SHOWN) }\end{array}$ & \\
\hline $\begin{array}{l}\text { SODIUM } \\
\text { BICARBONATE } \\
\text { EXTRACT }\end{array}$ & $\begin{array}{l}\text { NO CHANGE } \\
\text { (DATA NOT SHOWN) }\end{array}$ & $\begin{array}{l}\text { NO CHANGE } \\
\text { (DATA NOT SHOWN) }\end{array}$ & \\
\hline
\end{tabular}

Table 3-1 Summary of proliferation activity of OFM extracts

3.4.4 Bioactivity of OFM proteins in solution: cell migration

Scratch Assays

78 Dynamic reciprocity between wound healing cells and the extracellular matrix 
In order to test the digests for molecules that affect cell migration, scratch assays were carried out with monolayers of HUVECs and MSCs. Solutions of pepsin and collagenase digested OFM were incubated with a scratched monolayer and imaged before and after incubation to calculate the scratch closure.

For HUVEC scratch assays, a VEGF positive control and a PBS (solvent) negative control was included. Figure 3-6 show images of HUVEC monolayers before and after treatment with (A) VEGF and (B) PBS controls. ImageJ software was used to generate outlines of the scratch area before and after treatment, the change in scratch area could be measured to calculate scratch closure. In Figure 3-6 (C) the calculated scratch closure was significantly greater in wells containing VEGF, pepsin digested OFM and collagenase digested OFM, compared with a PBS control.
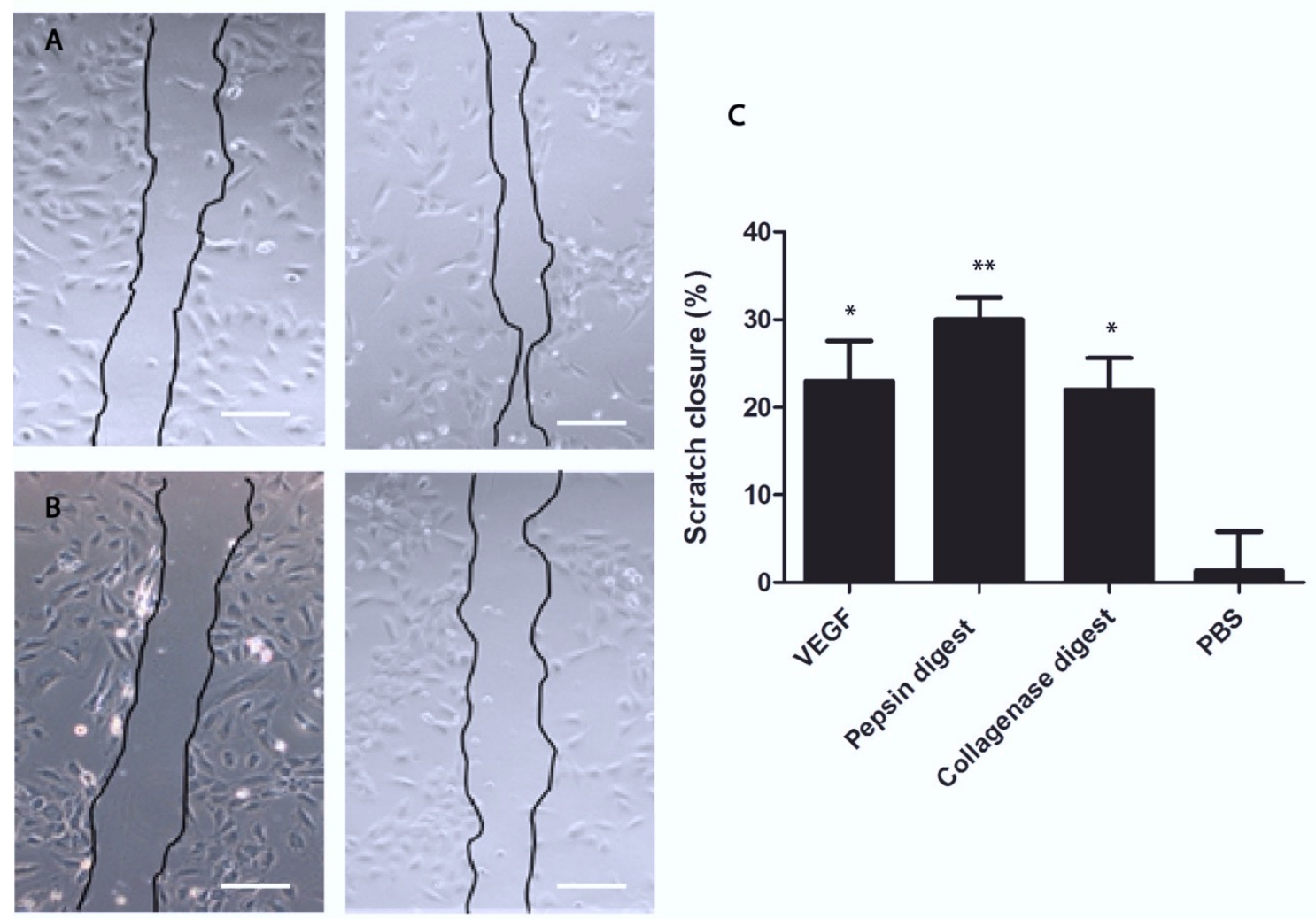

Figure 3-6 HUVEC scratch assays

HUVEC monolayers were scratched, then incubated for 6 hours with OFM digests at 100 
$\mu \mathrm{g} / \mathrm{mL}$ using pepsin and collagenase. Photomicrographs of the scratched area are shown in (A) VEGF control 0 and 6 hours and (B) media control at 0 and 6 hours. Scale bar (white) is $50 \mu \mathrm{m}$. (C) Migration was expressed as the percent scratch closure for VEGF, pepsin and collagenase digested OFM extracts and a PBS control. Error bars indicate SE from triplicate wells. Samples with significant change in scratch closure in relation to PBS control (*) and (**) were calculated by one-way ANOVA $\left({ }^{*} \mathrm{p}<0.05\right.$ and ${ }^{* *} \mathrm{p}<0.017$.

For MSC scratch assays, a VEGF control and a PBS (solvent) control was included. Figure 3-7 show images of MSC monolayers before and after treatment with (A) VEGF and (B) PBS controls. ImageJ software was used to generate outlines of the scratch area before and after treatment, the change in scratch area could be measured to calculate scratch closure. In Figure 3-7 (C) the calculated scratch closure was significantly greater in wells containing VEGF, pepsin digested OFM and collagenase digested OFM, compared with a PBS control.
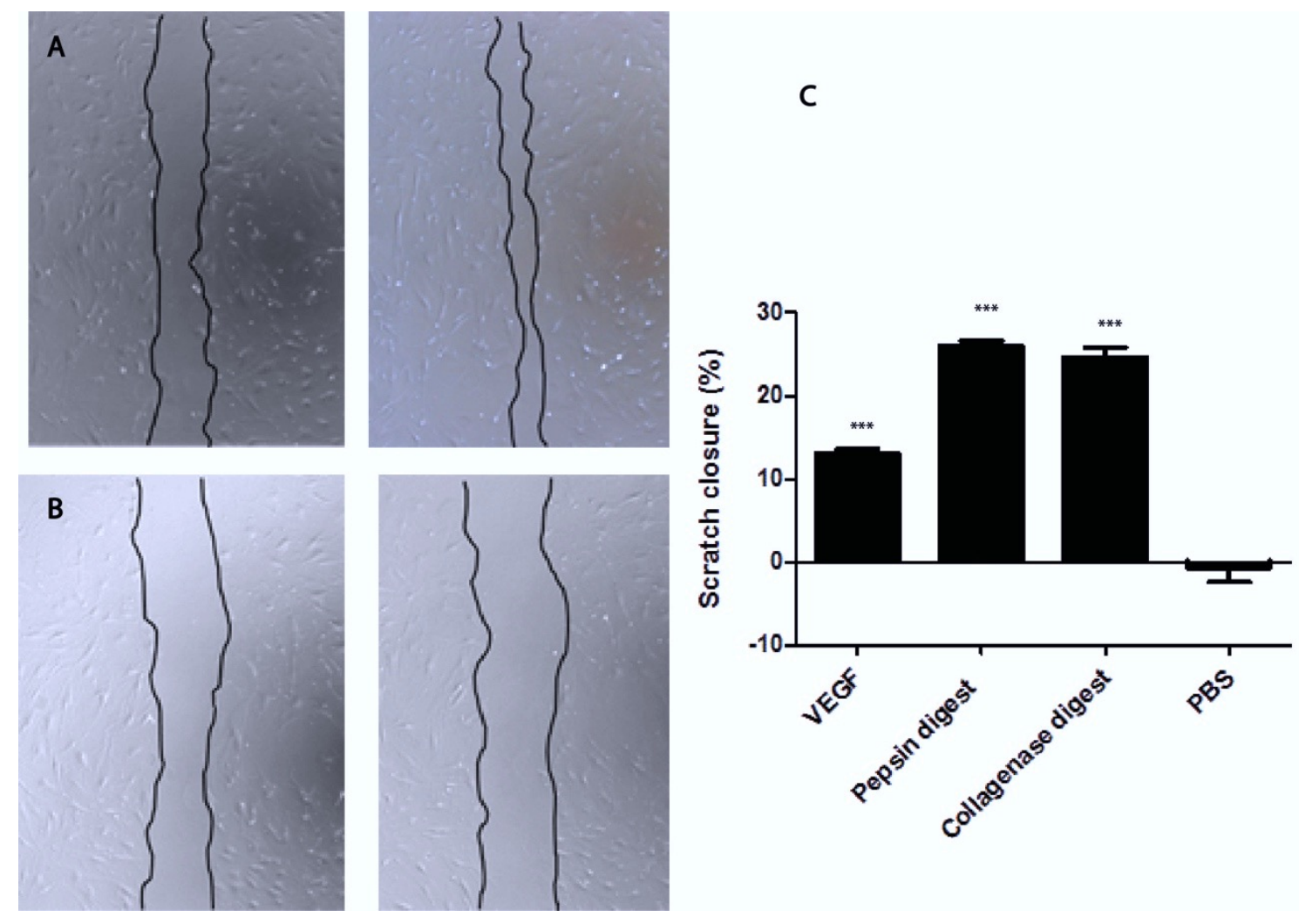

\section{Figure 3-7 MSC scratch assays}


MSC monolayers were scratched and then incubated for 12 hours with dECM digests at $100 \mu \mathrm{g} / \mathrm{mL}$ using pepsin and collagenase. Photomicrographs of the scratched areas are shown in A) VEGF control 0 and 12 hours and B) media control at 0 and 12 hours. Scale bar (white) is $50 \mu \mathrm{m}$. C) Migration was expressed and the change in scratch area for VEGF and pepsin and collagenase digested AECM proteins, and a PBS control. Error bars indicate SE in triplicate wells. Samples with significant change in scratch closure in relation to PBS control (***) were calculated by one-way ANOVA $(\mathrm{p}<0.001)$.

\section{Chemotaxis Assays}

A chemotaxis assay was used to support the scratch assays for pepsin digested samples (Appendix 5). For this method, a concentration gradient of the extract is established using a central plug embedded in agarose above the cell monolayer. The migrated cells in the centre of the well, where the extract concentration is highest, are counted. As shown in Figure 3-8, a total protein amount of $25 \mathrm{ng}$ of the pepsin digested extract was able to illicit a chemotactic response in MSCs and HUVECs, but not FBs.

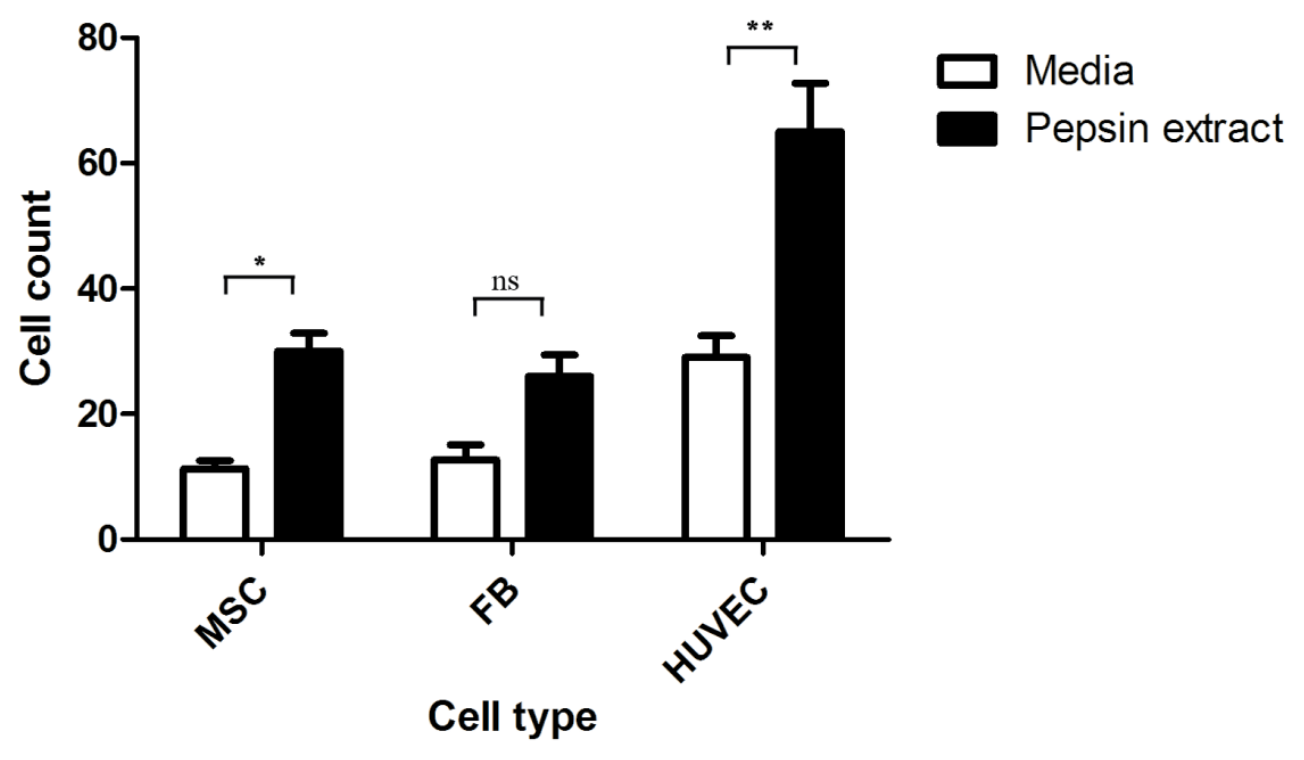

Figure 3-8 Chemotaxis assays with pepsin digested OFM

The chemotactic activity of pepsin digested OFM extracts was measured with an 
agarose plug migration assay. Cell counts are the number of cells that migrated to the centre of the well, where the concentration of the extract was highest. A total protein concentration of $25 \mathrm{ng}$ in the central well induced a chemotactic response in MSCs and HUVECs, but not FBs. Cell counts are an average from six wells, and error bars represent SE between counts. Statistical analysis was calculated using one-way ANOVA between treated (pepsin extract) and non-treated (media) for each cell type. A significant difference is seen for MSCs $\left({ }^{*} \mathrm{p}<0.05\right)$ and HUVECs $\left({ }^{* *} \mathrm{p}<0.001\right)$ but not FB $(p>0.05)$.

\section{Summary of migration activity}

The scratch assay is commonly used as an angiogenesis assay with HUVECs. FBs and MSCs do not generate as dense a monolayer as HUVECs, and may not be as suitable for this assay. In addition, the scratch assay does not model the chemotactic gradient established in the recruitment of many wound healing cells. In order to establish that the digested OFM extracts were chemotactic for MSCs, HUVECs and FB an agarose plug chemotaxis method was used. As shown in Table 3-3, the pepsin digested OFM extract showed significant chemotactic activity towards MSCs and HUVECs $(\mathrm{P}<0.05)$ using both assays, but this was not significant against the FB line.

\begin{tabular}{|c|c|c|c|}
\hline & FB & MSC & HUVEC \\
\hline PEPSIN DIGEST & \multirow{4}{*}{$\begin{array}{l}\text { NOCHANGE } \\
\text { (DATA NOT } \\
\text { SHOWN) }\end{array}$} & SIGNIFICANT & SIGNIFICANT \\
\hline \multirow{3}{*}{ (SCRATCH ASSAY) } & & INCREASE IN & INCREASE IN \\
\hline & & MOTILITY & MOTILITY \\
\hline & & (FIG 3-7) & (FIG 3-6) \\
\hline COLLAGENASE DIGEST & No CHANGE & SIGNIFICANT & SIGNIFICANT \\
\hline \multirow{2}{*}{ (SCRATCH ASSAY) } & \multirow{2}{*}{$\begin{array}{l}\text { (DATA NOT } \\
\text { SHOWN) }\end{array}$} & INCREASE IN & INCREASE IN \\
\hline & & (FIG 3-7) & (Fig 3-6) \\
\hline \multirow{4}{*}{$\begin{array}{l}\text { PEPSIN DIGEST } \\
\text { (CHEMOTAXIS ASSAY) }\end{array}$} & No & SIGNIFICANT & SIGNIFICANT \\
\hline & SIGNIFICANT & INCREASE IN & INCREASE IN \\
\hline & CHANGE & MOTILITY & MOTILITY \\
\hline & (FIG 3-8) & (FIG 3-8) & (FIG 3-8) \\
\hline
\end{tabular}

Table 3-2 Summary of migration activity

82 Dynamic reciprocity between wound healing cells and the extracellular matrix 


\subsubsection{Histology of dECMs from other tissue}

Tissue harvested from different organs was decellularised using a process similar to that used for the manufacture of OFM. Cross sections of different dECM tissues were fixed and stained to demonstrate that the tissue had been completely decellularised. Figure 3-9 shows the haematoxylin and eosin stained sections of different dECM tissues.

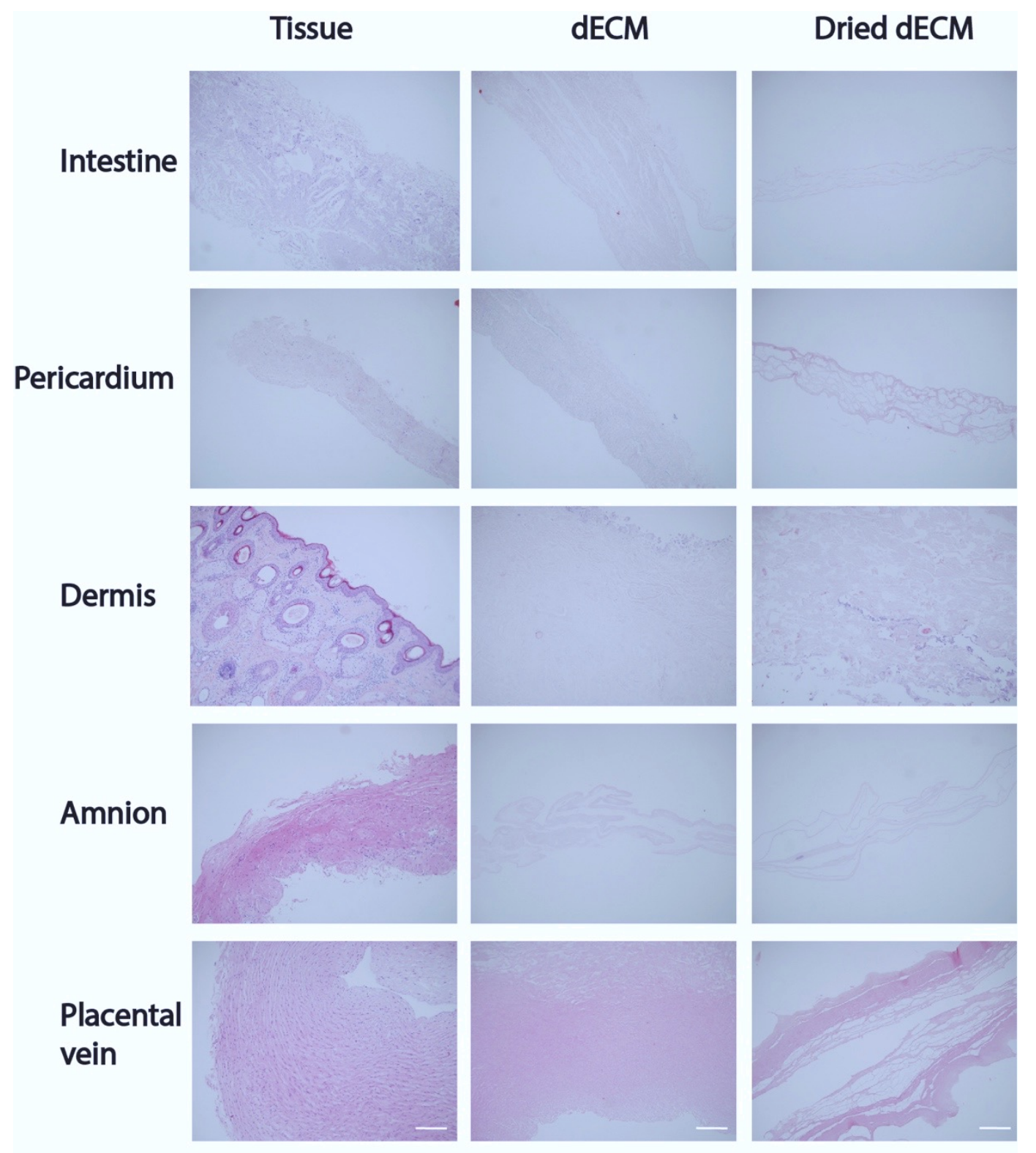

Figure 3-9 Decellularisation of different ovine tissue

Images in columns show unprocessed tissue (left), decellularised tissue (middle) and 
freeze dried decellularised tissue (right). Ovine tissue processed from different organs is shown; intestine, pericardium, dermis, amnion, and placental artery. All images were taken at 10x, scale bar (white) shows $200 \mu \mathrm{m}$ (in the last row).

In the cross sections of dECMs from other sources, distinct nuclei can be seen in the raw tissue samples on the left, while decellularised material in the centre is clear of cell bodies. In the right column the dried $\mathrm{AECM}$ has a more condensed collagen structure compared with the raw tissue and decellularised tissue.

Each tissue has distinct characteristics, depending on its origin. Intestinal tissue, which appears very similar to rumen tissue (not shown), has a submucosal ECM structure, with a dense collagen layer. Pericardium tissue has a thick muscle ECM structure with densely connected collagen fibres. Dermal tissue, with many appendages and large vessels is easily distinguished from other tissue. Placental vein tissue is highly cellular, and along with amnion tissue is very darkly stained by eosin (pink).

\subsubsection{Comparison of bioactivity in different ECM tissue}

\section{Pepsin digestion of different ovine dECM tissue}

The yield of dECMs from other tissue was variable, due to the amount of starting material available. Consequently, dECMs were digested with pepsin using a different starting mass for certain tissue, ranging from 0.5 to $1 \mathrm{~g}$. this was reflected in the protein concentration of the digested material. In Table 3-2, the starting mass of each tissue and the concentration of the pepsin digest is shown. Placental vein and artery tissue were combined. Unfortunately, this dECM led to a small yield and could not be tested for all assays.

\begin{tabular}{|c|c|c|c|}
\hline TISSUE & $\begin{array}{l}\text { MASS TO } \\
\text { VOLUME RATIO } \\
\text { IN PEPSIN } \\
\text { DIGESTION }\end{array}$ & $\begin{array}{l}\text { CONCENTRATION } \\
\text { (MG/ML) }\end{array}$ & YIELD (MG) \\
\hline RUMEN & $1 \mathrm{G} \mathrm{IN} 50 \mathrm{ML}$ & 7.4 & 375 \\
\hline INTESTINE & $1 \mathrm{G} \mathrm{IN} 50 \mathrm{ML}$ & 6.3 & 315 \\
\hline DERMIS & $1 \mathrm{G} \mathrm{IN} 50 \mathrm{ML}$ & 5.5 & 275 \\
\hline
\end{tabular}

84 Dynamic reciprocity between wound healing cells and the extracellular matrix 


\begin{tabular}{l|lcc} 
PERICARDIUM & $0.5 \mathrm{G} \mathrm{IN} 25 \mathrm{M} \mathrm{L}$ & 6.0 & 150 \\
AMNION & $1 \mathrm{G} \mathrm{IN} 50 \mathrm{ML}$ & 5.7 & 285 \\
CHORION & $1 \mathrm{G} \mathrm{IN} 50 \mathrm{ML}$ & 5.9 & 250 \\
$\begin{array}{l}\text { PLACENTAL VEIN } \\
\text { \& ARTERY }\end{array}$ & $0.6 \mathrm{G} \mathrm{IN} 30 \mathrm{ML}$ & 6.6 & 198
\end{tabular}

Table 3-3 Comparative yield of pepsin digested dECMs

\section{Proliferation}

The presence of bioactive molecules in dECMs from different source tissues was compared using an MTT assay to test a change in the proliferation of MSCs after a 48-hour incubation period. Solutions of pepsin-digested ECMs were prepared from amnion, chorionic, placental, dermis, pericardium, intestine and rumen tissue. All samples were normalised to a protein concentration of $100 \mu \mathrm{g} / \mathrm{mL}$. Figure 3-10 compares the change in absorbance by MTT after incubation with pepsin-digested extracts from different source tissue. This is expressed as the absorbance at $550 \mathrm{~nm}\left(\mathrm{~A}_{550}\right)$ of each culture, relative to a control with no cells (media).

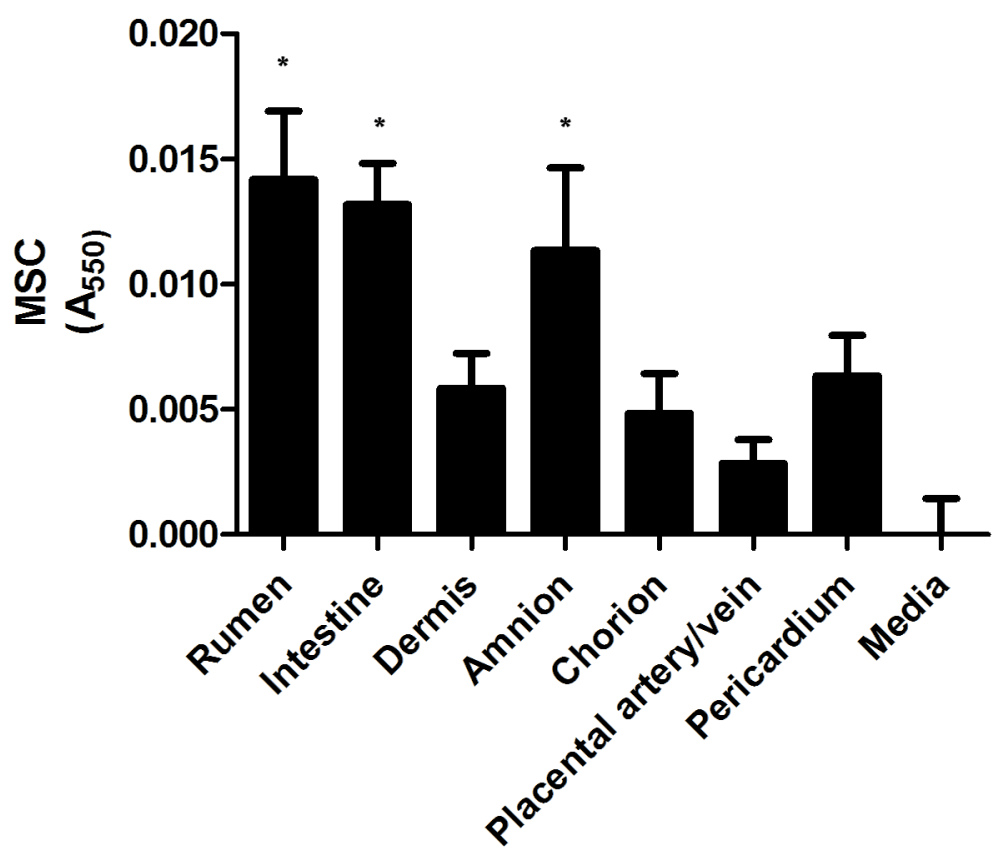


Figure 3-10 MTT assay with digested dECMs: MSCs

MSC monolayers were subject to an MTT assay after a 48-hour incubation with pepsin digested extracts of dECMs from different ovine tissue. Extracts from amnion, rumen, and intestine tissue led to a significant increase in absorbance relative to media controls. Absorbance values are the average from six wells, significant treatments compared with media control (*) calculated by one-way ANOVA (p<0.001).

\section{Migration}

Pepsin digested extracts of different tissues were tested for MSC migration using the scratch and chemotaxis assays. Figure 3-11 shows the migratory effects of pepsin digested tissue from dECMs on MSCs. Using both scratch and chemotaxis assays, the samples varied in their effect on MSC motility. All pepsin digested samples directed an increase in cell migration using the chemotaxis assay, the sample derived from amnion dECM demonstrating the greatest effect on MSC chemotaxis. In the scratch assay, a similar trend is seen, however rumen, amnion, vein and pericardium were the only samples which were significant by the standards set in this analysis $(\mathrm{p}<0.05)$. Unfortunately, the placental vein and artery samples could not be tested in the chemotaxis assay, due to a low yield of this dECM. 

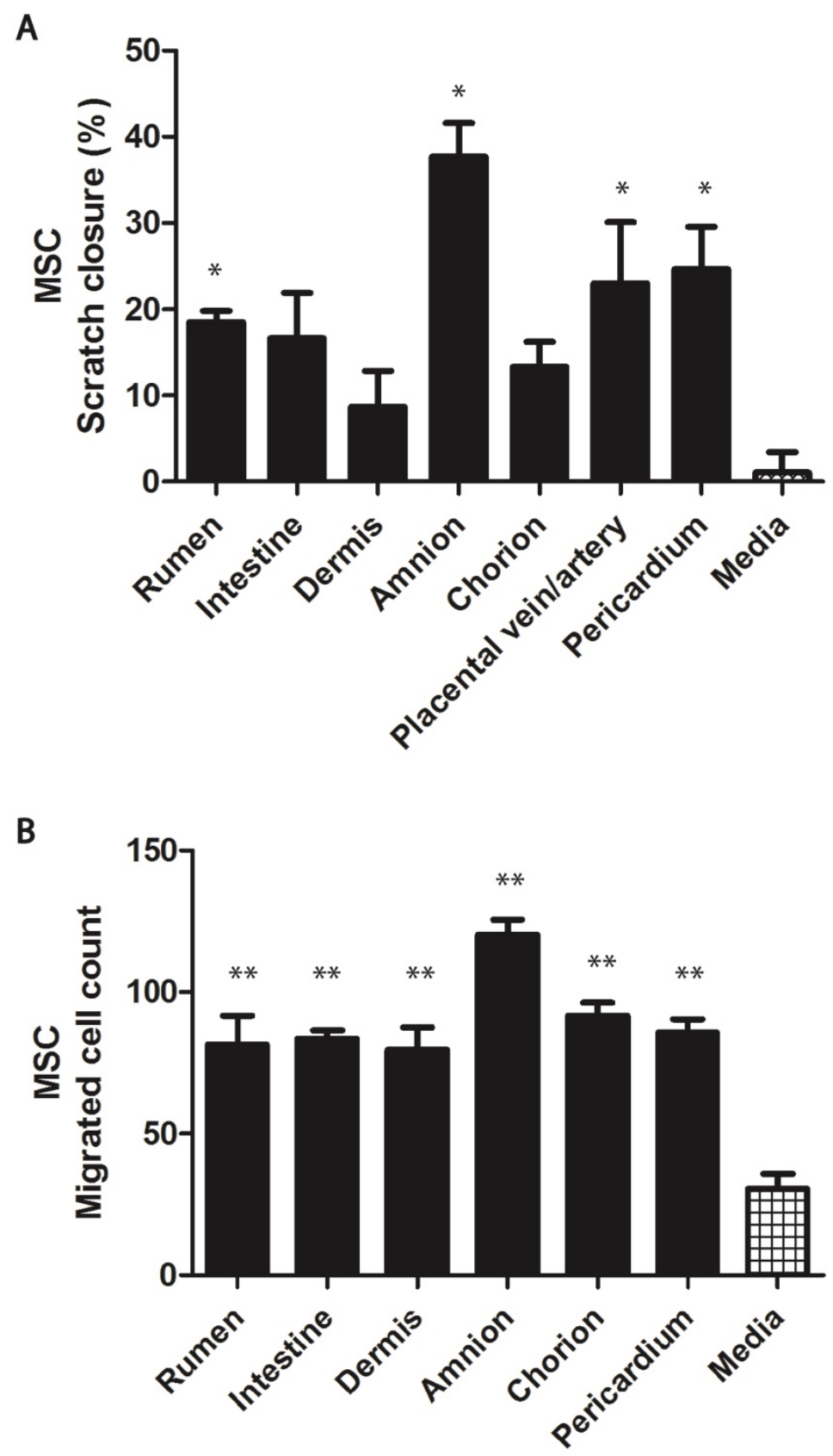

Figure 3-11 MSC migration with digested dECMs

MSC monolayers were subject to migration assay after an incubation with pepsin digested dECMs from different tissues. MSC scratch assay (A) showed a significant increase in scratch closure using rumen, amnion, placental vein and pericardium dECMs. Significant values for the scratch test (*) were calculated by one-way ANOVA compared with media control from 3 wells * $(p<0.05)$. MSC migration using the scratch 
assay (B) showed a significant increase in MSC chemotaxis in all dECMs. Significant values for migrated cell count (**) were calculated by one-way ANOVA compared with media control from six wells ${ }^{* *}(\mathrm{p}<0.001)$.

Pepsin digested extracts of different tissues were tested for HUVEC migration using the scratch and chemotaxis assays. Figure 3-12 shows the migratory effects of pepsin digested tissue from dECMs on HUVECs. Using both assays, the samples differed in their effect on HUVEC motility. All pepsin-digested samples gave a significant increase in the scratch assay relative to the media control; foetal tissue (amnion and chorion) and pericardium tissue leading to the greatest relative scratch closure. This pattern was also observed in the chemotaxis assay, with some exceptions. The dermal and amnion dECMs did not appear to have a great impact on motility using this method. 
A
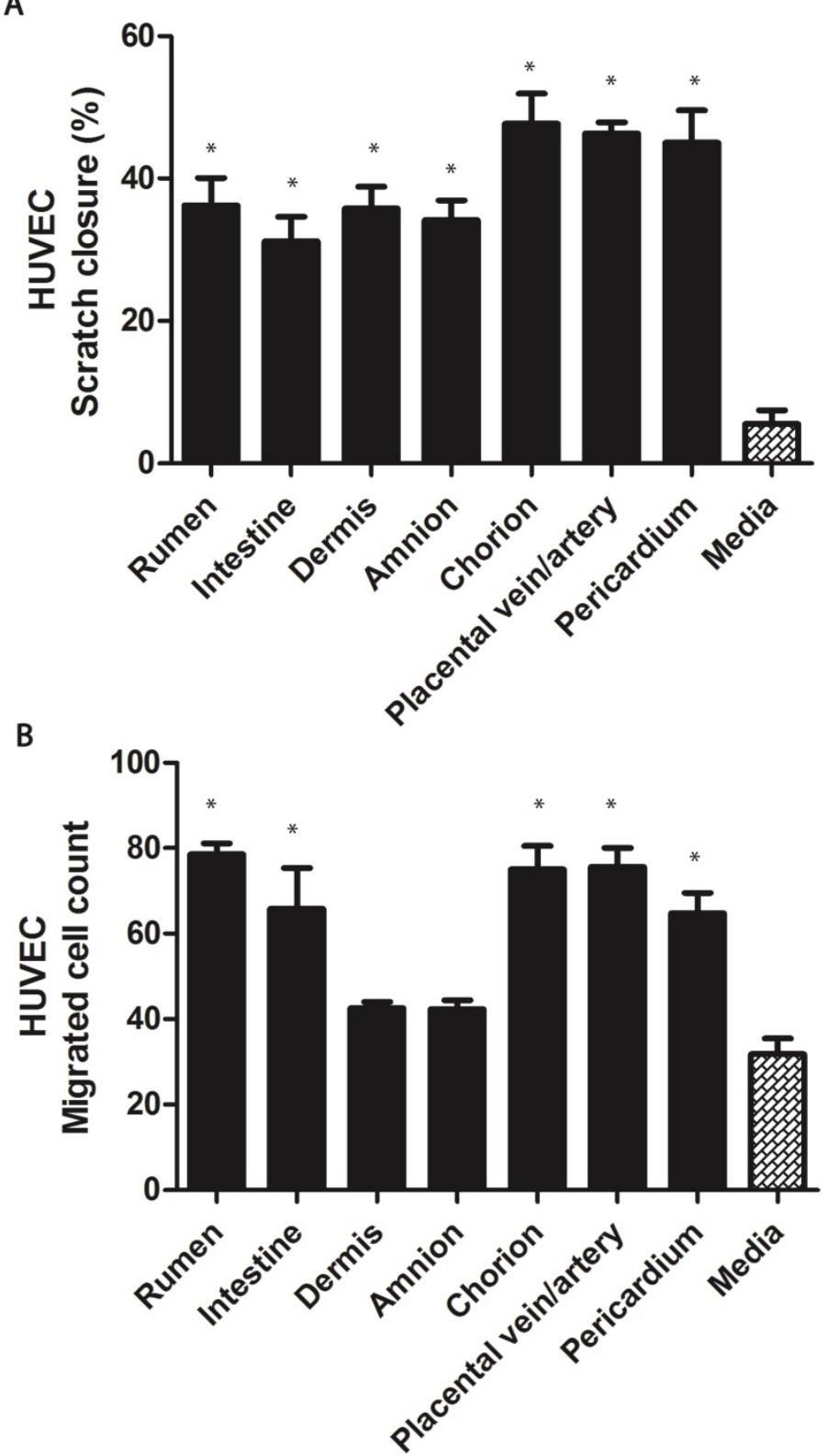

HUVEC

Figure 3-12 HUVEC migration with digested dECMs

HUVECs were subject to migration assays with extracts of digested AECM from different ovine source tissue. HUVEC scratch assay (A) showed an increase in scratch closure using all samples. Significant values for the scratch test were calculated by one-way ANOVA compared with media control (* $\mathrm{p}<0.001, \mathrm{n}=3)$. HUVEC migration using the chemotaxis assay (B) showed an increase in chemotaxis in rumen, intestine, 
chorion, placental vein and pericardium dECMs. Significant values for migrated cell count were calculated by one-way ANOVA compared with media control ( $\mathrm{p} p<0.001$, $\mathrm{n}=6)$.

\subsection{Discussion}

A number of the properties of a $\mathrm{dECM}$ material may account for their regenerative capacity in vivo. These biomaterials retain the structure of living ECM proteins and accessory molecules are preserved. In addition, the material is an aid to the reconstruction process as a physical scaffold that cells can attach to and infiltrate. If the role of the dECM in wound healing is above that of a simple scaffold for wound healing cells, one would assume that the dECMs contain intrinsic bioactive properties that affect the behaviour of wound healing cells.

\subsubsection{Attachment, proliferation and infiltration of OFM by cells}

\section{Attachment}

Cells must be able to attach to a dECM biomaterial before they can begin to incorporate it into the healing wound. Section 3.4.1 shows that the greatest attachment occurred on OFM discs pre-conditioned with serum. The significant change in cell attachment in relation to FBS in the conditioning medium is important when considering experiments downstream, such as proteolytic digestion and cellular infiltration of the material. Notably, these cells can attach to normal plastic surfaces in the absence of FBS, indicating that the material is not a good surface for cell attachment without the pre-conditioning step.

The time for attachment in this experiment is short, just one hour. It is therefore unlikely that the results shown are due to an increase in cell number by proliferation. The FBS may interact with the surface of the material making it more adhesive. As FBS is mostly composed of albumin type proteins, these may coat the surface of the OFM in the same 
way that denatured collagen products such as rat tail collagen and gelatine can be used to coat tissue culture plates.

Denatured collagen products, such as rat tail collagen, are very effective substrates for cell attachment. The surface of the AECM material is more reminiscent of normal tissue ECM, with intact collagen fibrils, elastin and other ECM proteins. Glue-like denatured collagen or gelatine may be more akin to the provisional matrix in the early stages of wound healing, which is made of fibrin and a superb substrate for cell attachment. OFM is composed mostly collagen I, so in theory cells should attach readily to it. The matrix in living tissue is normally in a gel-like state; collagen appears swollen and hydrated with proteoglycans and accessory GAG proteins. The loss of this gel-state during processing may alter the way in which cells attach to the material.

The MTT assay is a simple way to quantify a cell population, with the admonition that it is a measure of metabolic activity and not cell number. It is therefore likely that the residual FBS in this experiment contributed to the increase in metabolic activity observed. However, images of the discs qualitatively show that more cells are attached to discs preconditioned with FBS and DMEM with $10 \%$ FBS (Figure 3-1), therefore it is assumed that the change in absorbance is the result of a higher number of cells populating the device and not purely an increase in the metabolic activity of these cells.

Biomaterials such as OFM integrate into the host in vivo without a detrimental inflammation response or cell death ${ }^{1}$. However, this study shows that the level of cell attachment is amplified by pre-conditioning the material in FBS. The in vivo studies show that monocytes, FBs and endothelial cells can all attach to and survive within the AECM in vivo. Is the conditioning with FBS in vitro allowing the material to act as it does in the host, saturated by highly concentrated protein solutions from blood and interstitial fluid? The presence of proteases, serum and other proteins in a living wound may act to condition the $\mathrm{dECM}$ in a similar way. At this point it may be relevant to ask, is a greater level of cell attachment desirable for wound healing products? Some devices show greater healing capacity when they have been coated or conditioned in platelet rich plasma before 
implantation ${ }^{193}$. It is possible, that conditioning this kind of material in a patient's own serum would lead to greater cell attachment after implantation of the device.

From this result, it is concluded that cell attachment in vitro is affected by the presence of FBS, either as a residual component of the experiment or used to condition the dECM discs before cell seeding. The consequences of using FBS may have considerable downstream effects on culture models.

\section{Proliferation and infiltration}

Cells grown on dECMs were sectioned, stained and imaged microscopically. In theory, this would demonstrate whether a cell line is capable of proliferating and infiltrating the material. This method of counting cells per section is indicative of a change in the cell number, not a change in metabolic activity as is the case in the MTT assay. MSCs were able to proliferate on the material, as shown by the increase in the number of cells counted on each section over time in Figure 3-4. This experiment demonstrated that OFM acts as a scaffold for MSC attachment and allow for cell proliferation, after an initial drop in number. This finding was also shown by Giavaresi et al. in an experiment where both differentiated and non differentiated MSCs proliferated on a dECM derived from dermal tissue $^{194}$.

Figure 3-3 shows that cell growth on OFM is confined to the seeded surface. Modifications to the number of seeded cells and the length of incubation did not alter this. This effect was seen in FBs, HUVECs, MSCs and EOMAs. The lack of infiltration into the dECM in vitro is a significant finding.

\subsubsection{The use of OFM as a three dimensional cell culture substrate}

Studies have shown that the scaffold is a positive mediator of wound healing; therefore, the ability of the scaffold to host wound healing cell attachment and proliferation in vitro was expected. Despite in vivo studies that have shown the infiltration of FBs and monocytes and even the organisation of endothelial cells into vessels within the matrix, it 
seems that the material is ill adapted for in vitro culture or more complex model systems in its current state.

\section{Proteolytic activity and the absence of other cell types}

Many cells express proteases as they infiltrate the ECM of living tissue. Leukocytes, particularly neutrophils and macrophages, are required to break down the injured tissue and debris during wound healing. Proteases for breaking down OFM and generating cues for FB organisation and infiltration are absent from this model. While MSCs and FB generate proteases themselves, the OFM in this form might be too difficult for such a small number of cells to breakdown. Growing a "co-culture" of different cell types such as macrophages and FBs could lead to improved infiltration of OFM.

\section{Collagen stiffness}

Another consideration for three dimensional culture methods is the collagen stiffness of a culture. FBs have a mechanical function and are responsible for maintaining tension homeostasis by the contraction of collagen fibres in connective tissue. The models often used in three dimensional cell culture incorporate collagen solutions of less than $2 \%$, in order to demonstrate the behaviour of small number of FB cells ${ }^{104}$. Native collagen can be hundreds if not thousands of times stiffer than these free floating collagen gels. The OFM scaffold has a strength and stiffness closer to that of living tissue and may represent a more relevant model, but the the ratio of cells to collagen fibres is not representative.

Dutta et al. suggested that hydrogels make excellent three dimensional models for cell culture due to their ability to retain water ${ }^{103}$. This gives the hydrogel properties which make it more amenable to cell motility such as a high porosity that allows a free flow of oxygen and nutrients. In addition, cells in a hydrogel are in a more "stretched" position, compared with cells in a monolayer; this could lead to greater contact between integrins and ECM proteins and thus promote motility. 
Matrix stiffness will alter the level of cell migration in a hydrogel model and the compliance of a collagen gel has shown to influence the type of migration observed ${ }^{195}$. Rigid gels encourage a rho-dependent migration pattern and loosely compacted gels allow amoeboid migration. As discussed in the introduction, the provisional matrix in normal wound healing is more pliable for migrating FBs.

Some ECM-based materials, such as fibrin and Matrigel ${ }^{\circledR}$, allow for cell infiltration and form three dimensional cell cultures in vitro. Unlike OFM, Matrigel ${ }^{\circledR}$ and fibrin plugs are gels at ambient temperature. In addition, the recombinantly expressed proteins of Matrigel ${ }^{\circledR}$ are further solubilised with proteolytic enzymes giving a viscous liquid at colder temperatures. It is likely that the solubilisation process can account for the ease of cellular infiltration, because the collagen fibres are significantly softened in this process making them more susceptible to cell directed proteolysis preceding cell invasion.

Matrigel ${ }^{\circledR}$, and other acellular matrices are fully hydrated and accompanied by accessory GAGs, growth factors, enzymes, and high levels of laminin and collagen $\mathrm{IV}^{103}$. The high laminin content of Matrigel ${ }^{\circledR}$ causes the polymerisation of the matrix to a gel-like substance at temperatures higher than $4^{\circ} \mathrm{C}$. These physical attributes make the material a successful in vitro cell scaffold.

The ECM of Matrigel ${ }^{\circledR}$ is generated from cancer cells, another reason the matrix is more receptive to cellular migration, infiltration and tube formation. Matrigel ${ }^{\circledR}$, expressed by a murine sarcoma cell line, may give an exaggerated model of infiltration since tumour ECM promotes angiogenesis more than normal ECM tissue, which acts to control the process. Genovese et al. demonstrated that cancer cells and peripheral mononuclear blood cells could infiltrate the $\mathrm{dECM}$ derived from colorectal carcinoma tissue more readily than a dECM derived from the "perilesional" area ( $1 \mathrm{~cm}$ away), and minimal infiltration was seen in a dECM from healthy tissue ${ }^{196}$. The ECM in normal tissue acts as a guard against invading tumour cells, which may be why infiltration is suppressed in $\mathrm{dECM}$ from normal tissue.

Serum compromises the infiltration of FBs in vitro 
The presence of serum in the culture will inhibit many of the proteases required for cell infiltration. This makes the initial finding that pre-conditioning of OFM is required before cell seeding highly relevant.

Studies using semi-solid collagen gel matrices described by Rhee et al. ${ }^{28}$, have indicated that the FB phenotype is pro-migratory/infiltrative or pro-contractile, depending on the presence of PDGF or serum proteins respectively. PDGF induced infiltration of FBs into the collagen plugs, while serum prompted a phenotypic change in the cells to induce shrinkage and reversible contraction of free floating gels. It is likely that the pre-treatment of the scaffold with serum and the use of serum during the proliferation experiments inhibited the FB infiltration of OFM. If the material is to be investigated further as a three dimensional cell culture model, the cells would have to be able to attach to it and grown in reduced or no-serum culture media, with added stimulation from PDGF if needed.

\subsubsection{Bioactivity of soluble and pepsin digested components of OFM}

Breaking down $\mathrm{dECM}$ proteins may also be the key to finding out how dECMs improve the wound healing outcome in vivo. The aim of these assays was to establish the bioactive potential of OFM in assays that are relevant to wound healing. Learning how these materials alter wound healing cell behaviour could lead to new insights into the mechanisms of dECM-mediated wound healing.

\section{An increase in proliferation is observed when OFM is processed by proteolysis}

Bioactivity assays were employed to test the effect dECM proteins may have on wound healing cells. The dECM extracts dissolved in PBS, cell culture media and bicarbonate show no significant activity in the proliferation assays (Table 3-1). The preparation of the dECMs involves a number of washes with salt and detergent. Soluble bioactive components of the ECM will wash off during these steps, revealing hidden molecules within the material architecture. For this reason, the material was prepared for bioassays using an enzyme to break the material down. 
A significant increase in MSC cell number is observed when the monolayer was incubated in pepsin digested OFM. All three samples shown in Figure 3-5 have been normalised to the same protein concentration, but the sample that has been digested the longest (sample $\mathrm{C}, 48$ hours) leads to the greatest increase in metabolic activity. This indicates that longer digestion of the material releases more bioactive ECM molecules. This result is confirmed by a proliferation assay using cell counts after incubation with sample $\mathrm{C}$. This shows the number of cells was significantly higher after an incubation with this digest and not simply due to an increase in metabolic activity shown by MTT.

A number of cell types are activated to a highly proliferative state during wound healing, the presence of mitogens in the OFM digests would make the material a promoter of wound healing. Because cells and proteases in living tissue digest OFM, this way of extracting bioactives by enzymatic digestion may be a good way to find promoters of wound healing in such materials.

\section{An increase in motility is observed when OFM is processed by proteolysis}

Proteolytic digests of OFM were also tested for their ability to induce cell motility using scratch and chemotactic assays with HUVECs, MSCs and FBs. As summarised in Table 32, pepsin and collagenase digested OFM led to an increase in cell motility for HUVECs and MSCs but not FBs. In Figure 3-8, it appears that FBs did respond to a chemotaxis assay but the result was not significant in relation to the media control. It is possible that because FBs are highly migratory without stimulation, the cells in this assay did not respond to this concentration of the pepsin digested extract.

A number of studies have shown that ECMs and their breakdown products can recruit cells in vitro and in vivo, as reviewed by Swinehart et al. ${ }^{1}$. Recruiting cells necessary for the constructive remodelling of injured tissues could be one of the ways that dECM biomaterials are able to promote wound healing.

96 Dynamic reciprocity between wound healing cells and the extracellular matrix 


\subsubsection{Bioactivity of dECMs derived from different source tissue}

ECMs possess different attributes depending on whether they are required for strength, elasticity or durability. Different tissues also have a different capacity to regenerate and heal after injury. In order to test whether these differences correspond to dECMs with different bioactivity, tissues from different sources were decellularised and digested with pepsin for proliferation and migration bioactivity assays.

Section 3.4.5 described the comparison of dECMs from different ovine tissues. In Figure 39, sections of these tissues before and after decellularisation show that nuclei are mostly removed from all tissue types. This indicates that the method used across all tissue has been successful in decellularisation. Interestingly, the drying step, rather than the decellularisation step, significantly alters the ECM structure.

The methods used for decellularisation were optimised for OFM, and then used on all tissue with dermal tissue requiring an additional salt step to remove keratin. Ideally, the decellularisation of different tissue would have been optimised for each, however this would have required a much larger sample of tissue, some of which are difficult to source (such as amnion and chorion). The salt step introduced for the dermal tissue may have depleted the dermal dECM of bioactive components compared with the others.

Different tissues also rendered a variation in yield. As shown in Table 3-3, placental vein/artery and pericardium tissue yielded significantly less ECM than other tissue.

Another consideration is the method of dECM break down. Pepsin digestion of samples was carried out because of the effect seen on cells cultures in OFM pepsin extracts (sections 3.4.3 and 3.4.4). Other tissue may require shorter or longer digestion times, or require a different protease to release bioactives.

\section{Proliferation activity}

The bioactivity of extracts from rumen (OFM), intestine, pericardium and amnion dECMs after pepsin digestion was significantly higher than media controls, in relation to MSC 
proliferation (Figure 3-10). The dermal, chorion and placental vein tissue did not produce pepsin digested extracts that induced MSC proliferation in this assay.

DECM derived from intestinal and rumen tissues, which are constantly self-renewing, may retain wound healing promoting molecules that are released by proteolytic digestion. The proximity to harsh conditions such as a low $\mathrm{pH}$ in the intestinal tract may make this ECM more robust in relation to the loss of bioactives during the decellularisation process. As mentioned above, the decellularisation processes used were optimised for these tissues. It is possible that these tissues interact with pepsin in a different way since they are located near high concentrations of the enzyme in the stomach. The enzymes may be overdigesting the other $\mathrm{dECMs}$ by comparison.

The amnion tissue gives a highly bioactive pepsin-digest in relation to MSC proliferation. The evidence that foetal tissue is more regenerative than adult tissue is supported by many studies $^{197}$. Curiously, this effect was not observed in the chorion tissue digest. There are differences in ECM composition of these two membranes. Amnion tissue is derived from the membrane surrounding the foetus and consists of collagens, laminin, fibronectin, proteoglycans and nidogen. Chorion tissue, the maternal membrane has only a reticular layer, basement membrane and trophoblast layer. Foetal tissue is a fascinating avenue to explore, it is possible that bioactives discovered from these dECMs could be synthesised for use in wound healing and tissue generation. Liver tissue is also highly regenerative. It would be interesting to measure the availability of wound healing molecules from a dECM made from this tissue. However, the process of decellularising whole organs is more complicated than that of flat sheets of dermis or rumen.

\section{Migration activity}

MSC migration was also tested using the scratch assay and chemotactic assay (Figure 311). Amnion dECM extracts led to the greatest MSC motility in both assays. This indicates that this $\mathrm{AECM}$ is highly active in both MSC proliferation and migration. Interestingly, the chemotaxis assay shows that all extracts are chemotactic for MSCs in the chemotaxis 
assay. Using the scratch assay, this change in cell motility is not as clearly defined and dermal and chorion tissue do not have a significant effect on motility in the scratch assay. Using HUVECs the opposite effect is seen (Figure 3-12). Whilst all extracts led to HUVEC motility, the difference between extracts and controls is greatest in the scratch closure assay. Interestingly, chorion, placental vein and artery and pericardium dECMs show the highest change in cell motility in the HUVEC scratch assay.

This suggested that the breakdown of almost any ECM would generate a chemotactic gradient, given the nature of ECM breakdown in wound healing. It is possible that this effect is not as clear in the scratch assay because this model is not appropriate for MSCs; these cells do not form confluent monolayers and do not migrate as endothelial cells do in a sheet with leading edge migratory cells and a proliferating sheet of cells pushing them forward.

\subsection{Conclusion}

These experiments show that wound healing cells will attach to and proliferate on, but not infiltrate into OFM in vitro. This is in contrast to studies that show cells such as FB and endothelial cells can infiltrate, form microvasculature and eventually breakdown the material in vivo, during constructive remodelling. This may be due to the absence of immune cells and proteases in the in vitro model.

Pepsin-digested OFM extracts incubated with MSCs for 48 hours led to a significant change in MSC proliferation. This was not observed in FBs and HUVECs, possibly because the FBS required by these cell line in the culture media, masks the effect of the extracts. Motility assays, using pepsin and collagenase-digested OFM, show that these extracts are chemotactic for both HUVEC and MSC.

The decellularisation method used to generate OFM can be applied to other types of tissue, with minor modifications. The bioactivity of these dECMs was quantified after pepsin digestion. Amnion, pericardium and rumen tissue digests induced MSC proliferation in an MTT assay. All dECMs induced HUVEC scratch closure and all extracts were 
chemotactic towards MSCs. Amnion tissue was more chemotactic than any other extract for MSCs.

These assays clearly show that dECMs broken down by proteases release bioactive components involved in cell proliferation and migration. Interestingly, different tissues may be more or less active towards different cell types. Many of these findings could be investigated further to include the isolation of specific bioactives within extracts. Different proteases, such as MMPs could be used for AECM breakdown and tissues such as whole organs could be decellularised. Proliferation and migration assays could be carried out with other wound healing cells such as keratinocytes, pericytes and macrophages. Finally, other wound healing assays could be employed to validate these results such as in vivo MSC recruitment assays. This would show that MSCs are recruited by dECM components during constructive remodelling. 


\section{Cell-mediated release of bioactive}

\section{ECM molecules}

\subsection{Summary}

Biomaterials such as OFM are completely degraded in vivo within 1 to 6 months. In Chapter Three, it was observed that the proteases pepsin and collagenase broke down the material to give a solution of bioactive peptides that were chemotactic for HUVECs and MSCs. In this chapter, cell-mediated degradation of OFM is explored as a more physiologically relevant means of revealing bioactives that promote wound healing. Macrophages are vital in the clearing of bacterial and ECM debris during wound healing and were used to mediate the digestion of dECM. The activity of macrophage-OFM conditioned media (CM) was analysed in terms of MSC migration.

\subsection{Introduction}

The ECM is an abundant source of wound healing promoters, as outlined in the introduction. Studies have shown that $\mathrm{dECMs}$ retain a number of bioactive components and there are examples in the literature of bioactive molecules being isolated from $\mathrm{dECMs}$ by enzymes and acid hydrolysis ${ }^{182}$. During wound healing, leukocytes facilitate matrix breakdown by acid hydrolysis and the release of proteases.

The findings outlined in the previous chapter demonstrate that there is an abundance of bioactive components in biomaterials such as OFM that require enzymatic "liberation" from their solid structure. ECM breakdown with pepsin and collagenase led to a significant increase in bioactivity. This made sense in the framework of the wound healing schedule, and to a certain extent explains why wound healing cells exhibited no infiltrative capacity in vitro, despite ample evidence that cells infiltrate these materials in vivo. Pepsin 
randomly cleaves ECM proteins into smaller parts, but is generally known as a digestive system protease. Collagenase is more abundant during wound healing, but it is not the only protease present. If enzymatic breakdown of the AECM leads to a bioactive solution, then wound healing enzymes may generate bioactives are further involved in the process.

In theory, a variety of proteases may breakdown the dECM in a way that liberates bioactive moieties. In practice, with so many enzymes involved in the dynamic wound healing environment, each expressed at different levels as wound healing progresses, screening for bioactives this way could become extremely complex. There is also a chance that products cleaved by more than one enzyme may be missed.

In this chapter, a different tool is employed to generate ECM extracts for wound healing; the wound healing cells themselves. The immune response during wound healing has two principal functions: to clear infection and to clear debris in the healing wound. Rather than attempting to break down OFM with a complex mixture of proteases that are present in wound healing, this chapter investigates the cells producing these enzymes.

\subsubsection{Protease expression by cells in wound healing.}

MMPs are essential for a number of morphogenic processes, such as mammary tissue involution, and are implicated in a number of pathologies including tumour invasion, Marfan syndrome and chronic wounds ${ }^{81-83}$. The discovery of more MMPs has led to a better understanding of how the balance of proteolysis is maintained in living tissue.

In wound healing, proteases are expressed by both inflammatory cells, such as neutrophils and macrophages, and resident wound cells such as epithelial cells, FBs and vascular endothelial cells. Many of these cells express proteases to clear debris and detach themselves from matrix proteins, allowing them to migrate and proliferate. The cells with the greatest capacity to breakdown matrix proteins are the immune cells that clear space, recruit other cells and fight infection.

The expression of proteases by cells in vitro can be established in a number of ways. A simple colorimetric way to determine proteolytic activity is to pre-label a substrate with an 
azo dye such as Congo red. Congo red binds non-covalently to proteins with a high affinity for fibres, consequently it has been used to stain amyloid deposits in tissue sections and used as a dye for cellulose materials in textiles. It is a useful dye for the measurement of proteolytic activity because its absorbance can be measured spectrophotometrically.

\subsection{Aims}

In this chapter, cells rather than enzymes are used to mediate OFM breakdown. The resulting CM from cell-OFM cultures contains proteins expressed by cells, soluble proteins that come into solution from the OFM and "liberated" proteins that come into solution as a result of cell-OFM proximity. The CM from different combinations of cells cultured with OFM was tested for bioactivity using the chemotactic migration assay.

\subsection{Results}

\subsubsection{Proteolytic activity of macrophages}

\section{Congo red released by enzymatic digestion of OFM by macrophages}

In order to determine whether macrophages grown in culture with OFM were digesting the OFM, discs were pre-stained with Congo red and then seeded with macrophages. The release of Congo red was quantified using a plate reader to demonstrate a change in absorbance compared to media alone. The level of Congo red in the media after 24 hours was plotted in relation to the number of cells seeded on the material as shown in Figure 41. Congo red leached into the media from discs without cells, but an increase in the absorbance was observed relative to the number of cells seeded on the Congo red stained OFM. 


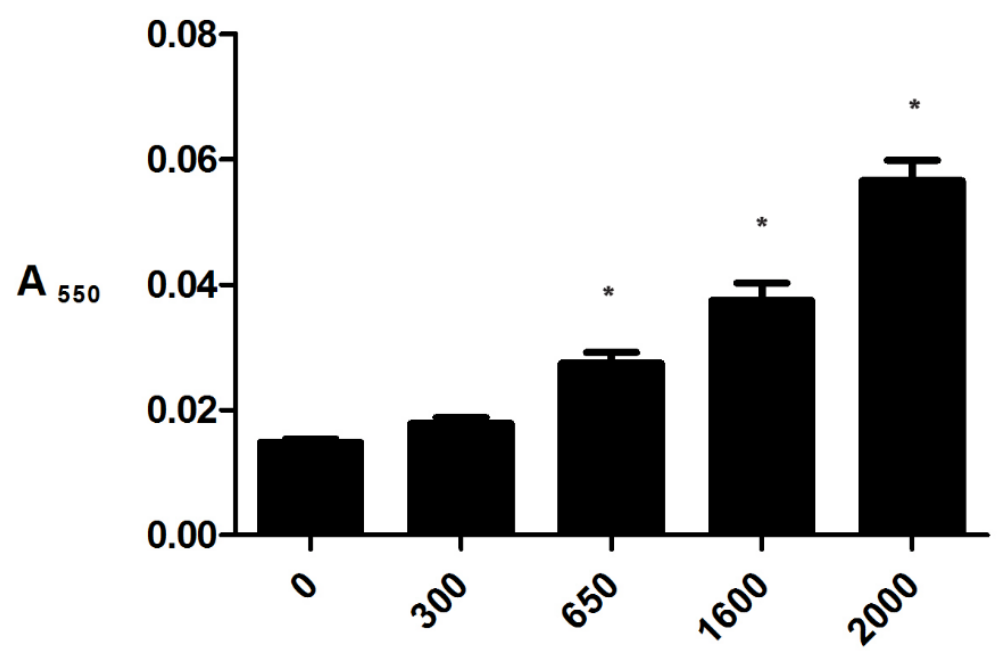

\section{Macrophage seeding density (1000 cells $/ \mathrm{mL}$ )}

Figure 4-1 Congo red release by seeded macrophages

Pre-stained OFM discs were seeded with macrophages and the level of Congo red released into culture media was measured. The release of Congo red was proportional to the number of cells seeded on the discs. A minimum of 650 thousand cells was required to generate a significant change in Congo red release compared with no cells, as calculated by one-way ANOVA of samples compared with no cells * $(p<0.001)$.

Congo red uptake by macrophages

Macrophage cells used in the Congo red release assay were gently scraped off stained OFM and non-stained OFM discs and seeded onto coverslips. These cells were imaged using an inverted microscope (Figure 4-2). The cells that had been grown on Congo redstained OFM had taken up the dye during culture. This could be due to leached Congo red attaching to the macrophages or because stained OFM proteins have been phagocytosed by macrophages. 

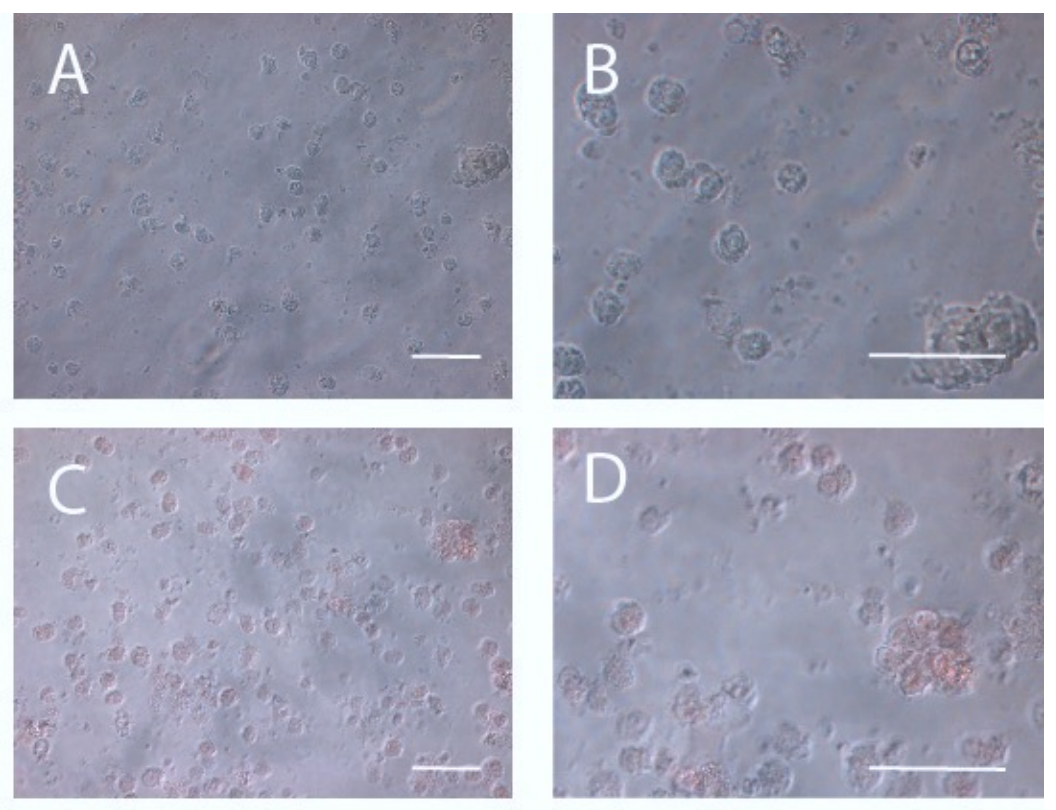

Figure 4-2 Congo red uptake by macrophages

Macrophages cultured on Congo non-stained (A and B) and red-stained OFM (C and D) were imaged after culture. Macrophages from the red-stained OFM have taken up dye and appear red compared with macrophages grown on non-stained OFM. Scale bar (white) is $50 \mu \mathrm{m}$

\subsubsection{Bioactivity of OFM cell culture CM}

In the following experiments, $\mathrm{CM}$ was collected by growing macrophage and FBs on OFM in a culture. Samples of CM were collected, filtered and testing for bioactivity using the chemotactic migration assays. Because many cell lines release chemotactic cytokines on their own, CM was collected from cells alone and from OFM alone as controls.

\section{The effect of OFM-cell CM on MSC migration}

MSC chemotaxis, using the agarose plug method, was measured using collected CM. The results are shown in Figure 4-3, along with a dose response to the chemokine PDGF. The cell count is the number of cells that have migrated to the central plug, relative to the number of migrated cells counted in wells with media alone. 
A
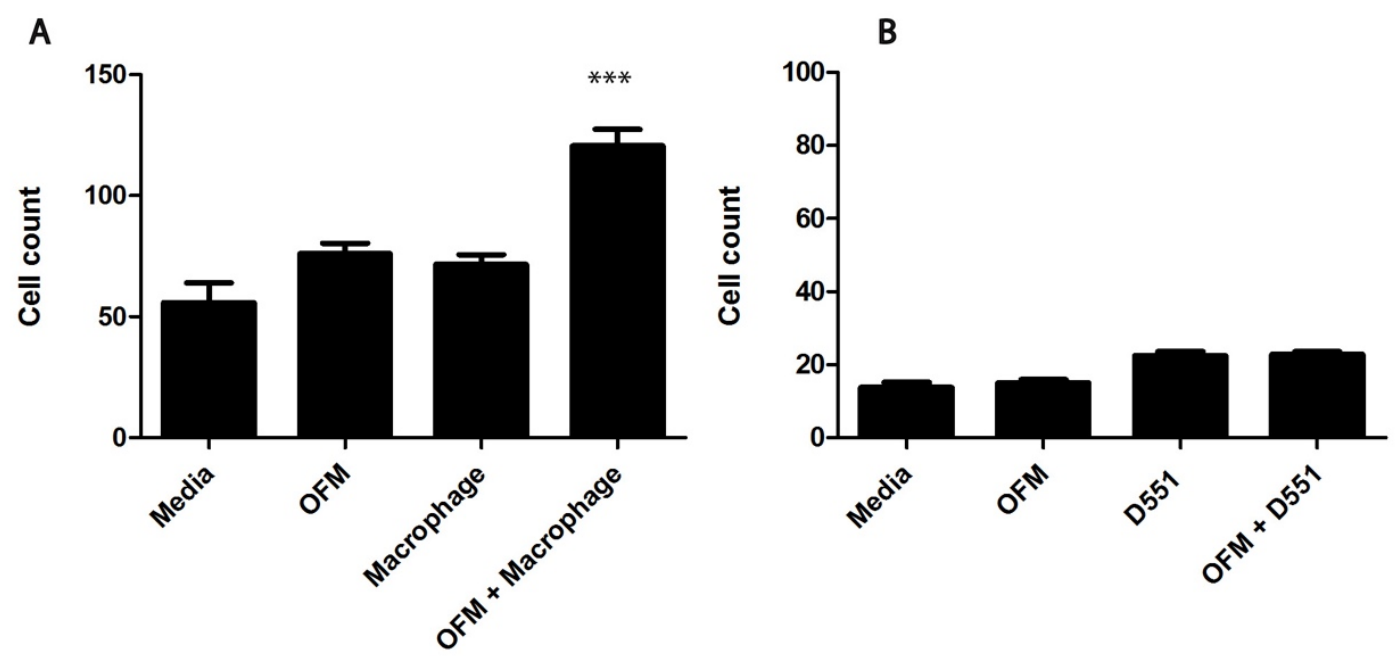

$c$

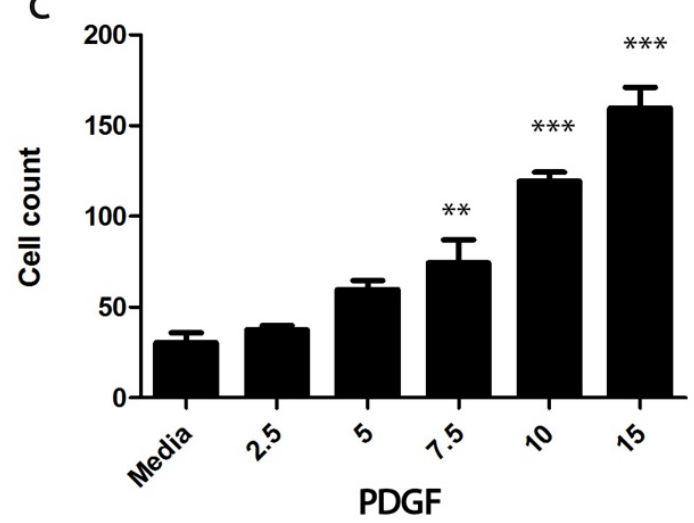

Figure 4-3 Chemotaxis of MSCs in response to CM

(A) MSC chemotaxis was tested using CM collected from OFM, macrophages and macrophages + OFM. A significant increase in chemotaxis was observed with OFM + macrophage $\mathrm{CM}$, in relation to the media control, but not the OFM and macrophage controls. This data is representative from three separate experiments. (B) MSC chemotaxis was also tested from CM collected from D551 and D551 + OFM, with a modest increase in chemotaxis towards both D551 and OFM + D551 CM. (C) MSCs responded to growth factor PDGF (shown in ng) in a dose dependent manner. Significant increase in chemotaxis was calculated by one-way ANOVA compared with media alone ( $\mathrm{n}=6$ wells, ${ }^{* *} \mathrm{p}<0.05$ and $\left.{ }^{* *} \mathrm{p}<0.001\right)$.

Figure 4-3 A shows there is a small increase in cell migration towards macrophage CM and OFM CM, however a significant increase is seen in the macrophage + OFM CM. The same experiment using CM from D551 FB cells showed no significant difference 
indicating that the OFM-FB CM is not chemotactic (Figure 4-2 B). To put this data into perspective, a dose response of MSC chemotaxis is observed using PDGF in the range of 0 $-15 \mathrm{ng} / \mathrm{mL}$. The protein concentration of the $\mathrm{CM}$ in this experiment is unknown.

\section{The effect of OFM-cell CM on FB migration}

FB chemotaxis, using the agarose plug method, was measured using extracts of CM. The results are shown in Figure 4-4, along with a dose response to the chemokine PDGF. The cell count is the number of cells that have migrated to the central well, relative to the number of migrated cells in wells with media alone.

A

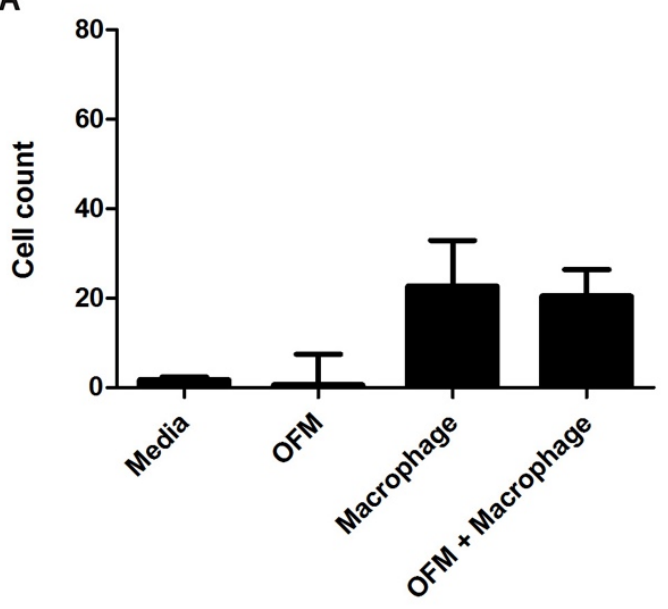

C

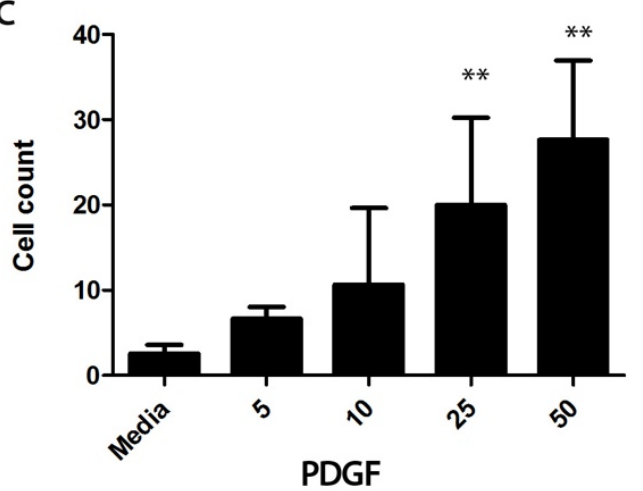

B

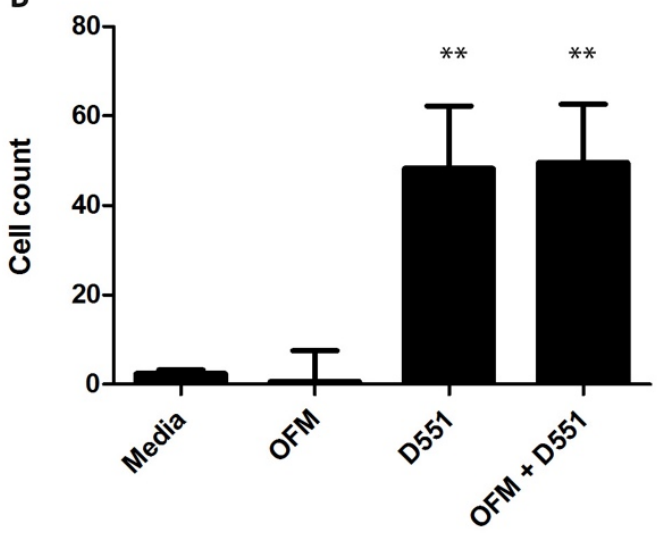

Figure 4-4 Chemotaxis of FBs in response to CM

(A) FB chemotaxis was tested using CM collected from OFM, macrophages and macrophages + OFM. No significant increase in chemotaxis was observed in macrophage and OFM + macrophage CM. (B) FB chemotaxis was also tested from CM 
collected from D551 and D551 + OFM CM, a significant increase is seen in both D551 and D551 + OFM CM. (C) FBs responded to growth factor PDGF (shown in ng/mL) in a dose dependent manner. Significant increase in chemotaxis was calculated by one-way ANOVA compared with media alone, ( $=6$ wells, ${ }^{* *} \mathrm{p}<0.05$ and $\left.{ }^{* * *} \mathrm{p}<0.001\right)$.

Figure 4-4 A shows there is a small increase in FB cell migration towards macrophage CM and macrophage-OFM CM. There is also significant migration towards FB CM and FBOFM CM, demonstrating that FB conditioned media is highly chemotactic to FBs. A dose response of FBs chemotaxis is observed using PDGF in the range of $0-50 \mathrm{ng} / \mathrm{mL}$. The combination of cells + OFM did not lead to a significant increase in cell motility in relation to the cell $\mathrm{CM}$ control, with regard to FB migration.

\section{The effect of OFM-cell CM on HUVEC migration}

Figure 4-5 shows the chemotaxis of HUVECs as tested using OFM CM, macrophage CM and macrophage + OFM CM (A), with VEGF as a control (B). HUVEC chemotaxis is stimulated by all three samples, as shown in (A). There is a significant difference in chemotaxis between the OFM + macrophage CM and the macrophage control, but not the OFM control. The activity in this sample could be due to the combination of OFM derived and macrophage derived factors within the sample.

A

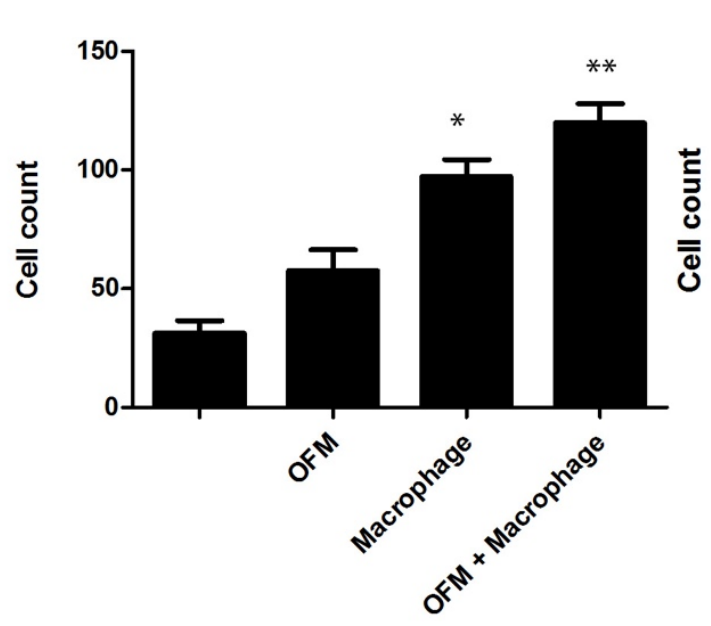

B

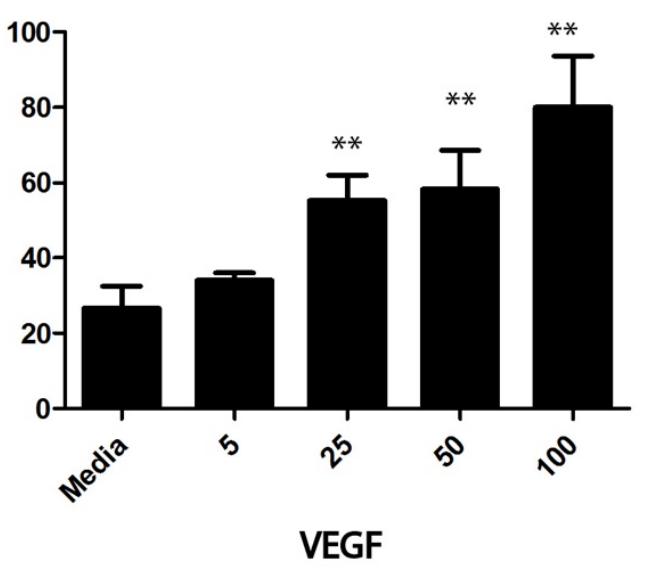

Figure 4-5 Chemotaxis of HUVECs in response to CM 
(A) HUVEC chemotaxis was tested using CM collected from OFM, macrophages and macrophages + OFM. (B) A VEGF control showed HUVECs respond to growth factor VEGF (shown in $\mathrm{ng} / \mathrm{mL}$ ) in a dose dependent manner. Significant increase in chemotaxis was calculated by one-way ANOVA compared with media alone, (n $=6$ wells, ${ }^{*} \mathrm{p}<0.05$ and $\left.{ }^{* *} p<0.001\right)$.

\subsubsection{SDS-PAGE separation of proteins in CM}

SDS-PAGE was used to separate proteins in the CM from different cultures. Concentrated CM samples, as well as controls of media alone are shown in Figure 4-6. Samples were separated in denaturing conditions without BME (lanes 1-6) and boiling for 5 minutes with BME (lanes 7 to 12). A sample of media, with FBS was included to demonstrate the amount of FBS in the CM.

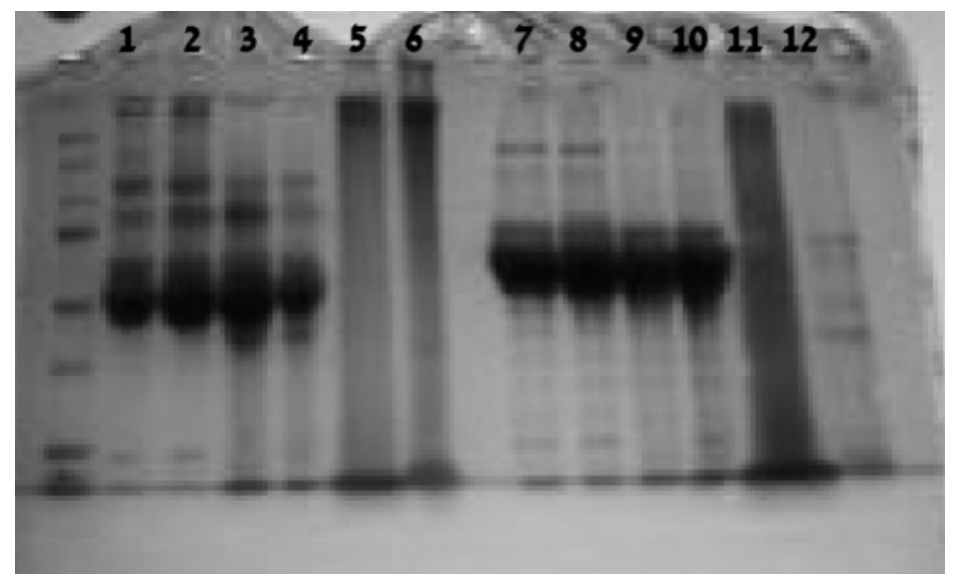

Figure 4-6 Proteins in CM separated by SDS-PAGE

CM samples were run on an SDS-PAGE gel, compared with pepsin digested samples of OFM without BME in denaturing conditions (lanes 1-6) and with BME in denaturing condition (lanes 7-12). A control of media with $10 \%$ FBS is shown in lane 1 and 7. CM samples with OFM only (lane 2 and 8), macrophages and OFM (lane 3 and 9), macrophages only (lane 4 and 10) and pepsin digested OFM (lane 5, 6 and 11).

The SDS-PAGE profiles of sample used in the assays shows that the majority of the protein content in all samples is the same as the media + FBS control (lane 1 and 7). No 
additional bands are clear in any of the CM samples. The pepsin digested OFM samples that were found to be bioactive in the previous chapter, appear to be a smear of differently sized degradation products (lanes 5,6 and 11).

\subsection{Discussion}

In Chapter Three, the digestion of OFM with pepsin led to a significant increase in the release of bioactives from OFM on wound healing cells, in terms of proliferation and migration. The non-specific digestion of OFM, or indeed any protein, with pepsin leads to an array of differently sized molecules, many of which could be responsible for the bioactivity. In fact, the SDS-PAGE gel in Figure 4-6 (lane 5, 6 and 11) shows that the pepsin digested sample is a heterogeneous mixture of OFM fragments. In this chapter, the possibility of using cells rather than enzymes was explored. Interestingly, specific combinations of cells and OFM have different activity on the migration of MSCs.

\subsubsection{Macrophage-mediated processing of OFM}

To determine whether the macrophages can digest OFM, OFM discs were stained with Congo red before cell seeding. Congo red binds non-covalently to the OFM, and is released into the media in a manner proportional to the number of macrophages seeded on the surface of the disc. A relatively low level of Congo red is released into the media without cells, indicating that the dye is leaching from the OFM, however this is greatly increased when macrophages are grown on the OFM. It appears that the macrophages cleaving labelled OFM proteins that are then coming into solution or cleaving bonds between the OFM and the Congo red. The images of cells after OFM seeding indicate that cells are phagocytosing Congo red stained OFM proteins. The amount of OFM or cell derived protein in samples of CM is very difficult to quantify due to the presence of $10 \%$ FBS in the pre-conditioning media. It is therefore difficult to measure the level of protein breakdown quantitatively using these methods. It is surprising that the macrophage proteolytic activity is not inhibited by the amount of FBS in the sample. 


\subsubsection{Bioactivity in $\mathrm{CM}$}

The relative bioactivity of OFM, cell and OFM-cell cultures was measured in relation to MSC, FB and HUVEC chemotaxis. Figure 4-3 shows that the combination of macrophage cells and OFM in culture gives a sample of CM that is chemotactic for MSCs. This sample of macrophage-OFM CM is effective at recruiting MSCs in a similar range to $7.5-10$ ng/mL PDGF (Figure 4-3 C). This effect is not seen in a sample of FB + OFM CM (Figure 4-3 B).

Interestingly, this effect was not observed when the same samples are tested for FB and HUVEC migration. These cells respond to the CM from both macrophages and FBs alone, there was no increase in chemotaxis when the cells were cultured with OFM. FBs are recruited by other FBs and macrophages during wound healing. In these experiments they did not appear to respond to OFM, or products of OFM liberated by these cells. The experiment on HUVECs shows that, macrophages alone and macrophages on OFM led to CM that is chemotactic for HUVECs, but there was no significant difference between these two.

This result is very encouraging since macrophages are known to promote ECM degradation in wound healing and are responsible for recruiting a number of cell lines. It is possible that macrophages break down $\mathrm{AECM}$ proteins that are then acting on other wound healing cells.

\subsubsection{Macrophages grown on OFM recruit MSCs in vitro}

The role of the macrophage in wound healing has been of great interest since the 1970s. It has been shown that macrophages release chemokines that recruit a number of different cell types to the wound site ${ }^{99,146,198}$. But why is there such a vast difference between the level of recruitment by macrophages alone, as opposed to macrophages grown on OFM? These are the most likely explanations for this observation: 
I. The increase in chemotaxis is the result of the combination of soluble OFM components $(\mathrm{O}-\mathrm{CM})$ and macrophage released chemokines $(\mathrm{M}-\mathrm{CM})$, in which case the activity of $(\mathrm{MO}-\mathrm{CM})=(\mathrm{O}-\mathrm{CM})+(\mathrm{M}-\mathrm{CM})$

II. The macrophage cells change their phenotype in response to being grown on or attached to the OFM, resulting in change in the expression of MSC-specific chemokines

III. The increase in chemotaxis is due to the release of OFM components by the macrophage cells

In theory, I. can be tested empirically using a ratio of means. In Table 4-1, M-CM and O$\mathrm{CM}$ activity is expressed as a fraction of the activity of the MO-CM in relation to MSCs, FBs and HUVECs. For the MSC cell line, the combined ratio of means of the two control samples, $\mathrm{M}-\mathrm{CM}$ and $\mathrm{O}-\mathrm{CM}$, does not equal the activity of the MO-CM sample, over three independent experiments. The combined ratios (0.47) are less than half of the activity of the MO-CM (1). When this calculation is applied to bioactivity of FB and HUVECs, the combination of $\mathrm{M}-\mathrm{CM}$ and $\mathrm{O}-\mathrm{CM}$ is greater than the activity of the MO-CM sample. The number of replicates in each experiment ( $r$ ) and the number of independent experiments (n) is shown for each cell type.

\begin{tabular}{llll} 
& MSC & FB & HUVEC \\
& $(\mathrm{N}=3, \mathrm{R}=5)$ & $(\mathrm{N}=1, \mathrm{R}=5)$ & $(\mathrm{N}=2, \mathrm{R}=5)$ \\
\hline M-CM & $0.22(+/-0.07)$ & 0.82 & $0.67(+/-0.24)$ \\
O-CM & $0.24(+/-0.07)$ & 1.02 & $0.91(+/-0.01)$ \\
M-CM AND O-CM & 0.47 & 1.84 & 1.58 \\
MO-CM & 1 & 1 & 1
\end{tabular}

Table 4-1 Calculation of CM bioactivity as ratio of means

In the case of II., the assumption is that the presence or absence of proteases in the MOCM sample is irrelevant. In this case, the proximity of OFM proteins or molecules elicits a response modifying the chemokine profile expressed by the macrophages. Cells respond to a physical change in their surroundings by signalling through integrins. In this scenario, the macrophages are behaving in the same way that keratinocytes respond to the change in 
physical contact by recruiting other cells. If this is the case, OFM and other materials may promote wound healing by direct interaction with host cells. One way to test this would be to compare the real time polymerase chain reaction (rtPCR) and protein expression profile of the cells in the M-CM and MO-CM samples, in order to determine changes in cytokine or growth factor expression. Another possibility is the change from Mo to M1 or M2 phenotype caused by the presence of the OFM. Resting macrophages appear rounded in culture compared with "polarised" active macrophages. This activation results in either an M1 or an M2 phenotype, which can be determined by their cytokine profile.

The effect of macrophage phenotype as a result of OFM proteins would be highly relevant to the wound healing process. Other studies have shown that dECMs often promote an M2 phenotype during wound healing ${ }^{199,199}$. Discovering the dECM proteins that promote this phenotype could be very useful in biomaterial design.

An observation was made with regard to the question of macrophage polarisation during this study. Macrophages incubated with the various OFM extracts and CMs showed that pepsin digested OFM led to clearly visible changes in the macrophages culture. Control samples showed rounded macrophages, with few extended processes, however incubating cells with pepsin digested OFM led to highly polarised macrophages, with extended processes (Figure 4-7). This observation does show that OFM proteins may modify the phenotype of macrophages in some way, but it does not show whether their phenotype is M1 or M2. 

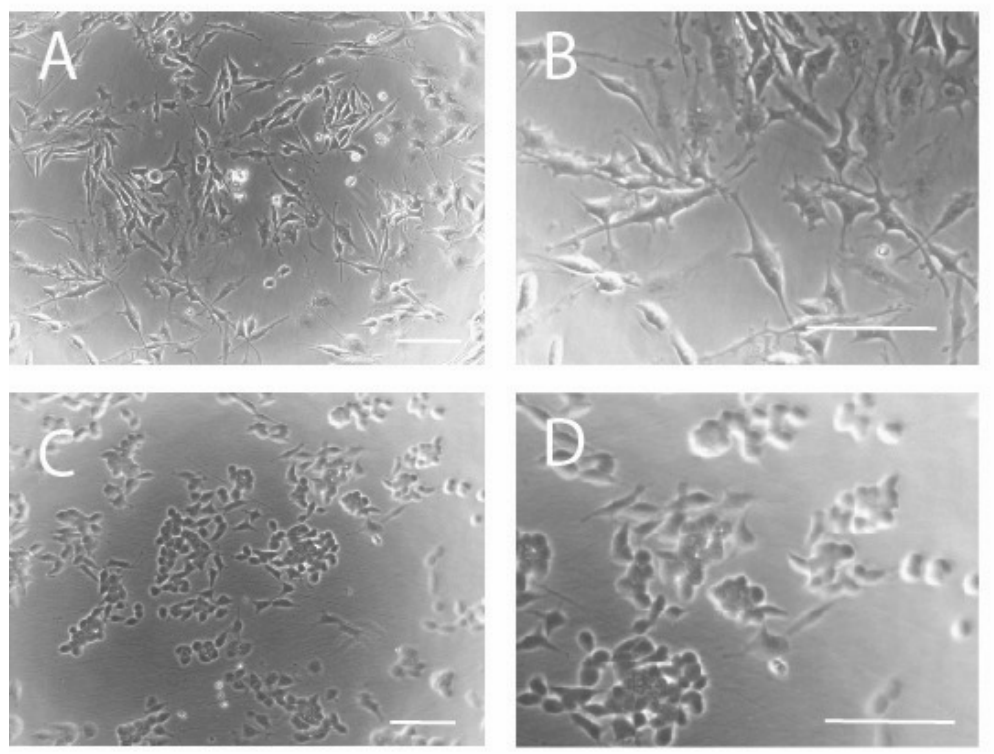

\section{Figure 4-7 Macrophage polarisation by OFM proteins}

Pepsin-digested OFM was incubated with macrophages overnight a (A and B).

Compared with normal media (C and D). Cells incubated with pepsin digested OFM appear to have more processes per cell. Scale bar (white) is $50 \mu \mathrm{m}$.

Finally, assuming (iii.) to be true, that macrophages are releasing bioactive molecules in the same way that pepsin digesting the OFM released bioactivity, then it is plausible that the OFM contains matricryptins that are chemotactic for cells in vitro. These could be used to promote wound healing if they can be isolated and identified from the samples. As shown in Figure 4-6, the samples separated by SDS-PAGE show no distinct or novel bands, due to the presence of large amounts of FBS in the sample.

\subsubsection{Interference of FBS in the culture system}

A major limitation to these experiments is the requirement to use FBS as a supplement in many cell culture systems. Cells such as the MSCs are able to grow slowly without FBS, while HUVECs and FB will not. In addition, the OFM must be pre-conditioned in media containing serum in order for cells to attach to the ECM.

This presents several major challenges: 
I. The FBS itself contains many bioactive molecules that alter cell migration and proliferation. In some migration assays, FBS is used as a control chemo-attractant.

II. FBS inhibits the action of many proteases, although the Congo red release assay shows that macrophage proteases were still able to digest collagen in culture despite FBS carry over from the pre-conditioning step.

III. The presence of $10 \%$ FBS makes any additional proteins bands impossible to see by SDS-PAGE, and will undoubtedly interfere with the isolation and identification of macrophage or OFM derived proteins.

IV. FBS may bind to Congo red or other labels used to identify OFM proteins from macrophage proteins.

\subsection{Conclusions}

The evidence presented in this chapter suggests that the macrophages may release proteases that breakdown the OFM into bioactives in the same way that pepsin digested samples of OFM contained bioactives. From these experiments, it is clear that the combination of macrophages and OFM in culture (the MO-CM sample) generates a solution that is chemotactic for MSCs. Importantly, this increase in the chemotactic potential of the CM is not observed using HUVECs and FBs. Although macrophages alone and OFM alone generate MSC chemo-attractant $\mathrm{CM}$, it is clear that MSC chemotaxis is significantly increased when macrophages are grown on OFM. The release of Congo red by macrophages from pre-stained OFM support the theory of cell-mediated digestion of OFM. Alternatively, OFM may be directing the behaviour of the macrophages to express chemokines; this requires further substantiation using rtPCR to profile the protein expression in $\mathrm{M}-\mathrm{CM}$ and $\mathrm{MO}-\mathrm{CM}$. 
Extracellular matrix derived peptides and mesenchymal stem cell motility Sandi Grainne Dempsey - September 2016 


\section{Macrophage-mediated release of an ECM derived protein}

\subsection{Summary}

In the previous chapter, the bioactivity of $\mathrm{CM}$ collected from cultures of cells grown on OFM was investigated. The combination of macrophages and OFM generated CM that was chemotactic for MSCs in vitro. It was not established whether this was due to the cellmediated processing of the OFM, or a change in macrophage cytokine expression as a result of the OFM in culture. This chapter presents the attempts made to label the OFM as a means of distinguishing OFM derived proteins from macrophage derived proteins within the CM.

\subsection{Introduction}

Macrophages play numerous roles in wound healing and express a number of proteases that are important in this process ${ }^{146,198}$. The presence of these cells at the periphery of the wound is indicative of a healthy, regenerative wound response; particularly after a phenotypic switch to an M2 phenotype as opposed to a pro-inflammatory M1 phenotype. Macrophage-OFM interactions have not been previously studied, however there are many instances where the presence or absence of these cells during biomaterial-mediated wound healing have been alluded to in the literature. Bullers et al. demonstrated that macrophages seeded onto a decellularised bladder matrix resulted in polarisation of the macrophages, indicated by the expression of certain markers, in particular nuclear associated peroxisome proliferator-activated receptor gamma $(\operatorname{PPAR} \gamma)^{200}$. They concluded that biomaterial associated factors lead to active recruitment and polarisation of local macrophages and 
contributed to the constructive modelling process. Furthermore they suggested that the infiltration of dECMs in vivo is initiated by macrophages. Another study demonstrated distinct responses of macrophages to the presence of deacetylated chitosan compared with chitin; the former was found to illicit a greater inflammatory response, as measured by the expression of IL-1 $\beta$ and NLRP3 leading to a greater phagocytosis response compared with chitin, which was inert ${ }^{201}$. Ariganello et al. compared the macrophage response to decellularised bovine pericardium (DBP) with that of polystyrene ${ }^{199,202}$. After 14 days, macrophages cultured on these materials expressed high levels of the protease MMP-9, and low levels of MMP-2 and MMP-1. The DBP associated macrophages expressed more MMP-2 and less acid phosphatase. They also expressed higher levels of IL-6 and IL-8 and lower levels of IL-10 IL-1ra. This led the authors to conclude that the DBP associated macrophages were not expressing an activated inflammatory response comparable to macrophages grown on polystyrene.

In this study it has been shown that macrophages grown on OFM led to the release of chemotactic CM. If macrophages were stimulated by OFM to express cytokines that recruit other cells, this would be an important finding in relation to the bioactivity of OFM as a promoter of wound healing. It is also possible that the macrophages are breaking down the OFM, releasing chemotactic fragments from the ECM. The Congo red release assay demonstrated that macrophages in culture express ECM degrading proteases. Cells can mediate the release of bioactive proteins and peptides from the ECM by proteolytic activity. For example, MT1-MMP and MMP-2 cleave EGF-like repeats from laminin 5; elastase and cathepsins release endostatin from collagen; MMP-9 releases tumstatin from collagen IV and MMP-2, MMP-9, MMP-7 and MMP-12 release the XGXXPG consensus of elastin ${ }^{88,89}$. These matricryptins have distinct biological activity from their parent protein. It is possible that the macrophage mediates the breakdown of OFM leading to the release of OFM derived factors or matricryptins.

\section{Methods of protein labelling}


One way to identify the origin of the proteins in solution is to covalently label the OFM before culture with macrophage cells. In choosing a label for this study, a number of factors were considered. Firstly, OFM labelling should not affect the ability of the macrophages to attach to OFM. Secondly, labelling should not prevent protease digestion by masking binding sites on OFM proteins. Thirdly, the labelling must not cause cell death in the culture. Finally, the CM sample was already active, labelling the OFM should not induce changes in the proteins that render them inactive, by interfering with active sites for instance.

Two useful protein labels are biotin-avidin conjugates and fluorescent probes. Biotin is useful for protein labelling because it is small (244 Da) and forms a very strong noncovalent link with avidin. Biotin labelled (or biotinylated) proteins can be separated by incubating a sample with streptavidin beads and unlabelled proteins are washed away. Fluorescent probes are very versatile protein labels, they are also small (FITC is 389 Da) and require no addition reagents for detection. FITC contains an isothiocyanate reactive group that binds to amine and sulfhydryl groups on proteins.

One of the disadvantages of biotin labelling is that separation of the biotin and avidin requires harsh conditions that can denature the protein. Consequently, OFM was labelled using FITC so that labelled peptides could be easily identified in an SDS-PAGE gel using a fluoroscanner. In theory, the isolation of fluorescent fractions after separation of proteins by column filtration or other methods could be quickly measured using a fluorescent plate reader. Also, since labelled and unlabelled samples could be isolated side by side using the fluorescent samples as a trace, this would give a purified unlabelled sample that has not been altered by the labelling method.

\subsection{Aims}

MO-CM is chemotactic for MSCs. The CM itself contains FBS proteins, OFM proteins and macrophage proteins. The aim of this section was to remove as much contaminating FBS as possible and determine whether MO-CM contained additional proteins compared with $\mathrm{M}-\mathrm{CM}$ and $\mathrm{O}-\mathrm{CM}$ and whether they were derived from the OFM or the macrophages. 


\subsection{Results}

In this chapter, the collection of $\mathrm{CM}$ was altered to enable identification of discrete peptides from a macrophage or OFM source. Labelled and unlabelled cultures and CM in this chapter will be described in the text as follows:

- M-CM: macrophage conditioned media

- O-CM: OFM conditioned media

- $\mathrm{O}_{\mathrm{F}}-\mathrm{CM}$ : FITC labelled OFM conditioned media

- MO-CM: macrophage-OFM culture conditioned media

- $\mathrm{MO}_{\mathrm{F}}-\mathrm{CM}$ : macrophage + FITC labelled OFM culture conditioned media

\subsubsection{Macrophage uptake of FITC labelled protein}

Macrophages grown on FITC labelled OFM were removed and imaged under a fluorescent microscope. Macrophages were scraped from $\mathrm{MO}_{\mathrm{F}}-\mathrm{CM}$ and $\mathrm{MO}-\mathrm{CM}$ cultures and imaged at $40 \mathrm{x}$ and $100 \mathrm{x}$ magnification. Cells grown on labelled $\mathrm{OFM}\left(\mathrm{MO}_{\mathrm{F}}\right)$ appear fluorescent while those from unlabelled OFM are not visible, as shown in Figure 5-1. In the 100x magnified images (D and E), fluorescent clusters appear within the cells. 

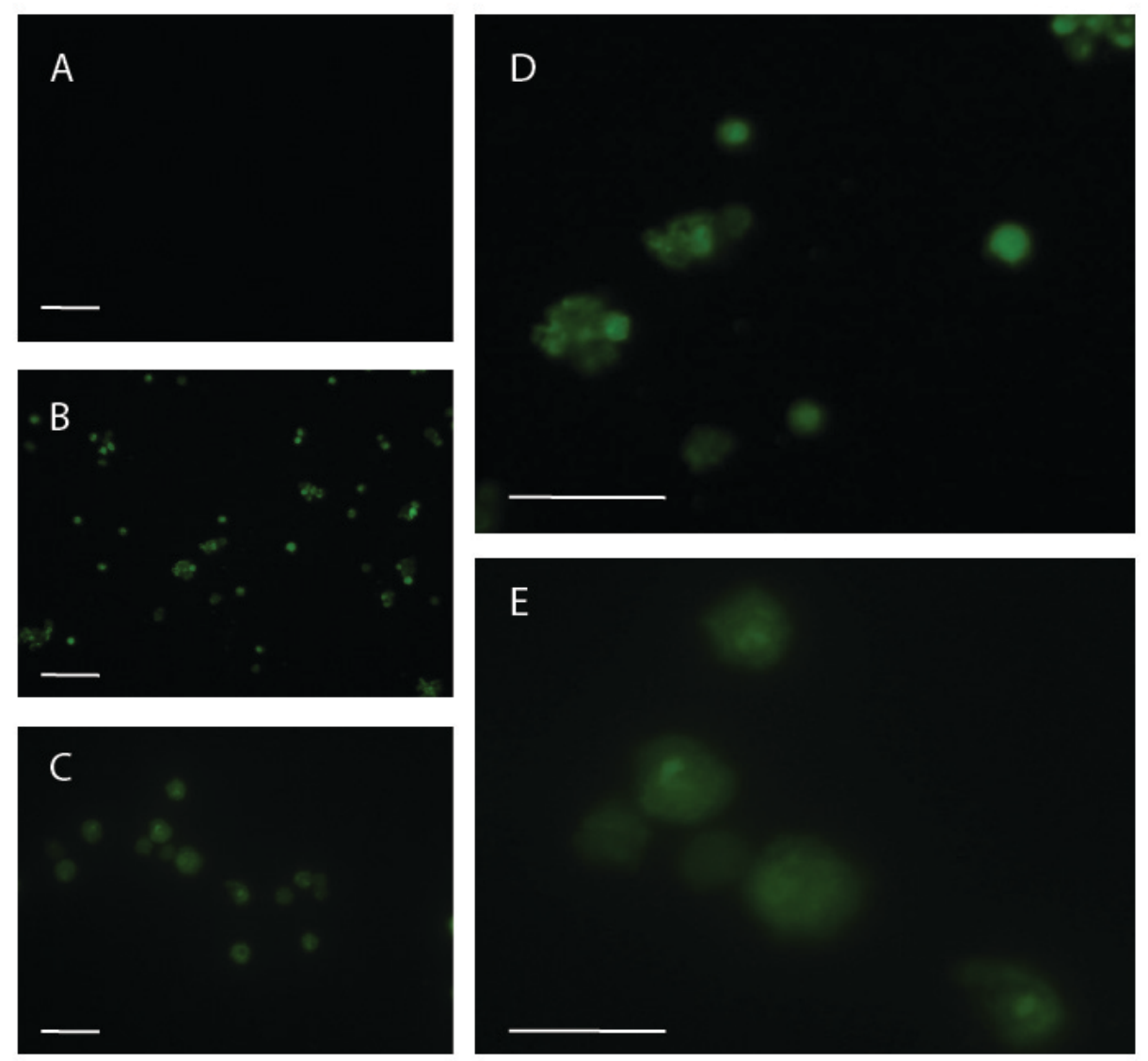

Figure 5-1 FITC uptake by macrophages grown on $\mathrm{OFM}_{\mathrm{F}}$

(A) Macrophages at 40x are not visible after seeding on unlabelled OFM. Macrophages grown on FITC labelled OFM appear fluorescent at 40x (B) and 100x (C). Cropped images of 40x (D) and 100x (E) cells show that intracellular fluorescent clusters appear. Scale bars (white) are represented in A and B: $50 \mu \mathrm{m}, \mathrm{C}: 20 \mu \mathrm{m}, \mathrm{D}: 25 \mu \mathrm{m}$ and E: 10 $\mu \mathrm{m}$.

\subsubsection{SDS-PAGE analysis of FITC labelled protein}

Samples of CM were separated using a $20 \%$ SDS-PAGE gel and scanned using a FITC filter on a flouroscanner to identify labelled peptides in the conditioned media. The gel was then stained with Coomassie to show all proteins in the sample. Figure 5-2 (A) shows 
the fluoroscan gel, and (B) shows the same gel, after Coomassie staining. Samples in this gel are:

1. $\mathrm{MO}_{\mathrm{F}}-\mathrm{CM}$

2. $\mathrm{M}-\mathrm{CM}$

3. $\mathrm{MO}-\mathrm{CM}$

4. $\mathrm{O}_{\mathrm{F}}-\mathrm{CM}$

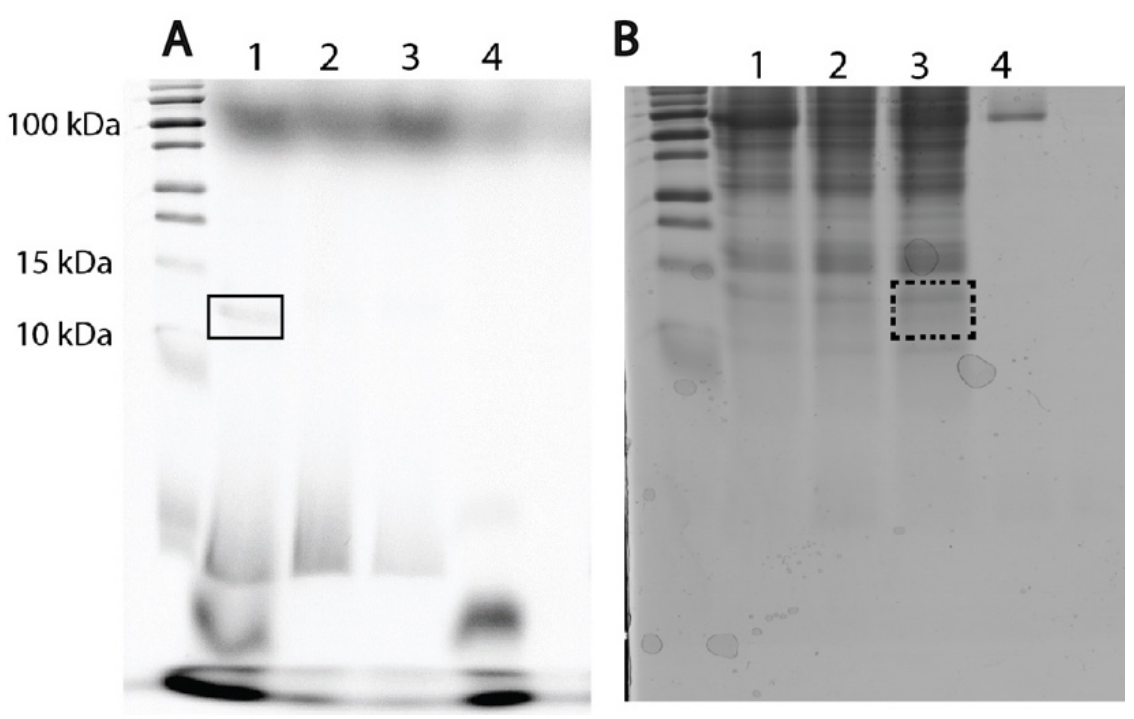

Figure 5-2 Labelled and unlabelled CM on $20 \%$ SDS-PAGE

Labelled and unlabelled CM samples were separated by SDS-PAGE. 1- $\mathrm{MO}_{\mathrm{F}}, 2-\mathrm{M}, 3-$ MO (unlabelled) and 4- $\mathrm{O}_{\mathrm{F}}$ (no cells). (A) Shows the pre-stained gel imaged using a fluoroscanner under a FITC channel, showing only fluorescently labelled proteins. Gel (B) show all proteins in sample after Coomassie staining.

In Figure 5-2 (A) a distinct fluorescent band is seen in lane $1\left(\mathrm{MO}_{\mathrm{F}}-\mathrm{CM}\right.$ sample), between 10 and $15 \mathrm{kDa}$ (inside box). This band is not present in any of the other samples and is referred to as the "band of interest" from here on. Lanes 1 through 3 also contain a band of large FITC labelled protein $(>100 \mathrm{kDa})$. All four lanes also contain a band or bands of labelled proteins that are less than $5 \mathrm{kDa}$. Bright bands seen in lane 1 and 4 (less than 5 $\mathrm{kDa}$ ) may be the unbound FITC which has come into solution.

Figure 5-2 (B) shows the same gel after Coomassie staining, which binds to all proteins in the sample. In lanes 1-3 ( $\mathrm{MO}_{\mathrm{F}}-\mathrm{CM}, \mathrm{M}-\mathrm{CM}$ and $\left.\mathrm{MO}-\mathrm{CM}\right)$ contain a large number of differently sized proteins, these are not seen in lane $4\left(\mathrm{O}_{\mathrm{F}}-\mathrm{CM}\right)$, which contains a single 
high $\mathrm{Mw}$ band (>100 $\mathrm{kDa}$ ). This indicates that proteins in lanes 1 to 3 are derived from macrophage CM. In this gel it is impossible to distinguish the protein of interest in lane 1 , from other macrophage proteins in lane 2 and 3. The single high $\mathrm{Mw}$ band in lane $4\left(\mathrm{O}_{\mathrm{F}^{-}}\right.$ $\mathrm{CM}$ ), is probably OFM proteins which have come into solution during culture.

Comparing the two gels, it is clear that the band of interest in lane 1 (A) is not the only protein of this size in the sample, as shown in the Coomassie stained gel lane 1 (B).

The samples in the dotted box containing unlabelled OFM peptides and macrophage peptides. This section of the gel was excised for MALDI analysis, described in the next chapter.

\subsubsection{Improving resolution of SDS-PAGE separation}

In order to improve the resolution of the fluorescent peptides in the sample, the ratio of cells to OFM was decreased using a larger $(4 \times 4 \mathrm{~cm})$ piece of OFM with the same number of cells and a larger volume of culture media. The culture media used was phenol red free DMEM with no serum. Samples were separated on a tricine gel to improve resolution of smaller peptides. Figure 5-3 shows the fluoroscan (A) and Coomassie stained gel (B). Undiluted samples (4 to 6) and concentrated ( $5 \mathrm{x}$ ) samples (1 to 3) are shown in lanes:

1. $\mathrm{MO}_{\mathrm{F}}-\mathrm{CM}$ (concentrated)

2. $\mathrm{MO}-\mathrm{CM}$ (concentrated)

3. $\mathrm{M}-\mathrm{CM}$ (concentrated)

4. $\mathrm{MO}_{\mathrm{F}}-\mathrm{CM}$ (undiluted)

5. MO-CM (undiluted)

6. M-CM (undiluted) 
A

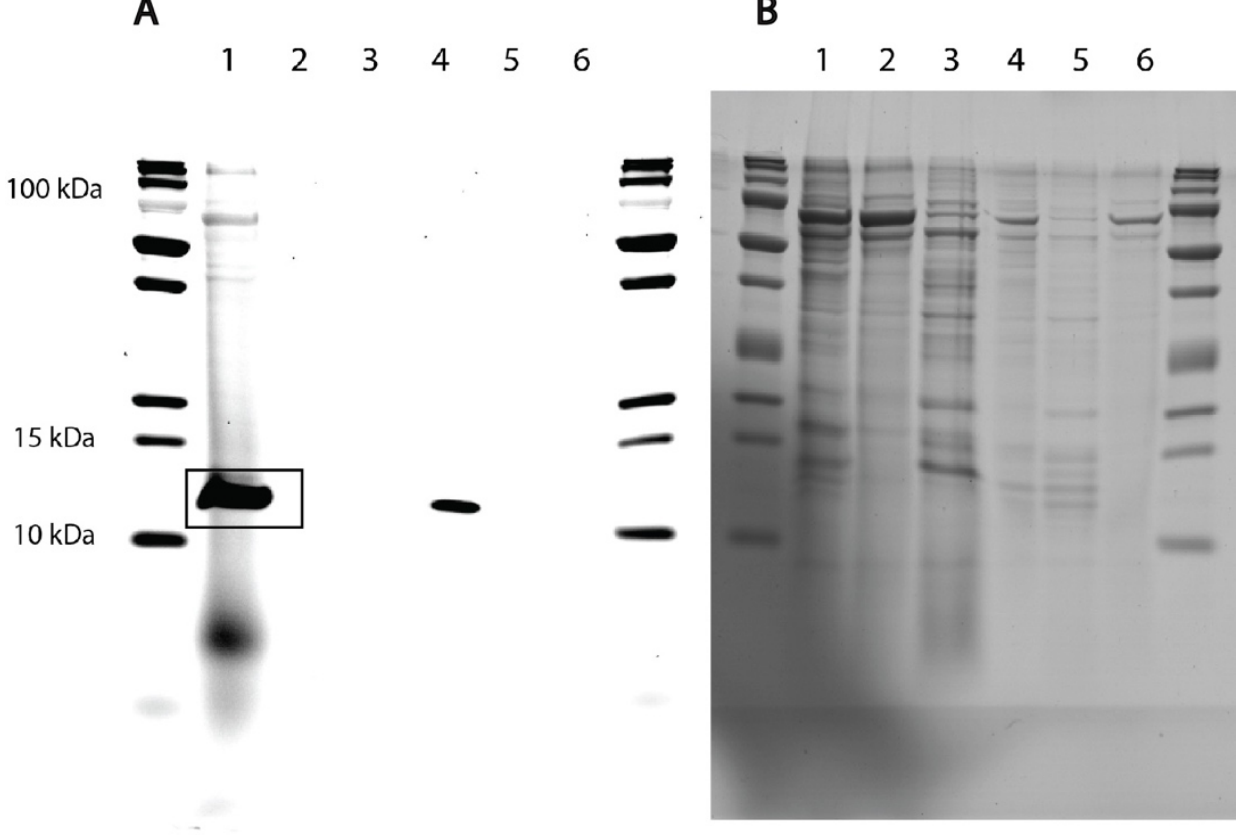

Figure 5-3 Labelled and unlabelled CM on $16 \%$ Tricine SDS-PAGE

Samples in lane 1- $\mathrm{MO}_{\mathrm{F}}$, 2- MO, 3- M, 4- $\mathrm{MO}_{\mathrm{F}}$, 5- MO, 6- M. Samples 4 through 6 are undiluted samples of CM, while samples 1-3 have been concentrated five fold. Gel images show the fluoroscan on the left and after Coomassie staining on the right. Coomassie stained gel (right) shows all the proteins in the sample, while the FITC scanner shows only those that appear fluorescent (OFM derived).

The fluorescent band between 10 and $15 \mathrm{kDa}$ in lane 1 and 4 in this gel is much more prominent than the large band $(>100 \mathrm{kDa})$ and the smaller band. This indicates that the peptide of interest is enriched in this sample, compared with Figure 3-2. In the Coomassie stained gel, note that there are a large number of proteins in the M-CM sample in lane 3 (macrophages alone) which correspond to bands in the $\mathrm{MO}-\mathrm{CM}$ and $\mathrm{MO}_{\mathrm{F}}-\mathrm{CM}$ samples (lane 1 and 2). It is difficult to identify proteins which are unique to the MO-CM sample (lane 2) compared with the M-CM sample (lane 3), with the exception of a large band around $100 \mathrm{kDA}$ contained in both $\mathrm{MO}-\mathrm{CM}$ and $\mathrm{MO}_{\mathrm{F}}-\mathrm{CM}$. The bands in the $\mathrm{MO}-\mathrm{CM}$ (lane 2) sample appear slightly fainter than the $\mathrm{MO}_{\mathrm{F}}-\mathrm{CM}$ sample (lane 1). 


\subsubsection{Separation by DEAE}

The fluorescent sample was enriched using a DEAE batch preparation protocol. The samples were separated by SDS-PAGE shown in Figure 5-4 as undiluted samples (lane 1 through 5) and concentrated samples (lane 6 through 10). Lane 1 and 6 shows the initial sample of $\mathrm{MO}_{\mathrm{F}}-\mathrm{CM}$, undiluted and concentrated respectively. Lane 2 and 7 shows the unbound proteins which were removed into the buffer $(10 \mathrm{mM}$ tris $\mathrm{pH} 8.3)$ after incubating the samples with DEAE beads. Lanes 3, and 8 shows sample of buffer after rinsing the DEAE beads. Lanes 4 and 9 show the proteins eluted from the DEAE beads into buffer by $150 \mathrm{mM} \mathrm{NaCl}$. Lanes 5 and 10 show proteins eluted from the DEAE beads into buffer by $400 \mathrm{mM} \mathrm{NaCl}$.

The fluoroscan shows that a distinct fluorescent band is present in the initial sample (lane 1) and enriched in lane 4 and 5, while the majority of other proteins are lost in the wash solution (lanes 2 and 7). Note that although the fluorescent band can be observed in lane 4, 5,8 and 9 , these bands do not appear in the Coomassie stained gel.

A
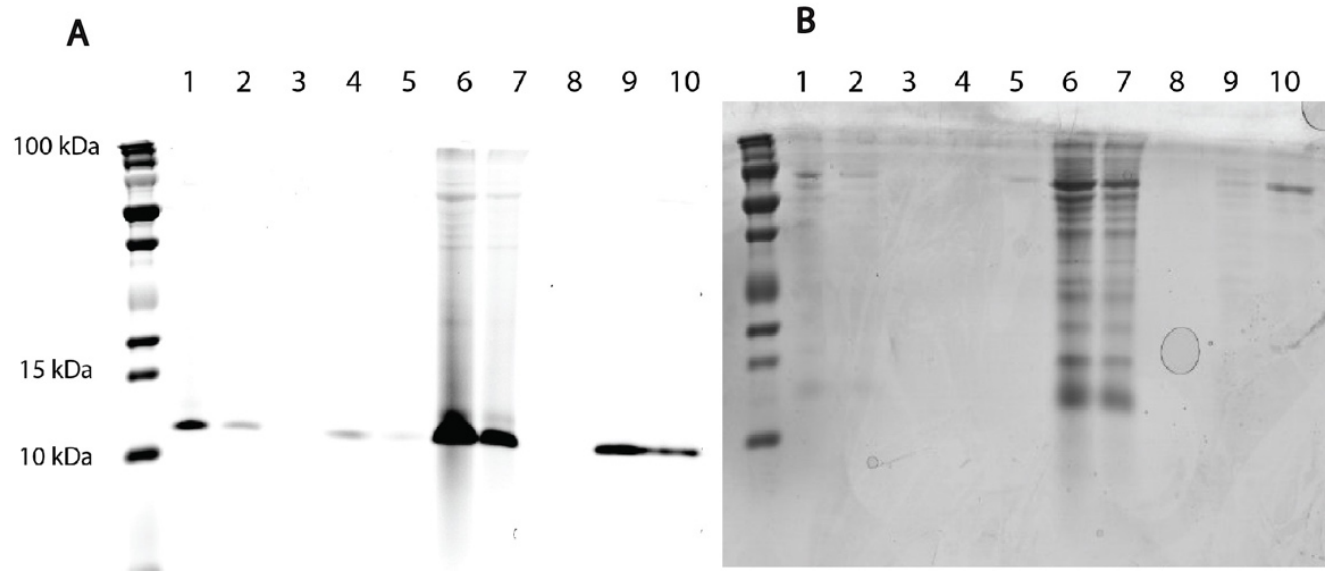

Figure 5-4 Batch separation of DEAE bound fractions

Fluoroscan (A) and Coomassie stained (B) tricine gel of samples after DEAE batch separation. Lane 1 to 5 are undiluted samples and lane 6 to 10 have been concentrated 5x. Lane 1 is the initial sample, lane 2 is the wash buffer (tris $10 \mathrm{mM}$ ), lanes 3 to seven are peptides removed from DEAE beads with increasing salt concentration 
ImageJ was used to calculate the area in each band from Figure 5-4 to estimate the enrichment of the $12 \mathrm{kDa}$ protein of interest in the DEAE separated sample. The lanes were plotted to give peaks and the area under the curve was measured. The data in Table 5-1 shows that the area under the peak in the FITC labelled protein decreased from lane 1 to the separated samples (lane 2, 4 and 5). However, the ratio of the fluorescent protein compared with the total protein measured in the Coomassie stained gel increased in lane 4 by four fold, compared with the original sample (lane 1).

\begin{tabular}{lll}
$\begin{array}{l}\text { FLUORESCENT PROTEIN } \\
\text { (AREA UNDER CURVE) }\end{array}$ & $\begin{array}{l}\text { TOTAL PROTEIN } \\
\text { (AREA UNDER CURVE) }\end{array}$ & RATIO \\
\hline 4532.698 & 43677.361 & 0.10 \\
895.263 & 24369.278 & 0.04 \\
961.678 & 2301.054 & 0.41 \\
202.435 & 8899.806 & 0.02
\end{tabular}

\section{Table 5-1 Ratio of F protein band to total protein}

The DEAE separation was scaled up to a step gradient column separation, in order to increase the yield of the protein of interest. Fractions were collected from the DEAE column and separated by SDS-PAGE (Figure 5-5). Lane 1 is the initial sample $\left(\mathrm{MO}_{\mathrm{F}}-\mathrm{CM}\right)$ and lanes 2 through 6 are fractions collected with an increasing ionic concentration. The fluorescent peptide is visible in lanes 5 and 6 of the fluoroscan (A), however these bands do not appear in the Coomassie stained gel (B). Lane 7 shows the unlabelled unseparated sample (MO-CM). This sample shows a similar profile to the labelled sample (lane 1) in the Coomassie stained gel (B). A faint band is shown in lane 7 around $12 \mathrm{kDa}$, which may correspond to the fluorescently labelled protein of interest in lane 1. 

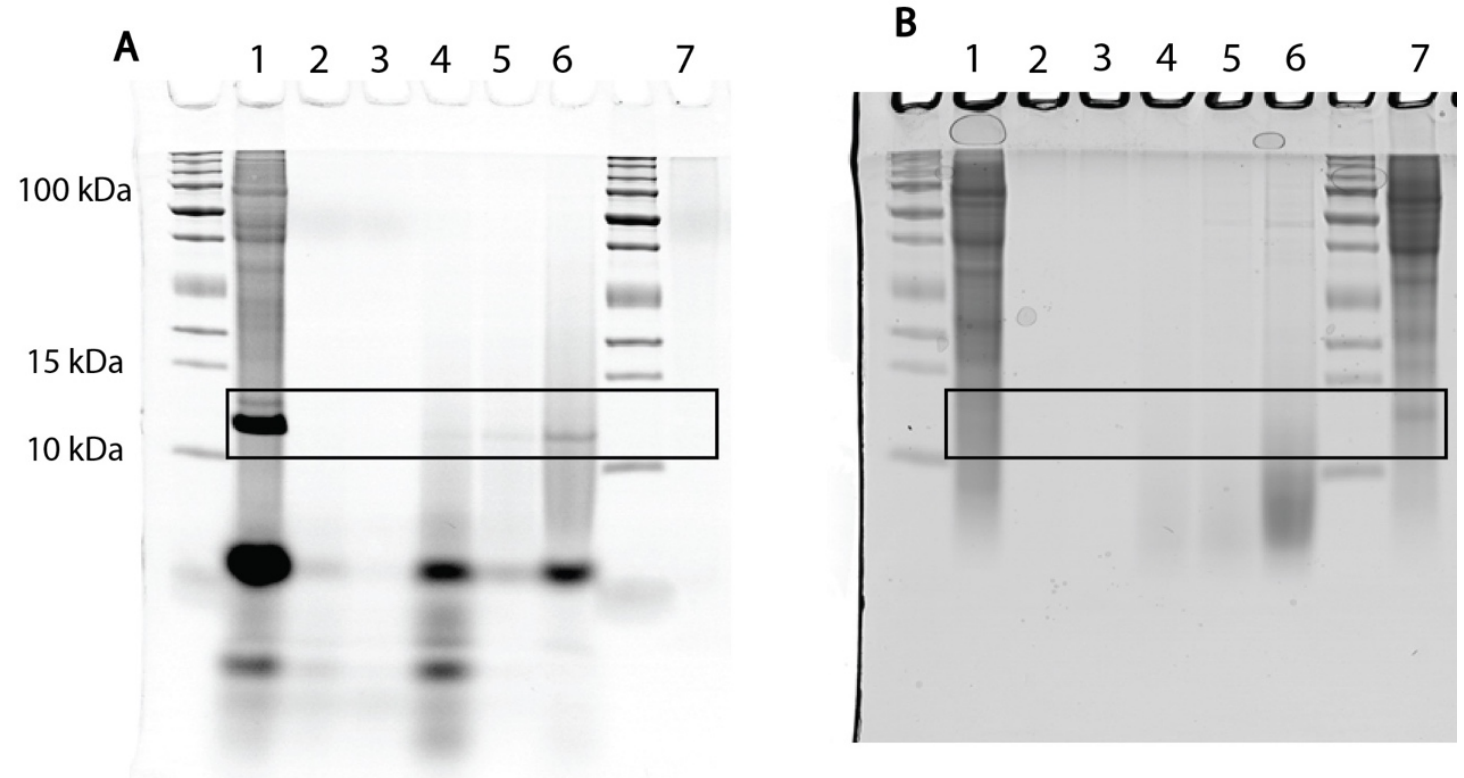

Figure 5-5 DEAE step gradient column separation of $\mathrm{MO}_{\mathrm{F}}$ sample

Lane 1 (initial sample of $\mathrm{MO}_{\mathrm{F}}-\mathrm{CM}$ ) and lanes 2 to 6 are fractions collected with increasing anionic strength. Fluoroscan (A) and Coomassie stained (B) show enrichment of the fluorescent peptide in relation to the total protein in the sample. Lane 7 shows the unlabelled sample of MO-CM.

DEAE separation of the peptide resulted in a loss in yield. The Coomassie stained gel shows that the peptide is not visible in these lanes. FITC labelled proteins are more sensitive to visualisation than Coomassie stained proteins. Coomassie stained proteins are visible at a protein concentration greater than $100 \mathrm{pM}$. The presence of the OFM derived peptide in the fluoroscan but not the Coomassie stained gel indicates that the concentration of the OFM derived peptide is below the detectable limit for Coomassie.

The culture experiment was scaled up to give $50 \mathrm{~mL}$ of $\mathrm{CM}$ samples $\mathrm{MO}_{\mathrm{F}}-\mathrm{CM}$ and $\mathrm{MO}-$ CM. These samples were concentrated to less than $5 \mathrm{~mL}$ and separated individually using the DEAE column filtration set up and collected in $1 \mathrm{~mL}$ fractions. The protein concentration and fluorescence of each collected fraction was measured and traced as shown in Figure 5-6. A fluorescent peak was observed in fractions 7 through 11 which corresponded to a protein concentration peak in the $\mathrm{MO}_{\mathrm{F}}-\mathrm{CM}$ sample (A). This peak was mirrored in the unlabelled MO-CM sample (B). The fractions collected in tubes 7, 8, 9, 10 
and 11 were pooled for each sample giving a final protein concentration of $0.3 \mathrm{mg} / \mathrm{mL}$ of the enriched protein of interest from $\mathrm{MO}-\mathrm{CM}$ and $0.25 \mathrm{mg} / \mathrm{mL}$ enriched protein of interested from $\mathrm{MO}_{\mathrm{F}}-\mathrm{CM}$. The unlabelled protein of interest enriched from the MO-CM was labelled "peptide A".
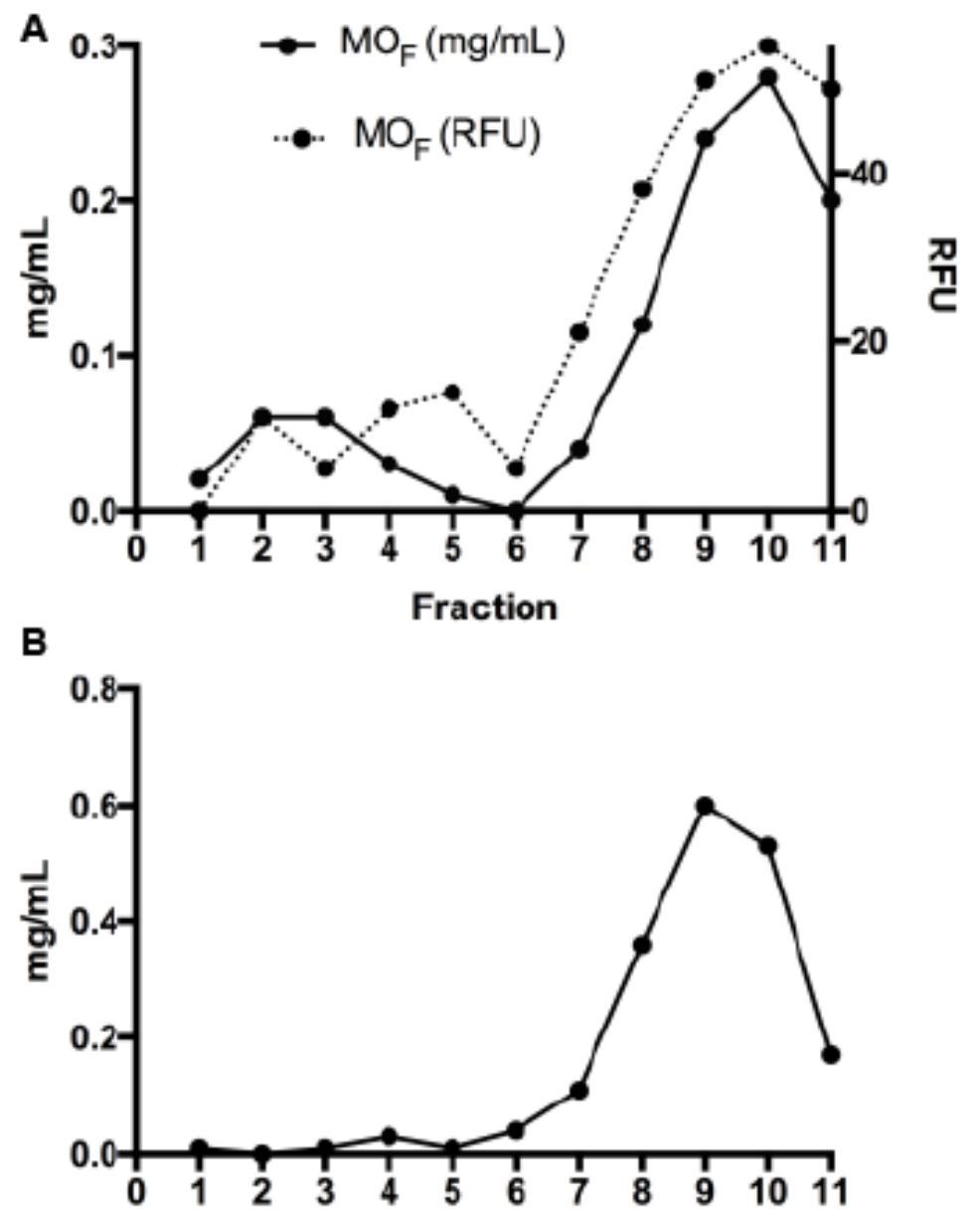

Fraction

Figure 5-6 DEAE samples collected for peptide A

MO-CM and $\mathrm{MO}_{\mathrm{F}}-\mathrm{CM}$ samples were collected from a DEAE column in $1 \mathrm{~mL}$ fractions. The protein concentration and and RFU were measured for each fraction collected from the $\mathrm{MO}_{\mathrm{F}}-\mathrm{CM}$ sample (A). The protein concentration was measured for each fraction collected from the MO-CM sample (B). Fractions 7 through 11 were collected from MO-CM and this protein was labelled peptide A, an enriched and unlabelled solution of the protein of interest. 


\subsubsection{MSC chemotaxis using enriched peptide A}

The MSC chemotaxis assay used for MO-CM (Chapter Four) was repeated using the enriched protein of interest, peptide A with 10 ng PDGF as a control. Peptide A in the chemotaxis assay was measured in a range of 0 and $100 \mathrm{ng}$. The cell count in the chemotaxis assay was significantly higher with a concentration of $10 \mathrm{ng}, 25 \mathrm{ng}$ and $100 \mathrm{ng}$, as shown in Figure 5-7.

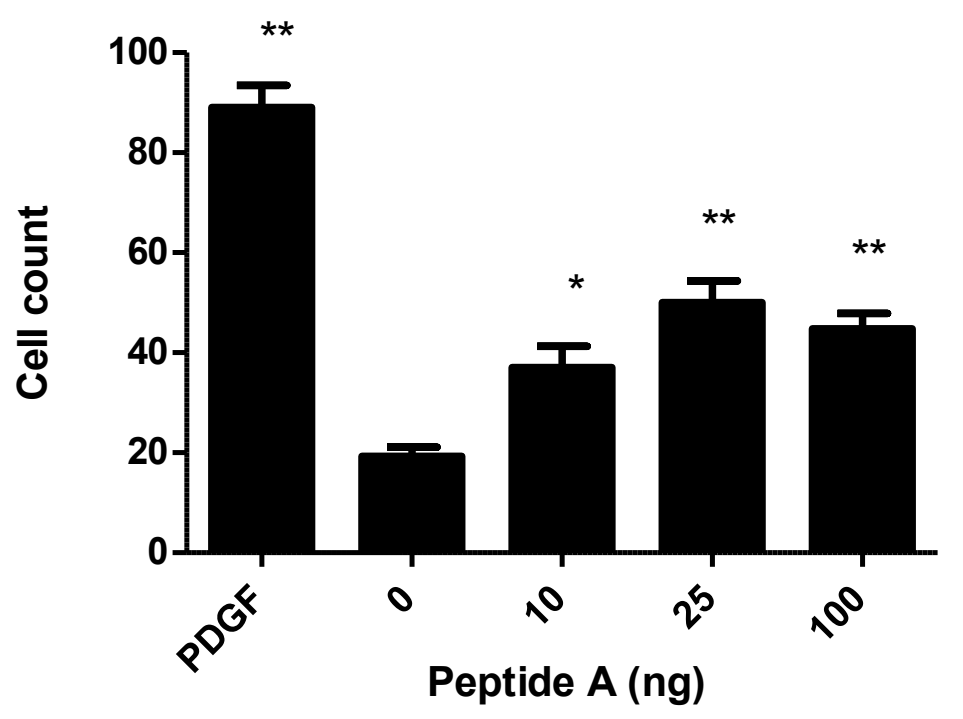

Figure 5-7 Chemotaxis of enriched fraction of peptide A

MSC chemotaxis was measured using the enriched sample of peptide using $10 \mathrm{ng}, 25$ $\mathrm{ng}$ and $100 \mathrm{ng}$ of the enriched peptide A. Peptide A showed a significant increase in cell count in calculated by one-way ANOVA compared with media control $(0 \mathrm{ng})$ * $\mathrm{P}<0.05,{ }^{* *} \mathrm{p}<0.001(\mathrm{n}=6)$. A control of PDGF (10 ng) was included.

\subsection{Discussion}

Previously, the bioactive MO-CM sample had been separated by SDS -PAGE, however no clear bands could be distinguished between any of the CM samples and the FBS in the media. Steps were taken to reduce the FBS in the sample and the OFM was labelled with FITC before the CM was collected in order to distinguish OFM derived (FITC labelled) 
and macrophage derived peptides. $\mathrm{OFM}_{\mathrm{F}}$ and OFM CM controls without macrophages were included to show any "leeching" of FITC from the samples.

Macrophages are able to survive for up to 48 hours in serum free media. The first step to separation was to repeat the collection of CM using only $1 \%$ FBS in the pre-conditioning of the OFM and none in the experiment. The macrophages that were cultured on $\mathrm{OFM}_{\mathrm{F}}$ showed the same uptake of this stain as they had previously done with Congo red (Figure 5-1). The fluorescent label shows clusters of fluorescence within the cells, indicating that the fluorescent label is being taken up into phagocytotic vesicle.

\subsubsection{A distinct $12 \mathrm{kDa}$ OFM derived peptide may be cleaved by macrophages}

Unlike the CMs in Chapter Four (Figure 4-6), clear bands could be distinguished using this low serum method (Figure 5-2) and by staining the OFM with covalently bound fluorescein to exposed free amine side chains of the matrix. Unlike Congo red, fluorescein will stay attached to any liberated smaller proteins released by the ECM whose size could then be determined by SDS-PAGE. The majority of the proteins in the CMs stained only with Coomassie; indicating that they were derived from the macrophages. Figure 5-2 demonstrated a protein band generated in the $\mathrm{MO}_{\mathrm{F}}-\mathrm{CM}$ between 10 and $15 \mathrm{kDa}$ in size which was fluorescently labelled.

Smaller bands $(<10 \mathrm{kDa}$ ) are present in lanes 1 and 4 (all samples where OFM was stained with FITC). As these bands are also present in the sample of labelled OFM that was not cultured with any cells, this small band is likely to be the unbound FITC that has leached from the OFM during the incubation. A large molecular weight band (around 100kDa) is observed in all lanes; this may be auto fluorescent serum protein bound to the OFM during the pre-conditioning of the material for cell seeding. Alternatively, larger $\mathrm{OFM}_{\mathrm{F}}$ peptides may come into solution during this incubation without cell-mediated proteolysis. 


\subsubsection{Enrichment of peptide A by DEAE anion exchange}

DEAE separation was used to enrich the fluorescent sample from the macrophage-derived peptides. As shown in Figure 5-6 and 5-7, both batch separation and gel filtration with DEAE in a gradient of tris buffer at $\mathrm{pH} 8.3$ led to enrichment of the new peptide.

The labelling of the ECM protein with FITC may inhibit the activity of the peptide if fluorescein attached to the amine side chains of the peptide interferes with ligand-receptor binding. This could also prevent the cleavage of proteins by trypsin during protein identification. For this reason, the fluorescently labelled ECM molecules were used as a trace in the separation of the peptide of interest from the unlabelled MO-CM sample. This meant an unlabelled protein was separated in parallel from the columns in the same fraction as the fluorescently labelled fraction. This single protein could then be used for identification and bioactivity, as this unlabelled protein is more likely to possess the native structure of the OFM and retain bioactivity. The assumption was made that during this separation, the FITC labelling of the protein did not change the ionic strength required to remove peptides from the DEAE beads. Figure 5-6 shows that the protein peak eluted from the DEAE using labelled (A) and unlabelled (B) proteins were collected in fractions 7 to 11 .

\subsubsection{Bioactivity of peptide A in MSC migration assay}

The original sample of macrophage OFM culture CM (MO-CM) showed a significantly higher level of chemotaxis for MSCs in relation to similar samples prepared from M-CM and O-CM (Chapter Four). The samples collected in Chapter Four contained a high FBS concentration, yet the MO-CM combination still gave a chemotactic sample. The distinct band shown in this chapter is the only obvious peptide that is generated by the combination of culturing macrophages and OFM in the same dish. An enriched sample of this peptide shows a significant level of chemotaxis in relation to the media control (Figure 5-7). 


\subsubsection{Peptide A: a novel matricryptin?}

Evidence from the fluorescent profile of peptides from control samples suggests that this peptide is derived from OFM but requires cleavage by macrophages. There are a number of ways that they may achieve this. The lower level of serum might aid the action of proteases expressed by macrophages that breakdown the OFM, in the same way that macrophages breakdown ECM in living tissue. What is peculiar is the fact that, unlike pepsin digestion, there is only one distinct band in the sample. It is plausible that a very specific cleavage of the OFM is taking place in this culture.

Macrophages growing on OFM (MO-CM) cause the liberation of peptides and give a bioactive sample. Interestingly, the action of macrophage CM (M-CM) on powdered OFM does not give the same activity. Macrophage CM (M-CM) was removed from the culture and incubated with powdered OFM. The resulting sample (in theory a digestion of OFM by expressed macrophage proteases) did not display significant bioactivity (data not shown). This may be because the proximity of macrophages to the OFM is necessary to release this specific peptide. If the macrophage proteases responsible for this activity are found on the cell surface (for example by an MT-MMP), cleavage of proteins may occur as macrophages move across the OFM surface. Alternatively, the macrophages may need to be constantly expressing proteases to yield a concentration of enzymes high enough to induce ECM breakdown concentration and counteract the inhibition or breakdown of active proteases.

In a study by Werb et al., an image of a macrophage on the surface of a collagen scaffold material shows that the surface of the material around the macrophage is reduced to a smooth layer, reproduced in Figure $5-8^{203}$. They demonstrated that macrophages on a collagen substratum were capable of modifying the surface properties of their substrate. To quote directly: "Scanning electron micrograph of macrophages cultured on complete R22 SMC matrix in serum-free medium in the presence of $10 \mathrm{p} . \mathrm{g} \mathrm{PIg} / \mathrm{ml}$ for $72 \mathrm{~h}$. Note the zone of clearing around the macrophage (MAC) that contains only a few amorphous elastin particles (e). Collagen fibrils (c) on the surface of the dish are barely visible at this 
magnification. At a distance from the cell the matrix has a friable appearance (arrow), as compared with a control matrix (inset at upper left), which was incubated without cells."

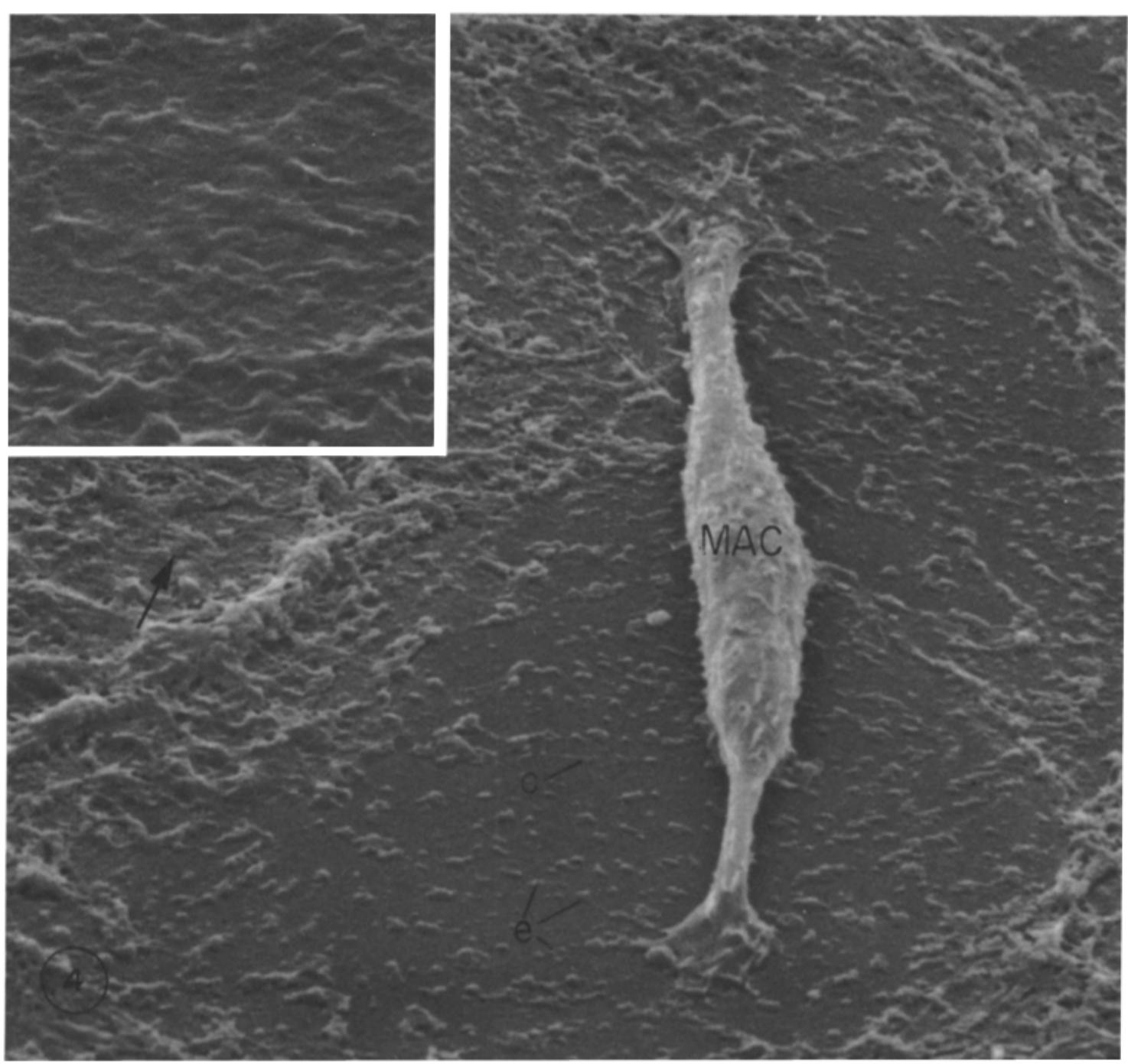

\section{Figure 5-8 SEM of macrophage on ECM}

The macrophage cleaves away contours of a collagen surface; taken from Werb et al. $1980^{203}$.

\subsubsection{Limitations}

While the prospect of a novel matricryptin is exciting, there are several limitations of this method to consider. Firstly, the fluorescent peptide was enriched, not isolated from the MO-CM rendering the bioactive peptide A impure. Secondly, due to limited amount of 
protein produced using this method, a full analysis of its activity using different cells and models is difficult. 


\section{Identification of peptides by mass}

\section{spectrometry}

\subsection{Summary}

Mass spectrometry (MS) was used to analyse the macrophage OFM CM sample (MO-CM) that was found to be bioactive in the MSC chemotaxis assay. MALDI TOF/TOF MS (matrix-assisted laser desorption/ionisation - time of flight) and LC ESI MS/MS (liquid chromatography coupled electrospray ionisation) methods were used to identify peptides within the MO-CM samples. Repeated experiments using MALDI failed to render any protein matches for this sample, however ESI analysis showed a number of high scoring proteins from the Ovis aries protein database including decorin (DCN), keratin and actin.

\subsection{Introduction}

The biological sample of interest appeared on one-dimensional (1D) SDS-PAGE as two distinct bands; one more than $75 \mathrm{kDa}$ and another between 10 and $15 \mathrm{kDa}$ (Figure 4-2). During the attempt to separate the fluorescent band from the rest, it became apparent that several proteins of a similar size were present within the sample. It is possible that these are derived from macrophage-CM and other fragments of OFM generated by proteolytic digestion of the biomaterial by macrophages. In the previous chapter, several proteases were suggested as candidates for this process including MMP-1, MMP-3, MMP-7, MMP-9 and MMP-12. In this chapter, undiluted samples and partially purified samples were identified by MS. Once identified, these fragments can be used in a number of ways in more complex animal models to establish whether they promote wound healing.

MALDI and ESI MS techniques have been used to identify biomolecules to great effect since their inception. These methods allow for the mass of a peptide to be measured based 
on its charge state (z) and its mass to charge ratio (m/z). MS analysis of a sample requires the ionization of peptides into the gas phase that can be differentiated and detected based on the mass to charge ratio (m/z). Traditionally, MS methods required samples to be vaporised and ionized in a manner that is overly destructive to heat labile biological peptides, however "soft ionization" methods such as MALDI and ESI are available ${ }^{167}$.

MALDI can be used to identify peptides in heterogeneous mixtures, however complex samples can lead to reduced sensitivity because additional compounds generate a competing signal ${ }^{204}$. Another problem with mixed samples is known as "ion suppression" where one molecule inhibits the ionisation of another. Consequently, the biological sample is often prepared by removing a "band" or "spot" from gel bands separated by $1 \mathrm{D}$ or 2D electrophoresis to reduce complexity. The proteins in the sample are embedded on the matrix that absorbs irradiation, volatilising and ionizing the sample, then the mass to charge ratio $(\mathrm{m} / \mathrm{z})$ can be detected. Given that MALDI generates a signal of charge $(+1)$ per peptide, the calculation of mass is straightforward: $m=m / z$, where $z=1$. Because each $m / z$ recorded represents one peptide, this method can be used to distinguish heterogeneous samples more simply than other methods. The disadvantage of the single charge state is that $\mathrm{m} / \mathrm{z}$ must be in the range detected by the instrument. Also these ions do not fragment as readily, making tandem MS more difficult.

ESI can be coupled with liquid chromatography to separate peptides before they are sprayed in a fine mist over a high current to achieve ionization ${ }^{204}$. Unlike MALDI, a range of charged species is generated giving $+2,+3$ and +4 charged peptides. ESI MS generates a number of peaks per peptide, making the analysis more complex than MALDI. On the other hand, since a range of $\mathrm{m} / \mathrm{z}$ values can be detected for each species, each peptide is more likely to be detected within the $\mathrm{m} / \mathrm{z}$ range of the instrument. ESI is less tolerant to contamination by salts than MALDI and steps must be taken to remove residual salts from a sample. 


\subsection{Aims}

The sample separated by SDS-PAGE seen in Chapter Five, appeared to be predominantly one or two bands that were excised from the 1D SDS-PAGE gel. MALDI TOF MS was used to attempt to identify peptides within the MO-CM sample, a mixture of macrophage and OFM derived proteins. Further analysis of the undiluted dried sample and gel cut sample was carried out with ESI MS. Peptide matches that may have been derived from OFM material or the macrophage cells were identified. The likelihood of these being responsible for the bioactivity seen in Chapter Five will be discussed.

\subsection{Results}

\subsubsection{MALDI analysis of proteins in MO-CM sample}

A piece of the SDS-PAGE gel shown in Figure 6-1 was removed and processed for MALDI TOF MS analysis. The sample corresponded to the position of the fluorescently labelled band of interest in the box in lane one, but also contained other proteins (as shown in the Coomassie stained gel). In the gel below, the dotted box is the gel piece that was removed for analysis.

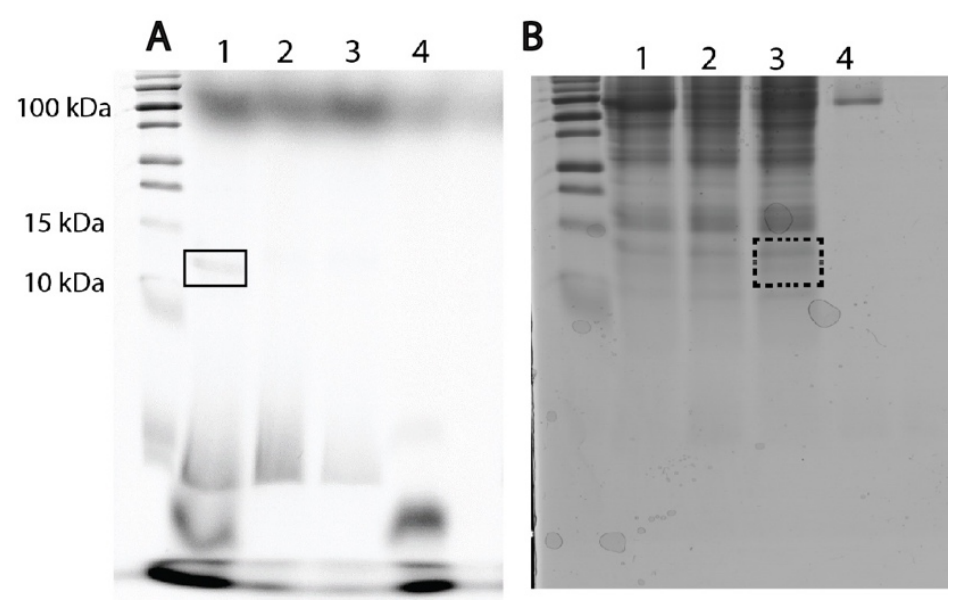

Figure 6-1 Gel extraction of proteins in the MO-CM sample

Samples of MO-CM were run on SDS-PAGE and scanned with a FITC filter (A) to 
show OFM-derived protein (inside box). The stained gel (B) shows the total protein in the sample (B). Proteins were extracted from the dotted box of B lane 3. These proteins correspond to the fluorescently labelled band in the sample of $\mathrm{MO}_{\mathrm{F}} \mathrm{CM}$ (A lane 1).

\subsubsection{MALDI TOF/TOF MS analysis of gel band}

The gel piece (dotted box in Figure 6-1) containing proteins around the same size as the fluorescently labelled fragment, were subjected to MALDI TOF MS analysis. This sample will be referred to as MO-CM 1D (MO-CM separated by 1D SDS-PAGE). A search of the MALDI TOF MS spectra gave a number of matches (Table 6-1). All of these protein matches are derived from mouse proteins (Mus muscularis). A search for protein matches in the Ovis aries database did not yield any matches.

\begin{tabular}{lll} 
PROTEIN ID & NAME & SPECIES \\
\hline GI 26389590 & UNAMED 15 KDA PROTEIN & MUS MUSCULARIS \\
GI 6754696 & MACROPHAGE INHIBITORY FACTOR 12KDA & MUS MUSCULARIS \\
GI 47606335 & UNAMED HEAT SHOCK PROTEIN 10KDA & MUS MUSCULARIS
\end{tabular}

\section{Table 6-1 MASCOT results for proteins identified by MALDI TOF MS}

Assuming that the fluorescently labelled sample is derived from OFM, it is possible that the presence of macrophage-derived proteins in the gel band are preventing the identification of this protein. Further enrichment of this protein carried out in Chapter Five showed that the labelled band was not visible by Coomassie staining, as previously discussed.

Using gel excised samples of the band of interest, after enrichment by DEAE described in the previous chapter, the significant loss of yield generated spectra indistinguishable from control samples of a gel piece excised from an area that did not contain any protein. The peaks around 800 to 880 in this spectra were also seen in samples of an excised gel piece without protein and are likely to be the breakdown products of Coomassie and acrylamide. 

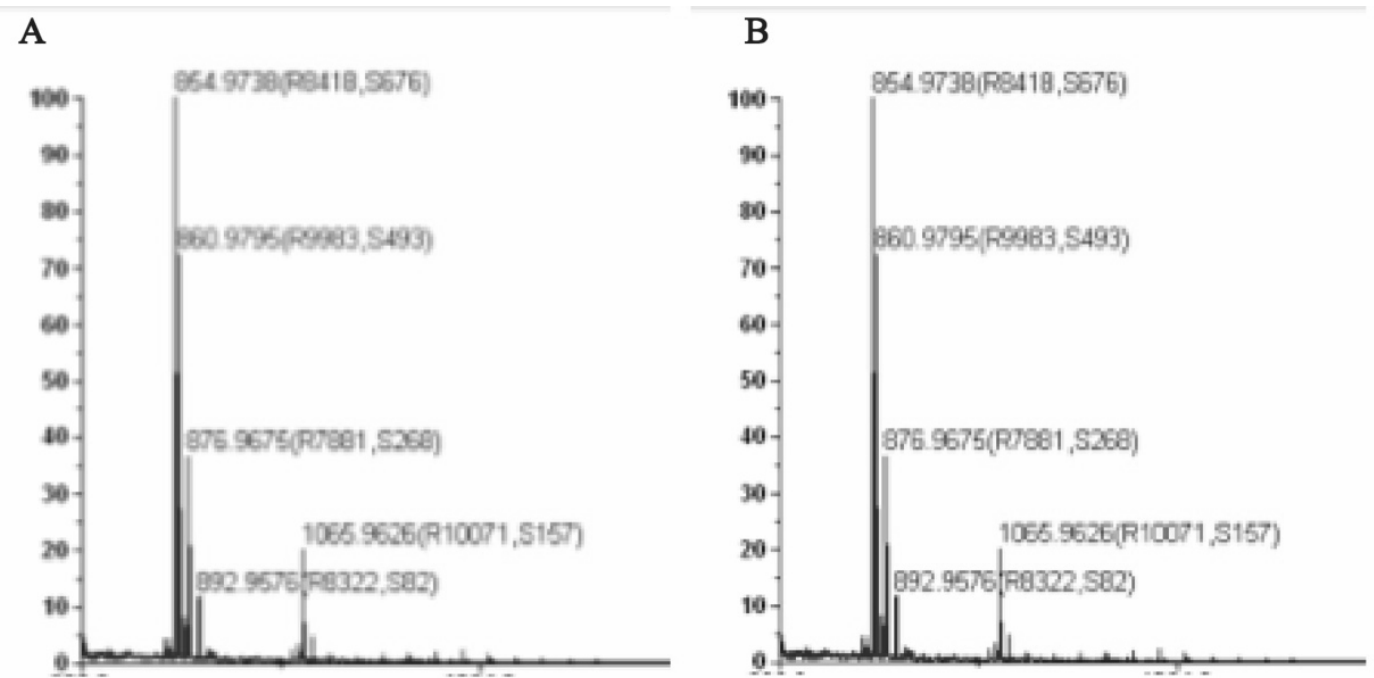

Figure 6-2 MALDI TOF MS spectra of enriched fraction of MO-CM 1D

Spectra derived from gel extracted samples containing enriched protein of interest (A) and gel pieces without protein (B). No unique peptides are observed in the MO-CM sample.

The MASCOT (Matrix Science, UK) search of these peaks did not render any peptide matches. A number of samples were used for the identification of the fluorescent target protein, the preparation of these samples is described in Chapter Five, however none of these generated a spectrum with distinct peaks that could be identified by MASCOT.

The concentrated samples of MO-CM and MO-CM 1D, were also sent to the Australian Proteome Analysis Facility (APAF) for MALDI TOF analysis to confirm this result. The MS spectrum of the gel-excised sample (MO-CM 1D) is shown in Figure 6-3. The APAF analysis was unable to discover any protein matches in MASCOT from this spectra (Appendix 4). 


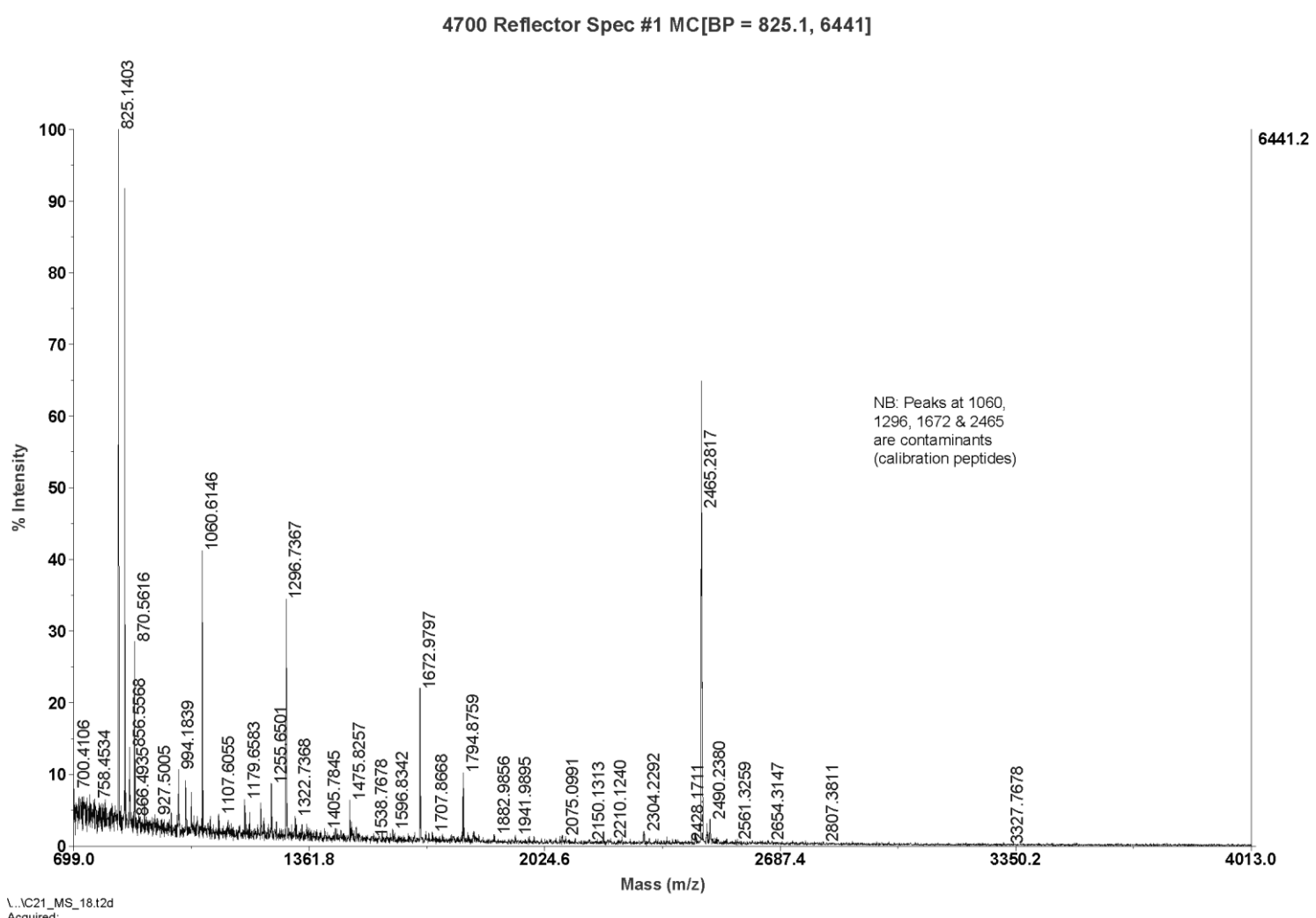

Figure 6-3 MS spectrum of peptides generated by MALDI TOF/TOF MS by APAF

MS spectra generated by a third party did not appear to generate novel peptides. A large number of peptides with a $\mathrm{m} / \mathrm{z}$ around 800 are seen in this spectra

As previously discussed, MALDI TOF MS is an effective way to identify proteins in a biological sample, however the way this sample was prepared did not give any matches from the ovine database. Despite multiple attempts, analysis by MALDI on purified and enriched samples, did not provide any significant protein matches. A likely reason for this is the sample complexity, which may have been underestimated because of the apparent "single band" in the SDS-PAGE gels in Chapter Five. It is possible that a more meticulous separation of proteins, such as by 2D gel spot excision, may yield better results.

Although MALDI TOF MS has been indicated as a method that is capable of identifying peptides in heterogeneous samples, the number of proteins in the sample was never truly quantified. A possible cause of distorted results in a mixed sample are "ion suppression" when the molecule is inhibited by others, or a loss of sensitivity because the signal from the molecule of interest is being dwarfed by other peptides. In some cases, peptide identification by ESI is a favourable approach. 


\subsubsection{LC ESI MS/MS analysis of dried sample and gel band}

The same samples, MO-CM undiluted and MO-CM 1D, were analysed by ESI MS at the Australian Proteome Analysis Facility (APAF). These samples were used as they were freshly prepared with the highest protein concentration. For this analysis, both the undiluted sample (dried) and a gel piece were used. A number of protein matches were found using ESI MS (Table 6-2 and 6-3). Protein matches are shown for the three highest scoring proteins. 


\begin{tabular}{|c|c|c|c|c|}
\hline Tab & PROTEIN & $\begin{array}{l}\text { SIZE } \\
\text { (DA) }\end{array}$ & SCORE* & $\begin{array}{l}\text { PEPTIDE OBSERVED MASS } \\
\text { AND SEQUENCE }\end{array}$ \\
\hline 10 & Q9TTE2 & 39,947 & 1295 & 550.3283 K.ISPGAFAPLVK.L \\
\hline 6-2 & DECORIN & & & 389.2077 K.ITEIKDGDFK.N \\
\hline & & & & 431.5934 K.NLHTLILINNK.I \\
\hline MA & & & & 833.9335 K.DLPPDTALLDLQNNK.I \\
\hline $\mathrm{SC}$ & & & & 664.3621 K.VPKDLPPDTALLDLQNNK.I \\
\hline & P60713 & 41,710 & 797 & 398.2388 K.IIAPPER.K \\
\hline OT & ACTIN, CYTOPLASMIC & & & 566.7669 R.GYSFTTTAER.E \\
\hline nrt & & & & 581.3102 K.EITALAPSTMK.I \\
\hline pive & & & & 400.2392 R.AVFPSIVGRPR.H \\
\hline ein & & & & 506.2394 K.QEYDESGPSIVHR.K \\
\hline & & & & 597.6354 K.SYELPDGQVITIGNER.F \\
\hline mat & & & & 895.9552 K.SYELPDGQVITIGNER.F \\
\hline che & & & & 652.0258 R.VAPEEHPVLLTEAPLNPK.A \\
\hline & Q9MZA9 & 17,161 & 524 & 435.7210 R.FANYIDK.V \\
\hline$s$ of & VIMENTIN (FRAGMENT) & & & 453.7364 R.FLEQQNK.I \\
\hline und & & & & 558.2944 K.VELQELNDR.F \\
\hline und & & & & 375.8723 R.FANYIDKVR.F \\
\hline ilut & & & & 585.3605 K.ILLAELEQLK.G \\
\hline & & & & 770.4561 K.ILLAELEQLKGQGK.S \\
\hline ed & & & & 529.9372 R.TNEKVELQELNDR.F \\
\hline MO & O77727 KERATIN & 48,740 & 420 & \\
\hline - & O46390 BIGLYCAN & 41,497 & 172 & \\
\hline CM & Q6B7M7 COFILIN-1 & 18,507 & 109 & \\
\hline fro & Q6YNC8 HISTONE & 13,545 & 66 & \\
\hline $\mathrm{m}$ & P62896 CYTOCHROME C & 11,696 & 50 & \\
\hline Swi & Q9BGM5 KERATIN & 49,282 & 46 & \\
\hline ssPr & Q28554 GLYE-3-PDEHYDROGENASE & 34,710 & 44 & \\
\hline ot, & P15241 KERATIN, TYPE II & 53,647 & 40 & \\
\hline Ovi & P21814 UTERINE MILK PROTEIN & 49,191 & 30 & \\
\hline $\boldsymbol{s}$ & Q83957 NUCLEOPROTEIN & 43,394 & 28 & \\
\hline arie & (*) SCORE > 19 IS SIGNIFICANT & & & \\
\hline
\end{tabular}

$S$. 
In the table above, a large number of proteins are matched from the $\mathrm{CM}$ sample, including decorin, actin and vimentin. Using the gel excised samples, a smaller number of matches were established, including actin, keratin and decorin (Table 6-3).

\begin{tabular}{|c|c|c|c|}
\hline PROTEIN & $\begin{array}{l}\text { SIZE } \\
\text { (DA) }\end{array}$ & SCORE* & $\begin{array}{l}\text { OBSERVED MASS AND PEPTIDE } \\
\text { SEQUENCE }\end{array}$ \\
\hline \multirow{4}{*}{$\begin{array}{l}\text { P60713 ACTIN, } \\
\text { CYTOPLASMIC } 1\end{array}$} & \multirow[t]{4}{*}{41,710} & \multirow[t]{4}{*}{196} & 398.2400 K.IIAPPER.K \\
\hline & & & 566.7674 R.GYSFTTTAER.E \\
\hline & & & 581.3128 K.EITALAPSTMK.I \\
\hline & & & 589.3095 U K.EITALAPSTMK.I + OXIDATION (M) \\
\hline \multirow{2}{*}{$\begin{array}{l}\text { O77727 KERATIN, TYPE I } \\
\text { CYTOSKELETAL } 15\end{array}$} & \multirow[t]{2}{*}{48,740} & \multirow[t]{2}{*}{174} & 404.2031 R.LAADDFR.L \\
\hline & & & 516.2998 R.VLDELTLTK.T \\
\hline \multirow[t]{2}{*}{ Q9TTE2 DECORIN } & \multirow[t]{2}{*}{39,947} & \multirow[t]{2}{*}{84} & 550.3297 K.ISPGAFAPLVK.L \\
\hline & & & 550.3300 K.ISPGAFAPLVK.L \\
\hline \multirow{2}{*}{$\begin{array}{l}\text { Q9BGM5 KERATIN, TYPE I } \\
\text { CYTOSKELETAL } 25\end{array}$} & \multirow[t]{2}{*}{49,282} & \multirow[t]{2}{*}{54} & 516.3036 R.VLDVITLCR.T \\
\hline & & & 545.7676 K.VTMQNLNDR.L \\
\hline
\end{tabular}

(*) SCORE > 11 IS SIGNIFICANT

\section{Table 6-3 MASCOT protein matches of gel excised MO-CM from SwisProt, Ovis aries}

A large number of protein matches were identified in the dried sample and the gel band sample, as indicated above. All the proteins in this table score above the threshold, indicating that it is likely these proteins are found in the sample. The highest scoring protein in the undiluted sample is the decorin (DCN) protein (Table 6-2), which is also present in the gel excised sample (Table 6-3).

The samples of particular interest are the ones that are found in the gel band, although the additional steps to extract the gel band may have led to a reduction in the amount of protein and thus the likelihood of identification. The list includes actin, keratin and decorin.

It is possible that all the proteins in the Table 6-2 are present in the CM sample, however their abundance and possible bioactivity is not known. These proteins could be derived from the OFM biomaterial or added to the sample during the manipulation of the material or as an artefact of the culture the sample was collected from. 
The origins of these proteins can be rationalised to a degree. Firstly, keratin is often found in samples that have been handled extensively. There are many stages in the process of collecting this sample that keratin may have been introduced; during the animal slaughter, decellularisation of OFM, packing and sterilisation, or during the culture set up. It is also known that there is little keratin in the rumen matrix, so it is less likely that this protein is a major constituent of OFM.

Actin and vimentin are very ubiquitous cellular proteins; these structural proteins are present in almost all mammalian cells. It is possible that these are left over from the decellularisation process of OFM or expressed by the macrophage in the CM.

Decorin is a proteoglycan that is known to associate with collagen I. It is possible that $\mathrm{DCN}$ is being cleaved from collagen I by the macrophages. It is interesting to note that peptides from the entire protein are observed in the undiluted dried sample, but only two small peptides are generated from gel excised from size-excluded fractions of the sample. As DCN is $39 \mathrm{kDa}$ in size and the protein of interest appears on SDS-PAGE as a band between 10 and $15 \mathrm{kDa}$, it possible that derivatives of the DCN protein are being released by the action of macrophages or macrophage secreted proteases. The peptides identified from both samples do not cover the whole decorin protein.

\subsubsection{Size exclusion chromatography with Superdex 75}

The undiluted MO-CM sample was fractionated according to size with a Superdex 75 column by APAF. Figure 6-4 shows the chromatogram of the sample and the standards. A distinct peak is seen in the earlier fractions (greater than $75 \mathrm{kDa}$ ) these were collected and pooled as sample A1. A smaller group of peaks was similarly collected and pooled as A2. While the larger sample appears as a single peak, the smaller one does not have a distinct peak and may be a heterogeneous mixture of proteins in the range of 13 to $58 \mathrm{kDa}$. 
A

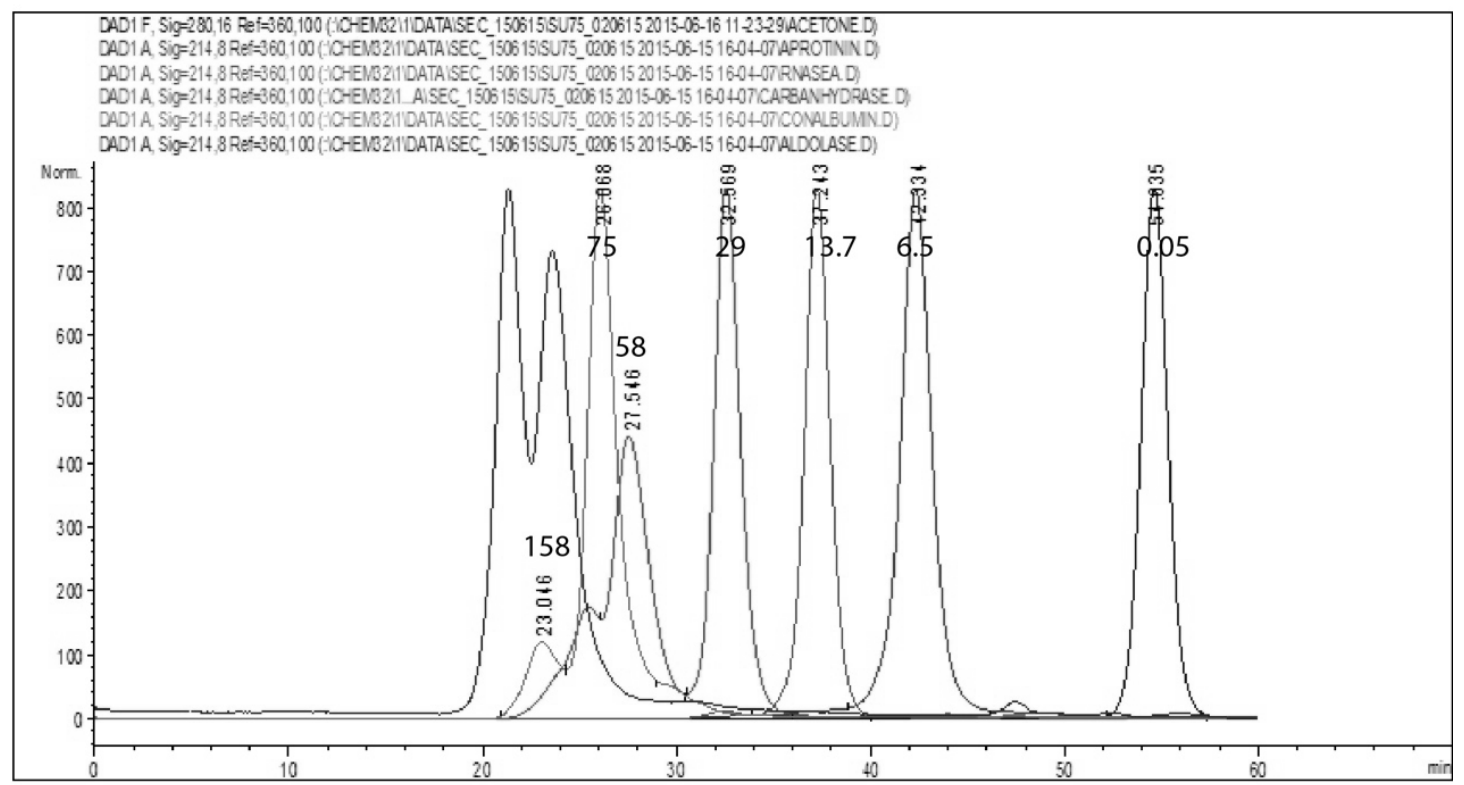

B

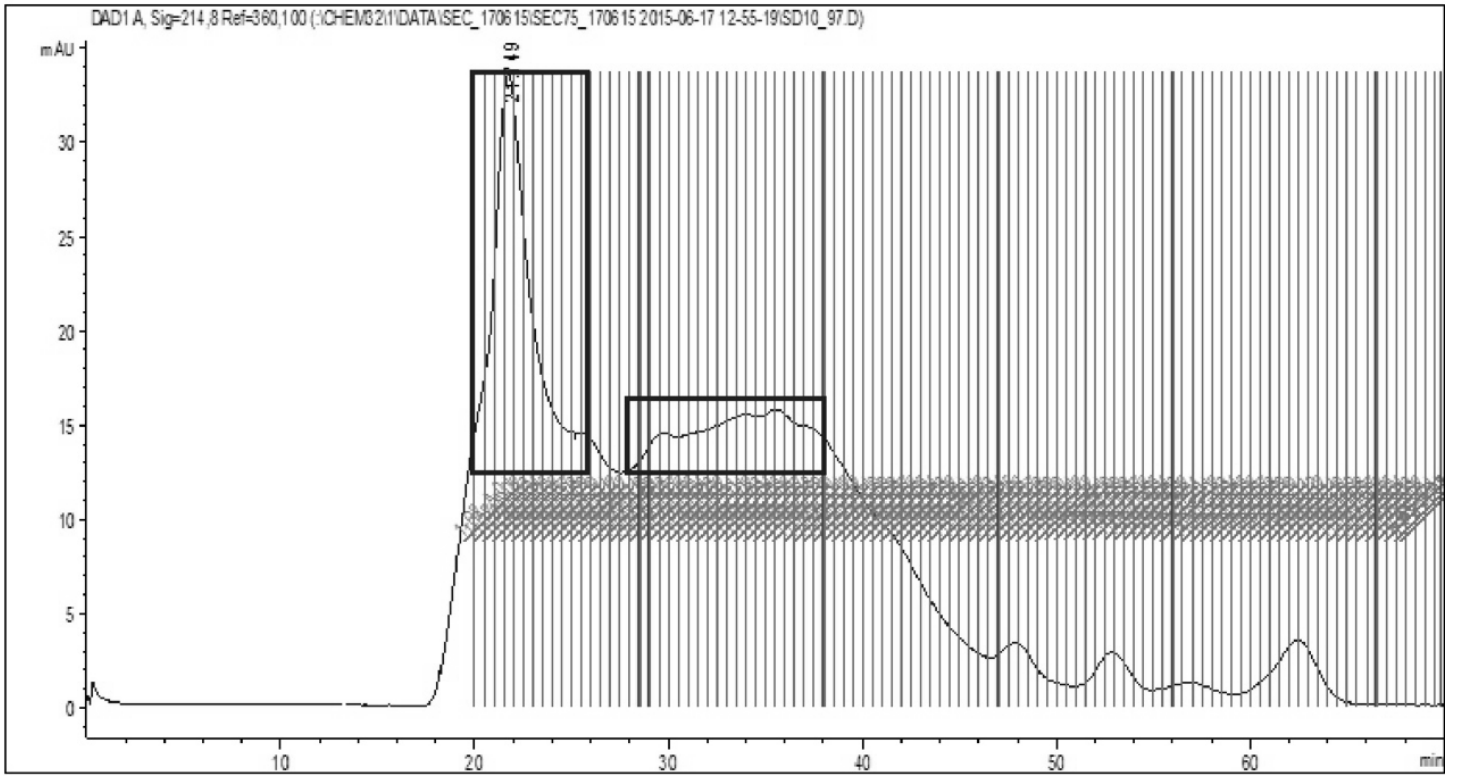

Figure 6-4 Size exclusion chromatogram MO-CM sample

In (A), the standards show the retention time (vertical) and the mass (horizontal) of each standard. These are used to calculate the size of peptides with peaks in the sample (B). A1 $(>75 \mathrm{kDa})$ was collected from fractions with a retention time of $20-26$ minutes and A2 $(13-58 \mathrm{kDa})$ was collected from fractions within the retention time of 28 and 38 minutes.

The samples collected above were analysed using ESI MS. Peptides masses were searched for using both mouse (Mus musculus) and sheep (Ovis aries) databases. Table 6-4 lists the protein matches from samples $\mathrm{A} 1(>75 \mathrm{kDa})$ and $\mathrm{A} 2(13-58 \mathrm{kDa})$. 
PROTEIN SIZE S PEPTIDE

(DA)

\begin{tabular}{|c|c|c|c|c|}
\hline \multirow[t]{4}{*}{$\begin{array}{l}\text { PEAK A1 } \\
(>75 \mathrm{KDA})\end{array}$} & KERATIN TYPE I & 49282 & 65 & $\begin{array}{l}\text { 355.5414 R.LASYLENVR.A } \\
555.2561 \text { R.DAEAWFNEK.S }\end{array}$ \\
\hline & DECORIN OS & 39947 & 42 & 624.3236 R.VVQCSDLGLEK.V + CARBAMIDOMETHYL (C) \\
\hline & GLYCERALDEHY & 34710 & 25 & 685.3836 R.GAAQNIIPASTGAAK.A \\
\hline & ACTIN & 41710 & 24 & 589.3136 K.EITALAPSTMK.I + OXIDATION (M) \\
\hline \multirow{3}{*}{$\begin{array}{l}\text { PEAK A2 } \\
(13.7-58 \\
\text { KDA })\end{array}$} & $\begin{array}{l}\text { KERATIN, TYPE I } \\
\text { CUTICULAR }\end{array}$ & 50455 & 34 & 404.2059 K.LAADDFR.T \\
\hline & DECORIN OS & 39947 & 24 & 624.3181 R.VVQCSDLGLEK.V + CARBAMIDOMETHYL (C) \\
\hline & $\begin{array}{l}\text { PAZ DOMAIN } \\
\text { CONTAINING }\end{array}$ & 179978 & 18 & $\begin{array}{l}552.8301 \text { K.LLYQVQASKQEITAVLEMK.S + OXIDATION } \\
\text { (M) }\end{array}$ \\
\hline
\end{tabular}

$S=$ SCORE (ALL SCORES DIPSPLAYED ARE SIGNIFICANT)

Table 6-4 MASCOT matches of LCMS MO-CM with Ovis aries database.

The same decorin peptide match appears in both fractions, along with peptides from keratin, glyceraldehyde and actin (peak A1) and keratin and "PAZ domain containing protein" in peak A2. Only one DCN peptide was matched after the sample had been through size exclusion.

\subsubsection{Peptide mapping of DCN}

Decorin was a consistent protein match using ESI MS analysis of various samples. The coverage of peptides over the decorin protein was mapped using different enzymes during sample preparation. Table 6-5 lists DCN peptides identified for each sample preparation. ESI analysis of the peptide of interest in the MO-CM undiluted sample using two enzymes, was carried out to achieve greater coverage of the decorin protein. The fragments are underlined in Table 6-5.

The peptides from the sample that had been purified by size exclusion and gel extraction are included for comparison. Interestingly, the non-purified samples contained peptides from the whole protein, when digested with trypsin. For purified samples, both matched peptides are between 50 and 150 amino acids in size. 


\begin{tabular}{|c|c|c|}
\hline & PEPTIDE & COVERAGE OF DCN \\
\hline $\begin{array}{l}\text { NON } \\
\text { PURIFIED } \\
\text { SAMPLE } \\
\text { (TRYPSIN) }\end{array}$ & $\begin{array}{l}\text { K.DLPPDTALLDLQNNK.I } \\
\text { K.ITEIKDGDFK.N } \\
\text { K.NLHTLILINNK.I } \\
\text { K.ISPGAFAPLVK.L } \\
\text { K.TLQELR.V } \\
\text { K.VDAASLK.G } \\
\text { K.LGLSFNSISAVDNGSLANTPHLR.E } \\
\text { K.VPGGLADHK.Y }\end{array}$ & $\begin{array}{l}\text { MKATIIFFLV AQVSWAGPFQQKGLFDFMLE } \\
\text { DEASGIGPEE RFHEVPELEPMGPVCPFRCQ } \\
\text { CHLRVVQCSD LGLEKVPKDL PPDTALLDLQ } \\
\text { NNKITEIKDGDFKNLKNLHT LILINNKISK } \\
\text { ISPGAFAPLVKLERLYLSKNQLKELPEKMPK } \\
\text { TLQELRVHE NEITKVRKSV FNGLNQMIVV } \\
\text { ELGTNPLKS GIENGAFQGM KKLSYIRIAD } \\
\text { TNITTIPQGL PPSLTELHLD GNKITKVDAA } \\
\text { SLKGLNNLAK LGLSFNSISA VDNGSLANTP } \\
\text { HLRELHLNNN KLVKVPGGLA DHKY IQV } \\
\text { VYLHNNNISAIGS NDFCPPGYNT KKA } \\
\text { SYSGVSL FSNPVQYWEI QPSTFR CVYVRA } \\
\text { AVQLGNYK }\end{array}$ \\
\hline $\begin{array}{l}\text { NON } \\
\text { PURIFIED } \\
\text { SAMPLE } \\
\text { (GLUC) }\end{array}$ & $\begin{array}{l}\text { E.KVPKDLPPDTALLDLQNNKITE.I } \\
\text { E.RLYLSKNQLKELPE.K } \\
\text { E.LGTNPLKSSGIE.N }\end{array}$ & $\begin{array}{l}\text { MKATIIFFLV AQVSWAGPFQ QKGLFDFMLE } \\
\text { DEASGIGPEE RFHEVPELEP MGPVCPFRCQ } \\
\text { CHLRVVQCSD LGLEKVPKDL PPDTALLDLQ } \\
\text { NNKITEIKDG DFKNLKNLHT LILINNKISK ISP } \\
\text { GAFA PLV KLERLYLSKN QLKELPE KMPK } \\
\text { TLQELRVHE NEITKVRKSV FNGLNQMIVV } \\
\text { ELGTNPLKSS GIE NGAFQGMKKLSYIRIAD } \\
\text { TNITTIPQGL PPSLTELHLD GNKITKVDAA } \\
\text { SLK GLNNLAKLGLSFNSISA VDNGSLANTP } \\
\text { HLREL HLNNN KLVKVPGGLA DHKY IQVV } \\
\text { YLHNNNISAIGS NDFCPPGYNTKKAS YSGVS } \\
\text { LFSNPVQYWEIQPSTFRCVYVRAAVQ } \\
\text { LGNYK }\end{array}$ \\
\hline $\begin{array}{l}\text { SIZE } \\
\text { EXCLUSION } \\
\text { GEL } \\
\text { EXTRACTION }\end{array}$ & $\begin{array}{l}\text { R.VVQCSDLGLEK.V } \\
\text { K.ISPGAFAPLVK.L }\end{array}$ & $\begin{array}{l}\text { MKATIIFFLV AQVSWAGPFQ QKGLFDFMLE } \\
\text { DEASGIGPEE RFHEVPELEPMGPVCPFRCQ } \\
\text { CHLRVVQCSD LGLEKVPKDL PPDTALLDLQ } \\
\text { NNKITEIKDG DFKNLKNLHT LILINNKIS K } \\
\text { ISPGAFAPLV KLERLYLSKN QLKELPEKMP } \\
\text { KTLQELRVHE NEITKVRKSV FNGLNQMIVV } \\
\text { ELGTNPLKSS GIENGAFQGMKKLSYIRIAD } \\
\text { TNITTIPQGL PPSLTELHLD GNKITKVDAA } \\
\text { SLKGLNNLAKLGLSFNSISA VDNGSLANTP } \\
\text { HLRELHLNNN KLVKVPGGLA DHKYIQ VVY } \\
\text { LHNNNISAIGS NDFCPPGYNT KKASYSGVSL } \\
\text { FSNPVQYWEI QPSTFRCVYVRAAVQLGNYK }\end{array}$ \\
\hline
\end{tabular}

Table 6-5 Peptide matches of DCN protein using trypsin and GluC

The peptides identified by ESI MS from the dried sample were digested with trypsin and gluC, seven peptides were identified in the trypsin digested sample and three peptides were identified in the gluC digested sample (Table 6-5). These peptides were mapped onto the decorin protein, to show the coverage of the decorin protein in these samples. The peptides generated from samples that had been prepared by 1D SDS-PAGE and size exclusion chromatography (SEC) were also mapped on the decorin protein. The range of peptides in the non-purified samples are spread across the entire protein, while the purified samples show only one peptide each (Figure 6-5). 


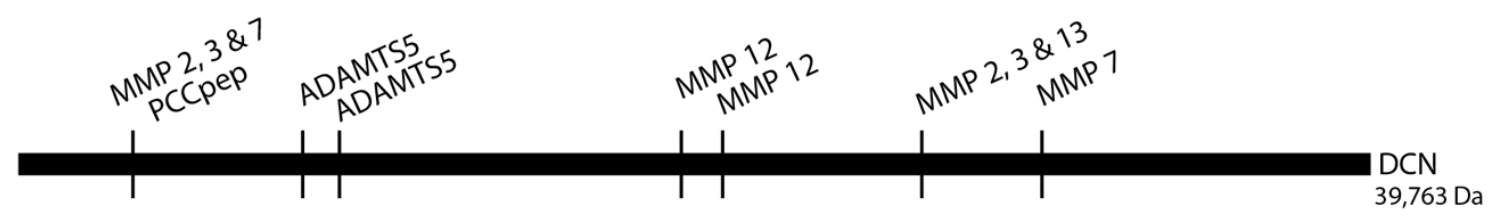

Trypsin

GluC

Trypsin Gel band

Trypsin SEC

Figure 6-5 The distribution of DCN derived peptides identified by ESI

Peptides generated from MO-CM, after digestion with trypsin and gluC are distributed across the whole protein. Single peptides identified from MO-CM after excision of a band from a 1D SDS-PAGE gel and SEC followed by trypsin digestion are both observed to the left of the MMP-12 cleavage sites.

\subsubsection{Theoretical digest of DCN by proteases}

The protein of interest appeared as a band in the SDS-PAGE between 10 and $15 \mathrm{kDa}$ (Chapter Five). The ESI data identified a matrix protein DCN that is $39 \mathrm{kDa}$ in size. A MEROPS database was used to explore this protein as a substrate for proteases. From this, the size of smaller peptides generated from the $39 \mathrm{kDa}$ DCN was calculated. Figure 6-6 A theoretical map of fragments generated by each protease is shown beneath the DCN protein, giving the size of fragments generated by each protease. 

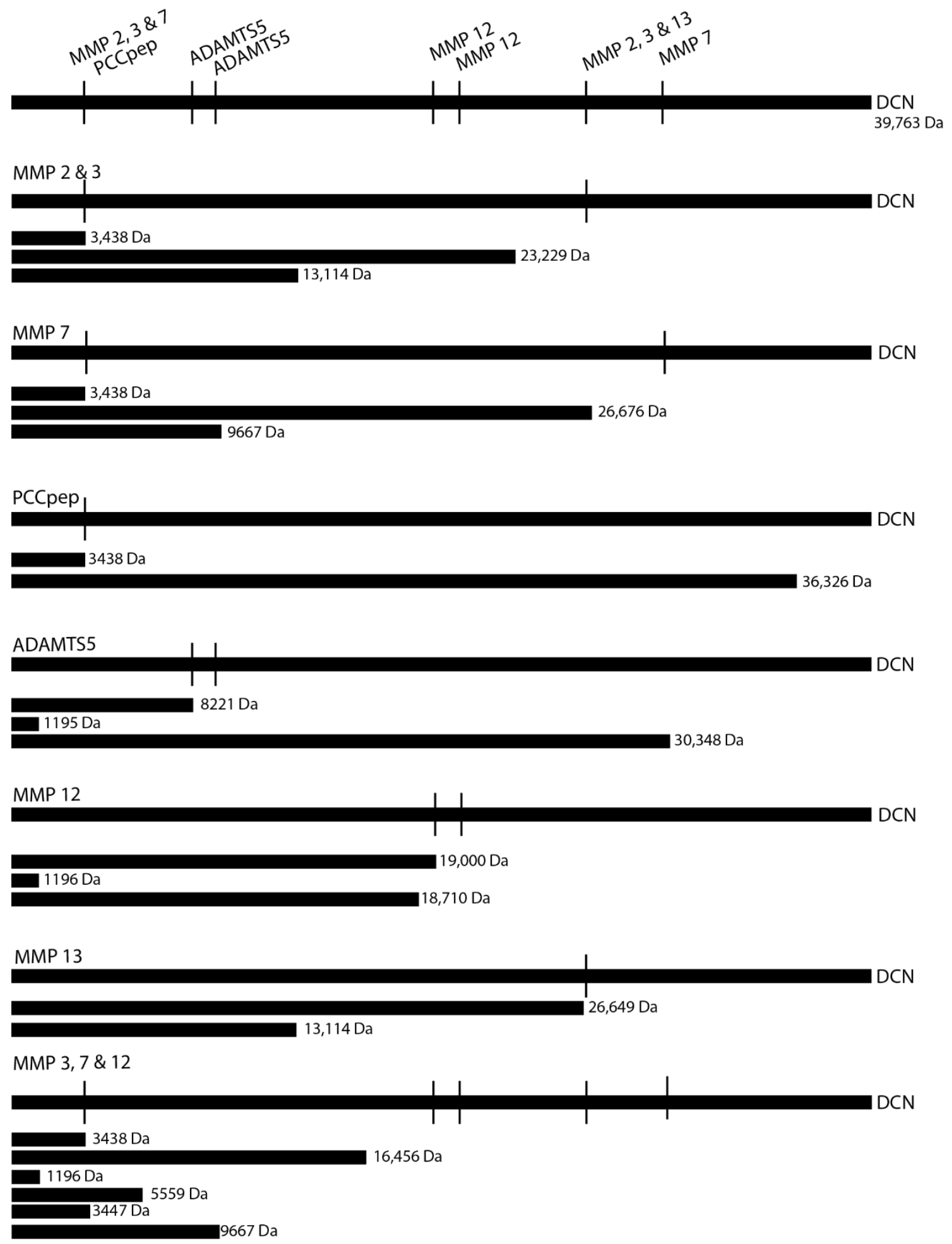

Figure 6-6 DCN fragments generated from known proteolytic cleavage sites

The top line shows the entire DCN protein with all known cleavage sites. Below this are fragments generated from each protease, along with their size (based on the amino acid sequence of each fragment). At the bottom the proteolytic cleavage of DCN using three 
proteases MMP-3, MMP-7 and MMP-12 is shown

\subsection{Discussion}

In this chapter, two ionisation methods were used to achieve the identification of samples by MS; MALDI and ESI. The CM sample generated by macrophage and OFM culture is likely to contain macrophage proteins, soluble OFM proteins and OFM proteins that are cleaved from the matrix directly by macrophages or indirectly by proteases expressed by the macrophages. A number of likely protein matches were identified as decorin fragments. This prompted a search for macrophage proteases that cleave decorin.

\subsubsection{Evidence for macrophage processing of DCN}

The role of macrophages in wound healing is outlined in Chapter One. It is important to note that there are distinct macrophage phenotypes assigned to subsets that influence the cell function during wound healing. Activation of macrophages from a resting state (Mo) can lead to two distinct phenotypes; classically activated macrophages (also called M1) or alternatively activated macrophages $(\mathrm{M} 2)^{140}$. In this M1/M2 model, M1 macrophages are stimulated to respond to infection and carry out an "inflammatory" function. Subsets of macrophages in this state are able to destroy foreign bacteria and recruit other immune cells to the location. Macrophages that display an M2 phenotype are linked to the rebuilding of the wound site, recruiting FBs and keratinocytes. The phenotypic switch involves changes in expression profiles, including chemokines and proteases that play an important role in wound closure.

There is some debate about the phenotype of wound healing macrophages across the different stages of wound healing. Sindrilaru et al. have suggested that the M1/M2 phenotypic switch between inflammatory and reconstructive macrophage phenotype is a simplification and that in fact macrophage subsets express different phenotypes during the four phases of wound healing ${ }^{147}$. During the early inflammatory phase, macrophages express an M1 phenotype that evolves to an M2 phase during the late inflammatory phase. 
During the granulation phase, macrophages express a "pro-fibrotic M2a" phenotype and during matrix deposition phase they express a "fibrolytic M2c phenotype". Roch et al. investigated MMP expression by comparing normal macrophage, "classically activated" (M1) macrophages and "alternatively activated (M2) macrophages ${ }^{205}$. Figure 6-7 illustrates their findings. Normal (or non-stimulated macrophages) are found to express MMP-7, MMP-8 and MMP-9; M1 macrophages express the same proteases as well as higher levels of MMP-1 and MMP-3 and MMP-10; M2 macrophages express MMP-7 and MMP-9 and higher levels of MMP-12 than the other subtypes.

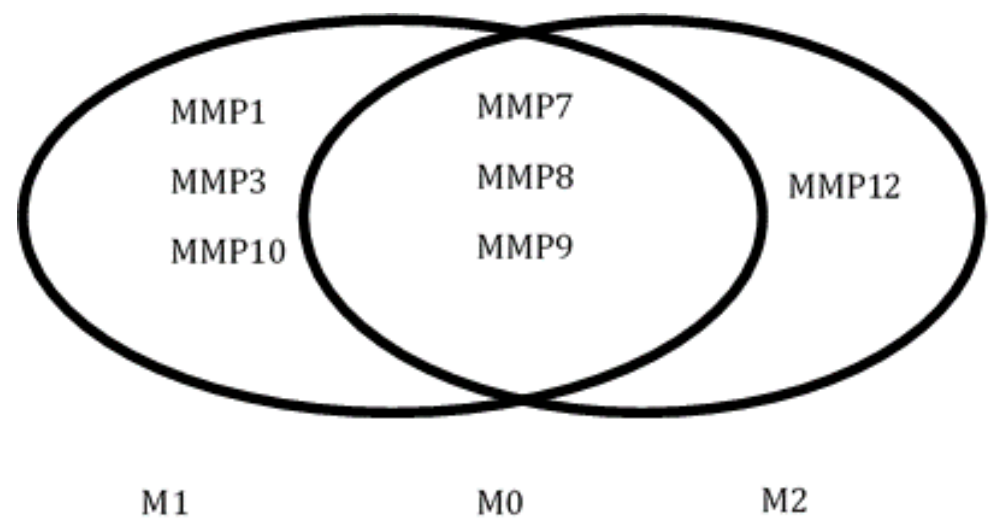

Figure 6-7 Phenotype associated MMP expression of macrophages

Data from Roch et al. show that subsets of macrophages express different MMPs depending on their activation. Non-activated macrophages (M0) express MMP-7, MMP-8 and MMP-9. Classically activated macrophages (M1) express the same MMPs, as well as MMP-1, MMP-3 and MMP-10. Alternatively activated macrophages express the same MMPs and Mo as well as MMP-12 205 .

Figure 6-7 shows MMPs expressed by M1 versus M2 macrophage subsets. MMP-1, MMP3 and MMP-10 are expressed by the M1 subset and MMP-12 alone is unique to M2 macrophages. Using the MEROPS database, DCN was found to be a substrate for a number of proteases, listed below:
I. MMP-2
II. MMP-3
III. MMP-7 
IV. MMP-12

V. MMP-13

VI. CCpep

VII. ADAM5Ts

A molecular weight calculator was used to give the size of fragments of DCN after cleavage by each MMP as shown in Figure 6-6. At the bottom of this figure, the theoretical digest of DCN by MMP-12, MMP-3 and MMP-7 is shown. These are all of the proteases expressed by M1 and M2 macrophages. It is not possible to say, whether the macrophages in the MO-CM culture were expressing an M1 or M2 phenotype. However, looking at all the possible DCN fragments generated from macrophage proteases, there are several smaller fragments of DCN present, including a $16 \mathrm{kDa}$ peptide. Both purified samples of the protein of interest lie within this $16 \mathrm{kDa}$ fragment of DCN (Figure 6-5).

\subsubsection{Evidence for DCN bioactivity}

The MS data points to a group of proteins or peptides that may be responsible for the bioactivity observed in Chapter Four, including the ECM protein decorin. The analysis of proteolytic cleavage sites within the protein, in conjunction with what is known about macrophage MMP expression, suggests that the peptide within the MMP-12 and MMP-2 /MMP-7 cleavage site is part of the peptide of interest that is derived from the OFM biomaterial. To establish how, why, or what fragments of DCN may be involved in MSC chemotaxis, it is important to look at its role in ECM stability and wound healing.

Decorin was characterised in 1986 by Krusius et $a l^{206}$. The researchers noted that the proteoglycan, also known as PGS2 and PG40, which associates with collagen, is expressed in many tissues and has a high pI of 9.8. Decorin expressed by Chinese hamster ovary cells was found to alter the phenotype of endothelial cells in culture, leading to greater spreading of the monolayer ${ }^{207,208}$. It has since been found that decorin binds to a number of ECM proteins including fibronectin, thrombospondin, collagens I, II, VI and XIV, as well 
as complement component $\mathrm{C} 1 \mathrm{q}$ and growth factor $\mathrm{TGFB}^{136,208-210}$. Collagen binding occurs primarily at LRR 4 and 5.

In terms of wound healing, decorin's most important roles are in collagen fibrogenesis and in its interaction with $\mathrm{TGF}^{211}$. Figure 6-8 shows the relationship between decorin and collagen, used with permission from Reed et al. ${ }^{212}$. The LRR in decorin forms beta strands which give the protein an "arched solenoid" shape with room to form attachments around individual collagen triple helices. It is thought that decorin bound to collagen in this way regulates lateral fusion of fibrils and maintains uniform interfibrilllar space. In Figure 6-8 (E), the collagen fibrils in a DCN deletion mutant appear non-uniform in both spacing and size. The result of a DCN deletion mutant on the organisation of collagen fibrils is shown in E compared with wild type DCN (D). DCN deletion mutant fibrils are not uniform in size and spacing.
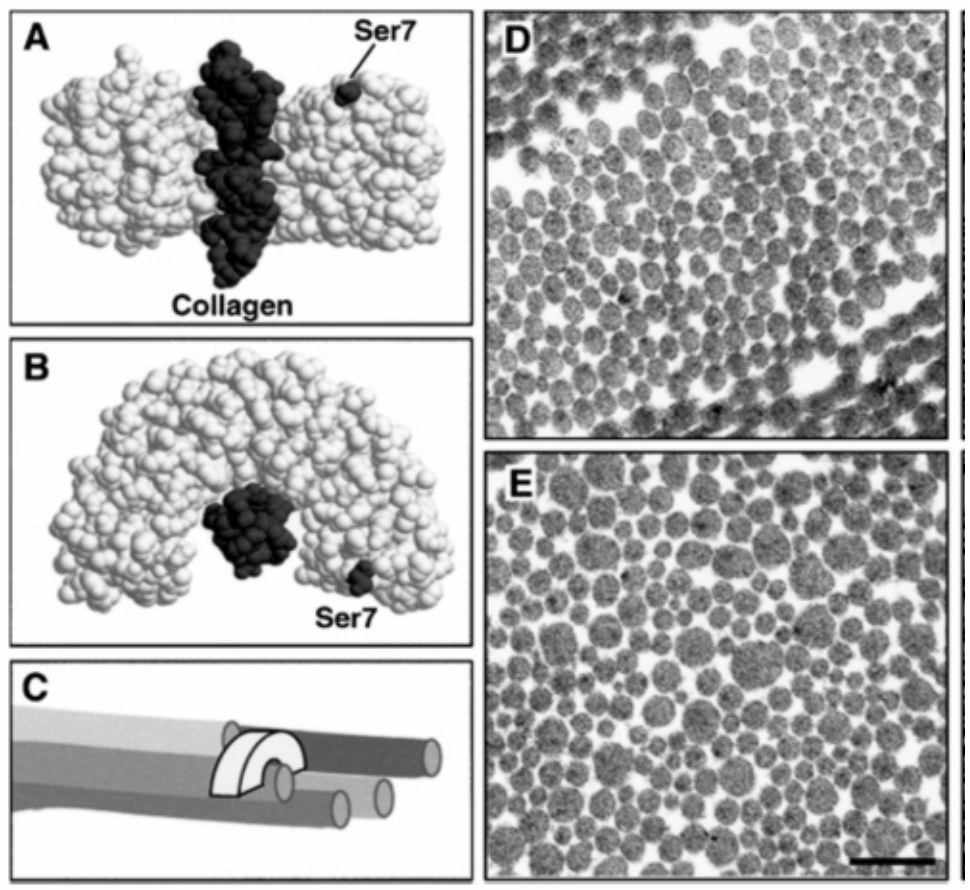

Figure 6-8 Decorin and collagen

The arched structure of decorin and its proposed interaction with a collagen triple helix is shown from beneath the collagen helix (A) and side on (B). This is represented 
schematically in $\mathrm{C}$, which shows the way decorin binds to collagen helix within fibrils. Image used with permission from Reed et al. ${ }^{212}$.

The other notable function of decorin in relation to wound healing is its ability to bind TGF $\beta$. In a study by Imai et al., decorin was incubated with MMP-2, MMP-3 and MMP-7 in solution ${ }^{213}$. DCN-TGF $\beta$ complexes incubated with these three enzymes led to the release of TGF $\beta$ into solution. MMP-7 had the greatest affinity for decorin digestion and led to peptides that were $9.5,15.5$ and $20 \mathrm{kDa}$ in size. In the theoretical digest of decorin in Figure 6-6, peptides with masses of 3.4, 9.6 and $26.6 \mathrm{kDa}$ were generated by known MMP-7 binding sites.

\subsubsection{Potential use for protein as a mediator of cell migration}

Evidence presented here suggests a possible pathway for the DCN fragment in a simple cell based chemotaxis assay. The potential for wound healing will need to be established in more complex mammalian models.

Another issue to be explored is the ease of purification on a larger scale, whether the protein can be generated as a recombinant protein and the cost of this relative to the extraction of the protein using the "cell-mediated" release of the fragment. It is possible that cellular processing of the fragment affects the bioactivity, in which case enzymatic digestion of DCN alone will not give the same result.

The concentration of growth factors during wound healing is dynamic, tissue specific and tightly controlled by cells. Chemokines such as PDGF and VEGF are not used as wound healing molecules because of the considerable cost of generating a recombinant protein in amounts required for wound healing, however, some trials have been carried out which use growth factors, either on their own or encapsulated in biomaterials, to alter the wound healing outcome in animal models $\mathrm{s}^{214,215}$.

It is possible that this protein can be used as a marker in biomaterials, and the augmentation of such a protein can be established by monitoring manufacturing processes to ensure the enrichment of this protein and its parent protein in materials. A purified 
version of the protein could be embedded into other biomaterials such as stents and meshes. 
Extracellular matrix derived peptides and mesenchymal stem cell motility Sandi Grainne Dempsey - September 2016 


\section{General discussion}

\subsection{Overview}

This work has uncovered a number of useful insights into the nature of the OFM biomaterial, the nature of protease released bioactives from OFM and interaction between the material and macrophage cells. In addition, a body of evidence is described that implicates macrophage-mediated cleavage of decorin protein from the biomaterial. The resulting cryptic peptides may be responsible for the MSC bioactivity generated by macrophage-mediated processing of OFM.

In Chapter Three, interactions between wound healing cells and OFM were explored. The in vitro studies showed that wound healing cells, FB, endothelial cells and mesenchymal stem cells could be cultured on the material. Although attachment and proliferation was observed in these cultures, the cells did not infiltrate the material the way they would have in an in vivo setting. Evidence in wound healing literature shows that proteases have a vital role in wound healing and biomaterial resorption; therefore, the absence of in vitro infiltration of cells may be due to the absence of proteases. Keratinocytes at the wound edge release proteases to remove cell-cell and cell-matrix attachments, as well as clear matrix debris to allow for migration. In this simple model, cell lines were not stimulated to release proteases as they would do in living tissue. The importance of multiple cells types in model systems has been explored showing that endothelial cells grown on a monolayer of FBs displayed tube forming phenotype as opposed to endothelial cells grown on collagen alone ${ }^{178}$. It is possible that a more complex system, such as FBs and endothelial cells seeded on opposite sides of the OFM for instance, may have led to different cellular behaviours in this model.

In this study the effect of the materials on cell behaviour was investigated. Cell based assays to measure proliferation and migration were chosen as relevant metrics for wound 
healing process. The proliferation of MSC and HUVEC was significantly increased by pepsin and collagenase digested OFM compared with aqueous solutions of OFM after incubation in PBS, media or bicarbonate. This highlights the importance of proteases in the interactions between cells and biomaterials. It was also found that bioactive solutions could be generated from biomaterials made from other ovine tissue including amnion, chorion, dermis, intestine, placental vein and pericardium.

The enzymes used in Chapter Three were readily available, but not necessarily reminiscent of wound healing proteases. Rather than select a number of wound healing enzymes, cells were used to break down the OFM to screen for wound healing bioactives in Chapter Four. Interestingly, macrophages grown on OFM generated a solution that was chemotactic for mesenchymal stem cells. The same was not shown to be true for other cells such as FBs and mesenchymal stem cells. The chemotactic activity of the MO-CM was significantly greater than in the media generated from the same number of macrophages alone and the same mass of OFM. The OFM stained with Congo red was broken down by macrophages. Additionally, the cells removed from Congo red OFM appeared to have taken up the Congo red.

OFM was labelled with FITC to further investigate this bioactive CM in Chapter Five. Proteins derived from the OFM in the media would be fluorescently labelled and other proteins would not be. The CM of three samples were compared by SDS-PAGE separation. M-CM contained a number of unlabelled proteins with a range of sizes on the SDS-PAGE gel. O-CM contained a large fluorescently labelled protein around $100 \mathrm{kDa}$ and a small fluorescent band (less than $5 \mathrm{kDa}$ ) which could have been unbound FITC. The MO-CM contained the same unlabelled bands present in the M-CM sample, the fluorescently labelled bands present in the O-CM sample and a unique $12 \mathrm{kDa}$ fluorescently labelled band. This suggested that an OFM derived protein was going into solution or being cleaved or processed because of macrophages in the culture.

Macrophages in wound healing are known to release a number of chemokines which recruit cells to the wound bed, but macrophage-mediated processing of OFM to release a single distinct protein is new territory. In Chapter Five, the protein of interest was enriched 
from the macrophage proteins in a number of ways. Firstly, the ratio of macrophages to OFM was altered to favour the presence of the unknown protein compared with the macrophage-derived proteins observed in the SDS-PAGE separation of the sample. The culture time was shortened to reduce the expression of macrophage proteins and the volume of cell lysate. Serum was removed from the experiment to reduce noise and improve proteolytic activity. Samples obtained were now composed of one major band that was fluorescently labelled, indicating that the OFM derived protein was the major component of the media. The protein was desalted and enriched by ion exchange and size exclusion gel filtration. This protein was tested for MSC chemotaxis and showed a bioactive response at $10 \mathrm{ng} / \mathrm{mL}$. This indicates that the peptide release from OFM by macrophages may be responsible for bioactivity observed in the MO-CM sample in Chapter Four. A peptide with chemotactic activity towards MSCs could promote the wound healing process.

The identification of this protein was carried out in Chapter Six. Samples were analysed by MALDI and ESI. Spectra were obtained for MALDI but the $\mathrm{m} / \mathrm{z}$ ranges collected did not point to a significant peptide match in the MASCOT database using either ovine, mouse or other mammalian databases. Using liquid chromatography, the sample appeared to be composed of a number of proteins of similar size which did not display distinct peaks. The whole sample, as well as pooled fractions of the size range including the protein of interest, were analysed by ESI. A MASCOT search surrendered four protein matches: keratin, actin, albumin and decorin.

The only likely OFM derived protein uncovered was decorin, however the unknown protein of interest had a mass of $12 \mathrm{kDa}$, less than half that of decorin. Several maps were generated of peptides identified in the sample after digestion with trypsin and gluC. This evidence suggested that the sample may contain one or more fragments of decorin. A match of these peptides with known protease substrate binding sites revealed a number of possible fragments that were similar in size to the protein of interest. 
The bioactivity in terms of MSC chemotaxis was observed on an undiluted sample of MO-

$\mathrm{CM}$ enriched for a $12 \mathrm{kDa}$ peptide within this sample. It is plausible that one or more fragments of decorin could be responsible for the observed bioactivity.

\subsection{Key Findings}

\subsubsection{Proteolytic digestion of dECM biomaterials in wound healing}

The proteolytic cleavage of the ECM is a vital part of wound healing ${ }^{39,78}$. It is possible to list a number of growth factors, cryptic peptides and protein fragments that have been assigned a role in wound healing. One of the first examples of ECM peptide bioactivity is fibronectin. A study in 1982 showed that fragments of this ECM protein were chemotactic for blood monocytes ${ }^{216}$. Peptides from other ECM proteins, such as collagen and elastin have also demonstrated chemotactic properties ${ }^{92,217,218}$.

The first pivotal action of the body in wound healing is the formation of the fibrin clot resulting in the recruitment of a number of cells. This is the first in a series of events shifting resting tissue to an activated state in which cells are highly motile and proliferative. Further along in the process, the breakdown of the temporary granulation tissue and damaged ECM also leads to cell movement.

In terms of stem and progenitor cells, ECM components have been shown to actively recruit these cells to the site of tissue damage ${ }^{63}$. Models have also shown that stem and progenitor cells have been recruited to the site of injury after implantation with a dECM biomaterial $^{1,183}$.

Since dECM materials are composed of these ECM proteins, bioactive components of dECM materials can be found by enzymatic digestion of the materials. Agrawal et al. found peptides derived from SIS collagen that promoted osteogenesis ${ }^{182}$. 


\subsubsection{Decorin as a cryptic peptide in biomaterial facilitated wound healing}

The role of decorin in wound healing is not clear. Some studies have shown that this protein is implicated in angiogenesis ${ }^{135,211,219}$. Most studies have focused on the full length protein but no divergent activity has been assigned to fragments of the decorin. It was also clear that this protein is able to sequester TGF $\beta$, a growth factor that has a number of roles in wound healing. Finally, the leucine rich region of decorin is known to interact with wound healing cells via TGF $\beta$ receptor $220,221$.

\subsection{Limitations and assumptions of the study}

In vitro assays are an effective tool to study the effects of molecules on processes in living tissues, but they are models. The behaviour of cells is clearly different in vivo, as shown by the lack of cellular infiltration of the matrix in Chapter One.

To conclude that OFM is processed by macrophages to release a decorin fragment which directs a chemotactic response in MSCs certain assumptions must be made. However, these significant changes in cellular behaviour merit further investigation.

Firstly, the MO-CM sample displayed greater activity than the M-CM and the O-CM samples. Since the MO-CM sample contains a unique $12 \mathrm{kDa}$ peptide, the increase in activity could be assigned to this peptide. It could also be a combinatory effect of the OCM and M-CM samples. That does not explain why using other cell lines, such as FBs, did not give this peculiar increase in activity even though the FB CM was also bioactive.

Secondly, a cautious approach was taken, enriching samples of unlabelled and labelled MO-CM side by side for fear that modification to the OFM may interfere with macrophage binding, protease substrate availability or bioactivity of the peptide. The enriched peptide observed in the MO-CM sample displayed MSC chemotaxis. The result is encouraging; however, the sample is an enriched one not an isolated sample of the peptide. It would be interesting to investigate the activity of a pure isolated sample of the $12 \mathrm{kDa}$ peptide. One way to show this could be to use a label such as biotin that would completely remove the 
protein of interest from the sample of macrophage proteins. Alternatively, DCN could be digested with MMPs with substrate binding affinity for this protein and the fragments could be collected and tested for activity.

The results in this study implicate decorin as a potential matrikine, processed by macrophage to form chemotactic fragments. Decorin has many proposed roles in wound healing. The protein binds to other ECM proteins, especially collagen where it appears to function in collagen fibrillogenesis. It also sequesters growth factor TGF $\beta$, a known chemokine for FBs and MSCs. It is possible that macrophage processing of decorin results in the release of TGF $\beta$ which causes the observed response. A comparison could be carried out using recombinant decorin, showing whether macrophages process the protein in the same way when it is not assembled in an extracellular matrix and whether the protein is itself the bioactive.

\subsection{Future directions}

Methods used in this study could be used to find other peptides that interact with wound healing cells. There are a number of ways in which the methods used could be modified to achieve this.

\section{Alternative activation of macrophages}

The macrophages in this study were not activated to either M1 or M2 phenotype in these experiments. There is ample evidence that either phenotype is taken on during wound healing, repeating this macrophage-OFM culture with either M1 or M2 activated macrophages could lead to changes in MMP expression and the release of different peptides from the OFM.

\section{Other cells in ECM breakdown}

Other cells could be explored in the CM experiments. Macrophages are not the only leukocytes that release ECM degrading proteases. Mast cells, neutrophils and dendritic 
cells are also important leukocytes in this process. Non-leukocytes also generate MMPs, on a different scale. These experiments could be modified to explore the degradation profile of MSCs, FBs and endothelial cells. These cells may require longer incubation times to degrade ECM peptides, or a greater ratio of cells to ECM. In addition, combinations of cells could be used to this end. The effect of paracrine signalling from MSCs on other wound healing cells may induce a remodelling phenotype.

\section{Analysis of decorin bioactivity}

In order to test whether the breakdown of decorin is required to reveal bioactivity, an experiment with recombinant decorin could be used in the MSC migration assay. Decorin in the ECM has a great affinity for growth factors thus it is possible that some of the activity observed is due to the release of these growth factors after decorin degradation. Further analysis of the CM may show the presence of labile growth factors that were destroyed during the processing of the CM.

\section{Wound healing assays}

The chemotactic CM was only tested on MSCs. It is possible that peptides within this sample also recruit other wound healing cells or participate in other wound healing processes. It would be interesting to test the CM sample with angiogenesis assays and in in vivo wound healing studies.

\subsection{Conclusion}

The most significant findings in this work relate to the interplay between mesenchymal cells, macrophages and OFM. Firstly, more bioactive compounds are revealed after proteolytic digestion of the material than after dissolution into media. Enzymatically digested material promotes proliferation and migration of key wound healing cells. This demonstrates the OFM, and other AECM biomaterials, are actively involved in modifying cell proliferation and migration. This supports a growing body of evidence that ECM materials are able to aid wound healing in an active manner. 
Secondly, macrophages are highly involved in constructive remodelling and express proteases that may release bioactive fragments from the $\mathrm{AECM}$ into solution in vitro. The activity observed here is greater than the activity generated from macrophages alone or OFM alone. This suggests a critical interaction between macrophages and OFM.

Finally, one of the proteins identified in this bioactive sample is the proteoglycan decorin. Analysis of the MMP substrate binding site in decorin and review of proteases expressed by macrophages point towards a bioactive peptide or peptides derived from decorin being present in the sample. 


\section{References}

1. Swinehart, I. T. \& Badylak, S. F. Extracellular matrix bioscaffolds in tissue remodeling and morphogenesis. Dev. Dyn. (2016). doi: 10.1002/dvdy.24379

2. Wilson, S. L., Sidney, L. E., Dunphy, S. E., Rose, J. B. \& Hopkinson, A. Keeping an eye on decellularized corneas: a review of methods, characterization and applications. J. Funct. Biomater. 4, 114-161 (2013).

3. Keatch, R. P., Schor, A. M., Vorstius, J. B. \& Schor, S. L. Biomaterials in regenerative medicine: engineering to recapitulate the natural. Curr. Opin. Biotechnol. 23, 579-582 (2012).

4. Bonnans, C., Chou, J. \& Werb, Z. Remodelling the extracellular matrix in development and disease. Nat. Rev. Mol. Cell Biol. 15, 786-801 (2014).

5. Hynes, R. O. \& Naba, A. Overview of the Matrisome-An Inventory of Extracellular Matrix Constituents and Functions. Cold Spring Harb. Perspect. Biol. 4, a004903 (2012).

6. Rest, M. van der \& Garrone, R. Collagen family of proteins. FASEB J. Off. Publ. Fed. Am. Soc. Exp. Biol. 5, 2814-2823 (1991).

7. Stenzel, K. H., Miyata, T. \& Rubin, A. L. Collagen as a Biomaterial. Annu. Rev. Biophys. Bioeng. 3, 231-253 (1974).

8. Mouw, J. K., Ou, G. \& Weaver, V. M. Extracellular matrix assembly: a multiscale deconstruction. Nat. Rev. Mol. Cell Biol. 15, 771-785 (2014).

9. Duca, L., Floquet, N., Alix, A. J. P., Haye, B. \& Debelle, L. Elastin as a matrikine. Crit. Rev. Oncol. Hematol. 49, 235-244 (2004).

10. Sagar, B., Rentala, S., Gopal, P. N. V., Sharma, S. \& Mukhopadhyay, A. Fibronectin and laminin enhance engraftibility of cultured hematopoietic stem cells. Biochem. Biophys. Res. Commun. 350, 1000-1005 (2006). 
11. Potts, J. R. \& Campbell, I. D. Structure and function of fibronectin modules. Matrix Biol. 15, 313-320 (1996).

12. Clark, R. A. Potential roles of fibronectin in cutaneous wound repair. Arch. Dermatol. 124, 201-206 (1988).

13. Mao, Y. \& Schwarzbauer, J. E. Fibronectin fibrillogenesis, a cell-mediated matrix assembly process. Matrix Biol. 24, (2005).

14. Leavesley, D. I. et al. Vitronectin-Master controller or micromanager? IUBMB Life $65,807-818$ (2013).

15. Clark, R. A. F. in The Molecular and Cellular Biology of Wound Repair (ed. Clark, R. A. F.) 3-50 (Springer US, 1988).

16. Hocking, A., Shinomura, T. \& DJ, M. Leucine-rich repeat glycoproteins of the extracellular matrix. Matrix Biol. J. Int. Soc. Matrix Biol. 17, 1-19 (1998).

17. McEwan, P. A., Scott, P. G., Bishop, P. N. \& Bella, J. Structural correlations in the family of small leucine-rich repeat proteins and proteoglycans. J. Struct. Biol. 155, 294-305 (2006).

18. Kalamajski, S. \& Oldberg, A. The role of small leucine-rich proteoglycans in collagen fibrillogenesis. Matrix Biol. J. Int. Soc. Matrix Biol. 29, 248-253 (2010).

19. Dellett, M., Hu, W., Papadaki, V. \& Ohnuma, S. Small leucine rich proteoglycan family regulates multiple signalling pathways in neural development and maintenance. Dev. Growth Differ. 54, 327-340 (2012).

20. Beaulieu, J. F. Extracellular matrix components and integrins in relationship to human intestinal epithelial cell differentiation. Prog. Histochem. Cytochem. 31, 1-78 (1997)

21. Bissell, M. J., Hall, H. G. \& Parry, G. How does the extracellular matrix direct gene expression? J. Theor. Biol. 99, 31-68 (1982).

22. Bornstein, B. in Pathobiology of the Endothelial Cell 215-228 (Academic Press, 1982).

23. Schultz, G. S., Davidson, J. M., Kirsner, R. S., Bornstein, P. \& Herman, I. M. Dynamic reciprocity in the wound microenvironment. Wound Repair Regen. 19, 134148 (2011) 
24. Huttenlocher, A. \& Horwitz, A. R. Integrins in cell migration. Cold Spring Harb. Perspect. Biol. 3, a005074 (2011).

25. Kim, S.-H., Turnbull, J. \& Guimond, S. Extracellular matrix and cell signalling: the dynamic cooperation of integrin, proteoglycan and growth factor receptor. $J$. Endocrinol. 209, 139-151 (2011).

26. Larsen, M., Artym, V. V., Green, A. J. \& Yamada, K. M. The matrix reorganized: extracellular matrix remodeling and integrin signaling. Curr. Opin. Cell Biol. 18, 463471 (2006).

27. Holst, J. et al. Substrate elasticity provides mechanical signals for the expansion of hemopoietic stem and progenitor cells. Nat. Biotechnol. 28, 1123-1128 (2010).

28. Rhee, S. \& Grinnell, F. Fibroblast mechanics in 3D collagen matrices. Adv. Drug Deliv. Rev. 59, 1299-1305 (2007).

29. Grinnell, F. Fibroblast biology in three-dimensional collagen matrices. Trends Cell Biol. 13, 264269 (2003).

30. Vedrenne, N., Coulomb, B., Danigo, A., Bonté, F. \& Desmoulière, A. The complex dialogue between (myo)fibroblasts and the extracellular matrix during skin repair processes and ageing. Pathol. Biol. (Paris) 60, 20-27 (2012).

31. Gronski, T. J. et al. Hydrolysis of a Broad Spectrum of Extracellular Matrix Proteins by Human Macrophage Elastase. J. Biol. Chem. 272, 12189-12194 (1997).

32. Payne, V. \& Kam, P. C. A. Mast cell tryptase: a review of its physiology and clinical significance. Anaesthesia 59, 695-703 (2004).

33. Brennan, E. P., Tang, X.-H., Stewart-Akers, A. M., Gudas, L. J. \& Badylak, S. F. Chemoattractant activity of degradation products of fetal and adult skin extracellular matrix for keratinocyte progenitor cells. J. Tissue Eng. Regen. Med. 2, 491-498 (2008).

34. Barrientos, S., Stojadinovic, O., Golinko, M. S., Brem, H. \& Marjana, T.-C. Growth factors and cytokines in wound healing. Wound Repair Regen. Off. Publ. Wound Heal. Soc. Eur. Tissue Repair Soc. 16, 585-601 (2008). 
35. Behm, B., Babilas, P., Landthaler, M. \& Schreml, S. Cytokines, chemokines and growth factors in wound healing. J. Eur. Acad. Dermatol. Venereol. JEADV 26, 812820 (2012).

36. Stoker, M. \& Gherardi, E. Regulation of cell movement: the motogenic cytokines. Biochim. Biophys. Acta 1072, 81-8102 (1991).

37. Macri, L., Silverstein, D. \& Clark, R. Growth factor binding to the pericellular matrix and its importance in tissue engineering. Adv. Drug Deliv. Rev. 59, 13661381 (2007).

38. Vaday, G. G. \& Lider, O. Extracellular matrix moieties, cytokines, and enzymes: dynamic effects on immune cell behavior and inflammation. J. Leukoc. Biol. 67, 149$159(2000)$

39. Moali, C. \& Hulmes, D. J. Extracellular and cell surface proteases in wound healing: new players are still emerging. Eur. J. Dermatol. EJD 19, 552-564 (2009).

40. McCarty, S. M., Cochrane, C. A., Clegg, P. D. \& Percival, S. L. The role of endogenous and exogenous enzymes in chronic wounds: a focus on the implications of aberrant levels of both host and bacterial proteases in wound healing. Wound Repair Regen. Off. Publ. Wound Heal. Soc. Eur. Tissue Repair Soc. 20, 125-136 (2012).

41. Konstantinopoulos, P. A., Karamouzis, M. V., Papatsoris, A. G. \& Papavassiliou, A. G. Matrix metalloproteinase inhibitors as anticancer agents. Int. J. Biochem. Cell Biol. 40, 1156-1168 (2007).

42. Nagai, Y., Lapiere, C. M. \& Gross, J. Tadpole collagenase. Preparation and purification. Biochemistry (Mosc.) 5, 3123-3130 (1966).

43. Strodtbeck, F. Physiology of wound healing. Newborn Infant Nurs. Rev. 1, 43-52 (2001)

44. Aller, M.-A., Arias, J.-I., Arraez-Aybar, L.-A., Gilsanz, C. \& Arias, J. Wound healing reaction: A switch from gestation to senescence. World J. Exp. Med. 4, 16 (2014).

45. Hackam, D. J. \& Ford, H. R. Cellular, biochemical, and clinical aspects of wound healing. Surg. Infect. 3 Supp1 1, S23-S35 (2002). 
46. Velnar, T., Bailey, T. \& Smrkolj, V. The wound healing process: an overview of the cellular and molecular mechanisms. J. Int. Med. Res. 37, 1528-1542 (2009).

47. Yamada, K. M. \& Clark, R. A. F. in The Molecular and Cellular Biology of Wound Repair (ed. Clark, R. A. F.) 51-93 (Springer US, 1988).

48. Greaves, N. S., Ashcroft, K. J., Baguneid, M. \& Bayat, A. Current understanding of molecular and cellular mechanisms in fibroplasia and angiogenesis during acute wound healing. J. Dermatol. Sci. 72, 206-217 (2013).

49. Woodley, D. T. in The Molecular and Cellular Biology of Wound Repair (ed. Clark, R. A. F.) 339-354 (Springer US, 1988).

50. Lönnqvist, S., Emanuelsson, P. \& Kratz, G. Influence of acidic pH on keratinocyte function and re-epithelialisation of human in vitro wounds. J. Plast. Surg. Hand Surg. 49, 346-352 (2015).

51. Larjava, H., Salo, T., Haapasalmi, K., Kramer, R. H. \& Heino, J. Expression of integrins and basement membrane components by wound keratinocytes. J. Clin. Invest. 92, 14251435 (1993).

52. Tomasek, J. J., Gabbiani, G., Hinz, B., Chaponnier, C. \& Brown, R. A. Myofibroblasts and mechano-regulation of connective tissue remodelling. Nat. Rev. Mol. Cell Biol. 3, 349-363 (2002).

53. Madri, J. A., Sankar, S. \& Romanic, A. M. in The Molecular and Cellular Biology of Wound Repair (ed. Clark, R. A. F.) 355-371 (Springer US, 1988).

54. Grinnell, F. Mini-Review on the Cellular Mechanisms of Disease Fibroblasts, Myofibroblasts, and Wound Contraction. (1994).

55. Eckes, B., Aumailley, M. \& Krieg, T. in The Molecular and Cellular Biology of Wound Repair (ed. Clark, R. A. F.) 493-512 (Springer US, 1988). at <http://link.springer.com/chapter/10.1007/978-1-4899-0185-9_16>

56. Richardson, R. et al. Adult Zebrafish as a Model System for Cutaneous WoundHealing Research. J. Invest. Dermatol. 133, 1655-1665 (2013).

57. Safferling, K. et al. Wound healing revised: a novel reepithelialization mechanism revealed by in vitro and in silico models. J. Cell Biol. 203, 691-709 (2013). 
58. Martin, P. Wound healing-aiming for perfect skin regeneration. Science 276, 75-81 (1997).

59. Goessling, W. \& North, T. E. Repairing quite swimmingly: advances in regenerative medicine using zebrafish. Dis. Model. Mech. 7, 769-776 (2014).

60. Govindan, J. \& Iovine, M. K. Dynamic remodeling of the extra cellular matrix during zebrafish fin regeneration. Gene Expr. Patterns 19, 21-29 (2015).

61. Xue, M. \& Jackson, C. J. Extracellular Matrix Reorganization During Wound Healing and Its Impact on Abnormal Scarring. Adv. Wound Care 4, 119-136 (2015).

62. Krieg, T. Cell-cell and cell-matrix interactions in the skin-implications for tissue repair and chronic wounds. Bull. Mém. Académie R. Médecine Belg. 393-7; discussion 398 (2010).

63. Volk, S. W., Iqbal, S. A. \& Bayat, A. Interactions of the Extracellular Matrix and Progenitor Cells in Cutaneous Wound Healing. Adv. Wound Care 2, 261-272 (2013).

64. Wilgus, T. A. Growth Factor-Extracellular Matrix Interactions Regulate Wound Repair. Adv. Wound Care 1, 249-254 (2012).

65. Widgerow, A. D. Cellular/extracellular matrix cross-talk in scar evolution and control. Wound Repair Regen. 19, 117-133 (2011).

66. O'Reilly, P. J., Gaggar, A. \& Blalock, J. E. Interfering with extracellular matrix degradation to blunt inflammation. Curr. Opin. Pharmacol. 8, 242-248 (2008).

67. Frey, H., Schroeder, N., Tina, M.-J., Iozzo, R. V. \& Schaefer, L. Biological interplay between proteoglycans and their innate immune receptors in inflammation. FEBS J. $280,2165-2179(2013)$

68. Taylor, K. R. et al. Hyaluronan fragments stimulate endothelial recognition of injury through TLR4. J. Biol. Chem. 279, 17079-17084 (2004).

69. Gao, F. et al. Hyaluronan oligosaccharides promote excisional wound healing through enhanced angiogenesis. Matrix Biol. 29, 107116 (2010).

70. Dicker, K. T. et al. Hyaluronan: A simple polysaccharide with diverse biological functions. Acta Biomater. 10, (2013). 
71. CHEN, J. W. \& ATANGELO, G. Functions of hyaluronan in wound repair. Wound Repair Regen. 7, 79-89 (1999).

72. Stern, R. Devising a pathway for hyaluronan catabolism: are we there yet? Glycobiology 13, 105R-115R (2003).

73. Stern, R., Asari, A. A. \& Sugahara, K. N. Hyaluronan fragments: An informationrich system. Eur. J. Cell Biol. 85, 699-715 (2006).

74. Qian, Y. et al. The effect of hyaluronan on the motility of skin dermal fibroblasts in nanofibrous scaffolds. Int. J. Biol. Macromol. 79, 133-143 (2015).

75. Noble, P. W. Hyaluronan and its catabolic products in tissue injury and repair. Matrix Biol. 21, 25-29 (2002).

76. Slevin, M., Kumar, S. \& Gaffney, J. Angiogenic oligosaccharides of hyaluronan induce multiple signaling pathways affecting vascular endothelial cell mitogenic and wound healing responses. J. Biol. Chem. 277, 41046-41059 (2002).

77. Rooney, P., Wang, M., Kumar, P. \& Kumar, S. Angiogenic oligosaccharides of hyaluronan enhance the production of collagens by endothelial cells. J. Cell Sci. 105 ( Pt 1), 213-218 (1993).

78. McCarty, S. M. \& Percival, S. L. Proteases and Delayed Wound Healing. Adv. Wound Care 2, 438-447 (2013).

79. Qureshi, N., Morrison, D. C. \& Reis, J. Proteasome protease mediated regulation of cytokine induction and inflammation. Biochim. Biophys. Acta BBA - Mol. Cell Res. $1823,2087-2093$ (2012).

80. Matrisian, L. M. The matrix-degrading metalloproteinases. BioEssays 14, 455-463 (1992).

81. Nagase, H., Visse, R. \& Murphy, G. Structure and function of matrix metalloproteinases and TIMPs. Cardiovasc. Res. 69, 562-573 (2006).

82. Visse, R. \& Nagase, H. Matrix Metalloproteinases and Tissue Inhibitors of Metalloproteinases Structure, Function, and Biochemistry. Circ. Res. 92, 827-839 (2003). 
83. Page-McCaw, A., Ewald, A. J. \& Werb, Z. Matrix metalloproteinases and the regulation of tissue remodelling. Nat. Rev. Mol. Cell Biol. 8, 221-233 (2007).

84. Pilcher, B. K. et al. The activity of collagenase-1 is required for keratinocyte migration on a type I collagen matrix. J. Cell Biol. 137, 1445-1457 (1997).

85. Wilgus, T. A., Roy, S. \& McDaniel, J. C. Neutrophils and Wound Repair: Positive Actions and Negative Reactions. Adv. Wound Care 2, 130716103126002 (2013).

86. Swift, M. E., Kleinman, H. K. \& DiPietro, L. A. Impaired wound repair and delayed angiogenesis in aged mice. Lab. Investig. J. Tech. Methods Pathol. 79, 1479-1487 (1999).

87. Toriseva, M. \& Kähäri, V.-M. Proteinases in cutaneous wound healing. Cell. Mol. Life Sci. CMLS 66, 203-224 (2009).

88. Bellon, G., Martiny, L. \& Robinet, A. Matrix metalloproteinases and matrikines in angiogenesis. Crit. Rev. Oncol. Hematol. 49, 203-220 (2004).

89. Wells, J. M., Gaggar, A. \& Blalock, J. E. MMP generated matrikines. Matrix Biol. 44, $122-129$ (2015).

90. Sylvie, R.-B. \& Salza, R. Matricryptins and matrikines: biologically active fragments of the extracellular matrix. Exp. Dermatol. 23, 457-463 (2014).

91. Maquart, F.-X., Pasco, S., Ramont, L., Hornebeck, W. \& Monboisse, J.-C. An introduction to matrikines: extracellular matrix-derived peptides which regulate cell activity Implication in tumor invasion. Crit. Rev. Oncol. Hematol. 49, 199-202 (2004).

92. Ricard-Blum, S. \& Ballut, L. Matricryptins derived from collagens and proteoglycans. Front. Biosci. Landmark Ed. 16, 674-697 (2011).

93. Tran, K. T., Lamb, P. \& Deng, J.-S. Matrikines and matricryptins: Implications for cutaneous cancers and skin repair. J. Dermatol. Sci. 40, 11-20 (2005).

94. Eming, S. A., Krieg, T. \& Davidson, J. M. Inflammation in wound repair: molecular and cellular mechanisms. J. Invest. Dermatol. 127, 514-525 (2007).

95. McCallion, R. L. \& Ferguson, M. W. J. in The Molecular and Cellular Biology of Wound Repair (ed. Clark, R. A. F.) 561-600 (Springer US, 1988). 
96. Naik-Mathuria, B. et al. Age-dependent recruitment of neutrophils by fetal endothelial cells: implications in scarless wound healing. J. Pediatr. Surg. 42, 166-171 (2007).

97. Kolaczkowska, E. \& Kubes, P. Neutrophil recruitment and function in health and inflammation. Nat. Rev. Immunol. 13, 159-175 (2013).

98. Wulff, B. C. et al. Mast Cells Contribute to Scar Formation during Fetal Wound Healing. J. Invest. Dermatol. 132, 458-465 (2011).

99. Riches, D. W. H. in The Molecular and Cellular Biology of Wound Repair (ed. Clark, R. A. F.) 95-141 (Springer US, 1988).

100. Anghelina, M., Krishnan, P., Moldovan, L. \& Moldovan, N. I. Monocytes/macrophages cooperate with progenitor cells during neovascularization and tissue repair: conversion of cell columns into fibrovascular bundles. Am. J. Pathol. 168, 529-541 (2006).

101. Martins, V. L., Caley, M. \& O’Toole, E. A. Matrix metalloproteinases and epidermal wound repair. Cell Tissue Res. 351, 255-268 (2013).

102. Eckes, B., Nischt, R. \& Krieg, T. Cell-matrix interactions in dermal repair and scarring. Fibrogenesis Tissue Repair 3, 4 (2010).

103. Dutta, R. C. \& Dutta, A. K. Cell-interactive 3D-scaffold; advances and applications. Biotechnol. Adv. 27, 334339 (2009).

104. Brown, R. A. In the beginning there were soft collagen-cell gels: towards better 3D connective tissue models? Exp. Cell Res. 319, 2460-2469 (2013).

105. Rocha-Azevedo, B. da, Ho, C.-H. \& Grinnell, F. Fibroblast cluster formation on 3D collagen matrices requires cell contraction dependent fibronectin matrix organization. Exp. Cell Res. 319, 546-555 (2013).

106. Wang, S. et al. Pericytes Regulate Vascular Basement Membrane Remodeling and Govern Neutrophil Extravasation during Inflammation. PLoS ONE 7, e45499 (2012).

107. Meirelles, L., Chagastelles, P. \& Nardi, N. Mesenchymal stem cells reside in virtually all post-natal organs and tissues. J. Cell Sci. 119, 2204-2213 (2006). 
108. Meirelles, L. da, Caplan, A. I. \& Nardi, N. Chapter 12 - Pericytes as the Source of Mesenchymal Stem Cells. Resid. Stem Cells Regen. Ther. (2012). doi: 10.1016/B978$0-12-416012-5.00012-8$

109. Quesenberry, P. \& Levitt, L. Hematopoietic stem cells (third of three parts). N. Engl. J. Med. 301, 868-872 (1979).

110. Bianco, P. Stem cells and bone: a historical perspective. Bone 70, 2-9 (2015).

111. Rekers, Coulter \& Warren. EFfect of transplantation of bone marrow into irradiated animals. Arch. Surg. 60, 635-667 (1950).

112. Thomas, E. D., Lochte, H. L., Lu, W. C. \& Ferrebee, J. W. Intravenous infusion of bone marrow in patients receiving radiation and chemotherapy. N. Engl. J. Med. 257, $491-496$ (1957).

113. Friedenstein, A. J. Osteogenetic activity of transplanted transitional epithelium. Acta Anat. (Basel) 45, 31-59 (1961).

114. Friedenstein, A. J., Chailakhjan, R. K. \& Lalykina, K. S. The development of fibroblast colonies in monolayer cultures of guinea-pig bone marrow and spleen cells. Cell Tissue Kinet. 3, 393-403 (1970).

115. Caplan, A. I. Mesenchymal stem cells. J. Orthop. Res. Off. Publ. Orthop. Res. Soc. 9 , $641-650$ (1991).

116. Caplan, A. I. Adult mesenchymal stem cells for tissue engineering versus regenerative medicine. J. Cell. Physiol. 213, 341-347 (2007).

117. Dominici, M. et al. Minimal criteria for defining multipotent mesenchymal stromal cells. The International Society for Cellular Therapy position statement. Cytotherapy 8, 315-317 (2006).

118. Li, H. \& Fu, X. Mechanisms of action of mesenchymal stem cells in cutaneous wound repair and regeneration. Cell Tissue Res. 348, 371-377 (2012).

119. Sasaki, M. et al. Mesenchymal Stem Cells Are Recruited into Wounded Skin and Contribute to Wound Repair by Transdifferentiation into Multiple Skin Cell Type. $J$. Immunol. 180, 2581-2587 (2008). 
120. Shabbir, A., Cox, A., Rodriguez-Menocal, L., Salgado, M. \& Badiavas, E. V. Mesenchymal Stem Cell Exosomes Induce Proliferation and Migration of Normal and Chronic Wound Fibroblasts, and Enhance Angiogenesis In Vitro. Stem Cells Dev. 24, 1635-1647 (2015).

121. Liu, Z. \& Klominek, J. Chemotaxis and chemokinesis of malignant mesothelioma cells to multiple growth factors. Anticancer Res. 24, 1625-1630 (2004).

122. Lapidot, T., Dar, A. \& Kollet, O. How do stem cells find their way home? Blood 106, $1901-1910$ (2005).

123. Ratajczak, M. Z., Serwin, K. \& Schneider, G. Innate Immunity Derived Factors as External Modulators of the CXCL12 - CXCR4 Axis and Their Role in Stem Cell Homing and Mobilization. Theranostics 3, 3-10 (2013).

124. Evans, N. D., Oreffo, R. O. C., Healy, E., Thurner, P. J. \& Man, Y. H. Epithelial mechanobiology, skin wound healing, and the stem cell niche. J. Mech. Behav. Biomed. Mater. 28, 397-409 (2013).

125. Gawronska-Kozak, B., Grabowska, A., Kopcewicz, M. \& Kur, A. Animal models of skin regeneration. Reprod. Biol. 14, 6167 (2014).

126. Caruso, U., Tisone, G., Cravotto, E., Casciani, C. U. \& D'Iddio, S. Evaluation of liver regeneration after exeresis by determination of plasma carnitine levels in rats. Int. J. Tissue React. 5, 201-203 (1983).

127. Wong, V. W., Gurtner, G. C. \& Longaker, M. T. Wound Healing: A Paradigm for Regeneration. Mayo Clin. Proc. 88, 1022-1031 (2013).

128. Oberwallner, B. et al. Preparation of cardiac extracellular matrix scaffolds by decellularization of human myocardium. J. Biomed. Mater. Res. A 102, 3263-3272 (2014).

129. Feng, G., Hao, D. \& Chai, J. Processing of CXCL12 impedes the recruitment of endothelial progenitor cells in diabetic wound healing. FEBS J. 281, 5054-5062 (2014).

130. Mirza, R. E., Fang, M. M., Weinheimer-Haus, E. M., Ennis, W. J. \& Koh, T. J. Sustained inflammasome activity in macrophages impairs wound healing in type 2 diabetic humans and mice. Diabetes 63, 1103-1114 (2013). 
131. Wynn, T. A. Cellular and molecular mechanisms of fibrosis. J. Pathol. 214, 199-210 (2008).

132. Roma-Lavisse, C. et al. M1 and M2 macrophage proteolytic and angiogenic profile analysis in atherosclerotic patients reveals a distinctive profile in type 2 diabetes. Diab. Vasc. Dis. Res. 12, 279-289 (2015).

133. Haslett, C. \& Henson, P. in The Molecular and Cellular Biology of Wound Repair (ed. Clark, R. A. F.) 143-168 (Springer US, 1988).

134. Koivisto, L., Heino, J., Häkkinen, L. \& Larjava, H. Integrins in Wound Healing. Adv. Wound Care 3, 762-783 (2014).

135. Nelimarkka, L. et al. Decorin is produced by capillary endothelial cells in inflammation-associated angiogenesis. Am. J. Pathol. 158, 345-353 (2001).

136. Groeneveld, T. W. et al. Interactions of the extracellular matrix proteoglycans decorin and biglycan with C1q and collectins. J. Immunol. Baltim. Md 1950 175, $4715-4723(2005)$

137. Arroyo, A. G. \& Iruela-Arispe, L. M. Extracellular matrix, inflammation, and the angiogenic response. Cardiovasc. Res. 86, 226-235 (2010).

138. Leibovich, S. J. Production of macrophage-dependent fibroblast-stimulating activity (M-FSA) by murine macrophages. Effects on BALBc 3T3 fibroblasts. Exp. Cell Res. 113, 47-56 (1978).

139. Leibovich, S. J. \& Ross, R. The role of the macrophage in wound repair. A study with hydrocortisone and antimacrophage serum. Am. J. Pathol. 78, 71-7100 (1975).

140. Daley, J. M., Brancato, S. K., Thomay, A. A., Reichner, J. S. \& Albina, J. E. The phenotype of murine wound macrophages. J. Leukoc. Biol. 87, 59-67 (2010).

141. Murray, P. J. \& Wynn, T. A. Protective and pathogenic functions of macrophage subsets. Nat. Rev. Immunol. 11, 723-737 (2011).

142. Novak, M. L. \& Koh, T. J. Phenotypic Transitions of Macrophages Orchestrate Tissue Repair. Am. J. Pathol. 183, 1352-1363 (2013). 
143. Weidenbusch, M. \& Anders, H.-J. Tissue Microenvironments Define and Get Reinforced by Macrophage Phenotypes in Homeostasis or during Inflammation, Repair and Fibrosis. J. Innate Immun. 4, 463-477 (2012).

144. White, M. J. V. \& Gomer, R. H. Trypsin, Tryptase, and Thrombin Polarize Macrophages towards a Pro-Fibrotic M2a Phenotype. PLOS ONE 10, e0138748 (2015).

145. Mosser, D. M. \& Edwards, J. P. Exploring the full spectrum of macrophage activation. Nat. Rev. Immunol. 8, 958-969 (2008).

146. Koh, T. J. \& DiPietro, L. A. Inflammation and wound healing: the role of the macrophage. Expert Rev. Mol. Med. 13, e23 (2015).

147. Sindrilaru, A. \& Scharffetter-Kochanek, K. Disclosure of the Culprits: MacrophagesVersatile Regulators of Wound Healing. Adv. Wound Care 2, 357-368 (2013).

148. Anderson, J. M., Rodriguez, A. \& Chang, D. T. Foreign body reaction to biomaterials. Semin. Immunol. 20, 86-8100 (2007).

149. Ratner, B. D. The Biocompatibility Manifesto: Biocompatibility for the Twenty-first Century. J Cardiovasc. Transl. Res. 4, 523-527 (2011).

150. Ratner, B. D. \& Bryant, S. J. Biomaterials: Where We Have Been and Where We are Going. Annu. Rev. Biomed. Eng. 6, 41-75 (2004).

151. Ridley, H. Intra-ocular acrylic lenses after cataract extraction. 1952. Bull. World Health Organ. 81, 758-761 (2003).

152. Badylak, S. F. The extracellular matrix as a biologic scaffold material. Biomaterials 28, 3587-3593 (2007).

153. Ratner, B. D. Healing with medical implants: The body battles back. Sci. Transl. Med. 7, 272fs4-272fs4 (2015).

154. Rosenzweig, D. H., Carelli, E., Steffen, T., Jarzem, P. \& Haglund, L. 3D-Printed ABS and PLA Scaffolds for Cartilage and Nucleus Pulposus Tissue Regeneration. Int. J. Mol. Sci. 16, 15118-15135 (2015). 
155. Nishimoto, S. K. \& Nishimoto, M. Matrix Gla Protein Binds to Fibronectin and Enhances Cell Attachment and Spreading on Fibronectin. Int. J. Cell Biol. 2014, 1-13 (2014).

156. Heher, P. et al. A novel bioreactor for the generation of highly aligned 3D skeletal muscle-like constructs through orientation of fibrin via application of static strain. Acta Biomater. 24, 251-265 (2015).

157. Badylak, S. F. Xenogeneic extracellular matrix as a scaffold for tissue reconstruction. Transpl. Immunol. 12, 367-377 (2004).

158. Shevchenko, R. V., James, S. L. \& James, S. E. A review of tissue-engineered skin bioconstructs available for skin reconstruction. J. R. Soc. Interface 7, 229-258 (2010).

159. Chvapil, M. et al. Reaction of vaginal tissue of rabbits to inserted sponges made of various materials. J. Biomed. Mater. Res. 13, 1-13 (1979).

160. Rubin, A. L. et al. Acellular corneal transparency. Nature 230, 120-121 (1971).

161. Badylak, S. F., Lantz, G. C., Coffey, A. \& Geddes, L. A. Small intestinal submucosa as a large diameter vascular graft in the dog. J. Surg. Res. 47, 74-80 (1989).

162. Badylak, S. F. et al. The use of xenogeneic small intestinal submucosa as a biomaterial for Achille's tendon repair in a dog model. J. Biomed. Mater. Res. 29, 977-985 (1995).

163. Clarke, K. M. et al. Intestine Submucosa and Polypropylene Mesh for Abdominal Wall Repair in Dogs. J. Surg. Res. 60, 107-114 (1996).

164. Badylak, S. f., Kropp, B., McPherson, T., Liang, H. \& Snyder, P. w. Small Intestinal Submucosa: A Rapidly Resorbed Bioscaffold for Augmentation Cystoplasty in a Dog Model. Tissue Eng. 4, 379-387 (1998).

165. Badylak, S. F. Decellularized allogeneic and xenogeneic tissue as a bioscaffold for regenerative medicine: factors that influence the host response. Ann. Biomed. Eng. 42, 1517-1527 (2014).

166. Boccafoschi, F. et al. Decellularized biological matrices: an interesting approach for cardiovascular tissue repair and regeneration. J. Tissue Eng. Regen. Med. n/a-n/a (2015). doi: 10.1002/term.2103 
167. Badylak, S. F., Taylor, D. \& Uygun, K. Whole-Organ Tissue Engineering: Decellularization and Recellularization of Three-Dimensional Matrix Scaffolds. Biomed. Eng. 13, 27-53 (2011).

168. Crapo, P. M., Gilbert, T. W. \& Badylak, S. F. An overview of tissue and whole organ decellularization processes. Biomaterials 32, 3233-3243 (2011).

169. Gilbert, T. W., Sellaro, T. L. \& Badylak, S. F. Decellularization of tissues and organs. Biomaterials 27, 3675-3683 (2006).

170. Carey, L. E. et al. In vivo degradation of $14 \mathrm{C}$-labeled porcine dermis biologic scaffold. Biomaterials 35, 8297-8304 (2014).

171. Sicari, B. M. et al. The effect of source animal age upon the in vivo remodeling characteristics of an extracellular matrix scaffold. Biomaterials 33, 5524-5533 (2012).

172. Lun, S. et al. A functional extracellular matrix biomaterial derived from ovine forestomach. Biomaterials 31, 4517-4529 (2010).

173. Irvine, S. M. et al. Quantification of in vitro and in vivo angiogenesis stimulated by ovine forestomach matrix biomaterial. Biomaterials 32, 6351-6361 (2011).

174. Ma, B., Wang, X., Wu, C. \& Chang, J. Crosslinking strategies for preparation of extracellular matrix-derived cardiovascular scaffolds. Regen. Biomater. 1, (2014).

175. Choi, Y. C. et al. Decellularized extracellular matrix derived from porcine adipose tissue as a xenogeneic biomaterial for tissue engineering. Tissue Eng. Part C Methods $18,866-876$ (2012).

176. Bryers, J. D., Giachelli, C. M. \& Ratner, B. D. Engineering biomaterials to integrate and heal: the biocompatibility paradigm shifts. Biotechnol. Bioeng. 109, 1898-1911 (2012).

177. Schaefer, L. Complexity of danger: the diverse nature of damage-associated molecular patterns. J. Biol. Chem. 289, 35237-35245 (2014).

178. Liden, B. A. \& May, B. C. H. Clinical Outcomes Following the Use of Ovine Forestomach Matrix (Endoform Dermal Template) to Treat Chronic Wounds. Adv. Skin Wound Care 26, 164 (2013). 
179. Simcock, J. \& May, B. C. H. Ovine forestomach matrix as a substrate for single-stage split-thickness graft reconstruction. Eplasty 13, e58 (2013).

180. Street, M. et al. Augmentation with an ovine forestomach matrix scaffold improves histological outcomes of rotator cuff repair in a rat model. J. Orthop. Surg. 10, 165 (2015).

181. Hodde, J. P., Record, R. D., Liang, H. A. \& Badylak, S. F. Vascular endothelial growth factor in porcine-derived extracellular matrix. Endothel. J. Endothel. Cell Res. 8, 11-24 (2001).

182. Agrawal, V. et al. An Isolated Cryptic Peptide Influences Osteogenesis and Bone Remodeling in an Adult Mammalian Model of Digit Amputation. Tissue Eng. Part A 17, 3033-3044 (2011).

183. Zantop, T., Gilbert, T. W., Yoder, M. C. \& Badylak, S. F. Extracellular matrix scaffolds are repopulated by bone marrow-derived cells in a mouse model of achilles tendon reconstruction. J. Orthop. Res. 24, 1299-1309 (2006).

184. Sicari, B. M. et al. An acellular biologic scaffold promotes skeletal muscle formation in mice and humans with volumetric muscle loss. Sci. Transl. Med. 6, 234ra58 (2014).

185. Itinteang, T., Vishvanath, A., Day, D. J. \& Tan, S. T. Mesenchymal stem cells in infantile haemangioma. J. Clin. Pathol. 64, 232-236 (2011).

186. Schägger, H. Tricine-SDS-PAGE. Nat. Protoc. 1, 16-22 (2006).

187. Rawlings, N. D., Barrett, A. J. \& Finn, R. Twenty years of the MEROPS database of proteolytic enzymes, their substrates and inhibitors. Nucleic Acids Res. 44, D343D350 (2016).

188. Decaestecker, C., Debeir, O., Ham, P. \& Kiss, R. Can anti-migratory drugs be screened in vitro? A review of $2 \mathrm{D}$ and $3 \mathrm{D}$ assays for the quantitative analysis of cell migration. Med. Res. Rev. 27, 149-176 (2007).

189. Weigelt, B., Ghajar, C. M. \& Bissell, M. J. The need for complex 3D culture models to unravel novel pathways and identify accurate biomarkers in breast cancer. $A d v$. Drug Deliv. Rev. 69, 42-51 (2014). 
190. Song, H.-H. G., Park, K. M. \& Gerecht, S. Hydrogels to model 3D in vitro microenvironment of tumor vascularization. Adv. Drug Deliv. Rev. 79, 19-29 (2014).

191. Czirok, A. Endothelial cell motility, coordination and pattern formation during vasculogenesis. Wiley Interdiscip. Rev. Syst. Biol. Med. 5, 587-602 (2013).

192. Yoshida, A., Anand-Apte, B. \& Zetter, B. R. Differential endothelial migration and proliferation to basic fibroblast growth factor and vascular endothelial growth factor. Growth Factors Chur Switz. 13, 57-64 (1996).

193. Anitua, E., Tejero, R., Alkhraisat, M. H. \& Orive, G. Platelet-rich plasma to improve the bio-functionality of biomaterials. BioDrugs Clin. Immunother. Biopharm. Gene Ther. 27, 97-9111 (2013).

194. Giavaresi, G. et al. Response of human chondrocytes and mesenchymal stromal cells to a decellularized human dermis. BMC Musculoskelet. Disord. 14, 12 (2013).

195. Chen, G., Dong, C., Yang, L. \& Lv, Y. 3D Scaffolds with Different Stiffness but Same Microstructure for Bone Tissue Engineering. ACS Appl. Mater. Interfaces (2015). doi: 10.1021/acsami.5b02662

196. Genovese, L. et al. Cellular Localization, Invasion, and Turnover Are Differently Influenced by Healthy and Tumor-Derived Extracellular Matrix. Tissue Eng. Part A 20, 20052018 (2014).

197. Lo, D. D., Zimmermann, A. S., Nauta, A., Longaker, M. T. \& Lorenz, H. P. Scarless fetal skin wound healing update. Birth Defects Res. Part C Embryo Today Rev. 96, $237-247$ (2012).

198. Diegelmann, R. F., Cohen, I. K. \& Kaplan, A. M. The role of macrophages in wound repair: a review. Plast. Reconstr. Surg. (1981).

199. Ariganello, M. B., Simionescu, D. T., Labow, R. S. \& Lee, J. M. Macrophage differentiation and polarization on a decellularized pericardial biomaterial. Biomaterials 32, 439-449 (2011).

200. Bullers, S. J., Baker, S. C., Ingham, E. \& Southgate, J. The Human TissueBiomaterial Interface: A Role for PPAR $y$-Dependent Glucocorticoid Receptor 
Activation in Regulating the CD163+ M2 Macrophage Phenotype. Tissue Eng. Part A $0,1-12$ (2014).

201. Bueter, C. L. et al. Chitosan but not chitin activates the inflammasome by a mechanism dependent upon phagocytosis. J. Biol. Chem. 286, 35447-35455 (2011).

202. Ariganello, M. B., Labow, R. S. \& Lee, J. M. In vitro response of monocyte-derived macrophages to a decellularized pericardial biomaterial. J. Biomed. Mater. Res. A 93A, 280-288 (2010).

203. Werb, Z., Banda, M. \& Jones, P. Degradation of connective tissue matrices by macrophages. I. Proteolysis of elastin, glycoproteins, and collagen by proteinases isolated from macrophages. J. Exp. Med. 152, 1340-1357 (1980).

204. Strupat, K. Molecular Weight Determination of Peptides and Proteins by ESI and MALDI. Methods Enzymol. 405, 1-36 (2005).

205. Roch, T. et al. Expression pattern analysis and activity determination of matrix metalloproteinase derived from human macrophage subsets. Clin. Hemorheol. Microcirc. 58, 147-158 (2014).

206. Krusius, T. \& Ruoslahti, E. Primary structure of an extracellular matrix proteoglycan core protein deduced from cloned cDNA. Proc. Natl. Acad. Sci. U. S. A. 83, 76837687 (1986).

207. Yang, V. W., LaBrenz, S. R., Rosenberg, L. C., McQuillan, D. \& Höök, M. Decorin is a $\mathrm{Zn2}+$ metalloprotein. J. Biol. Chem. 274, 12454-12460 (1999).

208. Svensson, L., Heinegård, D. \& Oldberg, A. Decorin-binding sites for collagen type I are mainly located in leucine-rich repeats 4-5. J. Biol. Chem. 270, 20712-20716 (1995).

209. Oldberg, A., Antonsson, P., Lindblom, K. \& Heinegård, D. A collagen-binding 59-kd protein (fibromodulin) is structurally related to the small interstitial proteoglycans PG-S1 and PG-S2 (decorin). EMBO J. 8, 2601-2604 (1989).

210. Neill, T., Schaefer, L. \& Iozzo, R. V. Decorin: a guardian from the matrix. Am. J. Pathol. 181, 380-387 (2012). 
211. Järveläinen, $\mathrm{H}$. et al. A role for decorin in cutaneous wound healing and angiogenesis. Wound Repair Regen. Off. Publ. Wound Heal. Soc. Eur. Tissue Repair Soc. 14, 443-452 (2006).

212. Reed, C. C. \& Iozzo, R. V. The role of decorin in collagen fibrillogenesis and skin homeostasis. Glycoconj. J. 19, 249-255 (2002).

213. Imai, K., Hiramatsu, A., Fukushima, D., Pierschcher, M. D. \& Okada, Y. Degradation of decorin by matrix metalloproteinases: identification of the cleavage sites, kinetic analyses and transforming growth factor- $\beta 1$ release. Biochem. J. 322, 809-814 (1997).

214. Barrientos, S., Brem, H., Stojadinovic, O. \& Marjana, T.-C. Clinical application of growth factors and cytokines in wound healing. Wound Repair Regen. Off. Publ. Wound Heal. Soc. Eur. Tissue Repair Soc. 22, 569-578 (2014).

215. Burnouf, T. et al. Blood-derived biomaterials and platelet growth factors in regenerative medicine. Blood Rev. 27, 77-89 (2013).

216. Norris, D. A. et al. Fibronectin fragment(s) are chemotactic for human peripheral blood monocytes. J. Immunol. Baltim. Md 1950 129, 1612-1618 (1982).

217. Marneros, A. G. \& Olsen, B. R. The role of collagen-derived proteolytic fragments in angiogenesis. Matrix Biol. 20, 337-345 (2001).

218. Postlethwaite, A. E., Seyer, J. M. \& Kang, A. H. Chemotactic attraction of human fibroblasts to type I, II, and III collagens and collagen-derived peptides. Proc. Natl. Acad. Sci. 75, 871-875 (1978).

219. Järveläinen, H., Sainio, A. \& Wight, T. N. Pivotal role for decorin in angiogenesis. Matrix Biol. J. Int. Soc. Matrix Biol. 43, 15-26 (2015).

220. Baghy, K., Iozzo, R. V. \& Kovalszky, I. Decorin-TGF $\beta$ axis in hepatic fibrosis and cirrhosis. J. Histochem. Cytochem. Off. J. Histochem. Soc. 60, 262-268 (2012).

221. Kwan, P. O., Ding, J. \& Tredget, E. E. Serum Decorin, IL-1 $\beta$, and TGF- $\beta$ Predict Hypertrophic Scarring Postburn. J. Burn Care Res. Off. Publ. Am. Burn Assoc. (2015). 


\section{Appendices}




\section{Appendix 1: Reagents, materials and resources}

The following table includes a list the name and provider of reagents and equipment used in this study.

\section{CHEMICALS}

2-MERCAPTOETHANOL

ACETONE

ACETONITRILE

ACRYLAMIDE $(40 \%)$

ALCIAN BLUE

ALIZARIN RED

AMMONIUM PERSULFATE

BIOPSY PUNCH

BIORAD GEL SYSTEM

CELL CULTURE PLATES

CENTRICON FILTERS (5,000 MWCO)

COLLAGENASE

CONGO RED

COOMASSIE BLUE, R250

DEAE

DMEM \& DMEM PHENOL RED FREE

DMSO

DTT

ECGS

EOSIN Y

FITC

FOETAL BOVINE SERUM

FORMALIN

GELATIN

GLYCINE

HARRIS' HAEMOTOXYLIN

HEPES BUFFER

HORSE SERUM

\section{PROVIDER}

SIGMA-ALDRICH, MA, USA

SIGMA-ALDRICH, MA, USA

SIGMA-ALDRICH, MA, USA

BIO-RAD, CA, USA

SIGMA-ALDRICH, MA, USA

SIGMA-ALDRICH, MA, USA

BIO-RAD, CA, USA

HEALTHLINK, USA

BIO-RAD, CA, USA

NUNC, THERMOFISHER SCIENTIFIC, MO, USA

MERCK MILLIPORE, GERMANY

SIGMA-ALDRICH, MA, USA

SIGMA-ALDRICH, MA, USA

BIO-RAD, CA, USA

SIGMA-ALDRICH, MA, USA

GIBCO $^{\text {TM }}$, THERMOFISHER SCIENTIFIC, MO, USA

SIGMA-ALDRICH, MA, USA

SIGMA-ALDRICH, MA, USA

SIGMA-ALDRICH, MA, USA

S SIGMA-ALDRICH, MA, USA

SIGMA-ALDRICH, MA, USA

GIBCO $^{\text {TM }}$, THERMOFISHER SCIENTIFIC, MO, USA

SIGMA-ALDRICH, MA, USA

SIGMA-ALDRICH, MA, USA

SIGMA-ALDRICH, MA, USA

SIGMA-ALDRICH, MA, USA

SIGMA-ALDRICH, MA, USA

GIBCO $^{\text {TM }}$, THERMOFISHER SCIENTIFIC, MO, USA 


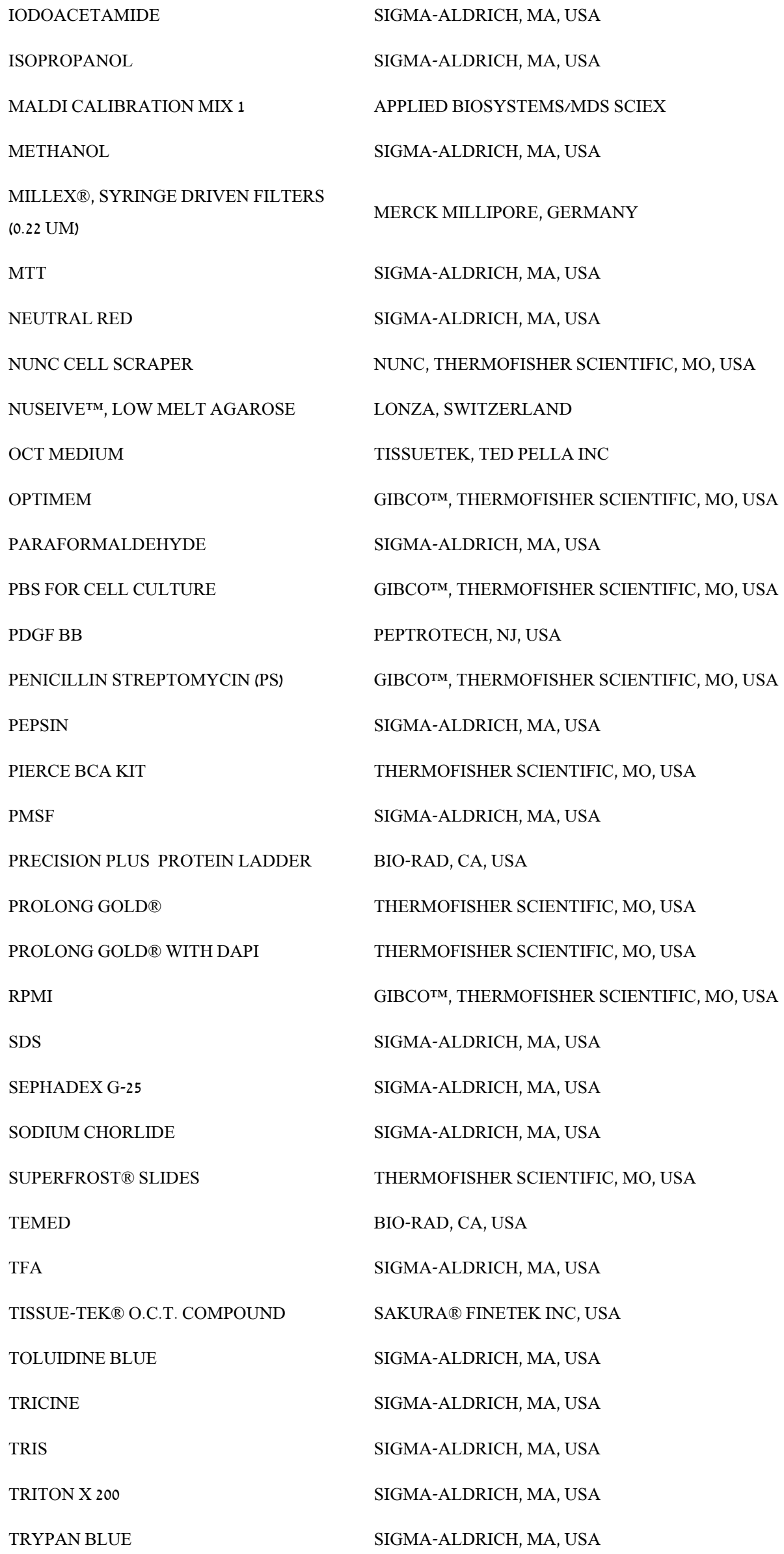


TRYPSIN FOR CELL CULTRE

TRYPSIN FOR MALDI

VEGF

\section{EQUIPMENT}

CRYOSTAT

FLUORESCENT MICROSCOPE

INVERTED MICROSCOPE \& CAMERA

MALDI

PLATE READER
THERMOFISHER SCIENTIFIC, MO, USA

SIGMA-ALDRICH, MA, USA

BECTON DICKINSON, NJ, USA

\section{MAKE AND MODEL}

LEICA CM3050S, LEICA MICROSYSTEMS, HAMBURG, GERMANY

OLYMPUS AX70 PHOTOMICROSCOPE (NORMAL LIGHT, DAPI OR FITC FILTERS) WITH A DP70 CCD CAMERA (OLYMPUS JAPAN)

CKX41 MICROSCOPE WITH A DP12 CAMERA (OLYMPUS, JAPAN) 5800 MALDI TOF/TOF ANALYSER, APPLIED BIOSYSTEMS/MDS SCIEX

PERKIN ELMAR ENSPIRE PLATE READER 


\section{Appendix 2: MSC differentiation}

The mesenchymal stem cell lines used in this study are derived from haemangioma tissue, and are used primarily because they are an easily available source of cells which recapitulate properties of mesenchymal stem cells from human tissue. These cells have been characterised previously and are capable of differentiating down three mesenchymal lineages towards chondrocytes, adipocytes and osteocytes.

In order to establish that the isolated cells would behave in the same way as previously described, cells were grown in differentiating media (osteogenic, adipogenic or chondrogenic) for two weeks, differentiating media is described in Table A-2.

$\begin{array}{lll}\text { ADIPOGENESIS } & \text { OSTEOGENESIS } & \text { CHONDROGENESIS } \\ \text { MEDIA } & \text { MEDIA } & \text { MEDIA }\end{array}$

\begin{tabular}{lll}
\hline DMEM & DMEM + 10 \% FBS & $\begin{array}{l}\text { STEMPRO }(\text { CHONDORGENESIS KIT } \\
\text { (THERMO FISHER) }\end{array}$ \\
DEXAMETHASOME $\left(0.1{ }_{\mathrm{M}} \mathrm{M}\right)$ & DEXAMETHASOME $\left(0.1_{\mathrm{M}} \mathrm{M}\right)$ & \\
ASCORBIC ACID $\left(0.2_{\mathrm{M}} \mathrm{M}\right)$ & ASCORBIC ACID $\left(0.2_{\mathrm{M}} \mathrm{M}\right)$ & \\
B-GLYCEROPHOSPAHTE $\left(10_{\mathrm{M}} \mathrm{M}\right)$ & B-GLYCEROPHOSPHATE $\left(10{ }_{\mathrm{M}} \mathrm{M}\right)$ \\
INSULIN $10 \mu \mathrm{G} / \mathrm{ML}$ & \\
INDOMETHACIN $(60 \mu \mathrm{M})$ &
\end{tabular}

\section{Table A 2: Differentiation media}

After this time cells were fixed and stained for characteristic markers; alizarin red for calcium deposition (osteogenic media), oil red O for lipid vesicles (adipogenic media) and alcian blue for sulphated proteoglycans (chondorgenic media). The MSCs were able to differentiate down all three lineages and did not spontaneously differentiate towards a particular cell type in normal media over the 2 week incubation, as shown in Figure A-1. 
Non-Differentiating Media

A
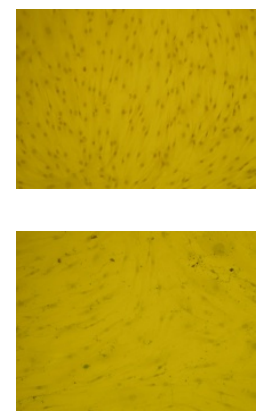

$\mathrm{C}$

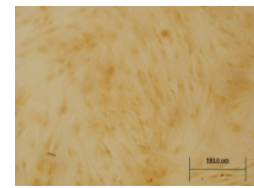

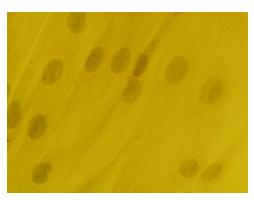
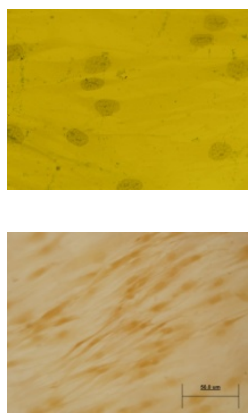

\section{Differentiating Media}
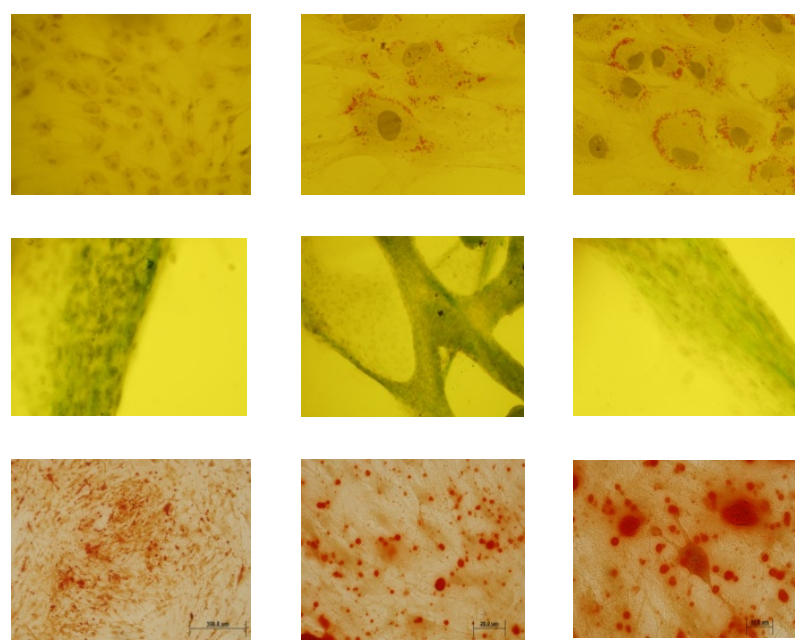
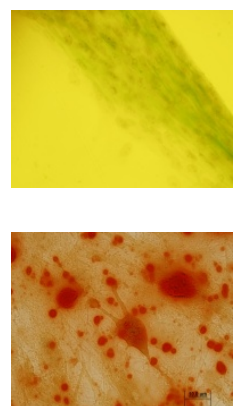

Figure A-1: Differentiation of HDMSCs. Cells were grown for 14 days with and without supplements for adipogenesis, chondrogenesis and osteogenesis following by staining with oil red $\mathrm{O}$ for lipids (A), alcian blue for proteoglycans (B) and alizarin red for calcium deposition (C) respectively. 


\section{Appendix 3: Automated counting of cell-OFM culture cross sections}

Slides of rumen tissue were imaged with DAPI to normalise the automated cell counting of cultures. An image was taken at each magnification (10x, 20x and 40x) to count the number of cells per image and the particle size in pixels (Y). A frequency table was generated for each magnification. Figure A 2 shows the descriptive statistical analysis for this data, giving the $25 \%$ and $75 \%$ percentile values for each image
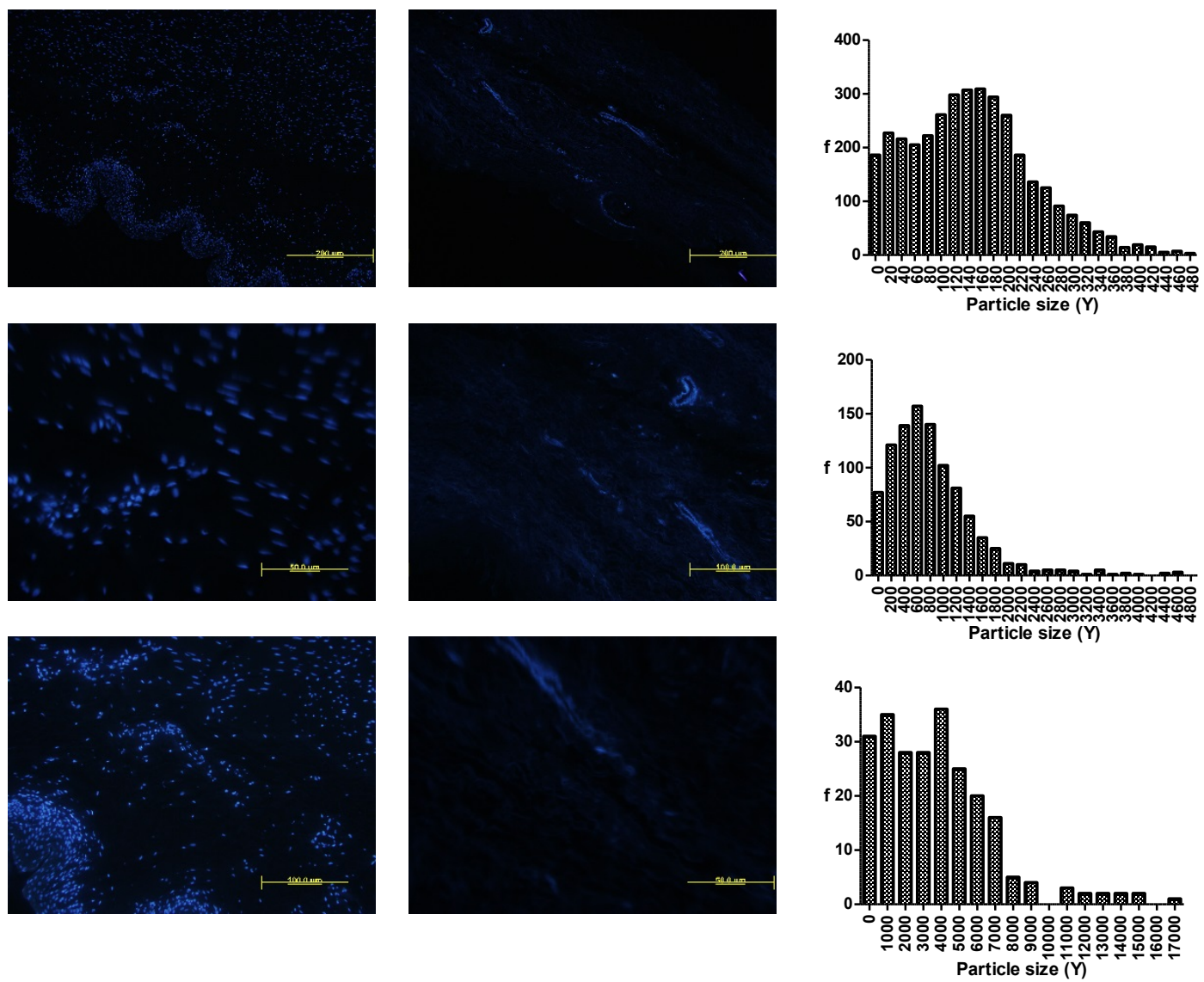

\begin{tabular}{l|cccccc}
\multicolumn{1}{c}{ CELLS } & \multicolumn{5}{c}{ NO CELLS } \\
\hline & $10_{X}$ & $20_{X}$ & $40_{X}$ & $10_{X}$ & $20_{X}$ & $40_{X}$ \\
Total number of values & 3597 & 986 & 240 & 10 & 11 & 4 \\
Minimum & 1.0 & 10.0 & 125.0 & 75.0 & 75.0 & 1835.0 \\
25 \% Percentile & 75.0 & 371.0 & 1345.8 & 80.5 & 81.0 & 1858.5 \\
Median & 142.0 & 697.0 & 3391.0 & 116.0 & 119.0 & 2644.5 \\
75 \% Percentile & 203.0 & 1104.5 & 5321.5 & 146.0 & 149.0 & 4264.5 \\
Maximum & 480.0 & 4671.0 & 16533.0 & 153.0 & 592.0 & 4566.0
\end{tabular}




\begin{tabular}{l|llllll} 
Mean & 146.5 & 826.7 & 3817.7 & 113.6 & 157.0 & 2922.5 \\
Std. Deviation & 92.7 & 671.9 & 3133.3 & 31.6 & 147.3 & 1299.0
\end{tabular}

Figure A 2 Automated cell counting

DAPI stained sections of normal tissue and OFM without cells were used to optimise the automated cell counting. A frequency table was generated for particle sizes collected for images at each magnification 10x, 20x and 40x (top right). For each set of data, the descriptive statistics were generated giving the $25 \%$ to $75 \%$ percentile for the range of particles sizes measured (highlighted). All automated counts were limited to particles sizes within this range for future analysis.

In order to test this system cells were counted manually and by the described automated method. At 20x magnification. As a control decellularised matrix without cells were counted to determine the level of false positive counts. An automated count of matrices with and without cells was compared with a manual count to test the accuracy.

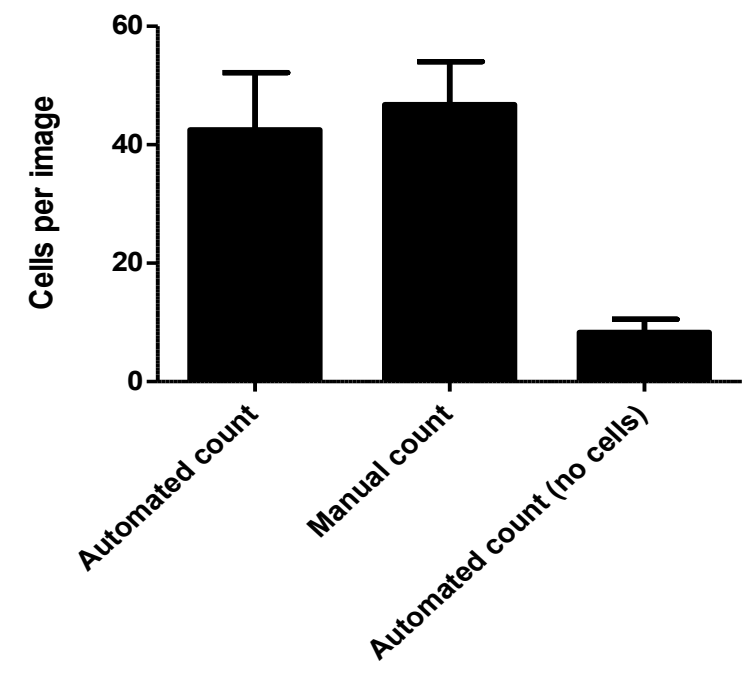

Figure A 3 Automated counting of sections

Automated and Manual counting of DAPI stained sections at 20x magnification. The automated method of cell counting using sections with and without cells was compared with a manual count of the same cell containing section. Error bars indicate the SD between 3 separate images. 
Appendix 4: APAF MALDI and ESI reports 


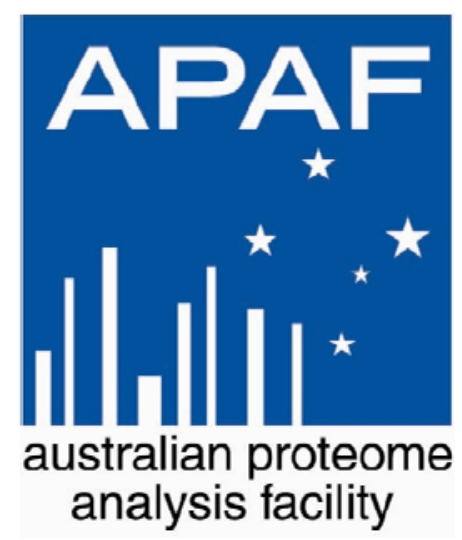

\section{Report: \\ MALDI TOF/ TOF MS analysis for gel spots}

Project code:

Client:

Contact tel:

E-mail:

Report date:

Report prepared by: Matthew Fitzhenry

E-mail:

\section{PROJ 17432}

Sandi Dempsey

Mesynthes Ltd.

Victoria University of Wellington

Kelburn Parade, Kelburn

Wellington

New Zealand

+64 211548088

sandi.dempsey@vuw.ac.nz

$17^{\text {th }}$ February 2015

mfitzhenry@proteome.org.au 
(a) Date samples received:

$9^{\text {th }}$ February 2015

(b) Sample names:

SD10.96.2 (1D gel band)

\section{(c) Instrument used}

Mass Spectrometry: 4800 plus MALDI TOF/TOF Analyser (AB Sciex)

\section{(d) Sample preparation}

Gels were reduced ( $25 \mathrm{mM}$ DTT) at $60^{\circ} \mathrm{C}$ for $30 \mathrm{~min}$ then alkylated $(55 \mathrm{mM}$ IAA) in dark for $20 \mathrm{~min}$. Gels were washed, dried, then digested overnight with $100 \mathrm{ng}$ trypsin. Peptides were extracted with acetonitrile:ammonium bicarbonate, dried, then spotted onto a MALDI plate with $1 \mu \mathrm{L} \mathrm{CHCA} \mathrm{matrix}$ ( $4 \mathrm{mg} / \mathrm{mL}$ ) using an Eppendorf zip-tip (C18 PerfectPure).

\section{(e) Data acquisition}

Matrix Assisted Laser Desorption Ionisation (MALDI) mass spectroscopy was performed using the 4800 plus MALDI TOF/TOF Analyser. A Nd:YAG laser (355 nm) was used to irradiate the sample. Spectra were acquired in reflectron MS scan mode in the mass range of 700 to $4000 \mathrm{Da}$. The instrument was then switched to MS/MS mode where the eight strongest peptides from the MS scan were isolated and fragmented by collisioninduced dissociation, then re-accelerated to measure their masses and intensities. A near point calibration was applied and will give a typical mass accuracy of $50 \mathrm{ppm}$ or better.

\section{(f) Data processing}

The data were exported in a format suitable for submission to the database search program, Mascot (Matrix Science Ltd, London UK). Peaklists were searched against species Ovis aries in the SwissProt database. High scores in the database search indicate a likely match, confirmed or qualified by operator inspection.

\section{(g) Results:}

The monoisotopic peak list in text format and MS scan spectra, Mascot search result are attached.

No proteins were identified from this sample 


\section{Notes}

In the MALDI MS spectrum, please note that four peaks do not belong to the sample.

\section{Acknowledgment}

Any publication arise from this work should be acknowledged the contribution of APAF with the words "This work was undertaken at APAF the infrastructure provided by the Australian Government through the National Collaborative Research I nfrastructure Strategy (NCRIS)."

We welcome your feedback on our services. To go into our quarterly draw to win a prepaid $\$ 200$ debit card follow the link and complete a 5 min questionaire.

https://mqedu.qualtrics.com/SE/?SID=SV 3yIN4uQfHDqOFmZ 


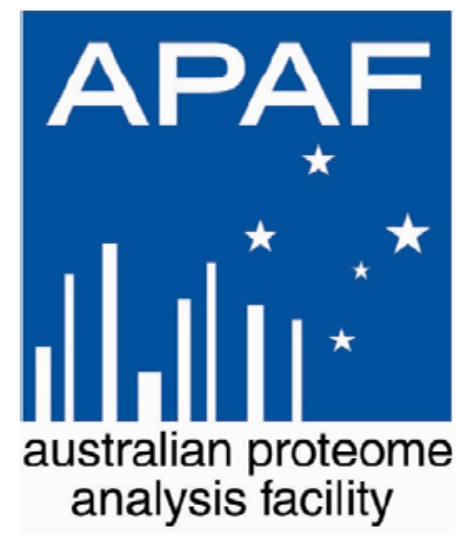

\section{Report: \\ ID nanoLC ESI MS/ MS}

Project code:

PROJ 17432

Client:

Sandi Dempsey

Mesynthes Ltd.

Victoria University of Wellington

Kelburn Parade, Kelburn

Wellington

New Zealand

Contact tel:

+6421 1548088

E-mail:

sandi.dempsey@vuw.ac.nz

Report date:

$17^{\text {th }}$ February 2015

Report prepared by: Matthew Fitzhenry

E-mail:

mfitzhenry@proteome.org.au

APAF - Australia's premier proteomics provider since 1995 
(a) Date samples received:

$9^{\text {th }}$ February 2015

(b) Sample names:

SD10.96.1 (lyophilised solid)

\section{(c) I nstruments used}

Mass spectrometer: Triple TOF 5600 (AB Sciex)

NanoLC system: Eksigent Ultra nanoLC system (Eksigent)

Analytical column: Halo C18, $160 \AA, 2.7 \mu \mathrm{m}, 75 \mu \mathrm{m} \times 10 \mathrm{~cm}$

\section{(d) Sample preparation}

Sample was reduced with DTT $(10 \mathrm{mM}, 20 \mu \mathrm{L})$ for $1 \mathrm{~h}$ at $60^{\circ} \mathrm{C}$, then alkylated with IAA $(20 \mathrm{mM}, 20 \mu \mathrm{L})$ for $30 \mathrm{~min}$ in dark at room temperature. Proteins were digested with $100 \mathrm{ng}$ of trypsin overnight. Sample was concentrated to $30 \mu \mathrm{L}$.

\section{(e) NanoLC ESI MS/ MS data acquisition}

$10 \mu \mathrm{L}$ of sample was injected onto a peptide trap (Michrome peptide Captrap) for pre-concentration and desalted with $0.1 \%$ formic acid, $2 \%$ $\mathrm{ACN}$, at $10 \mu \mathrm{L} / \mathrm{min}$ for 5 minutes. The peptide trap was then switched into line with the analytical column. Peptides were eluted from the column using a linear solvent gradient, with steps, from mobile phase A: mobile phase $B(95: 5)$ to mobile phase $A$ :mobile phase $B(60: 40)$ where mobile phase $A$ is $0.1 \%$ formic acid and mobile phase $B$ is $99.9 \% A C N / 0.1 \%$ formic acid at $550 \mathrm{~nL} / \mathrm{min}$ over a 42 min period. After peptide elution, the column was cleaned with $95 \%$ buffer B for 10 minutes and then equilibrated with buffer $A$ for 25 minutes before next sample injection.

The reverse phase nanoLC eluent was subject to positive ion nanoflow electrospray analysis in an information dependant acquisition mode (IDA). In IDA mode a TOFMS survey scan was acquired (m/z $350-1500,0.25$ 
second), with the ten most intense multiply charged ions (counts $>150$ ) in the survey scan sequentially subjected to MS/MS analysis. MS/MS spectra were accumulated for 200 milliseconds in the mass range m/z $100-1500$ with the total cycle time 2.3 seconds.

\section{(f) Data processing}

The raw data files (.wiff) were converted to mascot generic files (.mgf) using AB SCIEX CommandDriver software. They were submitted to Mascot (Matrix Science, UK) and searched against Swissprot database (Ovis aries)

\section{Acknowledgment}

Any publication arise from this work should be acknowledged the contribution of APAF with the words "This work was undertaken at APAF the infrastructure provided by the Australian Government through the National Collaborative Research I nfrastructure Strategy ( NCRIS)."

We welcome your feedback on our services. To go into our quarterly draw to win a prepaid $\$ 200$ debit card follow the link and complete a 5 min questionaire. 


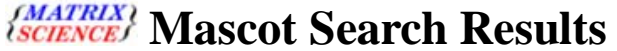

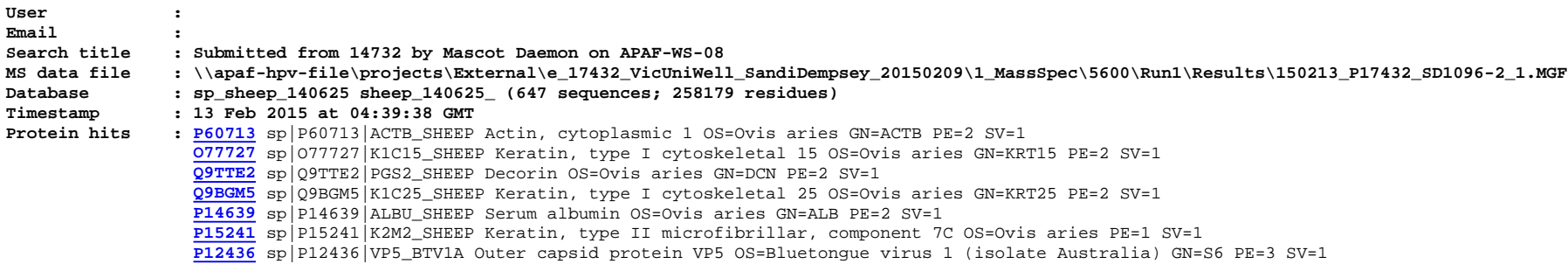

Peptide matches above identity threshold

sp_sheep_140625 Decoy False discovery rate

Peptide matches above homology or identity threshold

$\begin{array}{lll}22 & 4 & 18.18 \% \\ 22 & 4 & 18.18 \%\end{array}$

\section{Mascot Score Histogram}

Ions score is $-10^{*} \log (\mathrm{P})$, where $\mathrm{P}$ is the probability that the observed match is a random event Individual ions scores $>11$ indicate identity or extensive homology $(\mathrm{p}<0.05)$.

Protein scores are derived from ions scores as a non-probabilistic basis for ranking protein hits.

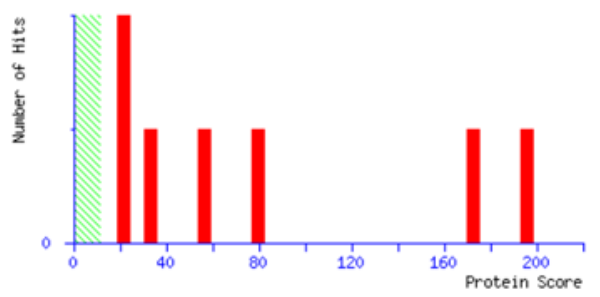

Peptide Summary Report

\begin{tabular}{rll} 
Format As & Peptide Summary & \\
\cline { 1 - 2 } & Significance threshold $\mathrm{p}<0.05$ & Max. number of hits AUTO \\
& Standard scoring $\odot$ MudPIT scoring $\bullet$ & Ions score or expect cut-off 11 \\
Show pop-ups $*$ Suppress pop-ups $\odot$ & Sort unassigned Decreasing Score
\end{tabular}

Help

Show Percolator scores

Show sub-sets 0

Require bold red $\nabla$

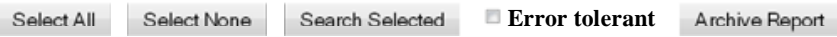

1. P60713 Mass: 41710 Score: 196 Matches: $6(6)$ Sequences: $3(3)$ emPAI: 0.36

sp|P60713|ACTB_SHEEP Actin, cytoplasmic 1 OS=0vis aries GN=ACTB PE=2 SV=1

$\square$ Check to include this hit in error tolerant search or archive report

Query observed $\operatorname{Mr}($ expt) $\operatorname{Mr}(\mathrm{calc}) \quad \mathrm{ppm}$ Miss Score Expect Rank Unique Peptide

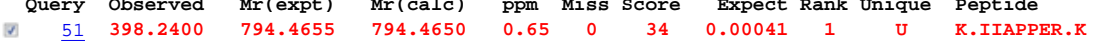

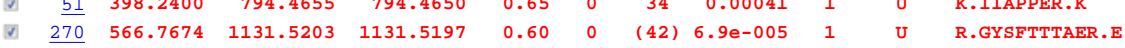

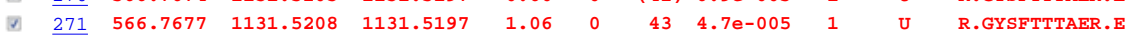

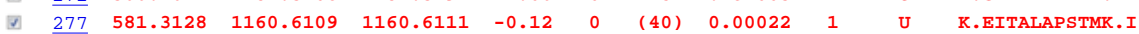

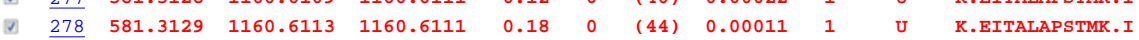

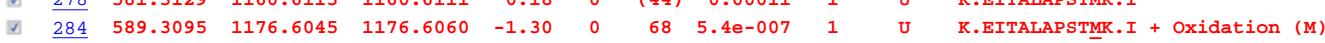

2. 077727 Mass: 48740 Score: 174 Matches: $6(6)$ Sequences: 2(2) emPAI: 0.14 sp|077727|K1C15_SHEEP Keratin, type I cytoskeletal 15 0S=0vis aries GN=KRT15 PE=2 SV=1

Check to include this hit in error tolerant search or archive report

\begin{tabular}{|c|c|c|c|c|c|c|c|c|c|c|}
\hline Query & Observed & $\operatorname{Mr}(\operatorname{expt})$ & $\operatorname{Mr}($ calc) & ppm & Miss & Score & Expect & Rank & Unique & Peptide \\
\hline ․ $\quad \underline{6}$ & 404.2031 & 806.3916 & 806.3923 & -0.86 & 0 & (38) & 0.00015 & 1 & U & R. LAADDFR . L \\
\hline$\underline{57}$ & 404.2036 & 806.3926 & 806.3923 & 0.43 & 0 & 48 & $1.9 e-005$ & 1 & u & R. LAADDFR . L \\
\hline$\underline{58}$ & 404.2073 & 806.4000 & 806.3923 & 9.66 & $\theta$ & (27) & 0.0081 & 1 & u & R. LAADDFR . L \\
\hline 241 & 516.2998 & 1030.5851 & 1030.5910 & -5.70 & 0 & (27) & 0.0034 & 1 & U & R.VLDELTLTK. T \\
\hline$\underline{242}$ & 516.3029 & 1030.5913 & 1030.5910 & 0.26 & 0 & 71 & $7.3 e-008$ & 1 & u & R. VLDELTLTK. T \\
\hline 243 & 516.3036 & 1030.5926 & 1030.5910 & 1.52 & 0 & (34) & 0.00043 & 1 & u & R. VLDELTLTK. T \\
\hline
\end{tabular}

3. Q9TTE2 Mass: 39947 Score: 84 Matches: $2(2)$ Sequences: $1(1)$ emPAI: 0.08 sp|Q9TTE2|PGS2_SHEEP Decorin OS=0vis aries $\mathrm{GN}=\mathrm{DCN}$ PE=2 SV=1

Check to include this hit in error tolerant search or archive report

Query observed $\operatorname{Mr}($ expt) $\operatorname{Mr}($ calc) ppm Miss Score Expect Rank Unique Peptide

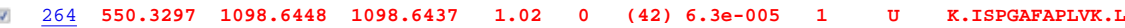

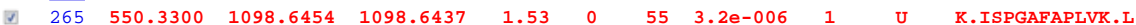

4. Q9BGM5 Mass: 49282 Score: 54 Matches: $4(3)$ Sequences: $2(2)$ emPAI: 0.14 sp|Q9BGM5|K1C25 SHEEP Keratin, type I cytoskeletal 25 OS=0vis aries GN=KRT25 PE=2 SV=1 $\square$ Check to include this hit in error tolerant search or archive report

\begin{tabular}{|c|c|c|c|c|c|c|c|c|c|c|}
\hline Query & Observed & $\operatorname{Mr}(\operatorname{expt})$ & $\operatorname{Mr}($ calc) & ppm & Miss & Score & Expect & Rank & Unique & Peptide \\
\hline$\underline{241}$ & 516.2998 & 1030.5851 & 1030.5845 & 0.63 & 0 & (11) & 0.12 & 2 & u & R.VLDVITLCR.T \\
\hline 242 & 516.3029 & 1030.5913 & 1030.5845 & 6.58 & 0 & (13) & 0.047 & 2 & $\mathrm{u}$ & R.VLDVITLCR.T \\
\hline 243 & 516.3036 & 1030.5926 & 1030.5845 & 7.85 & 0 & 14 & 0.041 & 2 & u & R.VLDVITLCR.T \\
\hline 263 & 545.7676 & 1089.5207 & 1089.5237 & -2.71 & 0 & 53 & $4.8 \mathrm{e}-006$ & 1 & U & K. VTMQNLNDR . L \\
\hline
\end{tabular}


5. P14639 Score: 28 Matches: 1(1) Sequences: 1(1) emPAI: 0.05

$\mathrm{sp}|\mathrm{P} 14639| \mathrm{ALBU}$ SHEEP Serum albumin OS=0vis aries $\mathrm{GN}=\mathrm{ALB}$ PE=2 SV=1

- Check to include this hit in error tolerant search or archive report

Query observed $\operatorname{Mr}$ (expt) $\operatorname{Mr}(\mathrm{calc}) \quad$ ppm Miss Score Expect Rank Unique Peptide

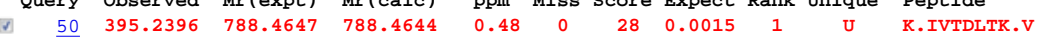

6. P15241 Mass: 53647 Score: 22 Matches: 1(1) Sequences: $1(1)$ emPAI: 0.06

Sp|P15241|K2M2 SHEEP Keratin, type II microfibrillar, component 7C 0S=0vis aries PE=1 SV=1

$\square$ Check to include this hit in error tolerant search or archive report

Query observed $\operatorname{Mr}($ expt) $\operatorname{Mr}($ calc $) \quad$ ppm Miss Score Expect Rank Unique Peptide

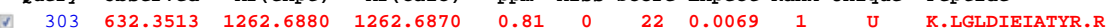

Proteins matching the same set of peptides:

P25691 Mass: 55220 Score: 22 Matches: 1(1) Sequences: $1(1)$

sp|P25691|K2M3 SHEEP Keratin, type II microfibrillar, component 5 OS=0vis aries PE=1 SV=1

7. P12436 Mass: 59207 Score: 22 Matches: $6(5)$ Sequences: $1(1)$ emPAI: 0.06

sp|P12436|VP5_BTV1A Outer capsid protein VP5 OS=Bluetongue virus 1 (isolate Australia) GN=S6 PE=3 SV=1

- Check to include this hit in error tolerant search or archive report

Query observed $\operatorname{Mr}$ (expt) $\operatorname{Mr}$ (calc)

1 $\underline{329} \quad 598.9439 \quad 1793.8098 \quad 1793.7924$

ᄀ $\quad \begin{array}{rrrr}330 & 598.9439 & 1793.8098 & 1793.7924\end{array}$

$7 \quad 332 \quad 598.9442 \quad 1793.8107 \quad 1793.7924$

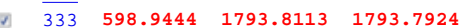

v $\underline{\underline{334}} \quad 598.9444 \quad 1793.8113 \quad 1793.7924$

ppm Miss Score Expect Rank Unique Peptide

$9.7200(15) \quad 0.033 \quad 1 \quad$ U R.DGMQEEAIQEIAGMTR.G + 0xidation (M)

$\begin{array}{lllllll}9.72 & 0 & (15) & 0.03 & 1 & \text { U } & \text { R.DGMQEEAIQEIAGMTR.G + 0xidation (M) }\end{array}$

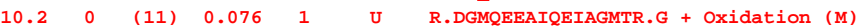

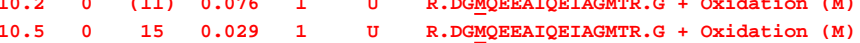

I) $\quad 335 \quad 598.9444 \quad 1793.8113 \quad 1793.7924$

$10.5 \quad 0 \quad(14) \quad 0.038$

R.DGMQEEAIQEIAGMTR.G + Oxidation (M)

Peptide matches not assigned to protein hits: (no details means no match)

\begin{tabular}{|c|c|c|c|}
\hline Query & Observed & $\operatorname{Mr}(\operatorname{expt})$ & $\operatorname{Mr}($ calc) \\
\hline 131 & 442.7633 & 883.5121 & 883.5491 \\
\hline 328 & 897.9110 & 1793.8075 & 1793.79 \\
\hline 331 & 598.9439 & 1793.8098 & 1793.79 \\
\hline 222 & 487.2698 & 972.5250 & 972.51 \\
\hline 217 & 481.2473 & 960.4801 & 960.44 \\
\hline 181 & 457.2951 & 912.5756 & 912.56 \\
\hline 250 & 523.2846 & 1044.5546 & 1044.58 \\
\hline 312 & 681.8625 & 1361.7104 & 1361.75 \\
\hline 327 & 897.9110 & 1793.8074 & 1793.79 \\
\hline 30 & 379.2113 & 756.4080 & 756.39 \\
\hline$\underline{276}$ & 576.2876 & 1150.5606 & 1150.55 \\
\hline 31 & 379.2168 & 756.4191 & 756.3 \\
\hline$\underline{115}$ & 435.7553 & 1738.9921 & 1738.92 \\
\hline 223 & 487.2707 & 972.5268 & 972.5 \\
\hline 53 & 400.7326 & 799.4507 & 799.4 \\
\hline 306 & 643.8787 & 1285.7428 & 1285.7 \\
\hline 209 & 477.3051 & 952.5956 & 952.5 \\
\hline 227 & 494.3017 & 986.5889 & 986.5 \\
\hline 189 & 464.2524 & 926.4902 & 926.4 \\
\hline$\underline{55}$ & 403.2309 & 1206.6708 & 1206.6 \\
\hline 85 & 416.2503 & 830.4860 & 830.4 \\
\hline 207 & 472.5869 & 1414.7390 & 1414.7 \\
\hline 282 & 583.2946 & 1164.5746 & 1164.5 \\
\hline 210 & 477.3053 & 952.5960 & 952.5 \\
\hline$\underline{89}$ & 420.2481 & 838.4816 & 838.4 \\
\hline 84 & 416.2407 & 1245.7002 & 1245.6 \\
\hline 204 & 472.2870 & 942.5594 & 942.5 \\
\hline$\underline{1}$ & 350.1971 & 698.3796 & \\
\hline$\underline{2}$ & 350.2004 & 698.3863 & \\
\hline$\underline{3}$ & 350.2039 & 698.3933 & \\
\hline$\underline{4}$ & 350.2056 & 698.3967 & \\
\hline$\underline{5}$ & 354.0738 & 706.1331 & \\
\hline$\underline{6}$ & 355.0709 & 708.1272 & \\
\hline$\underline{7}$ & 355.1950 & 708.3755 & \\
\hline$\underline{8}$ & 362.1218 & 722.2291 & \\
\hline$\underline{9}$ & 362.1947 & 722.3748 & \\
\hline 10 & 362.2214 & 722.4283 & \\
\hline 11 & 362.2220 & 722.4294 & \\
\hline 12 & 362.2226 & 722.4306 & \\
\hline$\underline{13}$ & 362.2226 & 722.4307 & \\
\hline 14 & 362.2229 & 722.4312 & \\
\hline$\underline{15}$ & 364.2262 & 726.4379 & \\
\hline 16 & 368.0537 & 734.0929 & \\
\hline 17 & 368.0947 & 734.1748 & \\
\hline 18 & 368.1012 & 734.1879 & \\
\hline 19 & 369.1918 & 736.3690 & \\
\hline 20 & 369.9351 & 737.8556 & \\
\hline 21 & 370.7346 & 739.4547 & \\
\hline$\underline{22}$ & 372.2014 & 742.3883 & \\
\hline$\underline{23}$ & 372.2020 & 742.3893 & \\
\hline 24 & 373.1478 & 744.2810 & \\
\hline 25 & 374.0657 & 746.1168 & \\
\hline 26 & 376.0835 & 750.1524 & \\
\hline 27 & 377.0885 & 752.1624 & \\
\hline$\underline{28}$ & 377.8027 & 753.5909 & \\
\hline$\underline{29}$ & 378.1180 & 754.2215 & \\
\hline 32 & 381.2295 & 760.4444 & \\
\hline 33 & 381.2297 & 760.4449 & \\
\hline
\end{tabular}

\begin{tabular}{|c|c|c|c|c|}
\hline ppm & Miss & Score & Expect & \\
\hline 41.85 & 0 & 9 & 0.13 & \\
\hline 8.43 & 0 & 8 & 0.16 & \\
\hline 9.72 & 0 & 8 & 0.17 & \\
\hline 7.75 & 1 & 7 & 0.18 & \\
\hline 32.6 & 0 & 7 & 0.58 & \\
\hline 12.3 & 0 & 7 & 0.19 & \\
\hline 30.29 & 1 & 7 & 0.19 & \\
\hline 33.06 & 1 & 7 & 0.23 & \\
\hline 8.38 & 0 & 7 & 0.2 & \\
\hline 16.9 & 0 & 6 & 0.53 & \\
\hline 8.72 & 0 & 6 & 0.32 & \\
\hline 31.6 & 0 & 6 & 0.57 & \\
\hline 37.6 & 1 & 5 & 0.46 & \\
\hline 9.60 & 1 & 5 & 0.57 & \\
\hline 20.9 & 0 & 4 & 0.35 & \\
\hline 14.5 & 1 & 4 & 0.44 & \\
\hline 26.4 & 0 & 3 & 0.49 & \\
\hline 38.5 & 1 & 3 & 0.53 & \\
\hline 19.4 & 1 & 3 & 1.1 & \\
\hline 45.0 & 1 & 2 & 1.9 & \\
\hline 30.1 & 0 & 2 & 1.5 & \\
\hline 2.77 & 1 & 2 & 0.63 & \\
\hline 41.1 & 0 & 2 & 0.92 & \\
\hline 26.7 & 0 & 2 & 0.67 & \\
\hline 31.9 & 0 & 1 & 0.73 & \\
\hline 7.87 & 1 & 0 & 3 & \\
\hline 1.70 & 1 & 0 & 0.91 & \\
\hline
\end{tabular}

Peptide

IITPAITR

DGMQEEAIQEIAGMTR + 0xidation (M)

DGMOEEAIQEIAGMTR + oxidation (M)

NGAPMTRVK

DLPQMWR + Oxidation (M)

VLLSSPAVK

LSRLQCLR + Carbamidomethyl (C)

QKSQLILEEFK

DGMQEEAIQEIAGMTR + Oxidation (M)

MPPSGLR

YLAEVAAGDDK

MPPSGLR

VVSDPQGIRAWVAWR

NGAPMTRVK

SKLAGLEEALQK

LLEVLNPR

TSLKTPGGAR

YRAYOAR

SEKMLLEDVK + Oxidation (M)

SQLSTQLR

SSMSGLHLVKQGR + Oxidation (M)

IEAMLECQR + Carbamidomethyl (C); 0xidation (M)

LLEVLNPR

KPSDPAPK

QMIQVVKWAK + Oxidation (M)

GNKILVSGR 


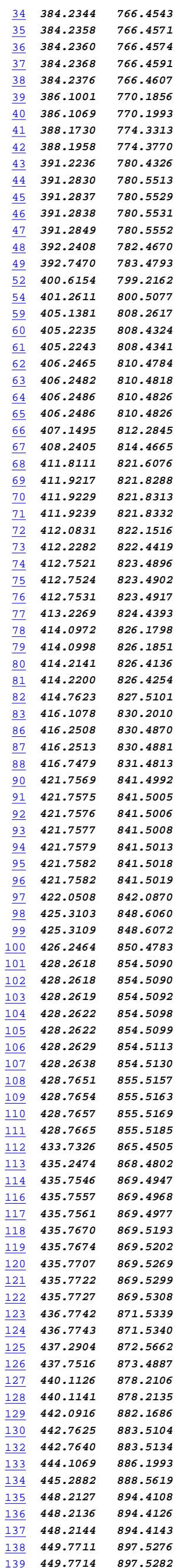

\begin{tabular}{|c|c|c|c|}
\hline 7 & $\overline{35}$ & 384.2358 & 766.4571 \\
\hline च & $\underline{36}$ & 384.2360 & 766.4574 \\
\hline จ & $\overline{37}$ & 384.2368 & 766.4591 \\
\hline$\nabla$ & $\underline{38}$ & 384.2376 & 766.4607 \\
\hline$\nabla$ & 39 & 386.1001 & 770.1856 \\
\hline$\nabla$ & $\underline{40}$ & 386.1069 & 770.1993 \\
\hline v & $\overline{41}$ & 388.1730 & 774.3313 \\
\hline$\nabla$ & $\underline{42}$ & 388.1958 & 774.3770 \\
\hline v & $\underline{43}$ & 391.2236 & 780.4326 \\
\hline$\nabla$ & 44 & 391.2830 & 780.5513 \\
\hline v & 45 & 391.2837 & 780.5529 \\
\hline v & $\underline{46}$ & 391.2838 & 780.5531 \\
\hline v & $\underline{47}$ & 391.2849 & 780.5552 \\
\hline จ & $\overline{48}$ & 392.2408 & 782.4670 \\
\hline च & $\underline{49}$ & 392.7470 & 783.4793 \\
\hline च & $\overline{52}$ & 400.6154 & 799.2162 \\
\hline v & $\underline{54}$ & 401.2611 & 800.5077 \\
\hline$\nabla$ & $\overline{59}$ & 405.1381 & 808.2617 \\
\hline v & 60 & 405.2235 & 808.4324 \\
\hline จ & $\overline{61}$ & 405.2243 & 808.4341 \\
\hline च & $\underline{62}$ & 406.2465 & 810.4784 \\
\hline v & $\overline{63}$ & 406.2482 & 810.4818 \\
\hline v & 64 & 406.2486 & 810.4826 \\
\hline v & 65 & 406.2486 & 810.4826 \\
\hline จ & $\underline{66}$ & 407.1495 & 812.2845 \\
\hline จ & 67 & 408.2405 & 814.4665 \\
\hline v & 68 & 411.8111 & 821.6076 \\
\hline च & $\underline{69}$ & 411.9217 & 821.8288 \\
\hline v & $\overline{70}$ & 411.9229 & 821.8313 \\
\hline च & 71 & 411.9239 & 821.8332 \\
\hline v & $\overline{72}$ & 412.0831 & 822.1516 \\
\hline च & $\underline{73}$ & 412.2282 & 822.4419 \\
\hline$\nabla$ & $\overline{74}$ & 412.7521 & 823.4896 \\
\hline v & $\underline{75}$ & 412.7524 & 823.4902 \\
\hline v & $\underline{76}$ & 412.7531 & 823.4917 \\
\hline v & $\underline{77}$ & 413.2269 & 824.4393 \\
\hline च & $\overline{78}$ & 414.0972 & 826.1798 \\
\hline च & $\underline{79}$ & 414.0998 & 826.1851 \\
\hline v & 80 & 414.2141 & 826.4136 \\
\hline च & 81 & 414.2200 & 826.4254 \\
\hline v & 82 & 414.7623 & 827.5101 \\
\hline v & 83 & 416.1078 & 830.2010 \\
\hline v & 86 & 416.2508 & 830.4870 \\
\hline 8 & $\overline{87}$ & 416.2513 & 830.4881 \\
\hline v & 88 & 416.7479 & 831.4813 \\
\hline v & $\underline{90}$ & 421.7569 & 841.4992 \\
\hline v & 91 & 421.7575 & 841.5005 \\
\hline v & $\overline{92}$ & 421.7576 & 841.5006 \\
\hline च & $\underline{93}$ & 421.7577 & 841.5008 \\
\hline v & $\overline{94}$ & 421.7579 & 841.5013 \\
\hline v & 95 & 421.7582 & 841.5018 \\
\hline च & $\underline{96}$ & 421.7582 & 841.5019 \\
\hline च & 97 & 422.0508 & 842.0870 \\
\hline च & 98 & 425.3103 & 848.6060 \\
\hline v & $\underline{99}$ & 425.3109 & 848.6072 \\
\hline च & $1 \overline{00}$ & 426.2464 & 850.4783 \\
\hline v & 101 & 428.2618 & 854.5090 \\
\hline จ & 102 & 428.2618 & 854.5090 \\
\hline v & $\overline{103}$ & 428.2619 & 854.5092 \\
\hline v & 104 & 428.2622 & 854.5098 \\
\hline$\nabla$ & $\overline{105}$ & 428.2622 & 854.5099 \\
\hline v & 106 & 428.2629 & 854.5113 \\
\hline च & 107 & 428.2638 & 854.5130 \\
\hline v & 108 & 428.7651 & 855.5157 \\
\hline च & $\underline{109}$ & 428.7654 & 855.5163 \\
\hline च & 110 & 428.7657 & 855.5169 \\
\hline$\nabla$ & 111 & 428.7665 & 855.5185 \\
\hline$\nabla$ & $\underline{112}$ & 433.7326 & 865.4505 \\
\hline v & 113 & 435.2474 & 868.4802 \\
\hline$\nabla$ & $\overline{114}$ & 435.7546 & 869.4947 \\
\hline v & 116 & 435.7557 & 869.4968 \\
\hline v & 117 & 435.7561 & 869.4977 \\
\hline v & 118 & 435.7670 & 869.5193 \\
\hline v & $\underline{119}$ & 435.7674 & 869.5202 \\
\hline v & 120 & 435.7707 & 869.5269 \\
\hline च & $\overline{121}$ & 435.7722 & 869.5299 \\
\hline च & $\underline{122}$ & 435.7727 & 869.5308 \\
\hline च & $\underline{123}$ & 436.7742 & 871.5339 \\
\hline च & $\underline{124}$ & 436.7743 & 871.5340 \\
\hline च & $\underline{125}$ & 437.2904 & 872.5662 \\
\hline च & $\underline{126}$ & 437.7516 & 873.4887 \\
\hline च & 127 & 440.1126 & 878.2106 \\
\hline$\nabla$ & $\underline{128}$ & 440.1141 & 878.2135 \\
\hline च & $\underline{129}$ & 442.0916 & 882.1686 \\
\hline v & 130 & 442.7625 & 883.5104 \\
\hline D & 132 & 442.7640 & 883.5134 \\
\hline$\nabla$ & 133 & 444.1069 & 886.1993 \\
\hline v & 134 & 445.2882 & 888.5619 \\
\hline$\nabla$ & $\overline{135}$ & 448.2127 & 894.4108 \\
\hline च & 136 & 448.2136 & 894.4126 \\
\hline च & $\underline{137}$ & 448.2144 & 894.4143 \\
\hline จ & 138 & 449.7711 & 897.5276 \\
\hline v & 139 & 449.7714 & 897.5282 \\
\hline
\end{tabular}

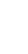

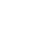

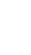

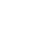

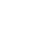

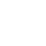

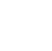

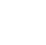

.

.

.

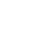


$\begin{array}{ll}140 & 449.7717\end{array}$

897.5287

898.5362

898.5371

898.5410

898.5799

898.5850

899.5651

900. 5154

900.5207

904.1309

904.2397

905.5447

905.5467

905.5478

905.5485

905. 5495

905.5500

905.5505

905.5505

905.5515

905.5517

905.5524

905.5525

905.5526

905.5528

905.5530

905.5530

905.5532

905.5533

905.5535

905.5543

905. 5544

905.5544

905.5545

905.5546

905.5547

905.5548

905.5551

905.5555

905. 5574

910. 2002

915.5613

915.5624

918.1722

921.4797

922.4093

922.4160

922.5145

926.4925

930.3265

934.3952

941.5677

941.5697

941.5723

941.5727

941.5729

941.5750

941.5750

941.5768

941.5779

941.5784

941.5789

942.5637

942.5642

950.4894

956.5369

956.5625

956.5642

959. 5889

960.2588

960.2720

961.2560

966.3470

970.5600

970.5813

972.5334

978.3425

986.5882

986.5898

988.5702

$\begin{array}{lll}\underline{230} & 495.2927 & 988.5709\end{array}$

$231 \quad 500.9528 \quad 999.8911$

$\begin{array}{lll}232 & 501.7951 & 1001.5756\end{array}$

$\begin{array}{lll}233 & 502.8147 & 1003.6149\end{array}$

$\underline{234} \quad 505.2692 \quad 1008.5238$

$\begin{array}{lll}235 & 505.2707 & 1008.5268\end{array}$

$\begin{array}{lll}236 & 511.3268 & 1020.6390\end{array}$

$\begin{array}{lll}237 & 513.2397 & 1024.4648\end{array}$

$\begin{array}{lll}\underline{238} & 515.2991 & 1028.5836\end{array}$

\begin{tabular}{l}
$239 \quad 515.7415 \quad 1029.4685$ \\
\hline
\end{tabular}

$240 \quad 515.7428 \quad 1029.4710$

$\overline{244} \quad 516.3145 \quad 1030.6144$

ป $\underline{\underline{245}} \quad 516.3156 \quad 1030.6167$

v $\underline{\underline{246}} \quad 516.8274 \quad 1031.6402$ 


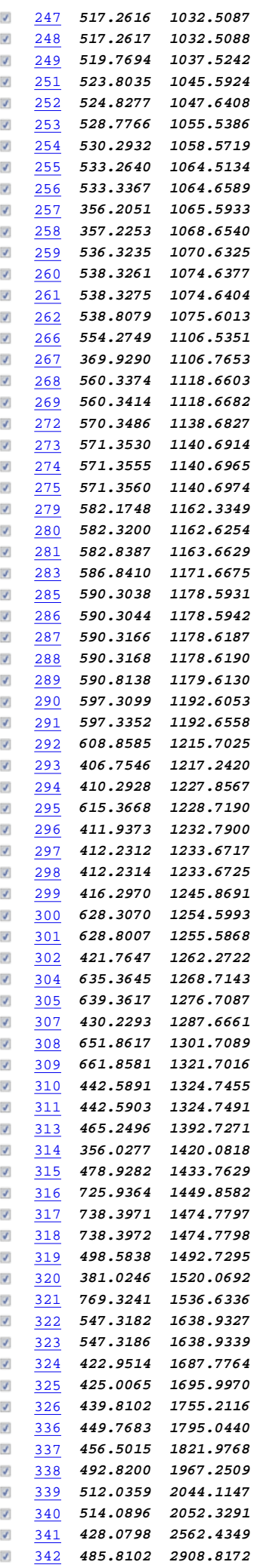

Search Parameters

Enzyme : Trypsin

variable modifications : Carbamidomethyl (C), oxidation (M) Mass values Monoisotopic

$\begin{array}{ll}\text { Protein Mass } & : \text { Unrestrictec } \\ \text { Peptide Mass Tolerance }: \pm 50 \mathrm{ppm}\end{array}$

Fragment Mass Tolerance: $\pm 0.6 \mathrm{Da}$

Max Missed Cleavages : Instrument type $\quad$ ESI-QUAD-TOF 


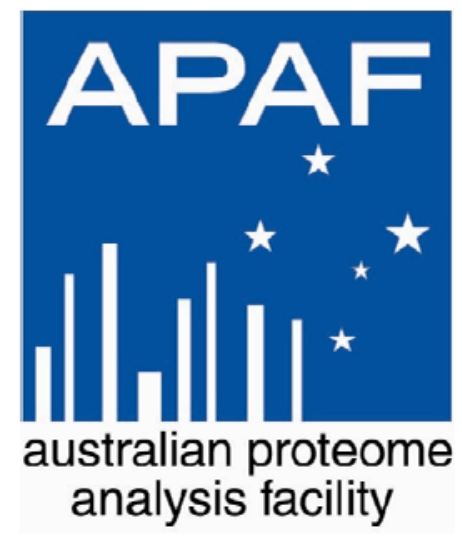

\section{Report: \\ ID nanoLC ESI MS/ MS}

Project code:

PROJ 17598

Client:

Sandi Dempsey

Mesynthes Ltd.

Victoria University of Wellington

Kelburn Parade, Kelburn

Wellington

New Zealand

Contact tel:

+6421 1548088

E-mail:

sandi.dempsey@vuw.ac.nz

Report date:

$15^{\text {th }}$ April 2015

Report prepared by: Matthew Fitzhenry

E-mail:

mfitzhenry@proteome.org.au

APAF - Australia's premier proteomics provider since 1995 
(a) Date samples received:

$31^{\text {st }}$ March 2015

(b) Sample names:

SD10.96.1 and SD10.96.2

\section{(c) I nstruments used}

Mass spectrometer: Triple TOF 5600 (AB Sciex)

NanoLC system: Eksigent Ultra nanoLC system (Eksigent)

Analytical column: Halo C18, $160 \AA, 2.7 \mu \mathrm{m}, 75 \mu \mathrm{m} \times 10 \mathrm{~cm}$

\section{(d) Sample preparation}

Samples S0.10.97.1 and S0.10.97.2 were reduced with $10 \mathrm{mM}$ DTT then alkylated with $25 \mathrm{mM}$ IAA. Sample S0.10.97.1 was digested overnight with $1 \mu \mathrm{g}$ trypsin, Sample S0.10.97.2 was digested overnight with $4 \mu \mathrm{g} \mathrm{GluC}$.

\section{(e) NanoLC ESI MS/ MS data acquisition}

$10 \mu \mathrm{L}$ of sample was injected onto a peptide trap (Michrome peptide Captrap) for pre-concentration and desalted with $0.1 \%$ formic acid, $2 \%$ $\mathrm{ACN}$, at $10 \mu \mathrm{L} / \mathrm{min}$ for 5 minutes. The peptide trap was then switched into line with the analytical column. Peptides were eluted from the column using a linear solvent gradient, with steps, from mobile phase A: mobile phase $B(95: 5)$ to mobile phase $A$ :mobile phase $B(60: 40)$ where mobile phase $A$ is $0.1 \%$ formic acid and mobile phase $B$ is $99.9 \% A C N / 0.1 \%$ formic acid at $550 \mathrm{~nL} / \mathrm{min}$ over a 42 min period. After peptide elution, the column was cleaned with $95 \%$ buffer B for 10 minutes and then equilibrated with buffer $A$ for 25 minutes before next sample injection.

The reverse phase nanoLC eluent was subject to positive ion nanoflow electrospray analysis in an information dependant acquisition mode (IDA). In IDA mode a TOFMS survey scan was acquired (m/z $350-1500,0.25$ 
second), with the ten most intense multiply charged ions (counts $>150$ ) in the survey scan sequentially subjected to MS/MS analysis. MS/MS spectra were accumulated for 200 milliseconds in the mass range m/z $100-1500$ with the total cycle time 2.3 seconds.

\section{(f) Data processing}

The raw data files (.wiff) were converted to mascot generic files (.mgf) using AB SCIEX CommandDriver software. They were submitted to Mascot (Matrix Science, UK) and searched against Swissprot database (Ovis aries [sp_sheep_140625] and All metazoa/animals)

\section{(g) Results}

Sequence coverage of Decorin using trypsin and Glu-C is:

$\begin{array}{llllll}10 & 20 & 30 & 40 & 50 & 60\end{array}$

MKATIIFFLV AQVSWAGPFQ QKGLFDFMLE DEASGIGPEE RFHEVPELEP MGPVCPFRCQ

$\begin{array}{llllll}70 & 80 & 90 & 100 & 110 & 120\end{array}$

CHLRVVQCSD LGLEKVPKDL PPDTALLDLQ NNKITEIKDG DFKNLKNLHT LILINNKISK

$\begin{array}{llllll}130 & 140 & 150 & 160 & 170 & 180\end{array}$

ISPGAFAPLV KLERLYLSKN QLKELPEKMP KTLQELRVHE NEITKVRKSV FNGLNQMIVV

$\begin{array}{llllll}190 & 200 & 210 & 220 & 230 & 240\end{array}$

ELGTNPLKSS GIENGAFQGM KKLSYIRIAD TNITTIPQGL PPSLTELHLD GNKITKVDAA

$\begin{array}{llllll}250 & 260 & 270 & 280 & 290 & 300\end{array}$

SLKGLNNLAK LGLSFNSISA VDNGSLANTP HLRELHLNNN KLVKVPGGLA DHKYIQVVYL

$\begin{array}{llllll}310 & 320 & 330 & 340 & 350 & 360\end{array}$

HNNNISAIGS NDFCPPGYNT KKASYSGVSL FSNPVQYWEI QPSTFRCVYV RAAVQLGNYK

This sequence coverage covers $24 \mathrm{kDa}$ of the Decorin protein.

The ESI analysis of the gel from the previous analysis suggests a number of proteins, including Decorin and Actin. The gel band observed contains all of 
these proteins. It is unknown if the $12 \mathrm{kDa}$ fragment found in the gel run in the previous result (PROJ 17432) belongs specifically to Decorin.

Owing to the large number of proteins identified, (electrospray) top-down analysis of Decorin is not possible. Running an intact mass by MALDI may find evidence of a $12 \mathrm{kDa}$ fragment.

The Mascot data can be examined by logging into the client access server (http://bio.proteome.org.au/mascot/cgi/login. pl?referer=../x-cgi/msreview.exe). Username is your email address, and the password is vicuniwell.

\begin{tabular}{|l|l|l|l|}
\hline Sample Name & Input Parameters & $\begin{array}{l}\text { Search } \\
\text { Number }\end{array}$ & Database \\
\hline SD01.10.97.1 & Trypsin & 90211 & sp_sheep_140625 \\
\hline SD01.10.97.2 & Glu-C (E) & 90238 & sp_sheep_140625 \\
\hline SD01.10.97.1 & Trypsin & 90298 & All animal species \\
\hline SD01.10.97.2 & Glu-C (E) & 90299 & All animal species \\
\hline $\begin{array}{l}\text { *150213_P17432 } \\
\text { SD1096-2_1 }\end{array}$ & Trypsin & 89674 & sp_sheep_140625 \\
\hline
\end{tabular}

*ESI result of $12 \mathrm{kDa}$ gel piece from PROJ 17432

\section{Acknowledgment}

Any publication arise from this work should be acknowledged the contribution of APAF with the words "This work was undertaken at APAF the infrastructure provided by the Australian Government through the National Collaborative Research I nfrastructure Strategy (NCRIS)."

We welcome your feedback on our services. To go into our quarterly draw to win a prepaid $\$ 200$ debit card follow the link and complete a 5 min questionaire.

https://mqedu.qualtrics.com/SE/?SID=SV_3yIN4UQfHDq0FmZ 


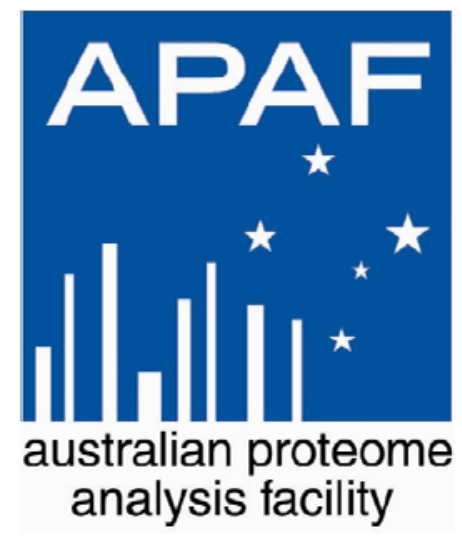

\section{Report: \\ ID nanoLC ESI MS/ MS}

Project code:

PROJ 17906

Client:

Sandi Dempsey

Mesynthes Ltd.

Victoria University of Wellington

Kelburn Parade, Kelburn

Wellington

New Zealand

Contact tel:

+64 211548088

E-mail:

sandi.dempsey@vuw.ac.nz

Report date:

$21^{\text {st }}$ July 2015

Report prepared by: Matthew Fitzhenry

E-mail:

mfitzhenry@proteome.org.au

\section{APAF - Australia's premier proteomics provider since 1995}


(a) Date samples received:

$16^{\text {th }}$ June 2015

(b) Sample names:

SD97.1 and SD97.2

(c) Instruments used

Mass spectrometer: Triple TOF 5600 (AB Sciex)

NanoLC system: Eksigent Ultra nanoLC system (Eksigent)

Analytical column: Halo C18, $160 \AA, 2.7 \mu \mathrm{m}, 75 \mu \mathrm{m} \times 10 \mathrm{~cm}$

\section{(d) Sample preparation}

Sample was received from APAF Protein Chemistry, treated by sizeexclusion chromatography, fractionated into a 96 well plate. Mobile phase was $50 \mathrm{mM}$ sodium phosphate $(\mathrm{pH}$ 7) $150 \mathrm{mM} \mathrm{NaCl}$. From this plate, fractions $A 1$ to $A 12$ were combined $(60 \mu \mathrm{L})$ to give sample subsequently labelled "SD97-1". Fractions B8 to B1 (reverse order) and Fractions C1 to C12 were combined ( $60 \mu \mathrm{L}$ ) to give sample subsequently labelled "SD972 ". These combined fractions correspond to time points in SEC gradient 20-26 min and 28-38 min, respectively.

SD97-1 (720 $\mu \mathrm{L})$ and SD97-2 (1200 $\mu \mathrm{L})$ were reduced with a final concentration of $10 \mathrm{mM}$ DTT at $60^{\circ} \mathrm{C}$ for 1 hour. The samples were alkylated with a final concentration of $25 \mathrm{mM}$ IAA for 30 min in dark at room temperature. $500 \mathrm{ng}$ of trypsin was added to each sample, digested overnight at $37^{\circ} \mathrm{C}$. Each sample was passed through a conditioned OMIX C18 $100 \mu \mathrm{L}$ tip (Agilent/Varian, A57003100K) to concentrate and desalt, prior to eluting in $100 \mu \mathrm{L}$ acetonitrile/formic acid. The sample was dried, then reconstituted in LC loading buffer prior to analysis.

To confirm the result, the two samples were re-run $(40 \mu \mathrm{L} \& 20 \mu \mathrm{L}$ loadings for SD97-1 \& SD97-2, respectively) with blanks in between. 


\section{(e) NanoLC ESI MS/ MS data acquisition}

Sample $(10,20$ or $40 \mu \mathrm{L}$ ) was injected onto a peptide trap (Michrome peptide Captrap) for pre-concentration and desalted with $0.1 \%$ formic acid, $2 \% \mathrm{ACN}$, at $10 \mu \mathrm{L} / \mathrm{min}$ for 5 minutes. The peptide trap was then switched into line with the analytical column. Peptides were eluted from the column using a linear solvent gradient, with steps, from $\mathrm{H}_{2} \mathrm{O}: \mathrm{CH}_{3} \mathrm{CN}(95: 5 ;+0.1 \%$ formic acid) to $\mathrm{H}_{2} \mathrm{O}: \mathrm{CH}_{3} \mathrm{CN}(5: 95 ;+0.1 \%$ formic acid) with constant flow $(500 \mathrm{~nL} / \mathrm{min})$ over an 80 min period. The LC eluent was subject to positive ion nanoflow electrospray MS analysis in an information dependant acquisition mode (IDA).

In the IDA mode a TOFMS survey scan was acquired ( $\mathrm{m} / \mathrm{z} 350-1500,0.25$ seconds), with ten largest multiply charged ions (counts $>150$ ) in the survey scan sequentially subjected to MS/MS analysis. MS/MS spectra were accumulated for 200 milli-seconds (m/z 100-1500) with rolling collision energy.

\section{(f) Data processing}

The raw data files (.wiff) were converted to mascot generic files (.mgf) using AB SCIEX CommandDriver software. They were submitted to Mascot (Matrix Science, UK) and searched against Swissprot database (Ovis aries) and (Mus musculus).

Wiff files were also searched against SwissProt (Ovis aries) using ProteinPilot v4.2. The database contains 12832 proteins.

Ovis aries searches

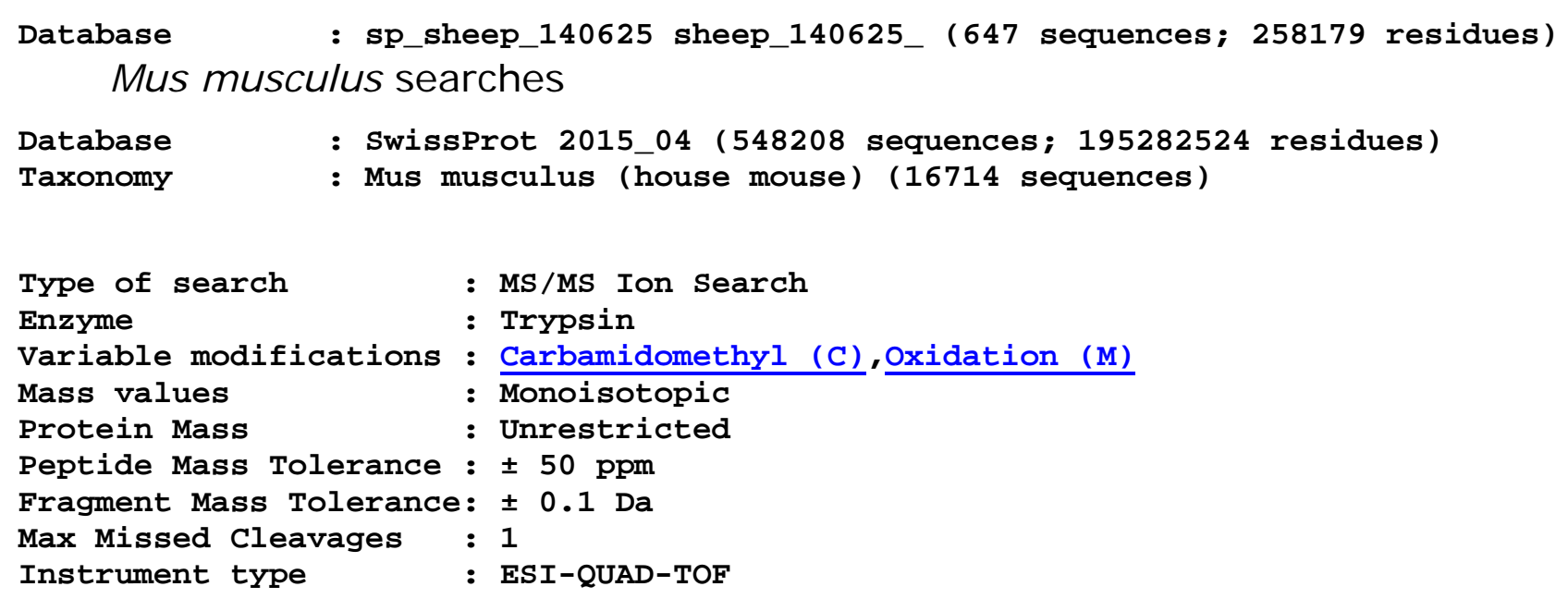


The presence of albumin carry-over was present in blank runs (seen in Mouse searches) prior to each sample. This is shown by including the prior blank Mascot searches.

\section{Acknowledgment}

Any publication arise from this work should be acknowledged the contribution of APAF with the words "This work was undertaken at APAF the infrastructure provided by the Australian Government through the National Collaborative Research I nfrastructure Strategy (NCRIS)."

We welcome your feedback on our services. To go into our quarterly draw to win a prepaid $\$ 200$ debit card follow the link and complete a 5 min questionaire.

https://mqedu.qualtrics.com/SE/?SID=SV_3ylN4UQfHDq0FmZ 
Appendix 5: A gradient based chemotaxis assay for cell motility 


\section{A gradient based chemotaxis assay for cell 2 motility}

$3 \quad$ Sandi G. Dempsey ${ }^{1,2}$ sandi.dempsey@vuw.ac.nz

4 Barnaby C. H. May ${ }^{1}$ barnaby.may@aroabio.com

$5 \quad$ Darren J. Day ${ }^{2}$ darren.day@vuw.ac.nz (corresponding author)

$6 \quad{ }^{1}$ Aroa Biosurgery, 2 Kingsford Smith Place, Auckland, 2022, New Zealand.

$7{ }^{2}$ School of Biological Sciences, Victoria University of Wellington, Kelburn Parade 2016, 8 Wellington, New Zealand. 


\section{Abstract}

\section{Background}

1 Cell motility is critical for morphogenesis, wound healing and homeostasis in living

2 organisms and different assays have been developed to model the process. We describe a

3 simple and cost effective assay to measure the chemotactic response of different adherent

4 cells in vitro. The point of difference in this assay is the use of a central agarose plug to

5 establish an immobilised chemical gradient along which cells migrate. This method is

6 compared to the established "scratch" assay.

\section{Results}

7 Adherent mammalian cell lines respond to growth factors and cell conditioned media in a

8 dose dependent manner using this assay in the same way that they react in a scratch assay.

\section{Conclusion}

9 Using this approach, cells migrate in response to a chemotactic gradient. This method is

10 unique in that a gradient is established without the need for modified cell culture apparatus,

11 chambers or membranes. This assay requires small volumes of test materials and can be used

12 for high throughput screening applications.

\section{Keywords}

13 Agarose plug, chemotaxis, motility, scratch assay, fibroblast, HUVEC, mesenchymal stem

14 cell, chemokine gradient.

A gradient based chemotaxis assay for cell motility 


\section{Background}

1 The control of mammalian cell migration through living tissue is crucial for processes such as

2 embryonic development, leukocyte migration, wound healing, tumour invasion, and

3 metastasis. Early studies of cell migration focused on the recruitment of leukocytes during

4 infection and inflammation[1,2]. More recently, studies in cell motility during angiogenesis,

5 tumour growth and wound healing have grown in prominence[3-6]. A number of in vitro

6 models determine the chemotactic potential of a solution, these can be described as

7 chemokinetic, phagokinetic, chemotactic or invasion assays, based on their design. These

8 models do not represent the complex dynamics of living tissue, but they provide a

9 quantitative means of assessing cell motility and recruitment.

10 Chemokinetic methods, such as the "scratch" assay, measure a change in the level of cell

11 motility in response to a solution[7]. Scratch assays are often used with endothelial progenitor

12 cells to demonstrate the pro- or anti-angiogenic properties of a compound. In this type of

13 assay, a solution is incubated with an interrupted monolayer of adherent cells and the margin

14 of the stratum is measured at various time points to determine the movement of cells to

15 reform the monolayer.

16 Phagokinetic assays use time lapse photography and/or micro carrier beads to track

17 individual cells, a number of variables such as rate and direction are employed to demonstrate

18 cell motility[8-10]. While these assays will demonstrate changes in cell motility, they do not

19 model a chemotactic concentration gradient, which cells use for directional migration

20 response in living tissue.

21 Chemotactic assays demonstrate cell migration across a cell permeable barrier, such as the

22 Boyden or Transwell® chamber, towards a chemical stimulus[11]. In this type of assay,

A gradient based chemotaxis assay for cell motility

Feb-16 
1 adjacent chambers establish a difference in chemokine concentration, which models a

2 chemotactic chemical gradient.

3 Invasion assays employ basement membrane proteins to model the three dimensional

4 extracellular matrix environment and demonstrate a cells ability to permeate the matrix. This

5 models more complex systems where cells are required to penetrate surrounding proteins in

6 order to migrate towards a chemokine. These are often used in studies of angiogenesis,

7 tumour metastasis and to screen for anti-migratory compounds[5, 12-14].

8 Here we describe a method to generate a chemotactic gradient that can be used to screen for

9 test articles that stimulate cell migration. A monolayer of adherent cells is established using

10 standard cell culture micro titre plate, then a layer of molten low melt agarose is allowed to

11 set over the cells. A central area of the agarose is removed using a biopsy punch and a small

12 volume of agarose; the central "plug", containing a chemotactic solution is added to the

13 centre. Migrated cells underneath the plug can be counted or imaged in real time or at an end

14 point. Unlike the system generated in a Boyden chamber, the chemical gradient is

15 immobilised in an agarose plug to generate a change in the chemical concentration across

16 each well. The method is conducted in a 96 well plate and requires very low volumes of

17 control and test solutions. In addition, the cost of this assay is significantly lower than

18 existing chemotactic assays as this method does not require a specialist membrane.

\section{Results and discussion}

19 In this assay a chemical gradient within a layer of agarose above a cell monolayer. This was

20 demonstrated with blue dyes of high, mid and low molecular weights which were added to

21 the central "plug" to demonstrate the gradient across the well; blue dextran (Mw: 2,000,000

$22 \mathrm{gmol}^{-1}$ ), trypan blue (Mw: $872 \mathrm{gmol}^{-1}$ ) and toluidine blue (Mw: $240 \mathrm{gmol}^{-1}$ ). Images taken at
A gradient based chemotaxis assay for cell motility
Feb-16 
1 hourly intervals demonstrated a high concentration of each dye at the centre of each well,

2 which diffuses over time. As shown in Figure 1, the extent of diffusion depended on the size

3 of the dye, with blue dextran (top row) diffusing slower than trypan blue (centre row) and

4 toluidine blue (bottom row).

$5 \quad$ [Figure 1]

6 Three cell lines, mesenchymal stem cells (MSC), dermal fibroblasts (D551) and human

7 umbilical vein endothelial cells (HUVEC), migrated from the edges of the well when a

8 chemotactic gradient was established using an agarose plug. The effect was dose dependent,

9 such that higher concentrations of chemo-attractant caused more cells to migrate into the

10 central area. Figure 2 shows representative images of mesenchymal stem cells (MSC) that

11 have migrated to the central plug area in response to $0,2.5$ and $10 \mathrm{ng} / \mathrm{mL}$ of PDGF (A, B and

12 C respectively) that was present in the central plug of the agarose.

13 [Figure 2]

14 In order to demonstrate that this increase in cell number was due to the movement of cells

15 towards the central plug, and not a global increase in cell population, cells were counted

16 inside and outside the central well. Figure 3 shows that while cell number within the central

17 well area changed significantly in response to PDGF, the number of cells in an area of the

18 same size outside the central plug did not.

19 [Figure 3]

20 Three cell lines responded to the presence of recombinant growth factors in our assay. As

21 shown in Figure 4, MSCs (A) and D551s (B) migrated towards higher concentrations of

22 PDGF, while HUVECs (C) migrated towards a higher concentration of VEGF in the agarose

23 plug assay. For comparison, a conventional scratch assay was performed with the same cell

24 lines and growth factors (D, E and F respectively). As expected, increasing the concentration A gradient based chemotaxis assay for cell motility

Feb-16 
1 of chemotactic growth factor increased the cell count in the agarose plug assay and the

2 percentage closure of the scratch. Results from both assays correlated well, with MSCs and

3 D551 fibroblasts cells responding to PDGF and HUVEC responding to VEGF in a dose

4 dependent manner. This data is compiled from six replicates (wells). The results of the

5 agarose plug assay are consistent with the results of the established scratch assay with regard

6 to known growth factors[13-17]. It should be noted that the scratch assay was not as effective

7 on D551 as other lines, perhaps because these cells are highly migratory the media only

8 controls show a high level of "scratch closure" and the baseline in this experiment is higher

9 compared with other cells (shown in Figure 4 E). The scratch assay may not be an

10 appropriate way to test motility in this particular cell line, under these conditions.

11 [Figure 4]

12 In Figure 4, a significant change in motility is recorded for each assay. The plug assay uses

13 only $10 \mu \mathrm{L}$ of each sample, and the lowest concentration of PDGF to achieve a significant

14 change in cell number compared with the media control is $5 \mathrm{ng} / \mathrm{mL}$, or $0.05 \mathrm{ng}$ per well.

15 Using the scratch assay, $5 \mathrm{ng} / \mathrm{mL}$ is also the lowest concentration to achieve a significant

16 result, however this assay requires $100 \mu \mathrm{L}$ of sample so the total amount of growth factor

17 required per well is $0.5 \mathrm{ng}$. For HUVEC the lower limits are the same for both plug and

18 scratch assays.

19 To demonstrate the assay's versatility, the migration of MSCs towards mouse embryonic

20 fibroblast (3T3) conditioned media was demonstrated. Figure 5 shows dose dependent MSC

21 migration towards the central plug that contains conditioned media diluted in normal media

$22(\mathrm{v} / \mathrm{v})$.

23 [Figure 5]

A gradient based chemotaxis assay for cell motility

Feb-16 
1 The data presented above shows that the agarose plug assay can be used to detect a chemo

2 attractant in a dose dependent manner, in media supplemented with a recombinant growth

3 factor or in a mixture of proteins such as those found in cell conditioned media.

4 Cell migration is a fundamental biological process and different cell types employ

5 mechanisms in response to chemical and physical stimuli to achieve this[18]. Chemical

6 stimuli can be chemokinetic, an increase in random movement; chemotactic, a directed

7 response to a gradient of a soluble compound; and haptotactic, a directed response to surface

8 gradient. The type of assay employed in a study on cell motility should reflect the nature of

9 cell migration in the system being modelled.

10 The "scratch" assay is a widely used model, which fundamentally demonstrates that cells

11 grown in close contact with each other will respond to a disruption of cell-cell contact by

12 closing that gap. Endothelial cells, for instance, achieve this by migration and proliferation,

13 as they would during the formation of new vessels during angiogenesis in injury or tumour

14 growth. Inferences can be made regarding pro- or anti- angiogenic properties of compounds

15 or chemicals that alter this process. The fundamental disadvantage of this method is that cell

16 motility is recorded, without reference to directional chemotaxis. Endothelial cells can move

17 individually in a 'random walk' but also in response to VEGF in a chemotactic manner[7].

18 Cells are able to sense the direction of the VEGF gradient via VEGF receptors around the cell

19 that induce polarity by actin polymerisation. During angiogenesis, endothelial cells respond

20 to a number of cues, so a scratch assay is suitable way to screen for inhibitors of cell

21 migration, because an effective inhibitor will prevent scratch closure, regardless of the type

22 of cell motility.

23 During wound healing, different methods of motility are employed by different cell types

24 such as circulating leukocytes, bone marrow derived mesenchymal stem cells or fibroblasts 
1 and keratinocytes from neighbouring tissue[19]. These cells respond to a number of signals,

2 including surface bound and soluble chemical gradients, direct current electric fields and

3 surface rigidity[20]. Assays that employ a soluble chemical gradient may be a more

4 appropriate model for some cells, as a directional sensitivity to the compound is required.

5 This agarose plug assay offers several advantages when compared with other cell migration

6 assays. Firstly, the plug assay establishes a chemical gradient from the central well to the

7 surrounding agarose plug, making this a chemotactic assay, compared with the scratch assay

8 which detects chemokinetic activity without regard to the direction of a chemical gradient[21]

9 Secondly, compared with chamber-based assays, the procedure is inexpensive as no specialist

10 chambers or membranes are required. Using an agarose plug generates a chemical gradient

11 and the rate of diffusion can be controlled by modifying the concentration of agarose used

12 inside the central plug, making this a versatile alternative to chamber based methods. Plates

13 can be set up in a 96 well plate, allowing large numbers of samples to be tested at once.

14 Thirdly, the quantity of each test solution required to create the agarose plug is minimal

15 compared with both Boyden chamber and scratch methods. A $10 \mu \mathrm{L}$ central well is sufficient

16 to induce cell migration in a 96 well plate set up, compared with over $100 \mu \mathrm{L}$ in the scratch

17 assay or Boyden chamber assay.

\section{Conclusion}

18 This simple and cost effective method is an effective tool for screening potential mediators of

19 cell motility. The assay provides advantages over well-established scratch and membrane

20 invasion assay due to the differential gradient of a test solution established between the 
1 agarose plug and central well. This type of assay is valuable for in vitro studies in cell

2 migration and recruitment.

\section{Methods}

\section{General}

3 The blue dyes trypan blue, dextran blue and toluidine blue (Sigma Aldrich) were dissolved in

$4 \mathrm{ddH}_{2} \mathrm{O}$. Human dermal fibroblasts cells (D551) were obtained from ATCC (CCL-110) and

5 cultured in DMEM media (Life Technologies, USA) supplemented with $100 \mathrm{U} / \mathrm{mL}$ penicillin

6 and $100 \mu \mathrm{g} / \mathrm{mL}$ streptomycin (Life Technologies) with 10\% fetal calf serum (Life

7 Technologies).

8 Human mesenchymal stem cells (MSCs) were isolated from haemangioma tissue at Victoria

9 University of Wellington (Wellington, NZ), as previously described[22]. Isolated human

10 umbilical vein endothelial cells (HUVEC) were provided by Otago University (Christchurch,

$11 \mathrm{NZ}$ ) and cultured in RPMI media (Life Technologies) supplemented with $50 \mu \mathrm{g} / \mathrm{mL}$

12 endothelial cell growth supplemented (ECGS) (Sigma Aldrich) with $100 \mathrm{U} / \mathrm{mL}$ penicillin and

$13100 \mu \mathrm{g} / \mathrm{mL}$ streptomycin with $10 \%$ fetal calf serum. Culture flasks and plates were treated

14 with $0.5 \%$ gelatine for 1 hour prior to seeding HUVEC cells. Cells were cultured in a

15 humidified incubator at $37{ }^{\circ} \mathrm{C}$ with $5 \% \mathrm{CO}_{2}$.

16 Platelet derived growth factor (PDGF) (PeproTech, USA) was used as a chemo attractant for

17 D551 fibroblast cells and MSC. The growth factor was diluted in DMEM supplemented with

$181 \%$ fetal calf serum at a range of $0-25 \mathrm{ng} / \mathrm{mL}$. Vascular Endothelial Growth Factor (VEGF)

19 (BD Sciences, USA) was diluted in RPMI supplemented with $50 \mu \mathrm{g} / \mathrm{mL}$ ECGS, with $1 \%$

20 foetal calf serum at a range of $0-25 \mathrm{ng} / \mathrm{mL}$ as a chemo attractant for HUVEC cells.

A gradient based chemotaxis assay for cell motility

Feb-16 
1 Conditioned media was prepared by culturing 3T3 mouse embryonic fibroblast cells (ATCC

2 CRL-1658) for 24 hours then harvesting the media. The conditioned media was sterilized

3 through a $0.22 \mu \mathrm{m}$ filter (BioFil, China) prior to use. Conditioned media was further diluted

4 to $1 \%, 5 \%, 10 \%$ and $50 \%(\mathrm{v} / \mathrm{v})$ with normal DMEM.

5 Neutral red stain (Sigma Aldrich) was prepared as a $0.008 \%$ solution in cell culture media

6 containing $25 \mathrm{mM}$ HEPES buffer (Sigma Aldrich).

\section{Agarose Plug Migration Assay}

7 Cells were seeded onto 96 well plates at a density of 10,000 cells per well and incubated for

824 hours (until 80\% confluent). Low melting temperature agarose (Nuseive ${ }^{\circledR}$ GTG ${ }^{\circledR}$,

9 Cambrex, USA) was added to sterile phosphate buffered saline and microwaved in short

10 bursts to give a molten 3\% agarose solution (w/v). Molten agarose was mixed with an equal

11 volume of growth media with $2 \%$ fetal calf serum so that a final concentration of $1.5 \%(\mathrm{w} / \mathrm{v})$

12 agarose and $1 \%$ fetal calf serum was achieved. The mixture was prepared immediately prior

13 to use and maintained in a molten state at $40{ }^{\circ} \mathrm{C}$ in a water bath.

14 Media was gently removed from cells and $100 \mu \mathrm{L}$ of the molten agarose plus media mixture

15 was added to each well and allowed to set at room temperature for 20 minutes. Holes to form

16 the central plug were made in each well using a $2 \mathrm{~mm}$ diameter biopsy punch (HealthLink ${ }^{\circledR}$,

17 USA). A pipette tip attached to a vacuum line was used to remove the circular section of

18 agarose and underlying cells from the middle of each well. Media $(50 \mu \mathrm{L})$ was added to each

19 well and then removed using the vacuum line to ensure all cells were removed from the

20 central "plug" area.

21 A fresh aliquot of molten agarose and growth media was prepared containing an appropriate

22 concentration of growth factor, conditioned media of dye. The central hole was filled with 10 
$1 \mu \mathrm{L}$ of the molten solution using a pre warmed pipette tip. When set, $50 \mu \mathrm{L}$ of media was

2 added to the cover the agarose and plates were incubated for eight to 12 hours (depending on

3 cell type). The incubation period was optimized for each cell line. D551 fibroblasts, which

4 migrated rapidly into the central space could be counted after eight hours incubation, while

5 HUVEC and MSC took longer requiring 12 hours.

6 To facilitate visualisation of viable cells, wells were stained by adding $50 \mu \mathrm{L}$ of neutral red

$7 \quad(0.008 \% \mathrm{w} / \mathrm{v})$ for 3 hours. Cells within the central plug were imaged using a DP12 camera

8 (Olympus, Japan) attached to an inverted CKX41 microscope (Olympus, Japan) and counted

9 using "cell counter" plug in for ImageJ (NIH, USA).

\section{Scratch Test}

10 A horizontal line was drawn across the centre of each well on a 96 well plate before cells

11 were seeded at a density of 20,000 cells per well and incubated for 24 hours (until $90 \%$

12 confluent). A scratch was made in the monolayer with a pipette tip, perpendicular to a line

13 drawn on the underside of the plate. Dislodged cells were removed by gentle washing with

14 cell culture media. Media $(100 \mu \mathrm{L})$ containing a dilution series of known chemo-attractants,

15 PDGF (for D551 and MSC) or VEGF (for HUVEC), was then added to the scratched

16 monolayer. Each well was imaged using a DP12 camera (Olympus, Japan) attached to an

17 inverted CKX41 microscope (Olympus, Japan) before and after six (D551) or 12 hours

18 (HUVEC and MSC) incubation with growth factors, using the line drawn to orient the

19 position of the camera. The change in area of the scratch was expressed as a percentage of the

20 original scratch size using Image (NIH, USA) to determine the denuded area of the

21 monolayer.

A gradient based chemotaxis assay for cell motility

Feb-16 


\section{Statistical analysis}

1 Results for the cell count and scratch closure assays were the mean values and error bars

2 show the standard deviation from six replicates (wells). Significant values $(*)$, in relation to

3 the media control wells, were calculated by student's t-test $(\mathrm{p}<0.05)$.

\section{List of abbreviations used}

$4 \quad 3$ T3 embryonic fibroblast cell line

5 ATCC American Type Culture Collection

6 D551 dermal fibroblast

$7 \quad \mathrm{ddH}_{2} \mathrm{O}$ distilled water

8 DMEM Dulbecco's modified eagle medium

9 ECGS endothelial cell growth supplement

10 GF growth factor

11 HUVEC human umbilical vein endothelial cell

12 MSC mesenchymal stem cell

13 PDGF platelet derived growth factor

14 RPMI Rosewell Park Memorial Institute Medium

15 VEGF vascular endothelial growth factor 


\section{Competing interests}

1 BCHM is a shareholder of Aroa Biosurgery Ltd.

\section{Authors' contributions}

2 SGD carried out experimental procedures for the assay development and wrote the

3 manuscript

4 BCHM contributed to the development of method, analysis of data and revisions to

5 manuscript

6 DJD contributed to the conceptualization and development of methods, analysis of data and

7 revisions to manuscript

\section{Authors information}

8 SGD Aroa Biosurgery, 2 Kingsford Smith Place, Auckland, 2022, New Zealand and

9 School of Biological Sciences, Victoria University of Wellington, Kelburn Parade 2016,

10 Wellington, New Zealand

11 BCHM Aroa Biosurgery, 2 Kingsford Smith Place, Auckland, 2022, New Zealand

12 DJD School of Biological Sciences, Victoria University of Wellington, Kelburn Parade 2016,

13 Wellington, New Zealand

\section{Acknowledgements}


1 This work was supported by a scholarship from the Ministry of Science and Innovation, NZ (MSMA1003). We would also like to acknowledge the assistance of Dr Sarah Gunningham at the University of Otago (Christchurch, NZ) for providing the HUVEC cells, Dr Jun Jia at the Victoria University of Wellington (Wellington, NZ) for isolating the mesenchymal stem cells and Dr Ryan Steel for his critical review of the work.

\section{References}

6 1. Hallab N, Jacobs JJ, Black J: Hypersensitivity to metallic biomaterials: a review of

7 leukocyte migration inhibition assays. Biomaterials 2000, 21:1301-1314.

8 2. Radeke HH, Ludwig RJ, Boehncke W-H: Experimental approaches to lymphocyte

9 migration in dermatology in vitro and in vivo. Exp Dermatol 2005, 14:641-666.

10 3. Decaestecker C, Debeir O, Ham P, Kiss R: Can anti-migratory drugs be screened in 11 vitro? A review of 2D and 3D assays for the quantitative analysis of cell migration. Med 12 Res Rev 2007, 27:149-176.

13 4. Irvine SM, Cayzer J, Todd EM, Lun S, Floden EW, Negron L, Fisher JN, Dempsey SG, 14 Alexander A, Hill MC, O'Rouke A, Gunningham SP, Knight C, Davis PF, Ward BR, May 15 BC: Quantification of in vitro and in vivo angiogenesis stimulated by ovine forestomach 16 matrix biomaterial. Biomaterials 2011, 32:6351-6361.

17 5. Kramer N, Walzl A, Unger C, Rosner M, Krupitza G, Hengstschläger M, Dolznig H: In 18 vitro cell migration and invasion assays. Mutat Res 2013, 752:10-24.

19 6. Yi EH, Yoo H, Noh KH, Han S, Lee H, Lee J-K, Won C, Kim B-H, Kim M-H, Cho C-H, 20 Ye S: BST-2 is a potential activator of invasion and migration in tamoxifen-resistant 21 breast cancer cells. Biochem Biophys Res Commun 2013, 435:685-690.

7. Liang C-CC, Park AY, Guan J-LL: In vitro scratch assay: a convenient and inexpensive method for analysis of cell migration in vitro. Nat Protoc 2007, 2:329-333.

8. Debeir O, Camby I, Kiss R, Ham P, Decaestecker C: A model-based approach for automated in vitro cell tracking and chemotaxis analyses. Cytom Part J Int Soc Anal Cytol 2004, 60:29-40.

9. Niinaka Y, Haga A, Raz A: Quantification of cell motility : gold colloidal phagokinetic 28 track assay and wound healing assay. Methods Mol Med 2001, 58:55-60.

29 10. Nogalski MT, Chan GCT, Stevenson EV, Collins-McMillen DK, Yurochko AD: A 30 quantitative evaluation of cell migration by the phagokinetic track motility assay. $J$ Vis 31 Exp JoVE 2012:e4165.

A gradient based chemotaxis assay for cell motility 
1 11. Boyden S: The chemotactic effect of mixtures of antibody and antigen on

2 polymorphonuclear leucocytes. $J$ Exp Med 1962, 115:453-466.

3 12. Auerbach R, Lewis R, Shinners B, Kubai L, Akhtar N: Angiogenesis assays: a critical 4 overview. Clin Chem 2003, 49:32-40.

5 13. Seymour LW, Shoaibi MA, Martin A, Ahmed A, Elvin P, Kerr DJ, Wakelam MJ:

6 Vascular endothelial growth factor stimulates protein kinase $\mathrm{C}$-dependent

7 phospholipase D activity in endothelial cells. Lab Investig J Tech Methods Pathol 1996, 8 75:427-437.

9 14. Yoshida A, Anand-Apte B, Zetter BR: Differential endothelial migration and

10 proliferation to basic fibroblast growth factor and vascular endothelial growth factor.

11 Growth Factors Chur Switz 1996, 13:57-64.

12 15. Heldin $\mathrm{CH}$, Westermark B: Platelet-derived growth factors: a family of isoforms that 13 bind to two distinct receptors. Br Med Bull 1989, 45:453-464.

14 16. Higaki M, Sakaue H, Ogawa W, Kasuga M, Shimokado K: Phosphatidylinositol 315 kinase-independent signal transduction pathway for platelet-derived growth factor16 induced chemotaxis. J Biol Chem 1996, 271:29342-29346.

17 17. Kundra V, Anand-Apte B, Feig LA, Zetter BR: The chemotactic response to PDGF18 BB: evidence of a role for Ras. J Cell Biol 1995, 130:725-731.

19 18. Lauffenburger DA, Horwitz AF: Cell migration: a physically integrated molecular 20 process. Cell 1996, 84:359-369.

21 19. Barrientos S, Stojadinovic O, Golinko MS, Brem H, Marjana T-C: Growth factors and 22 cytokines in wound healing. Wound Repair Regen Off Publ Wound Heal Soc Eur Tissue 23 Repair Soc 2008, 16:585-601.

20. Czirok A: Endothelial cell motility, coordination and pattern formation during vasculogenesis. Wiley Interdiscip Rev Syst Biol Med 2013, 5:587-602. WS, Zigmond SH: A proposal for the definition of terms related to locomotion of leucocytes and other cells. Cell Biol Int Rep 1977, 1:391-397. 


\section{Illustrations and figures}

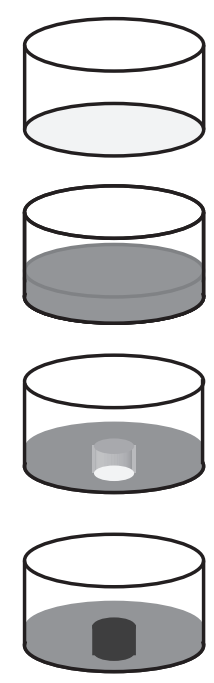

Monolayer of cells established

Molten agarose layer set up and allowed to set.

Central area of cells and agarose

removed with biopsy punch

Inner "plug" made of molten agarose mixed with sample, added to centre

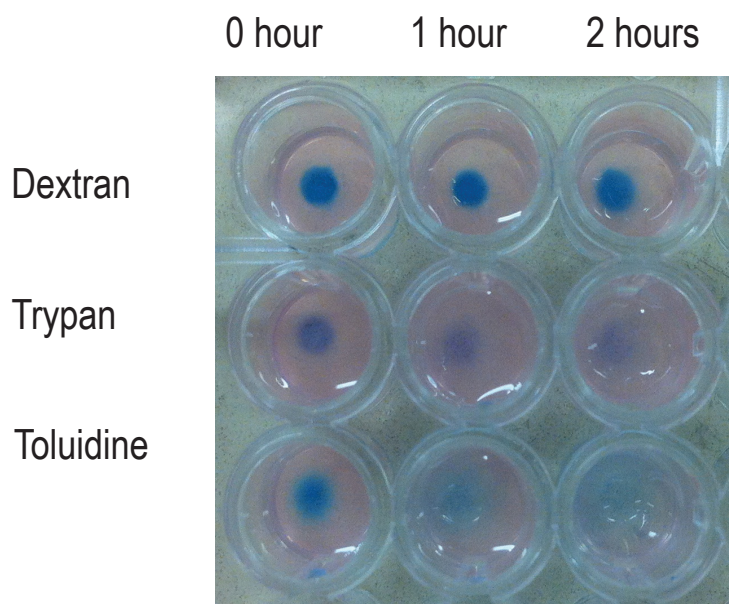

1 Figure 1: Establishing a chemical gradient in the agarose plug assay. A gradient is

2 generated within an agarose layer; samples in the central plug were labelled with blue dyes of

3 different molecular weights; blue dextran (top row), trypan blue (middle row) and toluidine

4 blue (bottom row). Images taken at hourly intervals (left to right columns) demonstrate that

5 the extent of dye diffusion depended on their molecular weight, with blue dextran diffusing

6 slower than trypan blue and toluidine blue.

A gradient based chemotaxis assay for cell motility 

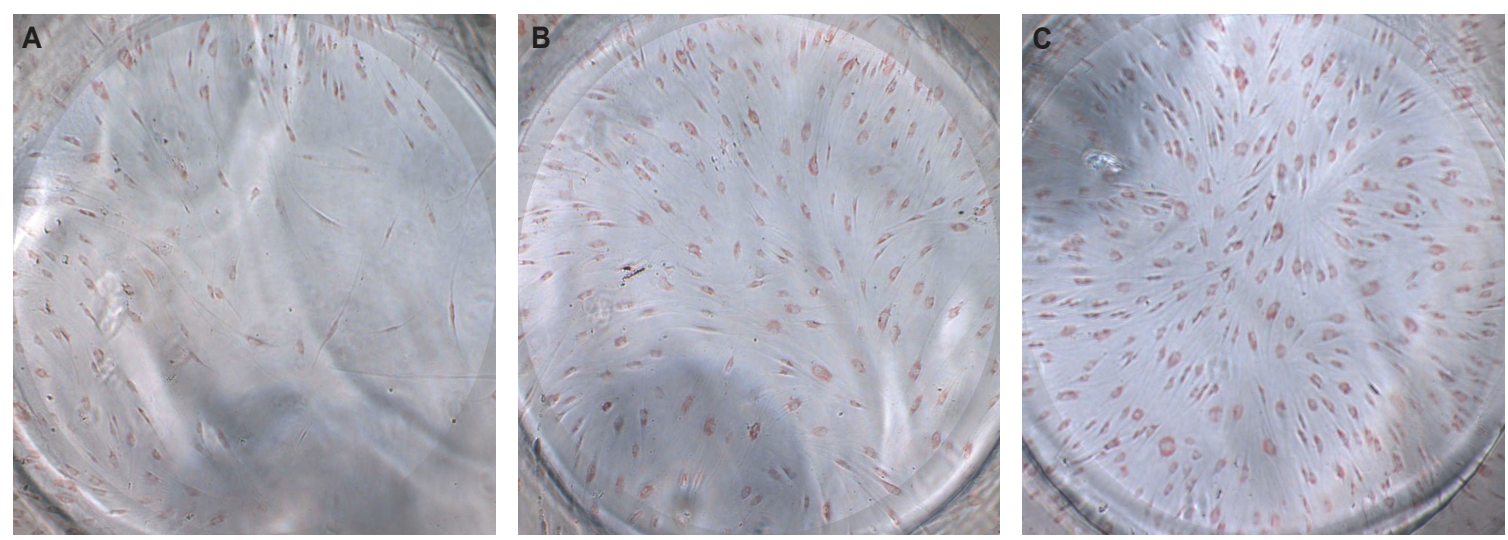

2 Figure 2: Agarose plug migration assay. After 48 hours MSCs responded to an increasing concentration of PDGF as shown above by representative images of agarose plugs containing

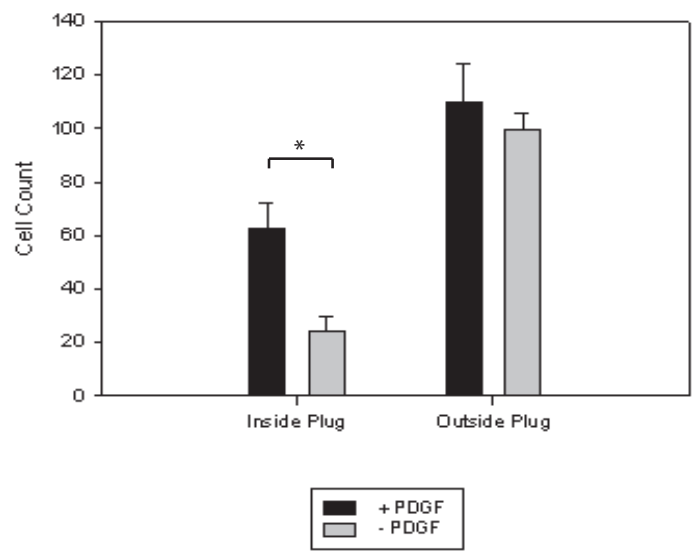

5

6 Figure 3: Comparison of migration and cell proliferation in agarose plug assay. The

7 increase in the number of cells shown inside the agarose central well is the result of migration

8 towards a higher concentration of PDGF and not an increase in cell proliferation in the whole

9 well. This is shown by the relative change in cell number within the central well induced by

$1010 \mathrm{ng} / \mathrm{mL}$ of PDGF; compared with no change in an area of the same size outside the well. *

11 Indicates a significant change compared with media control, using t-test $(P<0.05)$.

A gradient based chemotaxis assay for cell motility 
Plug assay
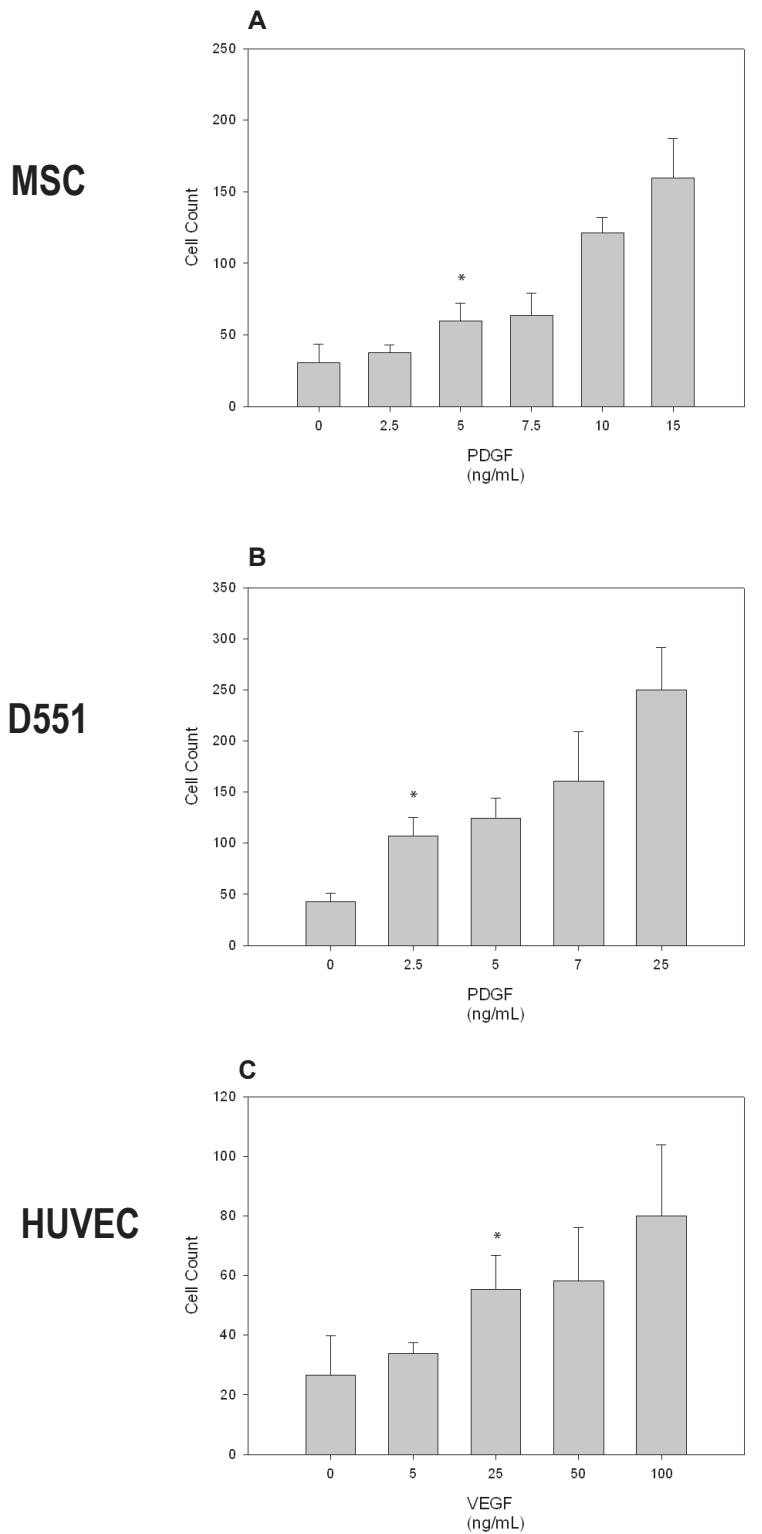

Scratch assay
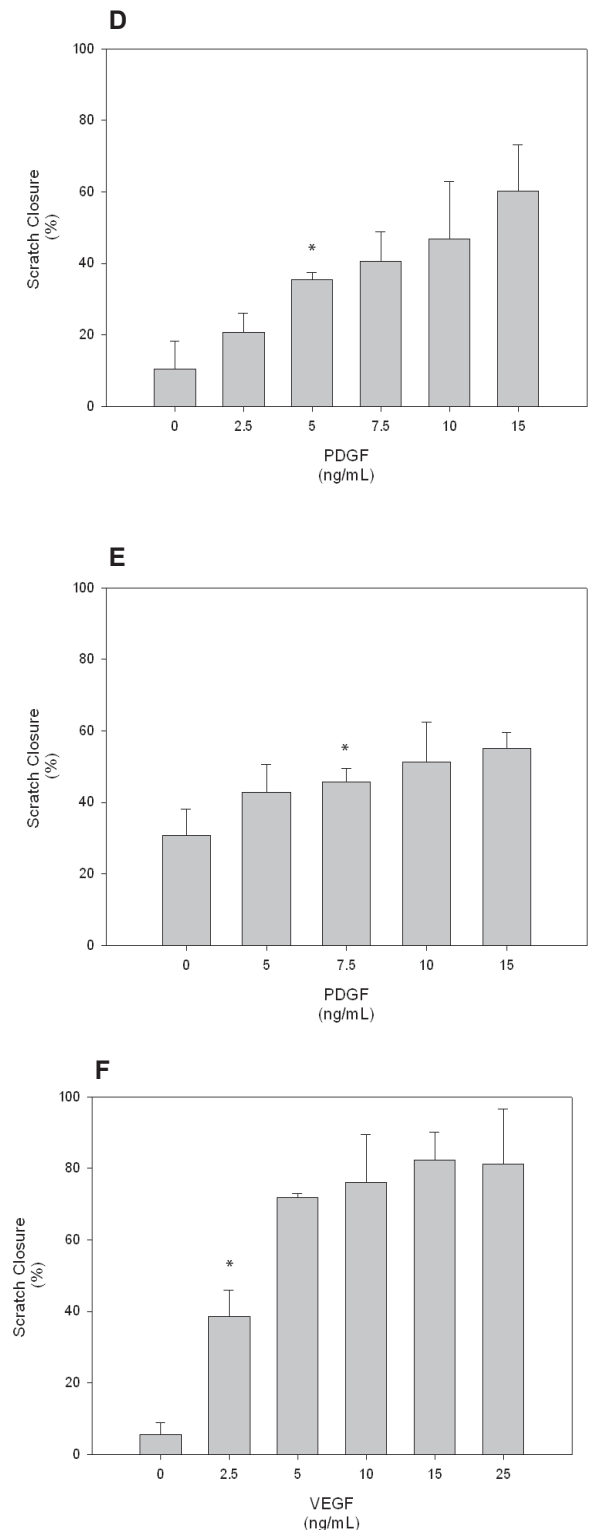

2 Figure 4: Comparison of agarose plug migration assay and scratch test. For all cell types

3 tested, the chemotactic agent induced a dose dependent change in the level of cell migration

4 as measured by a cell count in the central well of the agarose plug (A-C) and the percent

5 scratch closure in the scratch assay (D-F). MSCs (A and D) and D551 fibroblasts (B and E)

6 reponded to increasing concentrations of PDGF, while HUVECs ( $\mathbf{C}$ and $\mathbf{F})$ responded to 
1 VEGF. *Indicates the lowest concentration where a significant change is seen compared with

2 media control $(P<0.05)$.

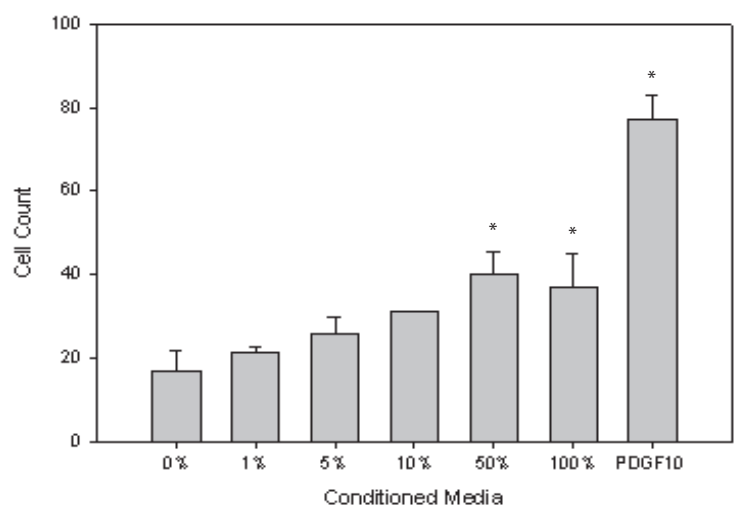

3 Figure 5: Migration of MSCs towards a gradient of $3 \mathrm{~T} 3$ cell conditioned media using

4 the agarose plug assay. The number of cells counted inside the central well of the agarose

5 plug depended on the concentration of cell conditioned media (diluted in normal media $\mathrm{v} / \mathrm{v}$ )

6 or presence of $10 \mathrm{ng} / \mathrm{mL}$ PDGF.

A gradient based chemotaxis assay for cell motility 